\title{
Data for secondary-electron production from ion precipitation at Jupiter II: Simultaneous and non-simultaneous target and projectile processes in collisions of $\mathrm{O}^{q+}+\mathrm{H}_{2}(q=0-8)$
}

\author{
D.R. Schultz ${ }^{\text {a,b,* }}$, H. Gharibnejad ${ }^{\text {c,b }}$, T.E. Cravens ${ }^{d}$, S.J. Houston ${ }^{d}$ \\ a Department of Physics and Astronomy, Northern Arizona University, Flagstaff, AZ 86011, United States \\ b Department of Physics, University of North Texas, Denton, TX 76203, United States \\ c National Institute of Standards and Technology, Gaithersburg, MD 20899, United States \\ d Department of Physics and Astronomy, University of Kansas, Lawrence, KS, 66045, United States
}

\section{A R T I C L E I N F O}

\section{Article history:}

Received 12 August 2018

Received in revised form 26 August 2018

Accepted 27 August 2018

Available online 11 October 2018

\begin{abstract}
A B S T R A C T
To improve the physical completeness of the data previously calculated (Schultz et al., 2017) to enable modeling of the effects of secondary electrons produced by energetic ion precipitation at Jupiter, we extend the treatment to include inelastic processes that occur simultaneously on the projectile $\left(\mathrm{O}^{q+}\right.$, $q=0-8)$ ) and target $\left(\mathrm{H}_{2}\right)$. Here, processes considered in the previous work (single and double ionization, transfer ionization, double capture with subsequent autoionization, single and double stripping, single and double charge transfer, and target excitation) reflecting non-simultaneous projectile and target electron transitions, are replaced with processes that include both non-simultaneous and simultaneous electronic transitions on the target and projectile. These include, for example, single ionization, single ionization with simultaneous single projectile excitation, single ionization with double projectile excitation, single ionization with single projectile stripping, and single ionization with double projectile stripping. Using this expanded set of processes, we show, via Monte Carlo ion-transport simulation, that improved representation of the energy deposition, measured by the stopping power, is obtained as compared to accepted recommended values for intermediate energies $(100-2000 \mathrm{keV} / \mathrm{u})$ where the stopping power is largest, while maintaining the existing good agreement with these recommended values for low $(\sim 10-$ $100 \mathrm{keV} / \mathrm{u})$ and high $(\geq 2000 \mathrm{keV} / \mathrm{u})$ energies. In addition, the ion-fraction distribution is altered by use of the improved data set. Both of these effects have implications for the energy deposition by oxygen ion precipitation in an $\mathrm{H}_{2}$ atmosphere. Therefore, use of this expanded data set can provide a more physically realistic secondary-electron distribution, and consequently improved atmospheric reaction network, improved description of ion contribution to atmospheric currents, and therefore improved understanding of Jovian ionosphere-atmosphere coupling.
\end{abstract}

(C) 2018 Elsevier Inc. All rights reserved.

\footnotetext{
* Corresponding author at: Department of Physics and Astronomy, Northern Arizona University, Flagstaff, AZ 86011, United States.

E-mail address: david.schultz@nau.edu (D.R. Schultz).
} 


\section{Contents}

1. Introduction 3



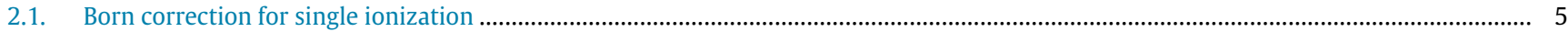

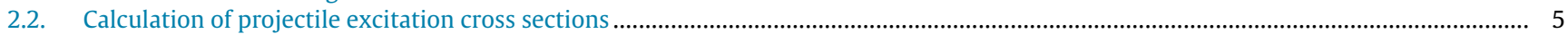

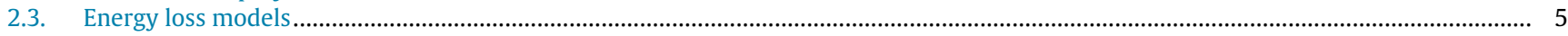

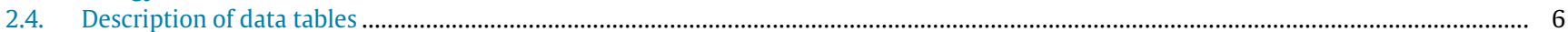

3. Simulation results: Stopping power, charge state fractions, and equilibrium charge state .................................................................................. 7

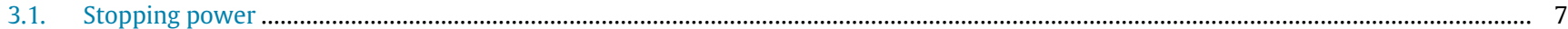

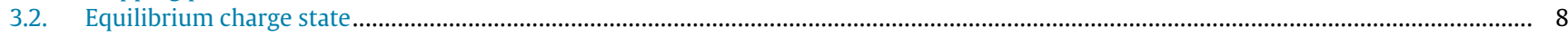



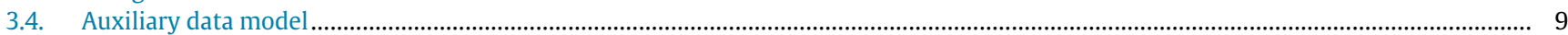

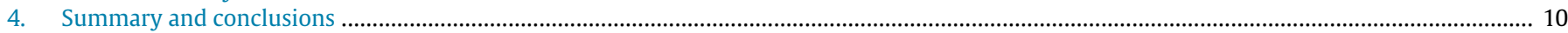

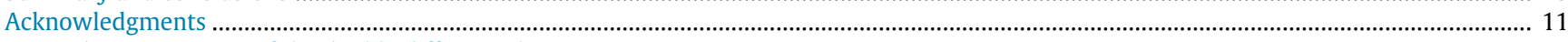

Appendix A. Survey of the doubly differential cross section .......................................................................................................................

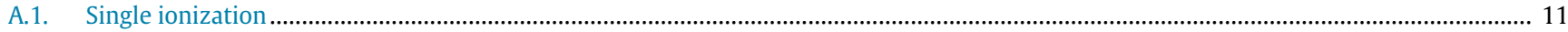





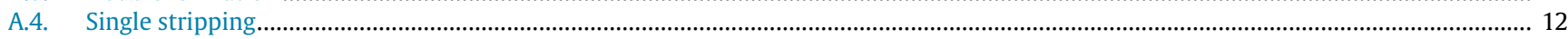

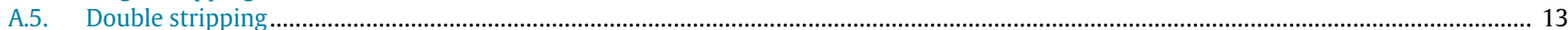

Appendix B. $\quad$ Transformation of ejected electron energies from the target to projectile frame ........................................................................ 13

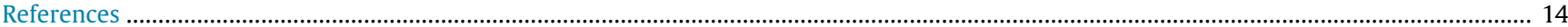

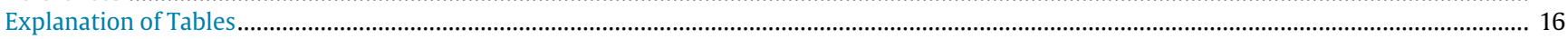

Table 1. The integral cross section and associated average energy loss for single and double stripping for $\mathrm{O}^{\mathrm{q}+}+\mathrm{H}_{2}(q=0-8)$ collisions with impact energies between 1 and $25000 \mathrm{keV} / \mathrm{u}$......................................................................................... 16

Table 2. The integral cross section and associated average energy loss for single and double projectile excitation for $\mathrm{O}^{\mathrm{q}+}+\mathrm{H}_{2}$

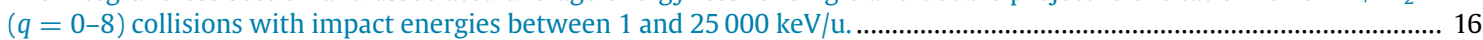

Table 3. The integral cross section and associated average energy loss for NSIM and SIM processes in $\mathrm{O}+\mathrm{H}_{2}$ collisions with

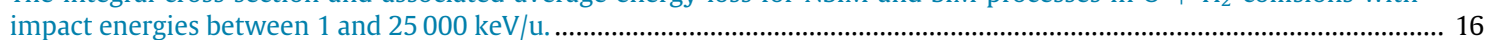

Table 4. The integral cross section and associated average energy loss for NSIM and SIM processes in $\mathrm{O}^{+}+\mathrm{H}_{2}$ collisions with

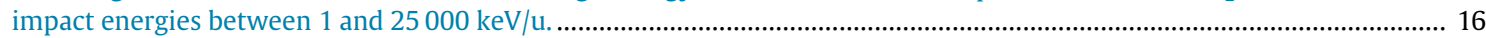

Table 5. The integral cross section and associated average energy loss for NSIM and SIM processes in $\mathrm{O}^{2+}+\mathrm{H}_{2}$ collisions with



Table 6. The integral cross section and associated average energy loss for NSIM and SIM processes in $\mathrm{O}^{3+}+\mathrm{H}_{2}$ collisions with impact energies between 1 and $25000 \mathrm{keV} / \mathrm{u}$................................................................................................................ 16

Table 7. The integral cross section and associated average energy loss for NSIM and SIM processes in $\mathrm{O}^{4+}+\mathrm{H}_{2}$ collisions with

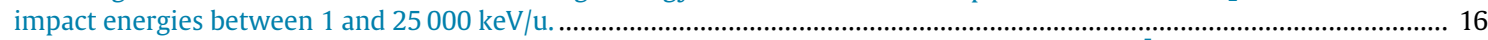

Table 8. The integral cross section and associated average energy loss for NSIM and SIM processes in $\mathrm{O}^{5+}+\mathrm{H}_{2}$ collisions with

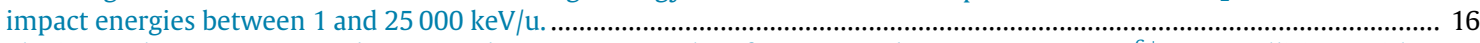

Table 9. The integral cross section and associated average energy loss for NSIM and SIM processes in $\mathrm{O}^{6+}+\mathrm{H}_{2}$ collisions with

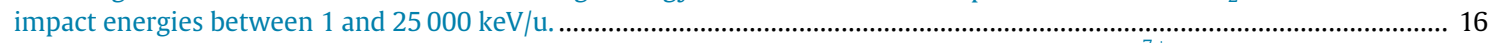

Table 10. The integral cross section and associated average energy loss for NSIM and SIM processes in $\mathrm{O}^{7+}+\mathrm{H}_{2}$ collisions with

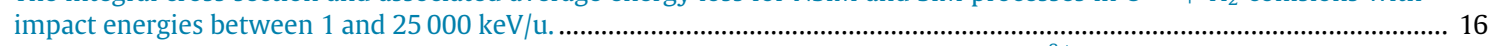

Table 11. The integral cross section and associated average energy loss for NSIM processes in $\mathrm{O}^{8+}+\mathrm{H}_{2}$ collisions with impact

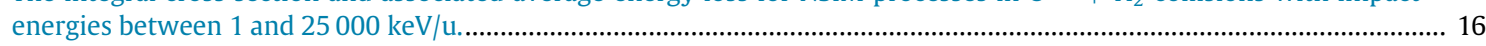

Table 12-14. The singly differential cross section as a function of the ejected electron energy for single ionization in 5000 -

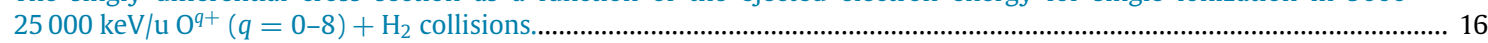

Table 15-17. The singly differential cross section as a function of the ejected electron angle for single ionization in $5000-25000 \mathrm{keV} / \mathrm{u}$



Table 18-20. The singly differential cross section as a function of the ejected electron energy for double ionization in $5000-$

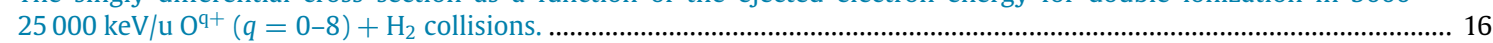

Table 21-23. The singly differential cross section as a function of the ejected electron angle for double ionization in $5000-25000 \mathrm{keV} / \mathrm{u}$

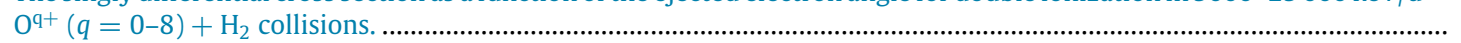

Table 24-26. The singly differential cross section as a function of the ejected electron energy for transfer ionization in $5000-$ $25000 \mathrm{keV} / \mathrm{u} \mathrm{O}^{\mathrm{q}+}(q=0-8)+\mathrm{H}_{2}$ collisions. ................................................................................................................ 16

Table 27-29. The singly differential cross section as a function of the ejected electron angle for transfer ionization in 5000$25000 \mathrm{keV} / \mathrm{u} \mathrm{O}^{\mathrm{q}+}(q=0-8)+\mathrm{H}_{2}$ collisions. ........................................................................................................... 16

Table 30-32. The singly differential cross section as a function of the ejected electron energy for single stripping in $5000-25000 \mathrm{keV} / \mathrm{u}$

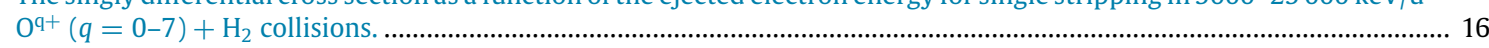

Table 33-35. The singly differential cross section as a function of the ejected electron angle for single stripping in $5000-25000 \mathrm{keV} / \mathrm{u}$ $\mathrm{Oq}+(q=0-7)+\mathrm{H}_{2}$ collisions

Table 36-38. The singly differential cross section as a function of the ejected electron energy for double stripping in 5000-

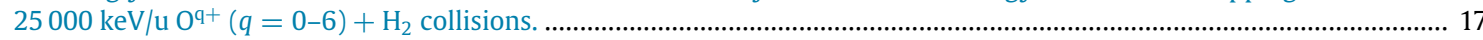

Table 39-41. The singly differential cross section as a function of the ejected electron angle for double stripping in $5000-25000 \mathrm{keV} / \mathrm{u}$ $\mathrm{O}^{\mathrm{q}+}(q=0-6)+\mathrm{H}_{2}$ collisions. 


\section{Introduction}

As summarized in our previous work [1] the need for data describing secondary-electron production in $1-2000 \mathrm{keV} / \mathrm{u} \mathrm{O}^{q+}$ $+\mathrm{H}_{2}(q=0-8)$ collisions is motivated by requirements for modeling dissociation of atmospheric molecules and contribution to currents arising from precipitation of these and other ions into the upper atmosphere of Jupiter [2,3]. Such data and their incorporation into ion-precipitation models was particularly timely in light of the arrival of the NASA Juno probe at Jupiter in July 2016 with the unique orbital characteristics to enable observations of the precipitating ion populations and their interactions with the upper atmosphere. The claim that such ionospheric ion populations contribute to atmospheric dynamics was bolstered by observations of polar auroral X-ray emissions subsequently identified [4-13] as originating from de-excitation emission following charge transfer between the precipitating ions and molecules of the upper atmosphere. In particular, this identification highlights the fact that $\mathrm{MeV} / \mathrm{u}$ oxygen and sulfur ions slow down in their passage through the atmosphere, producing secondary electrons, heating the atmospheric molecules, leading to dissociation of $\mathrm{H}_{2}$, and contributing the atmospheric currents, linking the Jovian ionosphere and atmosphere.

Therefore, a comprehensive data set of integral cross sections, which provides the probability of the various secondaryelectron-producing collisions or other energy- or charge-changing reactions, and the differential cross sections for secondaryelectron-production processes, which describe the energy and angle of ejection of the secondary electrons, was produced [1] using the best theoretical methods available in the general lack of measurements for the huge range of data required. Specifically, these processes included the following secondary-electron-producing reactions:

$$
\begin{aligned}
& \mathrm{O}^{q+}+\mathrm{H}_{2} \rightarrow \mathrm{O}^{q+}+\mathrm{H}_{2}^{+}+\mathrm{e} ; \mathrm{O}^{q+}+ \\
& \mathrm{H}+\mathrm{H}^{+}+\mathrm{e} \\
& \mathrm{O}^{q+}+\mathrm{H}_{2} \rightarrow \mathrm{O}^{(q-1)+}+\mathrm{H}^{+}+\mathrm{H}^{+}+\mathrm{e} \\
& \mathrm{O}^{q+}+\mathrm{H}_{2} \rightarrow \mathrm{O}^{q+}+\mathrm{H}^{+}+\mathrm{H}^{+}+\mathrm{e}+\mathrm{e} \\
& \mathrm{O}^{q+}+\mathrm{H}_{2} \rightarrow \mathrm{O}^{(q-2)+}+\mathrm{H}^{+}+\mathrm{H}^{+} \\
& \quad \rightarrow \mathrm{O}^{(q-1)+}+\mathrm{e} \\
& \mathrm{O}^{q+}+\mathrm{H}_{2} \rightarrow \mathrm{O}^{(q+1)+}+\mathrm{X}+\mathrm{e} \\
& \mathrm{O}^{q+}+\mathrm{H}_{2} \rightarrow \mathrm{O}^{(q+2)+}+\mathrm{X}+\mathrm{e}+\mathrm{e}
\end{aligned}
$$

single ionization (SI)
transfer ionization (TI)
double ionization (DI)
double-capture autoionization (DCAI)
single stripping (SS)
double stripping (DS)

where $q$ runs from 0 to 8 , depending on the number of electrons possibly involved (e.g., for $\mathrm{O}^{+} \mathrm{DCAI}$ is not considered, or for $\mathrm{O}^{7+}$ DS is not possible) and where $\mathrm{X}$ stands for any product (i.e., $\mathrm{H}_{2}$, $\mathrm{H}+\mathrm{H}, \mathrm{H}_{2}^{*}$ ). Data was also needed for the processes that do not produce secondary electrons but that change the charge of the ion or contribute significantly to the energy loss experienced by the ion as it slows down through collisions. These are

$$
\begin{array}{ll}
\mathrm{O}^{q+}+\mathrm{H}_{2} \rightarrow \mathrm{O}^{(q-1)+}+\mathrm{H}_{2}^{+} ; \mathrm{O}^{(q-1)+}+\mathrm{H}+\mathrm{H}^{+} & \text {single electron capture (SC) } \\
\mathrm{O}^{q+}+\mathrm{H}_{2} \rightarrow \mathrm{O}^{(q-2)+}+\mathrm{H}^{+}+\mathrm{H}^{+} & \text {double electron capture (DC) } \\
\mathrm{O}^{q+}+\mathrm{H}_{2} \rightarrow \mathrm{O}^{q+}+\mathrm{H}_{2}^{*} & \text { gross target excitation (TEX) }
\end{array}
$$

with similar restriction on $q$ depending on the number of electrons on the projectile. Collision energies were considered from 2000 $\mathrm{keV} / \mathrm{u}$, corresponding to the upper limit for the energy previously estimated (see Refs. [2-5]) for the initial energy at the top of the atmosphere, to $1 \mathrm{keV} / \mathrm{u}$, at which point the secondary-electron production drops off rapidly.

As noted [1], very little data from measurements exists for comparison with this very large, computationally generated data set. Previous work considered comparisons with the limited experimental data available and also drew on assessment of the accuracy of results in the context of numerous similar comparisons for other collision systems for which more experimental data is available. In addition, the data set was tested by computing, using a Monte
Carlo ion-trajectory simulation, the stopping power. This quantity gives a measure of the energy deposition in the transport of ions through the gaseous medium taking into account the totality of the processes occurring for a given impact energy, as opposed to testing the integral or differential cross sections for a single charge state and collision energy. This simulation has the property that it closely parallels the actual ion-precipitation models used to study the transport of the ions and the secondary-electron production in Jupiter's atmosphere [2,3].

While very good agreement with recommended values of the stopping power was found at both low collision energy (10$100 \mathrm{keV} / \mathrm{u})$ and high collision energy ( $2000 \mathrm{keV} / \mathrm{u})$, regimes in which experimental measurements of the stopping power directly constrain the recommended values, our simulation results underestimated the recommended stopping power by up to a factor of almost two in the intermediate energy regime (100-2000 keV/u). Having assessed the accuracy of the integral and differential cross sections as well as possible, the conclusion was that the agreement was the best that could be obtained given the present treatment of the collision processes and the uncertainty indicated by the variation between the 2000 and 2013 recommended stopping power.

Subsequent work has revealed the origin of this discrepancy. At intermediate collision energies, modeling using the set of processes listed above has missing energy loss processes, inclusion of which results in significantly better agreement with the recommended stopping power. The improved agreement indicates that better representation of the energy deposition by oxygen ions precipitating into the upper atmosphere of Jupiter and improved description of the secondary-electron spectrum would be obtained using an expanded reaction network. In particular, we present here data considering both non-simultaneous and simultaneous projectile and target processes that yield this improved agreement with the recommended stopping power. That is, in previous work only processes occurring on the target or projectile independently were considered, whereas here we take into account processes in which an electronic transition (e.g., excitation or ionization) can take place on both the target and projectile at the same time. As described below, this expanded set of processes not only improves agreement of the calculated stopping power at intermediate energies, but it also preserves the good agreement previously obtained at low and high energies.

While an expanded set of integral cross sections is required to treat both non-simultaneous and simultaneous projectile and target processes, the same set of differential cross sections for the electron ejection angle and energy calculated in the previous work [1] may be used without modification. Specifically, to simulate the secondary-electron production in a collision event, instead of selecting an electron angle and energy for a single process such as SI, one uses combinations of samplings for the simultaneous processes such as SI with simultaneous SS for example.

The non-simultaneous and simultaneous projectile and target processes included in the expanded set are the following:

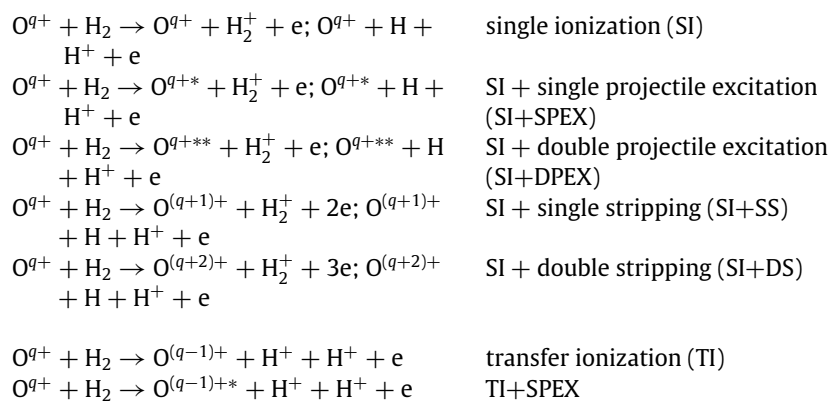




\begin{tabular}{|c|c|}
\hline $\begin{array}{l}\mathrm{O}^{q+}+\mathrm{H}_{2} \rightarrow \mathrm{O}^{(q-1)+* *}+\mathrm{H}^{+}+\mathrm{H}^{+}+\mathrm{e} \\
\mathrm{O}^{q+}+\mathrm{H}_{2} \rightarrow \mathrm{O}^{q+}+\mathrm{H}^{+}+\mathrm{H}^{+}+2 \mathrm{e} \\
\mathrm{O}^{q+}+\mathrm{H}_{2} \rightarrow \mathrm{O}^{(q+1)+}+\mathrm{H}^{+}+\mathrm{H}^{+}+3 \mathrm{e}\end{array}$ & $\begin{array}{l}\text { TI+DPEX } \\
\text { TI+SS } \\
\text { TI+DS }\end{array}$ \\
\hline $\begin{array}{l}\mathrm{O}^{q+}+\mathrm{H}_{2} \rightarrow \mathrm{O}^{q+}+\mathrm{H}^{+}+\mathrm{H}^{+}+2 \mathrm{e} \\
\mathrm{O}^{q+}+\mathrm{H}_{2} \rightarrow \mathrm{O}^{q+*}+\mathrm{H}^{+}+\mathrm{H}^{+}+2 \mathrm{e} \\
\mathrm{O}^{q+}+\mathrm{H}_{2} \rightarrow \mathrm{O}^{q+* *}+\mathrm{H}^{+}+\mathrm{H}^{+}+2 \mathrm{e} \\
\mathrm{O}^{q+}+\mathrm{H}_{2} \rightarrow \mathrm{O}^{(q+1)+}+\mathrm{H}^{+}+\mathrm{H}^{+}+3 \mathrm{e} \\
\mathrm{O}^{q+}+\mathrm{H}_{2} \rightarrow \mathrm{O}^{(q+2)+}+\mathrm{H}^{+}+\mathrm{H}^{+}+4 \mathrm{e}\end{array}$ & $\begin{array}{l}\text { double ionization (DI) } \\
\text { DI+SPEX } \\
\text { DI+DPEX } \\
\text { DI+SS } \\
\text { DI+DS }\end{array}$ \\
\hline $\begin{array}{l}\mathrm{O}^{q+}+\mathrm{H}_{2} \rightarrow \mathrm{O}^{(q-2)+* *}+\mathrm{H}^{+}+\mathrm{H}^{+} \rightarrow \\
\quad \mathrm{O}^{(q-1)+}+\mathrm{e}\end{array}$ & $\begin{array}{l}\text { double-capture autoionization } \\
\text { (DCAI) }\end{array}$ \\
\hline $\begin{array}{l}\mathrm{O}^{q+}+\mathrm{H}_{2} \rightarrow \mathrm{O}^{(q-2)+* * *}+\mathrm{H}^{+}+\mathrm{H}^{+} \rightarrow \\
\quad \mathrm{O}^{(q-1)+*}+\mathrm{e}\end{array}$ & DCAI+SPEX \\
\hline $\begin{array}{l}\mathrm{O}^{q+}+\underset{\mathrm{O}^{(q-1)+* *}}{\mathrm{H}_{2}} \rightarrow \mathrm{O}^{(q-2)+* * * *}+\mathrm{H}^{+}+\mathrm{H}^{+} \rightarrow \\
\end{array}$ & $\mathrm{DCAI}+\mathrm{DPEX}$ \\
\hline $\begin{array}{l}\mathrm{O}^{q+}+\mathrm{H}_{2} \rightarrow \mathrm{O}^{(q-2)+* *}+\mathrm{H}^{+}+\mathrm{H}^{+} \rightarrow \\
\mathrm{O}^{q+}+2 \mathrm{e}\end{array}$ & $\mathrm{DCAI}+\mathrm{SS}$ \\
\hline $\begin{array}{c}\mathrm{O}^{q+}+\mathrm{H}_{2} \rightarrow \mathrm{O}^{(q-2)+* *}+\mathrm{H}^{+}+\mathrm{H}^{+} \rightarrow \\
\quad \mathrm{O}^{(q+1)+}+3 \mathrm{e}\end{array}$ & $\mathrm{DCAI}+\mathrm{DS}$ \\
\hline $\begin{array}{l}\mathrm{O}^{q+}+\mathrm{H}_{2} \rightarrow \mathrm{O}^{(q-1)+}+\mathrm{H}_{2}^{+} ; \mathrm{O}^{(q-1)+}+\mathrm{H} \\
+\mathrm{H}^{+}\end{array}$ & single electron capture (SC) \\
\hline $\begin{array}{l}\mathrm{O}^{q+}+\mathrm{H}_{2} \rightarrow \mathrm{O}^{(q-1)+*}+\mathrm{H}_{2}^{+} ; \mathrm{O}^{(q-1)+*}+ \\
\mathrm{H}+\mathrm{H}^{+}\end{array}$ & SC+SPEX \\
\hline $\begin{array}{l}\mathrm{O}^{q+}+\mathrm{H}_{2} \rightarrow \mathrm{O}^{(q-1)+* *}+\mathrm{H}_{2}^{+} ; \mathrm{O}^{(q-1)+* *} \\
+\mathrm{H}+\mathrm{H}^{+}\end{array}$ & SC+DPEX \\
\hline $\begin{array}{l}\mathrm{O}^{q+}+\mathrm{H}_{2} \rightarrow \mathrm{O}^{(q)+}+\mathrm{H}_{2}^{+}+\mathrm{e} ; \mathrm{O}^{(q)+}+\mathrm{H} \\
+\mathrm{H}^{+}+\mathrm{e}\end{array}$ & $\mathrm{SC}+\mathrm{SS}$ \\
\hline $\begin{aligned} \mathrm{O}^{q+} & +\mathrm{H}_{2} \rightarrow \mathrm{O}^{(q+1)+}+\mathrm{H}_{2}^{+}+2 \mathrm{e} ; \mathrm{O}^{(q+1)+} \\
& +\mathrm{H}+\mathrm{H}^{+}+2 \mathrm{e}\end{aligned}$ & $\mathrm{SC}+\mathrm{DS}$ \\
\hline $\mathrm{O}^{q+}+\mathrm{H}_{2} \rightarrow \mathrm{O}^{(q-2)+}+\mathrm{H}^{+}+\mathrm{H}^{+}$ & double electron capture (DC) \\
\hline $\mathrm{O}^{q+}+\mathrm{H}_{2} \rightarrow \mathrm{O}^{(q-2)+*}+\mathrm{H}^{+}+\mathrm{H}^{+}$ & $\mathrm{DC}+$ SPEX \\
\hline $\mathrm{O}^{q+}+\mathrm{H}_{2} \rightarrow \mathrm{O}^{(q-2)+* *}+\mathrm{H}^{+}+\mathrm{H}^{+}$ & DC+DPEX \\
\hline $\mathrm{O}^{q+}+\mathrm{H}_{2} \rightarrow \mathrm{O}^{(q-1)+}+\mathrm{H}^{+}+\mathrm{H}^{+}+\mathrm{e}$ & $\mathrm{DC}+\mathrm{SS}$ \\
\hline $\mathrm{O}^{q+}+\mathrm{H}_{2} \rightarrow \mathrm{O}^{(q)+}+\mathrm{H}^{+}+\mathrm{H}^{+}+2 \mathrm{e}$ & $\mathrm{DC}+\mathrm{DS}$ \\
\hline $\mathrm{O}^{q+}+\mathrm{H}_{2} \rightarrow \mathrm{O}^{q+}+\mathrm{H}_{2}^{*}$ & target excitation (TEX) \\
\hline $\mathrm{O}^{q+}+\mathrm{H}_{2} \rightarrow \mathrm{O}^{q+*}+\mathrm{H}_{2}^{*}$ & TEX+SPEX \\
\hline $\mathrm{O}^{q+}+\mathrm{H}_{2} \rightarrow \mathrm{O}^{q+* *}+\mathrm{H}_{2}^{*}$ & TEX+DPEX \\
\hline $\mathrm{O}^{q+}+\mathrm{H}_{2} \rightarrow \mathrm{O}^{(q+1)+}+\mathrm{H}_{2}^{*}+\mathrm{e}$ & $\mathrm{TEX}+\mathrm{SS}$ \\
\hline $\mathrm{O}^{q+}+\mathrm{H}_{2} \rightarrow \mathrm{O}^{(q+2)+}+\mathrm{H}_{2}^{*}+2 \mathrm{e}$ & TEX+DS \\
\hline
\end{tabular}

In what follows, we describe the model and new calculations by which the integral cross sections for these processes have been determined (Section 2), describe the tabulations of the new data (Section 2), and demonstrate the good agreement obtained over the full energy range considered between the stopping power derived from our Monte Carlo simulation using the new data set and the recommended stopping power (Section 3). Owing to the existence of measurements of the stopping power at energies higher than $2000 \mathrm{keV} / \mathrm{u}$ that can provide a further test of the data through ion-transport modeling, here we extend the calculations to $25000 \mathrm{keV} / \mathrm{u}$.

\section{Model for non-simultaneous and simultaneous projectile and target processes}

As described in the previous work [1], treating the full range of non-simultaneous (NSIM) target or projectile processes (SI, TI, DI, DCAI, SS, DS, SC, DC, and TEX) for all charges states $(q=0-8)$ of oxygen colliding with $\mathrm{H}_{2}$ over the energy range of $1-2000 \mathrm{keV} / \mathrm{u}$ is presently only achievable with the classical trajectory Monte Carlo (CTMC) method [14,15], and in particular with the CTMC models developed for $\mathrm{H}_{2}[16,17]$ (target processes) or for multi-electron atoms and ions (nCTMC) [18] (here used for projectile processes). Critical comparisons of the secondary-electron production using the CTMC method with measurements and other theoretical methods, noted in Ref. [1], have been made for proton [19] and $\mathrm{He}^{+}[20]$ impact of $\mathrm{H}$; fluorine ions colliding with $\mathrm{H}_{2}$ [21]; $\mathrm{C}^{+}$[22], $\mathrm{F}^{9+}$ [23], and highly charged gold ion [24] impact of $\mathrm{He}$; and carbon ion impact of neon [25], for example, along with the new comparisons made in that work.

In the treatment of target processes, electrons on the projectile were considered inactive, their effect being to provide an internuclear-distance-dependent screening of the projectile nuclear charge via a model potential (see, e.g., Ref. [26] describing the model potential and its use for oxygen ion impact of atomic hydrogen). Similarly, to treat projectile processes, the electrons of the target were considered inactive and the multi-electron projectile was described by the nCTMC model in which the electrons are bound with either the ion/atom's sequential binding energies or with equal binding energies but ignoring the electron-electron interaction except via a screened Coulomb potential.

To treat simultaneous (SIM) processes on the projectile and target in an analogous way would require active electrons on both centers and inclusion of the electron-electron interaction. However, the electron-electron interaction destabilizes the classical model of the atom/molecule rendering results of such a direct generalization of the method of limited utility. We note that in previous work [27] we implemented a CTMC model including active electrons on both the target and projectile, obtaining very good agreement with experimental results for the coincident charge state distributions in collisions of $\mathrm{F}^{6+}$ with $\mathrm{Ne}$, including only projectile-electron-target-electron interactions. That work showed significant improvement over the independent electron model, but treated only a single electron on the projectile and no electron-electron interaction within the projectile or target. We are not aware of any subsequent attempts to extend such a model to more active and interacting electrons.

Therefore, a method by which SIM processes can be treated using independently calculated input for target and projectile processes is necessary. The essence of the model that we have developed is to partition the integral cross sections for the NSIM processes among the possible SIM processes. That is, the CTMC calculation leading to an NSIM process, say SI, is achieved without knowing the fraction of collision events in which a simultaneous process occurs that changes the projectile energy (ion slowing) or charge (e.g., stripping). We therefore partition the NSIM integral cross section for SI into fractions for SI occurring without any simultaneous projectile process, SI occurring simultaneously with projectile single excitation (SI+SPEX), with double excitation (SI+DPEX), with single stripping (SI+SS), and with double stripping (SI+DS).

The fractions are determined by the overlap of the probability as a function of impact parameter, $P(b)$, for the projectile process with that for the target process. Since the integral of $P(b)$ gives the cross section, or area, corresponding to the reaction, that is

$\sigma=\int_{0}^{b_{\max }} P(b) b d b$

this fraction reflects the relative probability of the projectile and target processes. For the vast majority of the oxygen charge states and impact energies, the target processes are more probable and have larger $b_{\max }$, owing to the low charge state of the target nuclei and low ionization potential of $\mathrm{H}_{2}$, so that the maximum impact parameter for the projectile processes is significantly smaller than that for the target processes. Therefore, the projectile-process fractions are generally much smaller than one, and consequently, the calculated integral cross section for the target processes can be "divided up" into the possible SIM processes.

Further, since the target and projectile processes are all calculated separately, the method must preserve unitarity of probability so that the sum of all the SIM processes adds up to that which is computed for the corresponding NSIM process. To illustrate this, consider again the example of the partition of the SI process into the possible SIM processes, SI+SPEX, SI+DPEX, SI+SS, 
and SI+DS. Then the corresponding SIM-process cross sections, $\sigma_{S I}(\mathrm{SIM}), \sigma_{S I+S P E X}(\mathrm{SIM}), \sigma_{S I+D P E X}(\mathrm{SIM}), \sigma_{S I+S S}(\mathrm{SIM})$, and $\sigma_{S I+D S}(\mathrm{SIM})$, must sum to the calculated NSIM-process cross section $\sigma_{S I}$ (NSIM).

This may be accomplished as follows. The CTMC calculations yield the NSIM-process fractions $f_{\text {SPEX }}, f_{\text {DPEX }}, f_{\text {SS }}$, and $f_{\text {DS }}$. First, we determine which of the SIM processes has the largest fraction, $f_{\max }$. This is frequently SI+SPEX, for example, so in that case, $f_{\max }=f_{\text {SPEX }}$. This sets how much of the non-simultaneous process remains, which in this example yields $f_{\mathrm{SI}}=1-f_{\max }$, with the corresponding integral cross section $\sigma_{\mathrm{SI}}(\mathrm{SIM})=\left(1-f_{\max }\right) \sigma_{\mathrm{SI}}(\mathrm{NSIM})$, where $\sigma_{\mathrm{SI}}(\mathrm{NSIM})$ is the integral cross section calculated considering only single ionization of the target, and $\sigma_{\mathrm{SI}}(\mathrm{SIM})$ is our model for the corresponding single ionization of the target considering loss of flux to the simultaneous projectile processes.

Next, we distribute the remaining probability among the other SIM processes by normalizing the fractions by $f_{\max }$, that is, by using the normalizing factor $F=\Sigma_{\alpha} f_{\alpha} / f_{\max }$, where $\alpha=$ SI+SPEX, SI+DPEX, SI+SS, or SI+DS. In the present example that is $\sigma_{\mathrm{SI}+\mathrm{SS}}(\mathrm{SIM})=f_{\mathrm{SS}} / F \quad \sigma_{\mathrm{SI}}(\mathrm{NSIM})$, etc. This process ensures that $\sigma_{\mathrm{SI}}(\mathrm{NSIM})=\sigma_{\mathrm{SI}}(\mathrm{SIM})+\sigma_{\mathrm{SI}+\mathrm{SPEX}}(\mathrm{SIM})+\sigma_{\mathrm{SI}+\mathrm{DPEX}}(\mathrm{SIM})+$ $\sigma_{\mathrm{SI}+\mathrm{SS}}(\mathrm{SIM})+\sigma_{\mathrm{SI}+\mathrm{DS}}(\mathrm{SIM})$.

To illustrate this process concretely, let us consider the SI in 500 $\mathrm{keV} / \mathrm{u} \mathrm{O}^{2+}+\mathrm{H}_{2}$ as treated in the present model. For each of the projectile processes, we use the CTMC model to compute $b_{\max }$, the impact parameter beyond which the process becomes negligible. We next determine $f_{\alpha}$ as the number of SI events occurring within the corresponding $b_{\max }$ divided by the total number of Monte Carlo trajectories in the calculation of the NSIM SI cross section. For the present example, we obtain

$f_{\mathrm{SS}}=0.513, f_{\mathrm{DS}}=0.261, f_{\mathrm{SPEX}}=0.565, f_{\mathrm{DPEX}}=0.308$

which yields $f_{\max }=0.565, \Sigma_{\alpha} f_{\alpha}=1.647, F=\Sigma_{\alpha} / f_{\max }=2.915$, and $\Sigma_{\alpha} f_{\alpha} / F=1$. From these, we then compute the five SIMprocess cross sections as follows:

$$
\begin{aligned}
\sigma_{\mathrm{SI}}(\mathrm{SIM}) & =\sigma_{\mathrm{SI}}(\mathrm{NSIM}) *\left(1-f_{\max }\right) \\
& =2.34 \times 10^{-16} * 0.435=1.02 \times 10^{-16} \\
\sigma_{\mathrm{SI}+\mathrm{SS}}(\mathrm{SIM}) & =\sigma_{\mathrm{SI}}(\mathrm{NSIM}) * f_{\mathrm{SS}} / F \\
& =2.34 \times 10^{-16} * 0.513 / 2.915=4.12 \times 10^{-17} \\
\sigma_{\mathrm{SI}+\mathrm{DS}}(\mathrm{SIM}) & =\sigma_{\mathrm{SI}}(\mathrm{NSIM}) * f_{\mathrm{DS}} / F \\
& =2.34 \times 10^{-16} * 0.261 / 2.915=2.10 \times 10^{-17} \\
\sigma_{\mathrm{SI}+\mathrm{SPEX}}(\mathrm{SIM}) & =\sigma_{\mathrm{SI}}(\mathrm{NSIM}) * f_{\mathrm{SPXX}} / F \\
& =2.34 \times 10^{-16} * 0.565 / 2.915=4.54 \times 10^{-17} \\
\sigma_{\mathrm{SI}+\mathrm{DPEX}}(\mathrm{SIM}) & =\sigma_{\mathrm{SI}}(\mathrm{NSIM}) * f_{\mathrm{DPEX}} / F \\
& =2.34 \times 10^{-16} * 0.308 / 2.915=2.47 \times 10^{-17}
\end{aligned}
$$

where all cross sections are given in units of $\mathrm{cm}^{2}$. Note that as required $\Sigma_{\alpha} \sigma_{\mathrm{SI}+\alpha}(\mathrm{SIM})=\sigma_{\mathrm{SI}}(\mathrm{NSIM})$. Also note that in this model, since the $b_{\max }$ for the projectile processes is smaller than that for the target processes, probability for these are "used up" in the SIM processes and therefore there is no stripping or projectile excitation that does not occur simultaneously with a target process.

\subsection{Born correction for single ionization}

As noted in the previous work [1], SI is the dominant secondaryelectron production mechanism for much of the impact energy range considered, and so an important correction to the CTMC results for both the integral and differential cross sections is the dominant quantum mechanical effect not present in the method. In particular, we described how we have added that portion of the cross section from the Born approximation that represents the missing effect, namely production of the lowest energy ejected electrons through dipole transitions that are classically suppressed $[19,28]$.

Here, since we partition the NSIM SI integral cross section into the SIM-processes integral cross sections, we must also partition the Born approximation correction. Since the portion of the Born approximation cross section that we need to add comes mostly from large impact parameters (from about $90 \%$ of the $b_{\max }$ found from the CTMC SI calculation to approximately twice this value [28], the precise upper value not effecting the result), we add the probability from the Born correction in this range to the probability from the CTMC calculation. With this corrected $P(b)$, we then apply the method described above to compute the partitions of the NSIM SI integral cross section into those for the SIM SI processes.

For the single differential cross sections, the same procedure as used in the previous work [1] is applied since there is no need to partition the NSIM differential cross sections as described below in the subsection on calculation of the projectile energy loss.

\subsection{Calculation of projectile excitation cross sections}

The previous treatment of the NSIM processes [1] included non-simultaneous stripping of the projectile, but careful investigation of what other processes occurring on the projectile could significantly contribute to the energy loss when SIM processes are considered indicated that projectile excitation should be included.

The new calculations, for SPEX and DPEX, proceed in a manner analogous to the TEX calculations described before [1]. In this case, the active electrons are on the projectile and a model potential is used to represent the impacting neutral hydrogen. Instead of attempting to create such a potential with the two nuclear centers of the hydrogen molecule, experience indicates that collisions close enough to produce excitation typical occur with one center or the other, and so the simplifying assumption of using an effective " $H$ " atom impacting the oxygen atom or ion, with mass given by that of $\mathrm{H}_{2}$, is used.

Specifically, for each oxygen ionization stage (i.e., $\mathrm{O}\left(1 \mathrm{~s}^{2} 2 \mathrm{~s}^{2} 2 \mathrm{p}^{4}\right)$, $\left.\mathrm{O}^{+}\left(1 \mathrm{~s}^{2} 2 \mathrm{~s}^{2} 2 \mathrm{p}^{3}\right), \ldots\right)$, the nCTMC method is employed. For SPEX, the electrons in an active shell (i.e, for $\mathrm{O}$, the four least bound electrons) are included all with the same binding energy, since the single electron transition affects dominantly one of them at a time and including all four properly represents the increased probability from having that many equivalent electrons. For DPEX, the electrons in the active shell are given the experimentally determined sequential ionization potentials, because proper modeling of the two-electron transitions requires the correct energy deposition.

As for TEX, SPEX is judged to have occurred when one of the active electrons is excited above the ground state but remaining below the ionization potential. For DPEX, two electrons must be excited above the ground states and below their ionization potentials. The number of events in the CTMC calculation for single projectile excitation, $N_{\text {SPEX }}$, yields the integral cross section

$\sigma_{\mathrm{SPEX}}=\pi a_{o}^{2} b_{\max }^{2} \frac{N_{\mathrm{SPEX}}}{N_{\mathrm{MC}}}$

where $a_{o}$ is the Bohr radius $\left(0.529 \times 10^{-8} \mathrm{~cm}\right), b_{\max }$ is the maximum impact parameter in the CTMC simulation for which SPEX occurs, and $N_{\mathrm{MC}}$ is the number of Monte Carlo events in the CTMC simulation. An analogous expression gives the integral cross section for DPEX.

\subsection{Energy loss models}

Accompanying each of the NSIM or SIM processes is an associated energy loss. The energy loss reflects the inelasticity of the collision in each channel and slows the projectile during its passage 
through the target medium. Most of the energy loss models given in the previous work describing the NSIM processes are directly applicable since for the SIM processes one simply adds the target and projectile energy losses.

For example, the previous work described the energy loss model for SI as consisting of the amount of energy needed to singly ionize the target molecule (ionization potential, $\operatorname{IP}_{1}\left(\mathrm{H}_{2}\right)$ ) plus the amount of energy imparted to the ejected electron. The average energy loss, a quantity useful in computing the stopping power, for example, is therefore given as $\Delta \mathrm{e}^{\mathrm{SI}}=\mathrm{IP}_{1}\left(\mathrm{H}_{2}\right)+\left\langle\mathrm{E}_{e}^{\mathrm{SI}}(\mathrm{E})\right\rangle$, where $\left\langle\mathrm{E}_{e}^{\mathrm{SI}}(\mathrm{E})\right\rangle$ is the average energy of an electron ejected in single ionization at a given impact energy. Also, of even greater utility for transport simulations seeking to describe the ejected electron spectrum, instead of the average ejected electron energy one can sample the distribution of electron energies given by the singly differential cross section (SDCS) for SI at a given impact energy. Such a sampling on average will give the same energy loss as the expression above. Coupled with sampling of the ejected electron angle from the corresponding SDCS, a transport simulation can determine the distribution of secondary-electron-emission energies and angles.

Reflecting an even greater level of physical description of the ejected-electron process, the doubly differential cross section (DDCS) could be utilized. The DDCS reflects the correlation between ejected-electron energy and angle, as surveyed in Appendix A for the presently considered collisions and secondaryelectron-producing reactions. However, to implement its use would add greater complexity to transport simulations and would require significantly larger CTMC calculations to generate enough Monte Carlo ejected-electron events to bin them with good statistics into not just two, one-dimensional arrays (i.e., the SDCS as a function of ejected-electron energy and the SDCS as a function of the ejected-electron angle), but in one, two-dimensional array (i.e, the DDCS as a simultaneous function of ejected-electron energy and angle).

For the SIM processes, the average energy loss is given simply as the sum of the average energy loss for the target channel and the average energy loss for the projectile channel. That is, when both a target and projectile process occurs, one adds the energy losses from each. In that case as well, a transport simulation can use either the average energy loss computed in this way or can sample the SDCSs for both the target and projectile channel that results in electron emission (e.g., SI+SS).

Table A summarizes the average energy loss models for each NSIM process, as described in Ref. [1], augmented here by expressions for SPEX and DPEX. For SPEX, the quantity $\left\langle\Delta \mathrm{e}^{\mathrm{SPEX}}\right\rangle$ is the average of the excitation energies of each event in the CTMC simulation. Similarly, $\left\langle\Delta \mathrm{e}^{\mathrm{DPEX}}\right\rangle$ is the average of the excitation energies of the two electrons. This table also corrects an error in the previous work regarding the energy loss model for SS and DS that inadvertently omitted the ionization potentials from the expressions in Table A of Ref. [1]. Table A here corrects the formula and subsequent tables correct the calculation of the average energy loss.

We also neglected to emphasize for the reader that for SS and DS, the SDCSs are tabulated in the target frame, the frame in which the resulting sampled electron energy and angle are needed for a transport simulation of the secondary electrons. However, the contribution of the sampled electron energy to the energy loss of the projectile is needed in the projectile frame. Relatively straightforward transformation yields this required energy, as demonstrated in Appendix B for the convenience of the user of the tabulated SDCSs.

To summarize for clarity, a transport simulation may use either the average energy losses for each process, as given in Tables 111 , which implements the energy loss models given in Table $\mathrm{A}$, or

\section{Table A}

Summary of the energy loss models for each reaction channel considered. The models for SI, TI, DI, DCAI, SC, DC, and TEX are the same as in the previous work [1], but for SS and DS are corrected as explained in the text. Energy loss models for SPEX and DPEX are as derived in the present work. For any of the SIM processes (e.g., SI+SPEX), the energy loss is given by the sum of the energy loss for the target and the projectile process. Simulations using the SDCSs can use a distribution of energy losses where the average in this table is replaced by a sampled value from the differential cross section. $\mathrm{IP}_{1,2}(q)$ is the first or second ionization potential of $\mathrm{O}^{q+}$.

\begin{tabular}{ll}
\hline Reaction & Energy loss \\
\hline TEX & $\Delta \mathrm{e}^{\mathrm{TEX}}=7.7 \mathrm{eV}$ \\
SI & $\Delta \mathrm{e}^{\mathrm{SI}}=\mathrm{IP}_{1}\left(\mathrm{H}_{2}\right)+\left\langle\mathrm{E}_{e}^{\mathrm{SI}}(\mathrm{E})\right\rangle$ \\
DI & $\Delta \mathrm{e}^{\mathrm{DI}}=\mathrm{IP}_{1}\left(\mathrm{H}_{2}\right)+\mathrm{IP}_{2}\left(\mathrm{H}_{2}\right)+\left\langle\mathrm{E}_{e}^{\mathrm{DI}}(\mathrm{E})\right\rangle$ \\
SC & $\Delta \mathrm{e}^{\mathrm{SC}}=\left\langle\Delta \mathrm{e}^{\mathrm{SC}}(\mathrm{E})\right\rangle$ \\
DC & $\Delta \mathrm{e}^{\mathrm{DC}}=\left\langle\Delta \mathrm{e}^{\mathrm{DC}}(\mathrm{E})\right\rangle$ \\
TI & $\Delta \mathrm{e}^{\mathrm{TI}}=\mathrm{IP}_{1}\left(\mathrm{H}_{2}\right)+\left\langle\mathrm{E}_{e}^{\mathrm{TI}}(\mathrm{E})\right\rangle+\left\langle\Delta \mathrm{e}^{\mathrm{Tl}}(\mathrm{E})\right\rangle$ \\
DCAI & $\left\langle\Delta \mathrm{e}^{\mathrm{DCAI}}(\mathrm{E})\right\rangle$ \\
SS & $\Delta \mathrm{e}^{\mathrm{SS}}(\mathrm{q})=\left\langle\Delta \mathrm{e}^{\mathrm{SS}}(\mathrm{E})\right\rangle+\mathrm{IP}_{1}(q)$ \\
DS & $\Delta \mathrm{e}^{\mathrm{DS}}=\left\langle\Delta \mathrm{e}^{\mathrm{DS}}(\mathrm{E})\right\rangle+\mathrm{IP}_{1}(q)+\mathrm{IP}_{2}(q)$ \\
SPEX & $\left\langle\Delta \mathrm{e}^{\mathrm{SPEX}}\right\rangle$ \\
DPEX & $\left\langle\Delta \mathrm{e}^{\mathrm{DPEX}}\right\rangle$ \\
\hline
\end{tabular}

could sample the SDCSs as given in Tables 12-41, and implement the energy loss formulas as given in Table A substituting the sampled electron energy for the average electron energy. For the former, all information required is given in Tables 1-11. For the latter, the list below makes explicit the process to follow.

- TEX: The energy loss in the present model is a constant as given in Table $A$.

- SI: Sampling the SDCS as a function of ejected electron energy yields a weighted Monte Carlo instance of the electron energy, which, when added to the first ionization potential of $\mathrm{H}_{2}$ yields the energy loss for that NSIM event (or the SI energy loss component of a SIM event such as SI+SPEX).

- DI: The procedure is as for SI but adding both the first and second ionization potentials to the sampled electron energy.

- SC: The energy loss is modeled by the average energy loss given in Tables 4-11.

- DC: The energy loss is modeled by the average energy loss given in Tables 5-11.

- TI: The energy loss is given by the sampled electron energy from the SDCS, plus the first ionization potential of $\mathrm{H}_{2}$, plus the average energy loss or gain from the charge transfer as given in Table 4 of Ref. [1] for impact energies up to $2 \mathrm{MeV} / \mathrm{u}$ and as extended here in Table B for energies of 5-25 Mev/u.

- DCAI: The energy loss is modeled by sampling the SDCS as a function of ejected electron energy or by using the average energy loss given in Tables 5-11.

- SS: The energy loss is given by the sampled value of the SDCS transformed into the projectile frame as described above and in Appendix B or by use of the average value given in Table 1.

- DS: The same procedure to obtain the energy loss as for SS is used except for the DS SDCS again using the transformation given in Appendix B or by use of the average value given in Table 1.

- SPEX: The energy loss is modeled by the average energy loss given in Table 2.

- DPEX: The energy loss is modeled by the average energy loss given in Table 2.

\subsection{Description of data tables}

Table 1 contains the computed SS and DS integral cross sections along with the associated average energy losses as included in the 
Table B

The energy loss from charge transfer in the TI process for impact energies 5, 10 and $25 \mathrm{MeV} / \mathrm{u}$. This table augments Table 4 of Ref. [1] that gave this quantity for energies below $5 \mathrm{MeV} / \mathrm{u}$.

\begin{tabular}{llll}
\hline Energy $(\mathrm{MeV} / \mathrm{u})$ & $\left\langle\Delta e^{T I}(\mathrm{E})\right\rangle(\mathrm{eV})$ & Energy $(\mathrm{MeV} / \mathrm{u})$ & $\left\langle\Delta e^{T I}(\mathrm{E})\right\rangle(\mathrm{eV})$ \\
\hline $\mathrm{O}^{+}+\mathrm{H}_{2}$ & & $\mathrm{O}^{5+}+\mathrm{H}_{2}$ & \\
\hline 5 & 2715 & 5 & 2642 \\
10 & 5438 & 10 & 5365 \\
25 & 13607 & 25 & 13534 \\
\hline $\mathrm{O}^{2+}+\mathrm{H}_{2}$ & & $\mathrm{O}^{6+}+\mathrm{H}_{2}$ & \\
\hline 5 & 2702 & 5 & 2610 \\
10 & 5425 & 10 & 5333 \\
25 & 13594 & 25 & 13502 \\
\hline $\mathrm{O}^{3+}+\mathrm{H}_{2}$ & & $\mathrm{O}^{7+}+\mathrm{H}_{2}$ & \\
\hline 5 & 2685 & 5 & 2568 \\
10 & 5408 & 10 & 1781 \\
25 & 13577 & 25 & 12950 \\
\hline $\mathrm{O}^{4+}+\mathrm{H}_{2}$ & & $\mathrm{O}^{8+}+\mathrm{H}_{2}$ & \\
\hline 5 & 2665 & 5 & 4591 \\
10 & 5388 & 10 & 12760 \\
\hline 25 & 13557 & 25 & \\
\hline
\end{tabular}

previous work [1]. Similarly, results of the new calculations for SPEX and DPEX are displayed in Table 2 that analogously contains the integral cross sections and average energy losses. The integral cross sections for these processes are not directly required in transport simulations that are based on the SIM processes as they are accounted for within the integral cross sections for the simultaneous target and projectile processes. They are tabulated here for reference or other use, for example, in NSIM transport simulations used to explore the modification of results when the simultaneous processes are taken into account. The tabulated energy losses may be used in either NSIM- or SIM-processes transport simulations. The calculations underlying these cross sections and average energy losses also yielded the $b_{\max }$ for each process and impact energy required to subsequently implement the present model yielding the SIM-processes integral cross sections.

In these and subsequent tables we indicate by a footnote a small number of corrections to the previously published integral cross sections or average energy losses. For example, in compiling the present work we have noted a few typographical errors in the previous tables and also have made additional calculations where the Monte Carlo uncertainty was high or a cross section or energy loss was statistically uncertain enough not to be included. In addition, in order to have no "zeroes" in the data set (caused by the inability to compute the cross section with sufficient statistical uncertainty in a reasonable computational time) to ease the data handling and decision making in implementing transport simulations, we have extrapolated the cross sections or average energy losses, consistent with known behaviors, to provide a non-zero entry in the table. Such cases are also indicated via a footnote. Here for all reaction channels, we have extended the range of impact energies considered up to $25000 \mathrm{keV} / \mathrm{u}$ (adding data for 5000 , 10000 , and $25000 \mathrm{keV} / \mathrm{u}$ ) owing to the fact that measurements of the stopping power, against which to test the data set, exist at energies much greater than $2000 \mathrm{keV} / \mathrm{u}$.

Tables 3-11 display the results of the present model for the SIM processes for each ionization stage of oxygen, respectively. Shown are the integral cross sections for each process for each impact energy along with the associated average energy loss. In the previous work [1] we instead tabulated "components" of the energy loss, for example, the average ejected electron energy, from which the user of the data could assemble the corresponding average energy loss from the model given in Table A. Here, particularly owing to the fact that the average energy loss for the SIM processes is composed of both target and projectile-process contributions, we have found that it is more convenient to simply compute the complete average energy loss for each process by assembling all of the components rather than requiring the user to bring together information from multiple tables along with other auxiliary data (i.e., ionization potentials).

If the average energy loss for the individual, NSIM processes is of interest directly, it can be obtained from the tables as well. In particular, for the projectile processes it is given in Tables 1 and 2. For the NSIM target processes, it is simply the average energy loss given for the target-only process within the list of SIM processes (e.g., SI from within the SIM-processes group SI, SI+SS, SI+DIS, SI+SPEX, and SI+DPEX). In addition, we note that the NSIM integral cross sections are also given in the table; they are denoted, for example, "SI sum", meaning the sum of cross sections for the SIM processes SI, SI+SS, SI+DS, SI+SPEX, and SI+DPEX, reflecting the fact that the SIM-process cross sections are simply the partitioned components of the calculated NSIM cross section.

The SDCSs as a function of ejected electron energy and angle for 1-2000 keV/u tabulated in Ref. [1] for the NSIM processes should still be used, as described here, for the SIM processes through addition. Here, Tables 12 through 41 display the newly computed SDCSs for the ejected-electron producing NSIM processes (i.e., for those processes leading to one or two ejected electrons) for 5000 , 10000 , and $25000 \mathrm{keV} / \mathrm{u}$. For these higher energies, the ionization processes involving electron capture, TI and DCAI, are increasingly less probable, and so SDCSs are therefore increasingly difficult to calculate in a reasonable time with the CTMC method. Therefore, for $\mathrm{TI}$, we have greatly reduced the number of ejected electron energy and angle bins to achieve acceptable statistics in this Monte Carlo approach for the very small cross sections, as seen in the corresponding tables. For DCAI, the cross sections are too small to compute within reasonable computational times and, as reflected in the integral cross section tables where all DCAI cross sections are obtained by extrapolation from lower energies, no sufficient number of Monte Carlo events were produced to allow tabulation of SDCSs. We also note for clarity that the SS and DS SDCSs are tabulated in the laboratory frame, as are the target ionization cross sections, since ion-transport simulations will most likely account for and propagate all ejected electrons in that frame.

\section{Simulation results: Stopping power, charge state fractions, and equilibrium charge state}

\subsection{Stopping power}

As we did in the previous work [1] employing just the NSIM processes, we have used a Monte Carlo ion-trajectory simulation to determine the stopping power as a function of impact energy using the new, full set of SIM processes. The result is shown in Fig. 1 in comparison with the previous NSIM-processes result. Also for comparison, the existing measurements of the stopping power, clustered around 3-10 $\mathrm{keV} / \mathrm{u}$ and around $25-90 \mathrm{MeV} / \mathrm{u}$, are displayed as is the likely most reliable recommended value of the stopping power, Stopping and Range of Ions in Matter (SRIM) 2013 [29]. As is apparent from the figure, the SIM processes yield a stopping power curve much more in agreement with the SRIM recommendation than did the previous NSIM processes alone. Furthermore, inclusion of the simultaneous processes does not change the previous good agreement at low $(\sim 10-100 \mathrm{keV} / \mathrm{u})$ or high ( $\geq 2000 \mathrm{keV} / \mathrm{u})$ impact energy.

Examining the integral cross sections and average energy losses shown in the tables, we can understand why this agreement at low and high energies is maintained. At low collision energy, stripping is relatively unlikely and, since the charge state is low, projectile excitation occurs with only very small energy loss, so 


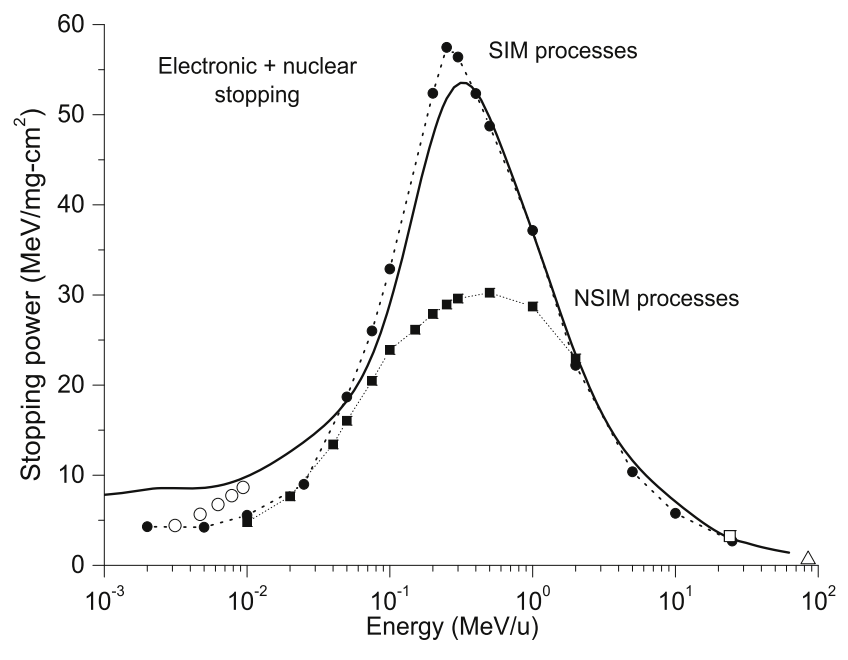

Fig. 1. Comparison of the present calculation (simultaneous electronic transitions on target and projectile - SIM processes) of the electronic plus nuclear stopping power for oxygen ions passing through $\mathrm{H}_{2}$ gas (circles) with the previous calculation (non-simultaneous electronic transitions on the target and projectile - NSIM processes) [1] (squares), accepted values from SRIM 2013 (Stopping and Range of Ions in Matter, version 2013) [29] (solid line), and the measurements by Hvelplund [36] (open squares), Herault et al. [37] (open circles), and Bimbot et al. [38] (open triangle).

the simultaneous projectile energy loss processes do not add much to the total energy loss due to target processes. This accounts for the very small difference in this regime between simulations using the NSIM or SIM processes. At high collision energy, the projectile is nearly or fully stripped and so projectile excitation or stripping is very rare (low cross section) even though the energy loss on those events would be high. Consequently the NSIM processes already represent well enough the additional energy loss from SS treated independently, accounting in this regime for the negligible difference between the simulations using the NSIM and SIM processes. In contrast, at intermediate collision energies, the greatest probability occurs for projectile processes and they contribute approximately half the combined target and projectile energy loss, explaining the improved agreement with the recommended stopping power from the SIM-processes simulation compared to the NSIM-processes simulation.

In separate, as of yet unpublished work using the present data in modeling ion precipitation into Jupiter's upper atmosphere, we have also computed the stopping power utilizing sampling of the SDCSs to obtain electron ejection energies and angles for events within the simulation. We find very good agreement of the stopping power with the present ion-transport simulation that uses instead the tabulated average energy losses, indicating consistency of the two methods.

\subsection{Equilibrium charge state}

An additional point of comparison with recommended data can be made, namely comparing the simulated equilibrium charge state as a function of collision energy with semi-empirical models of this quantity developed by Schiwietz and Grande [30] or Dmitriev and Nikoleav [31]. The equilibrium charge state reflects the balance of charge states that develops in sufficiently thick (or dense) targets at a given impact energy, resulting from the equilibration of charge changing collisions (i.e., stripping and charge transfer). At high energy, stripping dominates and therefore the equilibrium charge state is high, whereas at low energy, charge transfer dominates and the charge state is low.

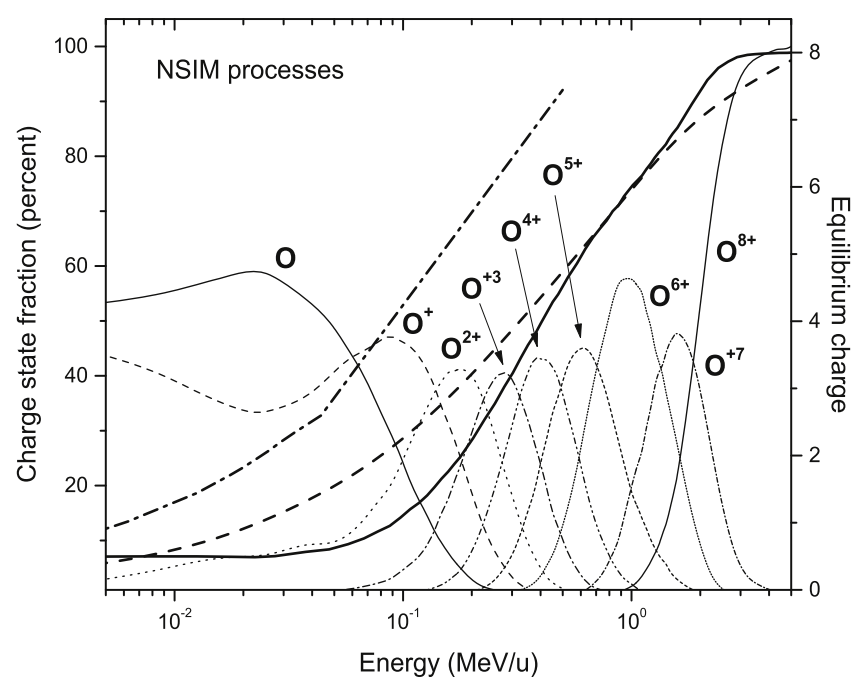

Fig. 2. The fractional population of each ionization stage (left vertical axis) as a function of impact energy for the NSIM-processes (non-simultaneous electronic transitions on the target and projectile) model along with the equilibrium charge state (heavy solid line) as a function of the impact energy (right vertical axis) compared with the empirical equilibrium charge state models of Schiwietz and Grande [30] (heavy dashed line) and Dmitriev and Nikoleav [31] (heavy dasheddotted line).

Figs. 2 and 3 (right hand axis) show the results from our NSIMand SIM-processes simulations, respectively, for the equilibrium charge state as a function of impact energy compared with that from the Schiwietz and Grande (SG) and the Dmitriev and Nikoleav (DN) semi-empirical models. Unlike the SRIM recommended stopping power displayed here, which is specifically for oxygen transport through molecular hydrogen, the semi-empirical model of SG is a fit to more than 500 data points tabulated for a variety of projectile species and target gases. In fact, this semi-empirical fit is dependent on the nuclear charge of the gas target (ranging from $\left.Z_{t}=1-54\right)$, not distinguishing atomic and molecular species, so here we have adopted a value of $Z_{t}=1$. The SG model has the advantage that it is based on a very large number of experimental data, but it is not made specifically for oxygen passing through $\mathrm{H}_{2}$. In contrast, the DN model was created when a much smaller amount of data was available, but it is based on incident ions with nuclear charge less than 10 and has parameters adapted for $\mathrm{H}_{2}$ targets, thus being very relevant for the present collision system.

The SG model fits the NSIM results well for high energies, but overestimates it for lower energies, while the DN model is significantly higher than the NSIM results throughout the whole range of energies considered. In contrast, the SG model significantly underestimates the equilibrium charge state predicted by the SIMprocesses results for intermediate to high energies, but agrees well with it for low energies, while the DN model is relatively closer to the SIM results and the NSIM prediction throughout the energy range considered. Therefore, it is difficult to draw a firm conclusion that the semi-empirical formulas support either the NSIM- or SIMprocesses model, but rather simply generally agree with them both, differing as noted above.

\subsection{Charge state fractions}

Also shown in these figures (left hand axis) is the distribution of charge states that exist as a function of impact energy. That is, at a given energy, though an equilibrium charge is established in sufficiently thick (or dense) targets, there exists a distribution of charge states averaging to this value. Comparing the results of the NSIM- and SIM-processes simulations, two significant differences 




Fig. 3. The fractional population of each ionization stage (left vertical axis) as a function of impact energy for the SIM-processes (simultaneous electronic transitions on the target and projectile) model along with the equilibrium charge state (heavy solid line) as a function of the impact energy (right vertical axis) compared with the empirical equilibrium charge state models of Schiwietz and Grande [30] (heavy dashed line) and Dmitriev and Nikoleav [31] (heavy dashed-dotted line).

stand out. First, the peaks of the ion fraction curves are shifted to lower energies for the SIM-processes result in comparison with those for the NSIM-processes simulation. For example, the peak of the distribution for $\mathrm{O}^{6+}$ shifts from about $1 \mathrm{MeV} / \mathrm{u}$ for the NSIM processes to about $400 \mathrm{keV} / \mathrm{u}$ for the SIM-processes simulation results. Second, while in both cases the $\mathrm{O}^{6+}$ fraction attains the largest value of any of the charge states other than $\mathrm{O}^{8+}$ at the high impact energies, it is relatively larger compared to the other nearby charge states in the SIM-processes simulation (though not much changed in peak magnitude - about 55\% for the NSIM processes and about $62.5 \%$ for the SIM processes).

For oxygen passing through $\mathrm{H}_{2}$, there exist measurements by Østgaard Olsen and Hvelplund [32] and Jorgensen et al. [33] of the charge state fractions, but only at very low energies (i.e., below $31.25 \mathrm{keV} / \mathrm{u}$ ). These data are compared with the results of the NSIM- and SIM-processes simulations in Figs. 4 and 5, respectively. Of significant note, the measurements indicate that at the lower energies considered, production of $\mathrm{O}^{-}$becomes appreciable, a channel not represented in present calculations and data set. Careful examination of these figures shows that, particularly for $\mathrm{O}$ and $\mathrm{O}^{+}$, the data agree better with the SIM-processes results, though good quantitative agreement is not present in either case.

Consider, for example, how the data for $\mathrm{O}$ and $\mathrm{O}^{+}$compare with the NSIM results. At about $30 \mathrm{keV} / \mathrm{u}$, the measured fraction for $\mathrm{O}$ is about a factor of three below the simulation results and at about $3 \mathrm{keV} / \mathrm{u}$ they are larger by almost a factor of two. For the $\mathrm{O}^{+}$fraction, the measurements are larger than the simulation results at $30 \mathrm{keV} / \mathrm{u}$ and almost a factor of five below them at 3 $\mathrm{keV} / \mathrm{u}$. In contrast, the measured $\mathrm{O}$ fraction is in good agreement with the SIM-processes simulation for 30 to almost $10 \mathrm{keV} / \mathrm{u}$ but grow significantly larger down to $3 \mathrm{keV} / \mathrm{u}$. For the $\mathrm{O}^{+}$fraction, while they do not agree quantitatively with the simulation results, they follow much more the trend of the SIM-model results than for the NSIM results. Uncertainties in the CTMC results for energies below $10 \mathrm{keV} / \mathrm{u}$ that certainly grow and the lack of inclusion of the negative ion formation channel in this very low energy regime likely contribute to the deviations of the present results from the measurements.



Fig. 4. The fractional population for ionization stages $-1,0,1$, and 2 as a function of impact energy for the NSIM-processes (non-simultaneous electronic transitions on the target and projectile) model along with the measurements of Østgaard Olsen and Hvelplund [32] (filled symbols) and Jorgensen et al. [33] (open symbols).

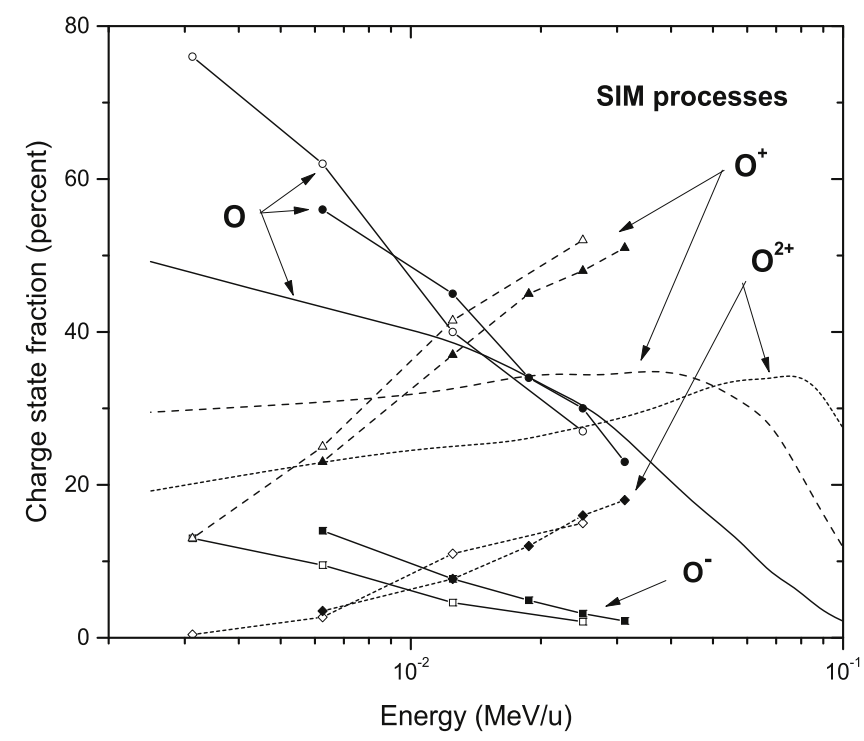

Fig. 5. The fractional population for ionization stages $-1,0,1$, and 2 as a function of impact energy for the SIM-processes (simultaneous electronic transitions on the target and projectile) model along with the measurements of Østgaard Olsen and Hvelplund [32] (filled symbols) and Jorgensen et al. [33] (open symbols).

\subsection{Auxiliary data model}

To determine if this comparison indicates some significant inaccuracy of the present data set or its inadequacy by neglecting the production and destruction of $\mathrm{O}^{-}$, we have created an auxiliary data model for the integral cross sections needed to describe the negative ion channel in our simulation. Calculation of these cross sections presents an especially difficult challenge to the CTMC approach given the very delicate binding of the outmost electron in $\mathrm{O}^{-}$, but, fortunately, certain key data for the relevant channels exist to help guide assembly of the required auxiliary data.

Key among the existing data is the negative ion formation cross section measured by Lindsay et al. [34] for $\mathrm{O}+\mathrm{H}_{2}$ for energies between 2 and $5 \mathrm{keV}$, by Fogel et al. [35] (2.5-6 keV), by Østgaard 
Table C

Auxiliary data model for negative ion formation in $\mathrm{O}+\mathrm{H}_{2}$ collisions for energies between 1 and $25000 \mathrm{keV} / \mathrm{u}$ interpolated or extrapolated from the measurements of the integral cross section from Refs. [32-35].

\begin{tabular}{lll}
\hline Energy $(\mathrm{keV} / \mathrm{u})$ & Cross section $\left(\mathrm{cm}^{2}\right)$ & Energy loss $(\mathrm{eV})$ \\
\hline 1 & $5.0 \mathrm{E}-17$ & 14.5 \\
10 & $1.9 \mathrm{E}-16$ & 19.4 \\
50 & $4.5 \mathrm{E}-17$ & 41.2 \\
75 & $3.0 \mathrm{E}-17$ & 54.8 \\
100 & $1.1 \mathrm{E}-17$ & 68.4 \\
200 & $1.0 \mathrm{E}-17$ & 123. \\
500 & $3.5 \mathrm{E}-18$ & 286. \\
1000 & $1.6 \mathrm{E}-18$ & 559. \\
2000 & $7.0 \mathrm{E}-19$ & 1103. \\
5000 & $2.0 \mathrm{E}-19$ & 2737. \\
10000 & $8.0 \mathrm{E}-20$ & 5460. \\
25000 & $2.0 \mathrm{E}-20$ & 13629. \\
\hline
\end{tabular}

Table D

Auxiliary data model for SIM processes in the negative ion channel as described in the text.

\begin{tabular}{|c|c|c|c|}
\hline Channel & $\begin{array}{l}\text { Cross section }\left(\mathrm{cm}^{2}\right) \text {, } \\
\text { energy loss }(\mathrm{eV})\end{array}$ & & \\
\hline Energy & 1 & 10 & 50 \\
\hline SI & $1.07 \mathrm{E}-16,30.4$ & $9.94 \mathrm{E}-18,36.0$ & $1.14 \mathrm{E}-16,60.3$ \\
\hline $\mathrm{SI}+\mathrm{SS}$ & $1.66 \mathrm{E}-16,51.4$ & $1.07 \mathrm{E}-15,60.0$ & $2.44 \mathrm{E}-15,105$. \\
\hline SI Sum & $2.73 \mathrm{E}-16$ & $1.08 \mathrm{E}-15$ & $2.55 \mathrm{E}-15$ \\
\hline DI & $1.00 \mathrm{E}-20,154$ & $7.14 \mathrm{E}-20,96.5$ & $8.56 \mathrm{E}-19,151$. \\
\hline $\mathrm{DI}+\mathrm{SS}$ & $1.19 \mathrm{E}-17,175$. & $1.90 \mathrm{E}-16,121$ & $8.98 \mathrm{E}-16,196$. \\
\hline DI Sum & $1.19 \mathrm{E}-17$ & $1.90 \mathrm{E}-16$ & $8.99 \mathrm{E}-16$ \\
\hline TEX & $2.41 \mathrm{E}-19,7.7$ & $5.20 \mathrm{E}-18,7.7$ & $7.01 \mathrm{E}-17,7.7$ \\
\hline $\mathrm{TEX}+\mathrm{SS}$ & $3.18 \mathrm{E}-18,34.7$ & $1.23 \mathrm{E}-15,31.7$ & $1.12 \mathrm{E}-15,52.6$ \\
\hline \multirow[t]{2}{*}{ TEX Sum } & $3.19 \mathrm{E}-16$ & $1.24 \mathrm{E}-15$ & $1.19 \mathrm{E}-15$ \\
\hline & 75 & 100 & 200 \\
\hline SI & $9.67 \mathrm{E}-17,80.0$ & $7.54 \mathrm{E}-17,97,8$ & $6.09 \mathrm{E}-17,145$. \\
\hline $\mathrm{SI}+\mathrm{SS}$ & $2.47 \mathrm{E}-15,131$ & $2.43 \mathrm{E}-15,152$ & $2.11 \mathrm{E}-15,204$. \\
\hline SI Sum & $2.57 \mathrm{E}-15$ & $2.50 \mathrm{E}-15$ & $2.17 \mathrm{E}-15$ \\
\hline DI & $4.61 \mathrm{E}-19,201$ & $1.64 \mathrm{E}-19,246$ & $1.53 \mathrm{E}-19,362$. \\
\hline $\mathrm{DI}+\mathrm{SS}$ & $9.72 \mathrm{E}-16,252$. & $9.39 \mathrm{E}-16,300$ & $7.03 \mathrm{E}-16,303$. \\
\hline DI Sum & $9.72 \mathrm{E}-16$ & $9.39 \mathrm{E}-16$ & $7.03 \mathrm{E}-16$ \\
\hline TEX & $6.88 \mathrm{E}-17,7.7$ & $6.03 \mathrm{E}-17,7.7$ & $6.11 \mathrm{E}-17,7.7$ \\
\hline TEX+SS & $1.05 \mathrm{E}-15,58.2$ & $1.02 \mathrm{E}-15,61.5$ & $9.39 \mathrm{E}-16,66.5$ \\
\hline \multirow[t]{2}{*}{ TEX Sum } & $1.12 \mathrm{E}-15$ & $1.08 \mathrm{E}-15$ & $1.00 \mathrm{E}-15$ \\
\hline & 500 & 1000 & 2000 \\
\hline SI & $2.93 \mathrm{E}-17,189$ & $1.64 \mathrm{E}-17,203$. & $5.32 \mathrm{E}-18,205$. \\
\hline $\mathrm{SI}+\mathrm{SS}$ & $1.64 \mathrm{E}-15,250$ & $1.28 \mathrm{E}-15,264$ & $9.70 \mathrm{E}-16,264$. \\
\hline SI Sum & $1.67 \mathrm{E}-15$ & $1.30 \mathrm{E}-15$ & $9.75 \mathrm{E}-16$ \\
\hline DI & $1.41 \mathrm{E}-20,466$ & $7.02 \mathrm{E}-21,485$ & $3.00 \mathrm{E}-21,467$. \\
\hline $\mathrm{DI}+\mathrm{SS}$ & $3.83 \mathrm{E}-16,527$ & $2.23 \mathrm{E}-16,546$ & $1.22 \mathrm{E}-16,526$ \\
\hline DI Sum & $3.83 \mathrm{E}-16$ & $2.23 \mathrm{E}-16$ & $1.22 \mathrm{E}-16$ \\
\hline TEX & $2.54 \mathrm{E}-17,7.7$ & $9.90 \mathrm{E}-18,7.7$ & $1.22 \mathrm{E}-18,7.7$ \\
\hline TEX+SS & $7.88 \mathrm{E}-16,68.4$ & $6.58 \mathrm{E}-16,68.2$ & $5.31 \mathrm{E}-16,66.7$ \\
\hline \multirow[t]{2}{*}{ TEX Sum } & $8.13 E-16$ & $6.68 \mathrm{E}-16$ & $5.32 \mathrm{E}-16$ \\
\hline & 5000 & 10000 & 25000 \\
\hline SI & $3.27 \mathrm{E}-18,192$ & $2.02 \mathrm{E}-18,182$ & $6.55 \mathrm{E}-19,168$. \\
\hline $\mathrm{SI}+\mathrm{SS}$ & $6.28 \mathrm{E}-16,250$ & $4.35 \mathrm{E}-16,241$ & $2.51 \mathrm{E}-16,226$. \\
\hline SI Sum & $6.31 \mathrm{E}-16$ & $4.37 \mathrm{E}-16$ & $2.52 \mathrm{E}-16$ \\
\hline DI & $1.05 \mathrm{E}-21,420$ & $4.70 \mathrm{E}-22,397$. & $1.70 \mathrm{E}-22,336$. \\
\hline $\mathrm{DI}+\mathrm{SS}$ & $4.98 \mathrm{E}-19,478$ & $2.36 \mathrm{E}-19,456$ & $8.00 \mathrm{E}-18,394$. \\
\hline DI Sum & $4.98 \mathrm{E}-17$ & $2.36 \mathrm{E}-17$ & $8.00 \mathrm{E}-18$ \\
\hline TEX & $2.34 \mathrm{E}-19,7.7$ & $9.66 \mathrm{E}-20,7.7$ & $9.00 \mathrm{E}-21,7.7$ \\
\hline TEX+SS & $3.86 \mathrm{E}-18,65.5$ & $2.87 \mathrm{E}-18,66.3$ & $1.84 \mathrm{E}-18,65.7$ \\
\hline TEX Sum & $3.86 \mathrm{E}-18$ & $2.87 \mathrm{E}-18$ & $1.84 \mathrm{E}-18$ \\
\hline
\end{tabular}

Olsen and Hvelplund [32] (100-500 keV), and Jorgensen et al. [33] (50-400 keV). Interpolating and extrapolating these data we have estimated the integral cross section for the present set of energies $(1-25000 \mathrm{keV} / \mathrm{u})$ as displayed in Table C. We have also estimated the energy loss using the model we have employed for SC for the oxygen ions (here, single capture of an electron from $\mathrm{H}_{2}$ by neutral oxygen). This process augments those possible for $\mathrm{O}+\mathrm{H}_{2}$ (Table 3) and we make the reasonable assumption that no simultaneous projectile process (SS, DS, SPEX, or DPEX) occur with significant probability. That is, since the negative ion cannot exist with a core excited state, neither simultaneous SPEX or DPEX is possible and since the outer electron is so weakly bound, it is not likely that during SS or DS an electron would be captured to this weakly bound state.

Also needed is a data model for the processes involving $\mathrm{O}^{-}+\mathrm{H}_{2}$. Among the projectile processes in this case, $\mathrm{SS}$ will be the dominant one owing to the very small binding energy of the negative ion, and we assume this dominance is great enough to neglect DS. As mentioned above, SPEX and DPEX are not possible while maintaining the binding of the outer electron. Therefore the possible SIM processes are SI, SI+SS, DI, DI+SS, TEX, and TEX+SS. We approximate these by adopting the present $\mathrm{O}+\mathrm{H}_{2}$ results (Table 3) multiplied by a factor of ten. This factor is determined by comparing the SS of $\mathrm{O}$ and $\mathrm{O}^{-}$by $\mathrm{H}_{2}$ as measured by Lindsay et al. [34]. It also assumes that processes involving capture (i.e., $\mathrm{SC}, \mathrm{TI}$ ) are suppressed for $\mathrm{O}^{-}$ impact as they are in the case of $\mathrm{O}$ impact compared to $\mathrm{O}^{+}$impact that otherwise might be used as the baseline for the model since $\mathrm{O}^{-}$and $\mathrm{O}^{+}$are both singly charged. The resulting model data set is displayed in Table $\mathrm{D}$.

Augmenting the present data set (Tables 1-11) with this auxiliary data (Tables $C$ and D) and repeating our ion-transport simulation we find significantly improved agreement with the measured ion-fraction distributions, as illustrated in Fig. 6. In particular, the ion fraction for $\mathrm{O}^{-}$that results agrees very well with both of the measurements from the lowest energy considered up to about 50 $\mathrm{keV} / \mathrm{u}$ where this channel becomes negligible. The agreement of the results also improve below about $12 \mathrm{keV} / \mathrm{u}$ for the fractional population of $O$ produced in the simulation compared with the measurements. For $\mathrm{O}^{+}$and $\mathrm{O}^{2+}$, while the simulation including the auxiliary data does not achieve as close a quantitative agreement with the measurements, it significantly improves the trend of results. That is, comparing Figs. 5 and 6, one can readily see that the augmented simulation results reflect the shape of the $\mathrm{O}^{+}$and $\mathrm{O}^{2+}$ measurements much better from about $30 \mathrm{keV} / \mathrm{u}$ and below. The remaining differences between the simulation results and the measurements therefore likely represent the limit of accuracy of the present data in the low energy regime. Without a comprehensive set of benchmark data for the ionization, capture, and stripping cross sections and energy losses for the charge states $q=-1,0,1,2$, and 3 in this regime, an ab initio adjustment of the data to yield potentially better agreement with the measurements is not possible.

To show that the augmented data set not only improves the simulated ion fractions at low energy but also does not adversely affect the existing reasonable agreement with the recommended stopping power, in Fig. 7 we compare the results of the simulation using both the SIM-processes data set and including the auxiliary data with the SRIM recommended stopping power and the available measurements in that energy regime. As the figure shows, results using the augmented data set agree somewhat more closely with the recommended curve, but agree on average about as well as before with the measurements. With this check on the energy loss predicted by the simulation using the augmented data set, it is clear that inclusion of the negative ion channel is important and that the auxiliary data model adopted here is reasonable.

\section{Summary and conclusions}

We have presented here a new data set assembled by considering both non-simultaneous and simultaneous projectile and target processes for $1-25000 \mathrm{keV} / \mathrm{u} \mathrm{O}^{q+}, q=0-8$ colliding with $\mathrm{H}_{2}$. This has been accomplished by creating a new model of the simultaneous processes based on partitioning the non-simultaneous cross sections using probability as a function of impact parameter 


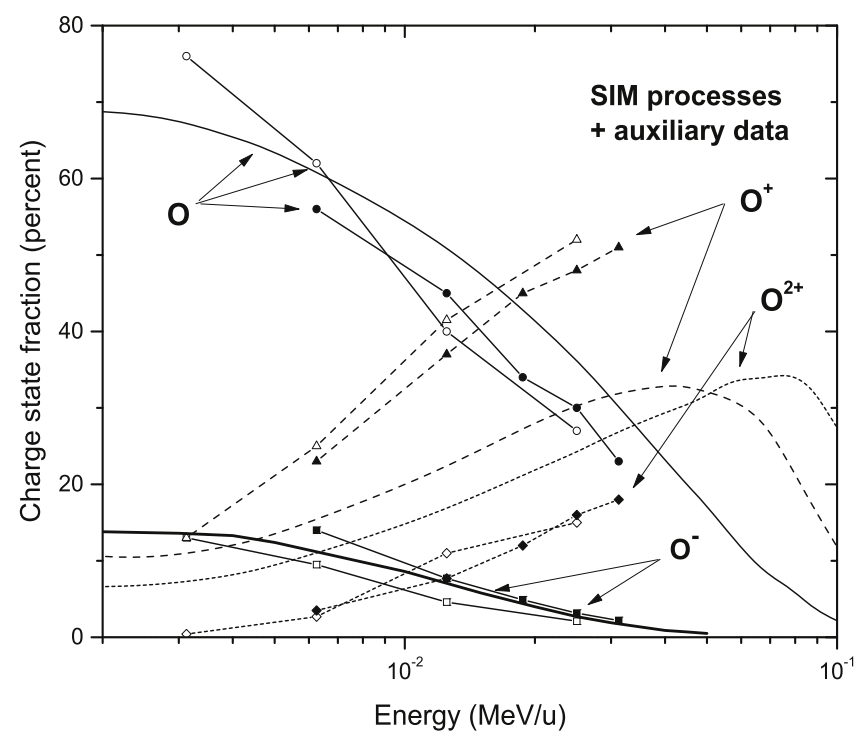

Fig. 6. The fractional population for ionization stages $-1,0,1$, and 2 as a function of impact energy for the SIM-processes (simultaneous electronic transitions on the target and projectile) model, augmented with the auxiliary data for the negative ion channel described in the text, along with the measurements of Østgaard Olsen and Hvelplund [32] (filled symbols) and Jorgensen et al. [33] (open symbols).

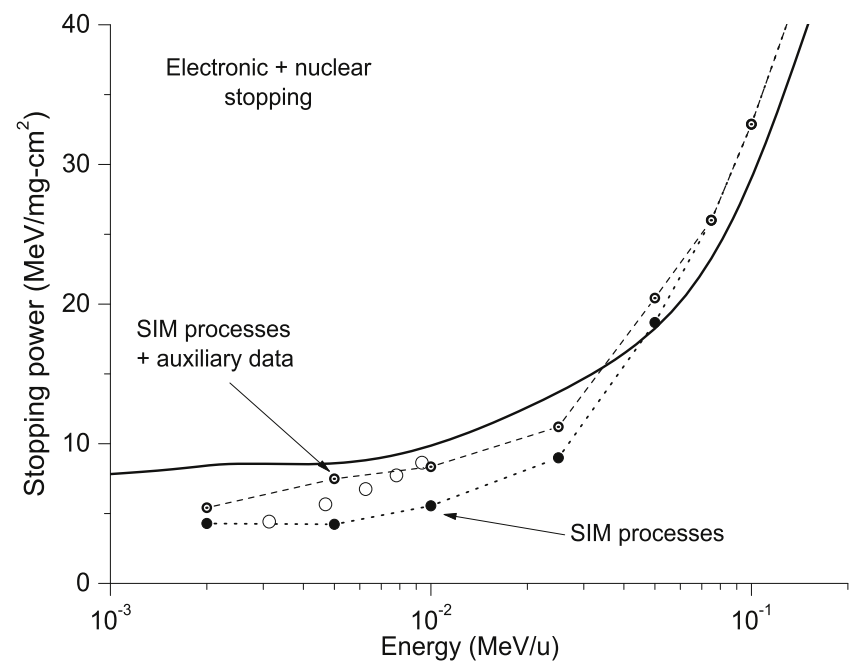

Fig. 7. Comparison of the present calculation of the electronic plus nuclear stopping power for the SIM-processes (simultaneous electronic transitions on the target and projectile) model (circles) and the SIM-processes model augmented with the auxiliary data for the negative ion channel (bulleted open circles) with accepted values from SRIM 2013 (Stopping and Range of Ions in Matter, version 2013) [29] (solid line) and the measurements by Herault et al. [37] (open circles).

distributions from independent active-target-electron and activeprojectile-electron calculations. Validity of the data set has been demonstrated by its use in a Monte Carlo transport simulation that yields improved agreement with the recommended stopping power compared to the previous [1] data set that considered only non-simultaneous target and projectile processes.

From this improved agreement with the recommended stopping power at intermediate collision energy, and the maintenance of the good agreement at both low and high energies, we conclude that a better representation of the energy deposition by oxygen ions precipitating into the upper atmosphere of Jupiter can be obtained with this expanded, SIM-processes set of reactions. Use of this revised data set should also provide a more physically realistic secondary-electron distribution, particularly for intermediate energies, and therefore an improved atmospheric reaction network, improved description of ion contribution to atmospheric currents, and consequently an improved understanding of the Jovian ionosphere-atmosphere coupling.

\section{Acknowledgments}

DRS and TEC gratefully acknowledge support from the NASA Planetary Atmospheres program through grant NNX14AG79G.

\section{Appendix A. Survey of the doubly differential cross section}

As noted in the text, a more physically completed description of the spectrum of secondary electrons is provided by the DDCS compared to that provided by the SDCSs. However, the computational effort to produce the DDCS via the CTMC method is substantially greater. This occurs because computation of the DDCS in the CTMC method requires binning the secondary-electronproducing events into a two-dimensional array as opposed to two, one-dimensional arrays (electron energy and angle separately), requiring a much larger number of events to achieve statistically significant results. Implementation of sampling of the DDCS would also introduce some level of greater complexity in ion-/electrontransport simulations, particularly if they had to deal with statistically noiser DDCSs. Therefore, a trade off in the level of physical completeness of the model of secondary-electron production with computational effort and complexity in CTMC calculations and ion/electron-transport simulation is required.

The more complete physical description inherent in the DDCS is the correlation between ejected-electron energy and angle. For example, in some channels, fast electrons tend to be emitted dominantly in the forward direction, whereas slow electrons tend to be emitted largely isotropically. To illustrate the degree to which the DDCS reflects this energy-angle correlation, and thereby the degree to which the SDCSs do not reflect it, we present here a survey of the DDCS for the secondary-electron-producing channels. Some bias in this survey exists owing to the fact that a sufficient number of events had to exist in our CTMC calculations to enable binning the DDCS with statistics to produce smooth enough curves for clarity of display.

\section{A.1. Single ionization}

Beginning with SI, Figs. A.1 and A.2 show examples of the DDCS for $50 \mathrm{keV} / \mathrm{u} \mathrm{O}^{2+}+\mathrm{H}_{2}$ and $1 \mathrm{MeV} / \mathrm{u} \mathrm{O}^{5+}+\mathrm{H}_{2}$. The Born corrections have been added as applied to the SDCSs and the SDCS as a function of ejected-electron energy (i.e., the DDCS integrated over ejected-electron angle) has been included in the figures for comparison. For $50 \mathrm{keV} / \mathrm{u} \mathrm{O}^{2+}$ impact, there is a strong variation of the DDCS as electron-emission angle changes, and we note the existence of the well-known electron-capture-to-the-continuum cusp for small angles, where the ejected-electron velocity matches the projectile velocity. Overall, the shape of the DDCS curves is not dramatically varying with ejection angle, but increasing in magnitude as angle increases up to intermediate angles and then decreasing. For $1 \mathrm{MeV} / \mathrm{u} \mathrm{O}^{5+}$ impact, the DDCS curves are quite similar in shape and magnitude up to about an ejected-electron energy of $100 \mathrm{eV}$. For higher ejection energies, their behavior diverges and we note the shifting of the well-known binary peak in the spectrum of ejected electrons that shifts in position with ejection angle (i.e., the position of the binary peak for a given projectile velocity, ejection angle, and target ionization potential 


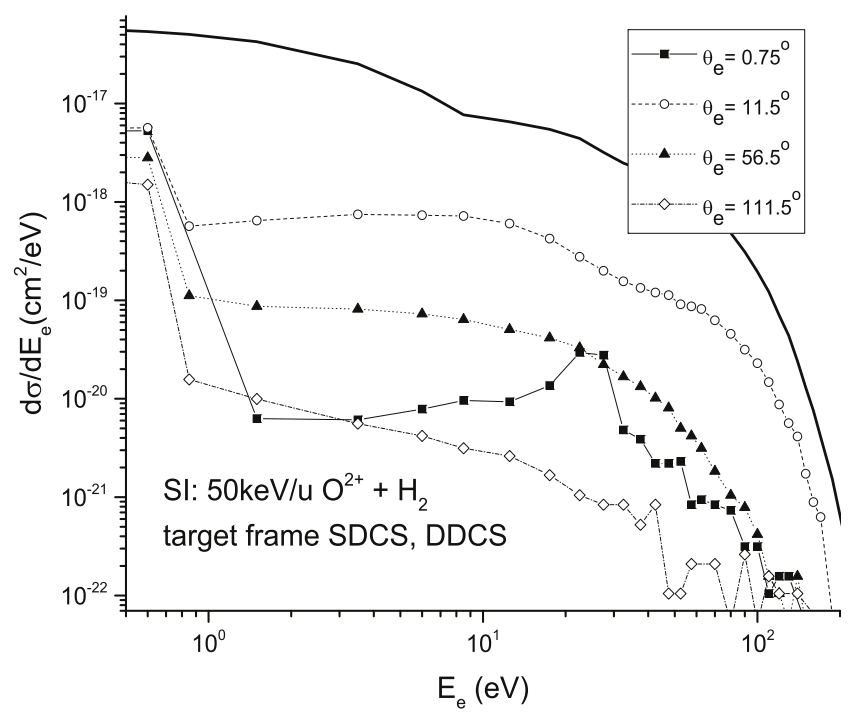

Fig. A.1. The doubly differential cross section for single ionization (SI) in $50 \mathrm{keV} / \mathrm{u}$ $\mathrm{O}^{2+}+\mathrm{H}_{2}$ (curves with symbols for four electron ejection angles as indicated, as a function of ejected electron energy). The solid curve shows the singly differential cross section as a function of electron ejection angle, that is, the doubly differential cross section summed over electron ejection angles, for comparison.

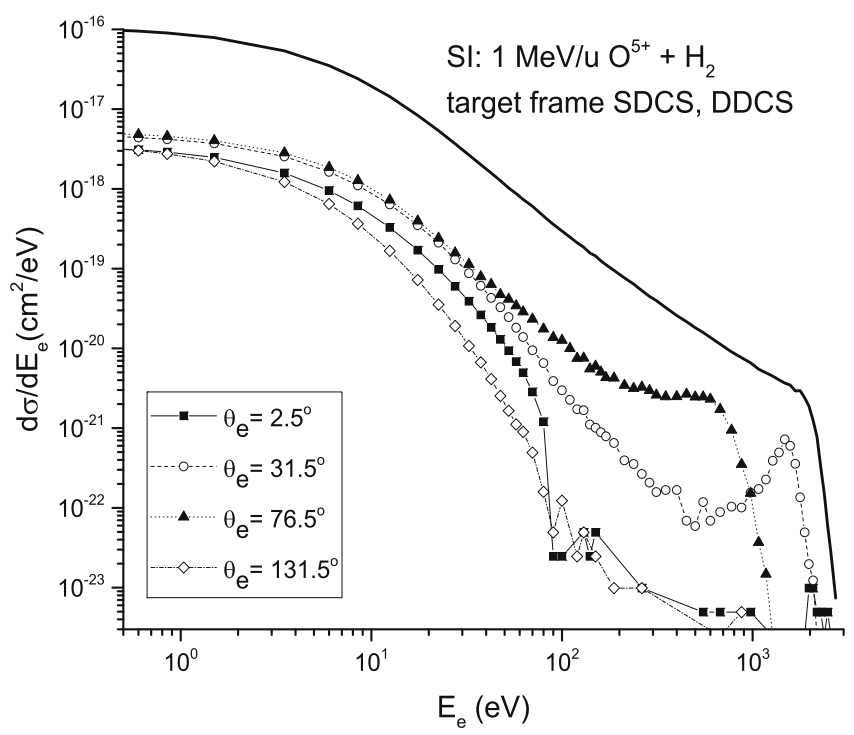

Fig. A.2. The doubly differential cross section for single ionization (SI) in $1 \mathrm{MeV} / \mathrm{u}$ $\mathrm{O}^{5+}+\mathrm{H}_{2}$ (curves with symbols for four electron ejection angles as indicated, as a function of ejected electron energy). The solid curve shows the singly differential cross section as a function of electron ejection angle, that is, the doubly differential cross section summed over electron ejection angles, for comparison.

is given approximately by an electron energy of $2 v_{p r o j}^{2} \cos ^{2}(\theta)$ $-\mathrm{IP})$.

\section{A.2. Transfer ionization}

Fig. A.3, illustrates the behavior of the DDCS for TI in $100 \mathrm{keV} / \mathrm{u}$ $\mathrm{O}^{3+}+\mathrm{H}_{2}$. One sees generally the same variation with ejection angle as for SI, namely, (i) similar shapes to the curves for small electron energies, with increasing magnitude up to intermediate electron angles and then a decrease, and (ii) the presence of a cusp at small angles and significant variation at large electron energies resulting from the behavior of the binary peak.

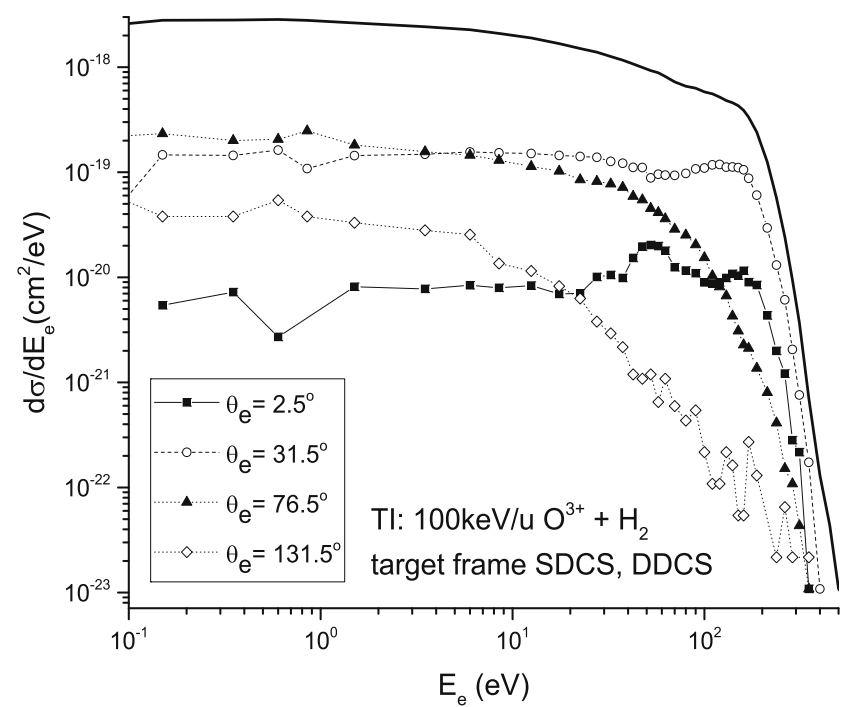

Fig. A.3. The doubly differential cross section for transfer ionization (TI) in 100 $\mathrm{keV} / \mathrm{u} \mathrm{O}^{3+}+\mathrm{H}_{2}$ (curves with symbols for four electron ejection angles as indicated, as a function of ejected electron energy). The solid curve shows the singly differential cross section as a function of electron ejection angle, that is, the doubly differential cross section summed over electron ejection angles, for comparison.

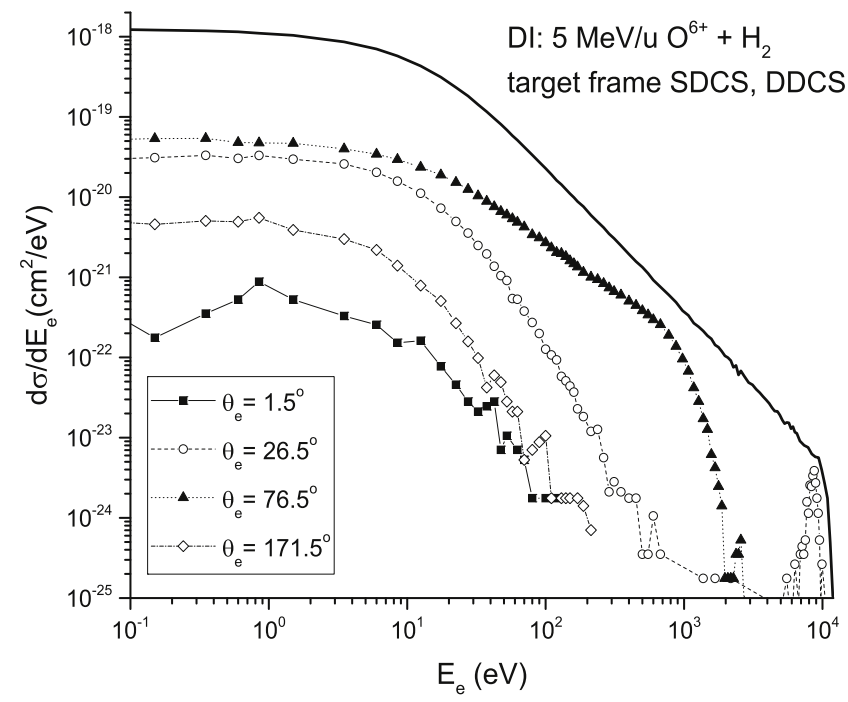

Fig. A.4. The doubly differential cross section for double ionization (DI) in $5 \mathrm{MeV} / \mathrm{u}$ $\mathrm{O}^{6+}+\mathrm{H}_{2}$ (curves with symbols for four electron ejection angles as indicated, as a function of ejected electron energy). The solid curve shows the singly differential cross section as a function of electron ejection angle, that is, the doubly differential cross section summed over electron ejection angles, for comparison.

\section{A.3. Double ionization}

Fig. A.4 shows an example of the DDCS for DI in $5 \mathrm{MeV} / \mathrm{u}$ $\mathrm{O}^{6+}$ impact. Similar behavior is seen to that of SI with both the increasing then decreasing magnitude with increasing angle and the variation at high electron-ejection energies associated with the binary peak.

\section{A.4. Single stripping}

We illustrate the behavior of the DDCS for SS in Figs. A.5 and A. 6 for $50 \mathrm{keV} / \mathrm{u} \mathrm{O}$ impact and $10 \mathrm{MeV} / \mathrm{u} \mathrm{O}^{6+}$ impact, respectively. For stripping, the ejected electrons, emitted in the projectile frame, are boosted by the projectile velocity in the target frame and 


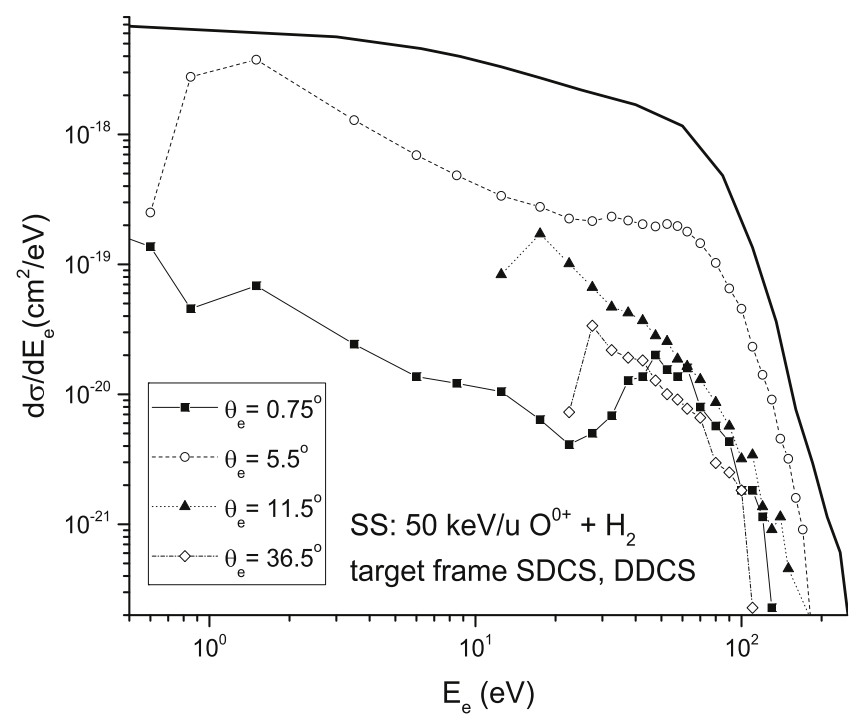

Fig. A.5. The doubly differential cross section for single stripping (SS) in $50 \mathrm{keV} / \mathrm{u}$ $\mathrm{O}+\mathrm{H}_{2}$ (curves with symbols for four electron ejection angles as indicated, as a function of ejected electron energy). The solid curve shows the singly differential cross section as a function of electron ejection angle, that is, the doubly differential cross section summed over electron ejection angles, for comparison.

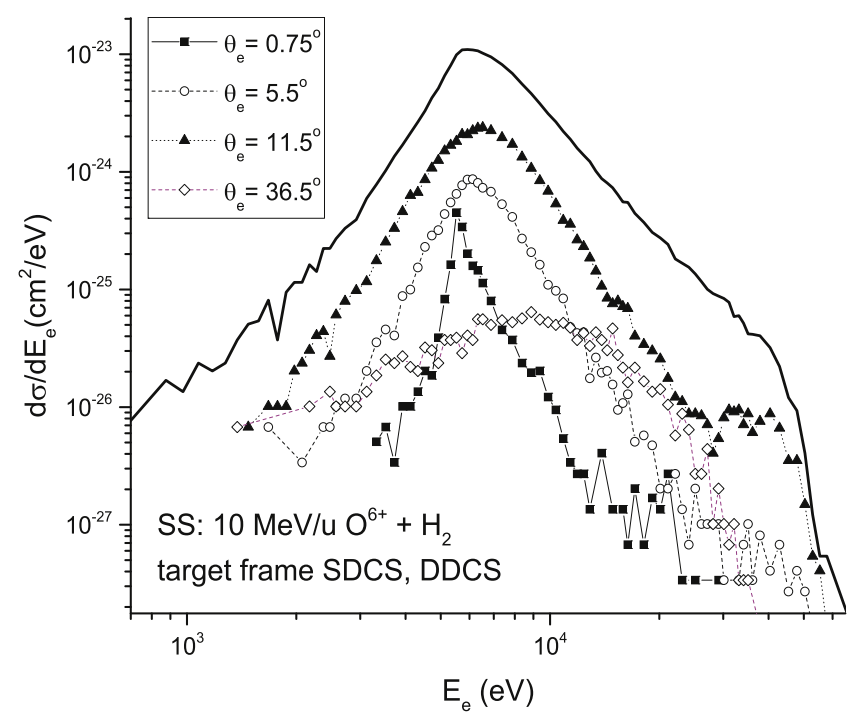

Fig. A.6. The doubly differential cross section for single stripping (SS) in $10 \mathrm{MeV} / \mathrm{u}$ $\mathrm{O}^{6+}+\mathrm{H}_{2}$ (curves with symbols for four electron ejection angles as indicated, as a function of ejected electron energy). The solid curve shows the singly differential cross section as a function of electron ejection angle, that is, the doubly differential cross section summed over electron ejection angles, for comparison.

therefore display somewhat different behavior, particularly for large projectile velocities, compared to emission from the target. For example, in SS of $\mathrm{O}$ in $50 \mathrm{keV} / \mathrm{u}$ impact, the DDCS curves have roughly the same shape for high-energy election-emission, but drop dramatically for lower electron-energy emission at large angles. In particular, in Fig. A.5, for 47.5 and 75 degrees, the lack of data points on the curves for less than about $20 \mathrm{eV}$ indicates that zero Monte Carlo events exist for those energies in the CTMC calculations.

For $10 \mathrm{MeV} / \mathrm{u} \mathrm{O}^{6+}$ impact, the curves show a peak roughly around the ejected-electron energy corresponding to emission in the projectile frame boosted by the high projectile velocity, about

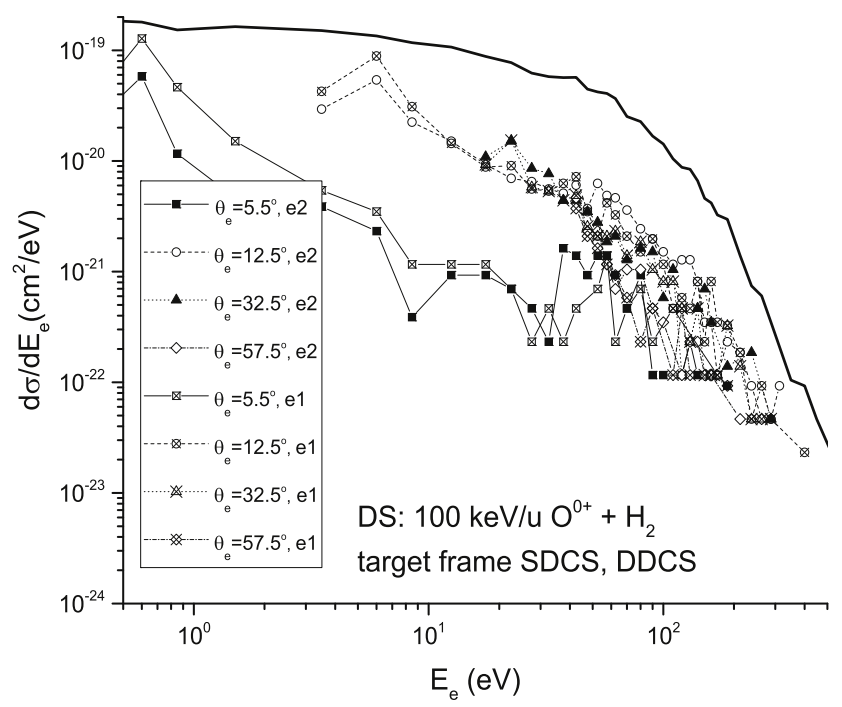

Fig. A.7. The doubly differential cross section for double stripping (DS) in $100 \mathrm{keV} / \mathrm{u}$ $\mathrm{O}+\mathrm{H}_{2}$ (curves with symbols for four electron ejection angles as indicated, and for electrons bound by the first ionization potential, "e1", and the second ionization potential, "e2", as a function of ejected electron energy). The solid curve shows the singly differential cross section as a function of electron ejection angle, that is, the doubly differential cross section summed over electron ejection angles, for comparison.

$5 \mathrm{keV}$. Particularly for small or relatively large emission angles, this peak shifts to lower or higher energies, respectively, and drops in magnitude compared to the emission at intermediate angles.

\section{A.5. Double stripping}

DS has an additional kind of energy-angle correlation because the energy transfer required to remove the two electrons is different, reflecting the sequential binding energies they possess. Consequently, there is a shift in cross section magnitude downward for the more deeply bound of the two electrons that are removed. This is illustrated in Fig. A.7 for $100 \mathrm{keV} / \mathrm{u} \mathrm{O}^{6+}+\mathrm{H}_{2}$. Also of note for this case is the cutting off at higher and higher ejected-electron energy of the curves (indicating dramatically dropping cross sections) for increasing electron-emission angle.

For collisions with small DS integral cross sections, too few CTMC DS events occur to allow this partitioning and so for $5 \mathrm{MeV} / \mathrm{u}$ $\mathrm{O}^{5+}+\mathrm{H}_{2}$, the DDCS displayed (see Fig. A.8) is summed over the two electrons emitted (as are all SDCS displayed and tabulated in this work). In addition, owing to the small number of counts and resulting large statistical variations in the DDCS, for this case only we have smoothed the curves for clarity of illustration. Behavior roughly the same as shown for SS at high projectile velocity (i.e., as shown in Fig. A.6) is evident.

\section{Appendix B. Transformation of ejected electron energies from the target to projectile frame}

As described in the text, and previously [1], the energy loss suffered by the projectile in any of the inelastic collision processes yielding a secondary electron can be modeled using the formulas given in Table A based on the average ejected electron energy (and other parameters such as the appropriate ionization potentials), or by sampling the SDCSs for the electron energy and angle. The SDCSs tabulated here and in Ref. [1] are given in the target frame, the frame in which secondary-electron simulations track their emission. 


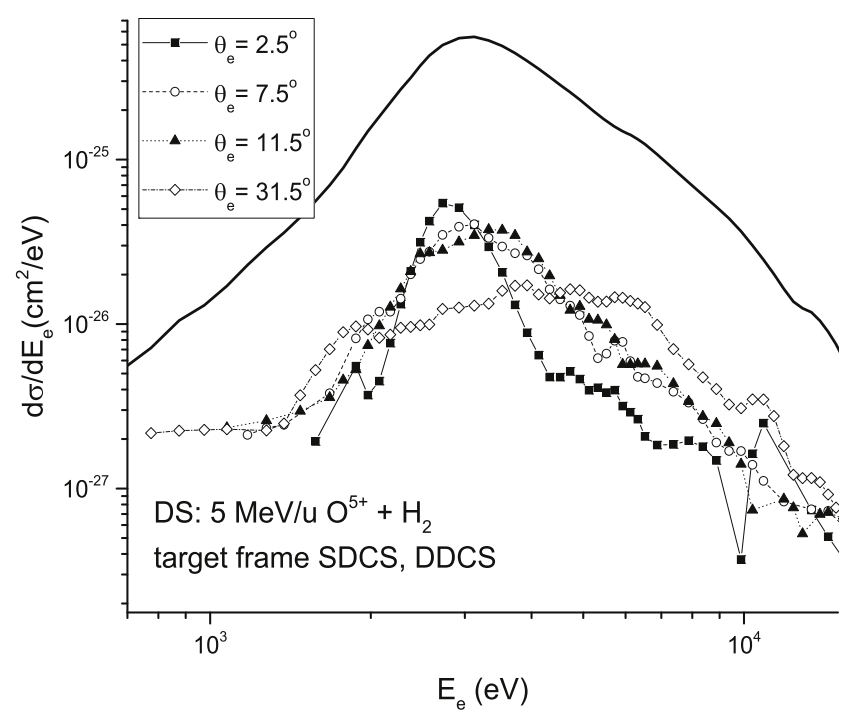

Fig. A.8. The doubly differential cross section for double stripping (DS) in $5 \mathrm{MeV} / \mathrm{u}$ $\mathrm{O}^{5+}+\mathrm{H}_{2}$ (curves with symbols for four electron ejection angles as indicated, as a function of ejected electron energy). The solid curve shows the singly differential cross section as a function of electron ejection angle, that is, the doubly differential cross section summed over electron ejection angles, for comparison. In this figure only, the four doubly differential cross sections curves have been smoothed for clarity of illustration owing to the very large Monte Carlo variations that they otherwise display.

For the target processes (SI, DI, TI, DCAI) the energy loss formulas use either the tabulated average energy loss based on the ejection of the electron in this frame or the electron energy sampled from the SDCSs. However, for the projectile processes (SS, DS), the appropriate average electron energy or the sampled electron energy is in the projectile frame. Consequently, simulations using sampling of the SDCSs must transform the target-frame electron energy for SS or DS into the projectile frame in order to use it in the energy loss formulas for these processes.

This may be accomplished by deriving expressions based on the calculated target-frame electron ejection energy and angle coming from emission boosted by the projectile velocity $\left(\mathrm{v}_{\text {proj }}\right)$. We begin by noting that the electron energy in the target frame, $\mathrm{E}_{e}^{\prime}$, that is, emitted in the projectile frame and boosted along the direction of travel of the projectile, $\hat{z}$, into the target frame, is given by

$\mathrm{E}_{e}^{\prime}=\frac{1}{2}\left[v_{x}^{2}+v_{y}^{2}+\left(v_{z}+v_{p r o j}\right)^{2}\right]$

where $v_{x}, v_{y}$ and $v_{z}$ are the components of the electron's velocity in the projectile frame. Similarly, the ejected electron angle in the projectile frame, $\theta_{e}^{\prime}$, is given by

$\theta_{e}^{\prime}=\cos ^{-1} \frac{v_{z}+v_{\text {proj }}}{\left[v_{x}^{2}+v_{y}^{2}+\left(v_{z}+v_{p r o j}^{2}\right)\right]^{\frac{1}{2}}}$.

Solving both these equations for $v^{2}\left(=v_{x}^{2}+v_{y}^{2}+v_{z}^{2}\right)$, the square of the ejected electron's velocity in the projectile frame, we obtain

$v^{2}=2 \mathrm{E}_{e}^{\prime}-2 v_{z} v_{p r o j}-v_{p r o j}^{2}$

$v^{2}=\frac{\left(v_{z}+v_{\text {proj }}\right)^{2}}{\cos ^{2} \theta_{e}^{\prime}}-2 v_{z} v_{\text {proj }}-v_{\text {proj }}^{2}$.

Inspection of these two equations allows us to equate like terms and identify an expression solvable for $v_{z}$ in terms of only known quantities, $\mathrm{E}_{e}^{\prime}$ from the sampling of the SDCS as a function of ejected electron energy, $\theta_{e}^{\prime}$ from the sampling of the SDCS as a function of ejected electron angle, and $v_{\text {proj }}$ from the relevant impact energy considered:

$2 \mathrm{E}_{e}^{\prime}=\frac{\left(v_{z}+v_{\text {proj }}\right)^{2}}{\cos ^{2} \theta_{e}^{\prime}}$

from which we derive

$v_{z}=\sqrt{2 \mathrm{E}_{e}^{\prime}} \cos \theta_{e}^{\prime}-v_{\text {proj }}$.

Therefore, given sampled values of $\mathrm{E}_{e}^{\prime}$ and $\theta_{e}^{\prime}$, Eq. (B.6) may be used to obtain $v_{z}$ and from it the value of the projectile-frame electron energy $\mathrm{E}_{e}$ using $\mathrm{E}_{e}=\frac{1}{2} m_{e} v^{2}$ with $v^{2}$ from Eq. (B.3). While not generally needed in simulations, we note that the projectile frame electron ejection angle, $\theta_{e}$, is found from the expression $\theta_{e}=\cos ^{-1} v_{z} / \sqrt{v^{2}}$.

Consider as an example, sampling of the SDCSs for SS in 50 $\mathrm{keV} / \mathrm{u} \mathrm{O}^{3+}+\mathrm{H}_{2}$ which might yield values in the target frame of $\mathrm{E}_{e}^{\prime}=92.71 \mathrm{eV}$ and $\theta_{e}^{\prime}=9.116 \mathrm{deg}$. Converting energy in eV to atomic units ( 1 a.u. $=27.2116 \mathrm{eV}$ ), Eq. (B.6) yields $v_{z}=1.162$ a.u., and from equation Eq. (B.3), the projectile frame ejected electron energy $E_{e}=0.7614$ a.u. $=20.72 \mathrm{eV}$, and projectile frame ejected electron angle $\theta_{e}=19.58 \mathrm{deg}$. Using Table $\mathrm{A}$, the energy loss associated with this SS event is, therefore, $\Delta e^{\mathrm{SS}}=20.72 \mathrm{eV}+77.41$ $\mathrm{eV}=98.13 \mathrm{eV}$, where the ionization potential of $\mathrm{O}^{3+}$ is $77.41 \mathrm{eV}$. Inspection of Table 1, which tabulates the average energy loss for SS in $50 \mathrm{keV} / \mathrm{u} \mathrm{O}^{3+}+\mathrm{H}_{2}$, we find a value of $105 \mathrm{eV}$, not far from the result of this example.

We note that sampling of the SDCSs to obtain the ejectedelectron energy and angle for SS and DS in the target frame separately, and then transforming to the projectile frame, misses some degree of the electron energy-angle correlation (see Appendix A) in the resulting event-by-event projectile-frame energies, and thus energy loss. Fortunately the magnitude of this difference is very small for low impact energies, rising to about $1-5 \%$ at $75 \mathrm{keV} / \mathrm{u}$ and to between 5 and $20 \%$ for $25 \mathrm{MeV} / \mathrm{u}$ (the range given here reflecting not only the variation in the degree of energy-angle correlation but the Monte Carlo statistical variation). For the target-frame electron-emission processes (i.e., SI, TI, DI) sampling the SDCS as a function of electron energy to obtain the energy loss does not as strongly reflect this effect since without the need for the sampling of the SDCS as a function of angle and performing the frame transformation it is already integrated over ejection angles.

\section{References}

[1] D.R. Schultz, N. Ozak, T.E. Cravens, H. Gharibnejad, At. Data Nucl. Data Tables 113 (2017) 1.

[2] N. Ozak, T.E. Cravens, D.R. Schultz, Geophys. Res. Lett. 40 (2013) 4144

[3] S.J. Houston, N. Ozak, J. Young, T.E. Cravens, D.R. Schultz, J. Geophys. Res. Space Phys. 123 (2018).

[4] Y.-W. Hui, D.R. Schultz, V.A. Kharchenko, P.C. Stancil, T.E. Cravens, C.M. Lisse, A. Dalgarno, Astrophys. J. Lett. 702 (2009) L158.

[5] N. Ozak, D.R. Schultz, T.E. Cravens, V. Kharchenko, Y.-W. Hui, J. Geophys. Res. 115, A11 (2010) 306.

[6] Y.-W. Hui, D.R. Schultz, V.A. Kharchenko, A. Bhardwaj, G. Branduardi-Raymont, P.C. Stancil, T.E. Cravens, C.M. Lisse, A. Dalgarno, J. Geophys. Res. 115 (2010) A07102.

[7] M. Horanyi, T.E. Cravens, J.H. Waite, J. Geophys. Res. 93 (1988) 7251.

[8] T.E. Cravens, E. Howell, J.H. Waite, G.R. Gladstone, J. Geophys. Res. 100 (1995) 17153.

[9] V. Kharchenko, W. Liu, A. Dalgarno, J. Geophys. Res. 103 (1998) 26687.

[10] V. Kharchenko, A. Dalgarno, D.R. Schultz, P.C. Stancil, Geophys. Res. Lett. 33 (2006) 11105.

[11] V. Kharchenko, A. Bhardwaj, A. Dalgarno, D.R. Schultz, P.C. Stancil, J. Geophys. Res. 133 (2008) 8229.

[12] W. Liu, D.R. Schultz, Astrophys. J. 526 (1999) 538

[13] T.E. Cravens, J.H. Waite, T.I. Gombosi, N. Lugaz, G.R. Gladstone, B.H. Mauk, R.J. MacDowall, J. Geophys. Res. 108 (2003) 1465.

[14] R. Abrines, I.C. Percival, Proc. Phys. Soc. 88 (1966) 861.

[15] R.E. Olson, A. Salop, Phys. Rev. A 16 (1977) 531.

[16] L. Meng, C.O. Reinhold, R.E. Olson, Phys. Rev. A 40 (1989) 3637.

[17] L. Meng, C.O. Reinhold, R.E. Olson, Phys. Rev. A 42 (1990) 5286. 
[18] R.E. Olson, J. Ullrich, H. Schmidt-Böcking, Phys. Rev. A 39 (1989) 5572.

[19] G.W. Kerby, M.W. Gealy, Y.-Y. Hsu, M.E. Rudd, D.R. Schultz, C.O. Reinhold, Phys Rev. A 51 (1995) 2256

[20] Y.-Y.Hsu, G.W. Kerby, M.W. Gealy, M.E. Rudd, D.R. Schultz, C.O. Reinhold, Phys. Rev. A 53 (1995) 303.

[21] C.O. Reinhold, D.R. Schultz, R.E. Olson, Nucl. Instrum. Methods B 56/57 (1991) 271.

[22] L.H. Toburen, R.D. DuBois, C.O. Reinhold, D.R. Schultz, R.E. Olson, Phys. Rev. A $42(1990) 5338$

[23] D.R. Schultz, C.O. Reinhold, Phys. Rev. A 50 (1994) 2390.

[24] M. Sataka, M. Imai, Y. Yamazaki, K. Komaki, K. Kawatsura, Y. Kanai, H. Tawara, D.R. Schultz, C.O. Reinhold, J. Phys. B 27 (1994) L171.

[25] L.H. Toburen, S.L. McLawhorn, R.S. McLawhorn, N. Evans, E.L.B. Justiniano, J.L. Shinpaugh, D.R. Schultz, C.O. Reinhold, Radiat. Prot. Dosim. 122 (2006) 22.

[26] D.R. Schultz, P.C. Stancil, M.J. Rakovic, J. Phys. B 34 (2001) 2739.

[27] D.R. Schultz, R.E. Olson, C.O. Reinhold, S. Kelbch, C. Kelbch, H. Schmidt-Böcking, J. Ullrich, J. Phys. B 23 (1990) 3839.
[28] C.O. Reinhold, J. Burgdörfer, J. Phys. B 26 (1993) 3101.

[29] Stopping and Range of Ions in Matter, version 2013.00, 2013.

[30] G. Schiwietz, P.L. Grande, Nucl. Instrum. Methods Phys. Res. B 175 (2001) 125.

[31] I.S. Dmitriev, V.S. Nikoleav, Zh. Eksperim. I Teor. Fiz 47 (1964) 615; Sov. Phys.JETP 20 (1965) 409.

[32] J. Østgaard Olsen, P. Hvelplund, J. Phys. B 7 (1974) 1331.

[33] T. Jorgensen, C.E. Kuyatt, W.W. Lang, D.C. Lorents, C.A. Sautter, Phys. Rev. A 140 (1965) 1481.

[34] B.G. Lindsay, W.S. Yu, K.F. McDonald, R.F. Stebbings, Phys. Rev. A 70 (2004) 042701.

[35] Ia.M. Fogel, V.A. Ankudinov, D.V. Pilipenko, Sov. Phys.-JETP 35 (1959) 601

[36] P. Hvelplund, Danske Videnskab. Selskab Mat.-Fys. Medd. 38 (1971) 4.

[37] J. Herault, R. Bimbot, H. Gauvin, B. Kubica, R. Anne, G. Bastin, R. Hubert, Nucl. Instrum. Methods Phys. Res. B 61 (1991) 156.

[38] R. Bimbot, H. Gauvin, J. Herault, R. Anne, G. Bastin, R. Hubert, Radiat. Eff. Defects Solids 110 (1989) 15 


\section{Explanation of Tables}

Table 1.

Table 2.

Table 3.

Table 4.

Table 5.

Table 6.

Table 7.

Table 8.

Table 9.

Table 10.

Table 11.

Tables 12-14.

Tables 15-17.

Tables 18-20.

Tables 21-23.

Tables 24-26.

Tables 27-29.

Tables 30-32

The integral cross section and associated average energy loss for single and double stripping for $0^{q+}+\mathrm{H}_{2}(q=0-8)$ collisions with impact energies between 1 and $25000 \mathrm{keV} / \mathrm{u}$.

For each collision energy, $\mathrm{E}$ in $\mathrm{keV} / \mathrm{u}$, the columns list the integral cross section in $\mathrm{cm}^{2}$ and then the average energy loss in eV for each ionization stage. Most cross sections and energy losses reflect results of larger Monte Carlo runs compared to those of Ref. [1]. Footnotes indicate results from extrapolations, cross sections that were improved significantly (e.g., 25 percent or more), or that correct a typographical error in the previous work

The integral cross section and associated average energy loss for single and double projectile excitation for $\mathrm{O}^{q+}+\mathrm{H}_{2}(q=0-8)$ collisions with impact energies between 1 and $25000 \mathrm{keV} / \mathrm{u}$.

Same as for Table 1.

The integral cross section and associated average energy loss for NSIM and SIM processes in $\mathrm{O}+\mathrm{H}_{2}$ collisions with impact energies between 1 and $25000 \mathrm{keV} / \mathrm{u}$.

For each collision energy, $\mathrm{E}$ in $\mathrm{keV} / \mathrm{u}$, the columns list the integral cross section in $\mathrm{cm}^{2}$ and then the average energy loss in eV for each NSIM and SIM process. Footnotes indicate results from extrapolations, cross sections that were improved significantly (e.g., 25 percent or more), or that correct a typographical error in the previous work.

The integral cross section and associated average energy loss for NSIM and SIM processes in $\mathrm{O}^{+}+\mathrm{H}_{2}$ collisions with impact energies between 1 and $25000 \mathrm{keV} / \mathrm{u}$.

Same as for Table 3.

The integral cross section and associated average energy loss for NSIM and SIM processes in $\mathrm{O}^{2+}+\mathrm{H}_{2}$ collisions with impact energies between 1 and $25000 \mathrm{keV} / \mathrm{u}$.

Same as for Table 3. In addition the asterisks indicate the entries that include the low-energy electron correction from the first Born approximation as described in the text.

The integral cross section and associated average energy loss for NSIM and SIM processes in $\mathrm{O}^{3+}+\mathrm{H}_{2}$ collisions with impact energies between 1 and $25000 \mathrm{keV} / \mathrm{u}$.

Same as for Table 5 .

The integral cross section and associated average energy loss for NSIM and SIM processes in $\mathbf{0}^{4+}+\mathrm{H}_{2}$ collisions with impact energies between 1 and $25000 \mathrm{keV} / \mathrm{u}$.

Same as for Table 5 .

The integral cross section and associated average energy loss for NSIM and SIM processes in $\mathbf{0}^{5+}+\mathrm{H}_{2}$ collisions with impact energies between 1 and $25000 \mathrm{keV} / \mathrm{u}$.

Same as for Table 5 .

The integral cross section and associated average energy loss for NSIM and SIM processes in $\mathbf{0}^{6+}+\mathrm{H}_{2}$ collisions with impact energies between 1 and $25000 \mathrm{keV} / \mathrm{u}$.

Same as for Table 5 .

The integral cross section and associated average energy loss for NSIM and SIM processes in $\mathbf{0}^{7+}+\mathrm{H}_{2}$ collisions with impact energies between 1 and $25000 \mathrm{keV} / \mathrm{u}$.

Same as for Table 5 .

The integral cross section and associated average energy loss for NSIM processes in $\mathbf{0}^{8+}+\mathrm{H}_{2}$ collisions with impact energies between 1 and $25000 \mathrm{keV} / \mathrm{u}$.

Same as for Table

The singly differential cross section as a function of the ejected electron energy for single ionization in $5000-25000 \mathrm{keV} / \mathrm{u} 0^{q+}$ $(q=0-8)+\mathbf{H}_{2}$ collisions.

Cross sections are given in $\mathrm{cm}^{2} / \mathrm{eV}$.

The singly differential cross section as a function of the ejected electron angle for single ionization in 5000-25 000 keV/u $0 \mathrm{q}+(q=0-8)$ $+\mathbf{H}_{2}$ collisions.

Cross sections are given in $\mathrm{cm}^{2} / \mathrm{sr}$.

The singly differential cross section as a function of the ejected electron energy for double ionization in $5000-25000 \mathrm{keV} / \mathrm{u} 00^{q+}$ $(q=0-8)+H_{2}$ collisions.

Cross sections are given in $\mathrm{cm}^{2} / \mathrm{eV}$.

The singly differential cross section as a function of the ejected electron angle for double ionization in $5000-25000 \mathrm{keV} / \mathrm{u}^{\mathrm{q}+}$ $(q=0-8)+\mathbf{H}_{2}$ collisions.

Cross sections are given in $\mathrm{cm}^{2} / \mathrm{sr}$.

The singly differential cross section as a function of the ejected electron energy for transfer ionization in $5000-25000 \mathrm{keV} / \mathrm{u} 00^{q+}$ $(q=\mathbf{0}-8)+\mathbf{H}_{2}$ collisions. Cross sections are given in $\mathrm{cm}^{2} / \mathrm{eV}$

The singly differential cross section as a function of the ejected electron angle for transfer ionization in $5000-25000 \mathrm{keV} / \mathrm{u} 0^{\mathrm{q}+}$ $(q=0-8)+\mathrm{H}_{2}$ collisions.

Cross sections are given in $\mathrm{cm}^{2} / \mathrm{sr}$

The singly differential cross section as a function of the ejected electron energy for single stripping in $5000-25000 \mathrm{keV} / \mathrm{u}^{\mathrm{q}+}$

$(q=0-7)+\mathbf{H}_{2}$ collisions.

Cross sections are given in $\mathrm{cm}^{2} / \mathrm{eV}$ 
Tables 33-35.

Tables 36-38.

Tables 39-41.
The singly differential cross section as a function of the ejected electron angle for single stripping in 5000-25 000 keV/u 0 q+ $(q=0-7)$ $+\mathrm{H}_{2}$ collisions.

Cross sections are given in $\mathrm{cm}^{2} / \mathrm{sr}$.

The singly differential cross section as a function of the ejected electron energy for double stripping in 5000-25 000 keV/u $0^{q+}$ $(q=0-6)+H_{2}$ collisions.

Cross sections are given in $\mathrm{cm}^{2} / \mathrm{eV}$.

The singly differential cross section as a function of the ejected electron angle for double stripping in 5000-25 000 keV/u 0 ${ }^{q+}(q=0-6)$ $+\mathrm{H}_{2}$ collisions.

Cross sections are given in $\mathrm{cm}^{2} / \mathrm{sr}$. 
Table 1

The integral cross section and associated average energy loss for single and double stripping for $\mathrm{O}^{q+}+\mathrm{H}_{2}(q=0-8)$ collisions with impact energies between 1 and 25000 $\mathrm{keV} / \mathrm{u}$.

\begin{tabular}{|c|c|c|c|c|c|c|c|c|}
\hline \multicolumn{9}{|c|}{ Single Stripping } \\
\hline \multirow{3}{*}{$\begin{array}{l}\mathrm{E} \\
\mathrm{keV} / \mathrm{u}\end{array}$} & \multirow{3}{*}{$\begin{array}{l}\mathrm{O} \\
\text { cross section } \\
\mathrm{cm}^{2}\end{array}$} & \multirow{3}{*}{$\begin{array}{l}\text { Eloss } \\
\mathrm{eV}\end{array}$} & \multicolumn{2}{|l|}{$\mathrm{O}^{+}$} & \multicolumn{2}{|l|}{$\mathrm{O}^{2+}$} & \multicolumn{2}{|l|}{$\mathrm{O}^{3+}$} \\
\hline & & & cross section & Eloss & cross section & Eloss & cross section & Eloss \\
\hline & & & $\mathrm{cm}^{2}$ & $\mathrm{eV}$ & $\mathrm{cm}^{2}$ & $\mathrm{eV}$ & $\mathrm{cm}^{2}$ & $\mathrm{eV}$ \\
\hline 1 & $3.41 \mathrm{E}-17$ & 21.1 & $1.91 \mathrm{E}-18$ & 46.9 & $2.32 \mathrm{E}-19$ & 68.4 & $1.43 \mathrm{E}-20$ & 92.2 \\
\hline 10 & $1.89 \mathrm{E}-16$ & 24.0 & $3.39 \mathrm{E}-17$ & 46.1 & $5.62 \mathrm{E}-18$ & 67.2 & $7.98 \mathrm{E}-19$ & 91.6 \\
\hline 50 & $1.79 \mathrm{E}-16$ & 44.9 & $7.82 \mathrm{E}-17$ & 65.2 & $3.71 \mathrm{E}-17$ & 82.3 & $1.29 \mathrm{E}-17$ & 105. \\
\hline 75 & $1.64 \mathrm{E}-16$ & 50.5 & $7.59 \mathrm{E}-17$ & 75.0 & $4.10 \mathrm{E}-17$ & 93.3 & $1.86 \mathrm{E}-17$ & 114. \\
\hline 100 & $1.52 \mathrm{E}-16$ & 53.8 & $7.09 \mathrm{E}-17$ & 83.0 & $4.09 \mathrm{E}-17$ & 102. & $2.07 \mathrm{E}-17$ & 123. \\
\hline 200 & $1.23 \mathrm{E}-16$ & 58.8 & $5.64 \mathrm{E}-17$ & 100. & $3.30 \mathrm{E}-17$ & 128. & $1.85 \mathrm{E}-17$ & 154. \\
\hline 500 & $8.48 \mathrm{E}-17$ & 60.7 & $3.56 \mathrm{E}-17$ & 116. & $2.02 \mathrm{E}-17$ & 159. & $1.12 \mathrm{E}-17$ & 199. \\
\hline 1000 & $5.99 \mathrm{E}-17$ & 60.5 & $2.30 \mathrm{E}-17$ & 126. & $1.23 \mathrm{E}-17$ & 177. & $6.66 \mathrm{E}-18$ & 231. \\
\hline 2000 & $3.99 \mathrm{E}-17$ & 59.0 & $1.41 \mathrm{E}-17$ & 131. & $7.12 \mathrm{E}-18$ & 189. & $\ddagger 3.75 \mathrm{E}-18$ & 259. \\
\hline 5000 & $2.14 \mathrm{E}-17$ & 57.8 & $6.75 \mathrm{E}-18$ & 138. & $3.24 \mathrm{E}-18$ & 209. & $1.64 \mathrm{E}-18$ & 286. \\
\hline 10000 & $1.27 \mathrm{E}-17$ & 58.6 & $3.70 \mathrm{E}-18$ & 143. & $1.72 \mathrm{E}-18$ & 216. & $6.74 \mathrm{E}-19$ & 321. \\
\hline \multirow[t]{2}{*}{25000} & $5.94 \mathrm{E}-18$ & 58.0 & $1.61 \mathrm{E}-18$ & 145. & $7.25 \mathrm{E}-19$ & 245 & $3.15 \mathrm{E}-19$ & 346. \\
\hline & \multicolumn{2}{|l|}{$\mathrm{O}^{4+}$} & \multicolumn{2}{|l|}{$\mathrm{O}^{5+}$} & \multicolumn{2}{|l|}{$\mathrm{O}^{6+}$} & \multicolumn{2}{|l|}{$\mathrm{O}^{7+}$} \\
\hline E & cross section & Eloss & cross section & Eloss & cross section & Eloss & cross section & Eloss \\
\hline $\mathrm{keV} / \mathrm{u}$ & $\mathrm{cm}^{2}$ & $\mathrm{eV}$ & $\mathrm{cm}^{2}$ & $\mathrm{eV}$ & $\mathrm{cm}^{2}$ & $\mathrm{eV}$ & $\mathrm{cm}^{2}$ & $\mathrm{eV}$ \\
\hline 1 & $2.53 \mathrm{E}-22$ & 133. & $1.76 \mathrm{E}-24$ & 149. & $\dagger 1.00 \mathrm{E}-30$ & 829. & $\dagger 1.00 \mathrm{E}-30$ & 971. \\
\hline 10 & $4.71 \mathrm{E}-20$ & 131. & $5.54 \mathrm{E}-21$ & 160. & $\dagger 1.00 \mathrm{E}-25$ & 839. & $\dagger 5.00 \mathrm{E}-26$ & 991. \\
\hline 50 & $2.17 \mathrm{E}-18$ & 145. & $5.19 \mathrm{E}-19$ & 172. & $1.01 \mathrm{E}-22$ & 858. & $2.09 \mathrm{E}-23$ & 1011. \\
\hline 75 & $4.35 \mathrm{E}-18$ & 153. & $1.17 \mathrm{E}-18$ & 179. & $5.03 \mathrm{E}-22$ & 864. & $9.54 \mathrm{E}-23$ & 1019. \\
\hline 100 & $6.06 \mathrm{E}-18$ & 159. & $1.82 \mathrm{E}-18$ & 185. & $1.26 \mathrm{E}-21$ & 897. & $2.76 \mathrm{E}-22$ & 1045. \\
\hline 200 & $7.54 \mathrm{E}-18$ & 193. & $2.87 \mathrm{E}-18$ & 218 & $9.59 \mathrm{E}-21$ & 915. & $2.06 \mathrm{E}-21$ & 1080. \\
\hline 500 & $5.02 \mathrm{E}-18$ & 257. & $2.07 \mathrm{E}-18$ & 293. & $9.19 \mathrm{E}-20$ & 955. & $2.58 \mathrm{E}-20$ & 1103. \\
\hline 1000 & $3.00 \mathrm{E}-18$ & 303. & $1.23 \mathrm{E}-18$ & 354. & $1.97 \mathrm{E}-19$ & 1084. & $6.83 \mathrm{E}-20$ & 1227. \\
\hline 2000 & $1.69 \mathrm{E}-18$ & 350. & $6.65 \mathrm{E}-19$ & 408. & $1.87 \mathrm{E}-19$ & 1339. & $7.48 \mathrm{E}-20$ & 1490. \\
\hline 5000 & $7.15 \mathrm{E}-19$ & 399. & $2.85 \mathrm{E}-19$ & 470 & $9.86 \mathrm{E}-20$ & 1780. & $4.09 \mathrm{E}-20$ & 2018. \\
\hline 10000 & $3.68 \mathrm{E}-19$ & 444. & $1.45 \mathrm{E}-19$ & 503. & $5.28 \mathrm{E}-20$ & 2135. & $2.19 \mathrm{E}-20$ & 2377. \\
\hline 25000 & $1.51 \mathrm{E}-19$ & 531. & $5.93 \mathrm{E}-20$ & 552. & $2.04 \mathrm{E}-20$ & 2496. & $9.00 \mathrm{E}-21$ & 2698. \\
\hline \multicolumn{9}{|c|}{ Double Stripping } \\
\hline & 0 & & $\mathrm{O}^{+}$ & & $\mathrm{O}^{2+}$ & & $\mathrm{O}^{3+}$ & \\
\hline $\mathrm{E}$ & cross section & & cross section & Eloss & cross section & Eloss & cross section & Eloss \\
\hline $\mathrm{keV} / \mathrm{u}$ & $\mathrm{cm}^{2}$ & $\mathrm{eV}$ & $\mathrm{cm}^{2}$ & $\mathrm{eV}$ & $\mathrm{cm}^{2}$ & $\mathrm{eV}$ & $\mathrm{cm}^{2}$ & $\mathrm{eV}$ \\
\hline 1 & $1.23 \mathrm{E}-20$ & 60.7 & $8.62 \mathrm{E}-23$ & 107. & $\dagger 1.00 \mathrm{E}-24$ & 142. & $\dagger 1.00 \mathrm{E}-24$ & 201. \\
\hline 10 & $1.85 \mathrm{E}-18$ & 75.3 & $8.37 \mathrm{E}-20$ & 116. & $5.08 \mathrm{E}-21$ & 161. & $\dagger 1.00 \mathrm{E}-21$ & 221. \\
\hline 50 & $5.62 \mathrm{E}-18$ & 110. & $1.56 \mathrm{E}-18$ & 151. & $3.76 \mathrm{E}-19$ & 192. & $5.36 \mathrm{E}-20$ & 263. \\
\hline 75 & $6.36 \mathrm{E}-18$ & 127. & $1.95 \mathrm{E}-18$ & 172. & $6.39 \mathrm{E}-19$ & 213. & $1.58 \mathrm{E}-19$ & 275. \\
\hline 100 & $6.19 \mathrm{E}-18$ & 139. & $2.12 \mathrm{E}-18$ & 189. & $8.52 \mathrm{E}-19$ & 228 & $2.95 \mathrm{E}-19$ & 291. \\
\hline 200 & $5.09 \mathrm{E}-18$ & 175. & $1.80 \mathrm{E}-18$ & 230 & $8.93 \mathrm{E}-19$ & 284. & $4.42 \mathrm{E}-19$ & 352. \\
\hline 500 & $2.72 \mathrm{E}-18$ & 219. & $8.97 \mathrm{E}-19$ & 303. & $4.52 \mathrm{E}-19$ & 380. & $2.59 \mathrm{E}-19$ & 462. \\
\hline 1000 & $1.36 \mathrm{E}-18$ & 241. & $4.29 \mathrm{E}-19$ & 335. & $2.08 \mathrm{E}-19$ & 421. & $1.25 \mathrm{E}-19$ & 549. \\
\hline 2000 & $6.36 \mathrm{E}-19$ & 244. & $1.57 \mathrm{E}-19$ & 351. & $7.92 \mathrm{E}-20$ & 446. & $4.55 \mathrm{E}-20$ & 617. \\
\hline 5000 & $1.86 \mathrm{E}-19$ & 234. & $4.34 \mathrm{E}-20$ & 334. & $1.94 \mathrm{E}-20$ & 452. & $1.04 \mathrm{E}-20$ & 696. \\
\hline 10000 & $6.91 \mathrm{E}-20$ & 244. & $1.43 \mathrm{E}-20$ & 349. & $6.28 \mathrm{E}-21$ & 530. & $3.28 \mathrm{E}-21$ & 735. \\
\hline \multirow[t]{2}{*}{25000} & $1.68 \mathrm{E}-20$ & 243. & $3.08 \mathrm{E}-21$ & 384. & $1.22 \mathrm{E}-21$ & 551. & $6.27 \mathrm{E}-22$ & 864. \\
\hline & $\mathrm{O}^{4+}$ & & $\mathrm{O}^{5+}$ & & $\mathrm{O}^{6+}$ & & & \\
\hline $\mathrm{E}$ & cross section & Eloss & cross section & Eloss & cross section & Eloss & & \\
\hline $\mathrm{keV} / \mathrm{u}$ & $\mathrm{cm}^{2}$ & $\mathrm{eV}$ & $\mathrm{cm}^{2}$ & $\mathrm{eV}$ & $\mathrm{cm}^{2}$ & $\mathrm{eV}$ & & \\
\hline 1 & $\dagger 1.00 \mathrm{E}-25$ & 267. & $\dagger 1.00 \mathrm{E}-29$ & 897. & $\dagger 1.00 \mathrm{E}-30$ & 1691. & & \\
\hline 10 & $\dagger 1.00 \mathrm{E}-22$ & 292. & $\dagger 1.00 \mathrm{E}-26$ & 942. & $\dagger 1.00 \mathrm{E}-27$ & 1811. & & \\
\hline 50 & $6.31 \mathrm{E}-21$ & 334. & $+2.00 \mathrm{E}-24$ & 1027. & $\dagger 1.00 \mathrm{E}-25$ & 2011. & & \\
\hline 75 & $2.22 \mathrm{E}-20$ & 342. & $+1.00 \mathrm{E}-23$ & 1077. & $\dagger 5.00 \mathrm{E}-25$ & 2061. & & \\
\hline 100 & $4.79 \mathrm{E}-20$ & 352. & $3.30 \mathrm{E}-23$ & 1111. & $\ddagger 1.60 \mathrm{E}-24$ & 2109. & & \\
\hline 200 & $1.06 \mathrm{E}-19$ & 413. & $2.09 \mathrm{E}-22$ & 1214. & $\ddagger 3.70 \mathrm{E}-24$ & 2160. & & \\
\hline 500 & $6.75 \mathrm{E}-20$ & 543. & $2.55 \mathrm{E}-21$ & 1264. & $2.37 \mathrm{E}-22$ & 2134. & & \\
\hline 1000 & $2.92 \mathrm{E}-20$ & 637. & $5.01 \mathrm{E}-21$ & 1506. & $1.34 \mathrm{E}-21$ & 2318. & & \\
\hline 2000 & $1.07 \mathrm{E}-20$ & 720. & $5.71 \mathrm{E}-21$ & 1883. & $1.77 \mathrm{E}-21$ & 2894. & & \\
\hline 5000 & $2.29 \mathrm{E}-21$ & 823. & $1.81 \mathrm{E}-21$ & 2440. & $6.24 \mathrm{E}-22$ & 3481. & & \\
\hline 10000 & $6.99 \mathrm{E}-22$ & 912. & $6.18 \mathrm{E}-22$ & 2838. & $2.17 \mathrm{E}-22$ & 3972. & & \\
\hline 25000 & $1.34 \mathrm{E}-22$ & 1280. & $1.25 \mathrm{E}-22$ & 3344. & $4.22 \mathrm{E}-23$ & 5389. & & \\
\hline
\end{tabular}

$\dagger$ Extrapolated. $\quad \ddagger$ Significantly improved. 
Table 2

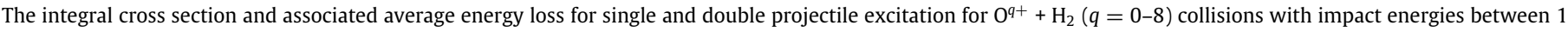
and $25000 \mathrm{keV} / \mathrm{u}$.

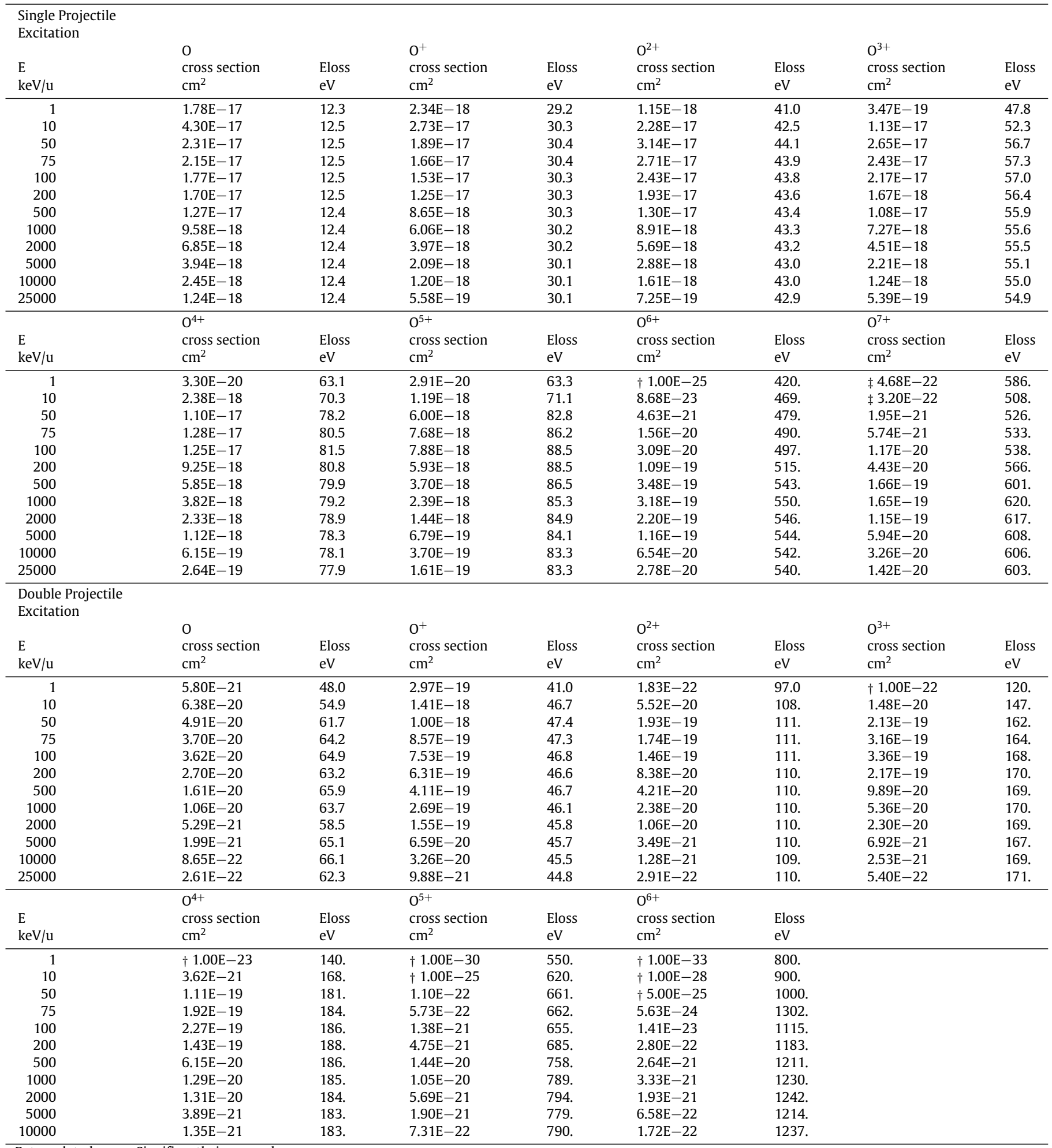

† Extrapolated. $\quad$ Significantly improved. 
Table 3

The integral cross section and associated average energy loss for NSIM and SIM processes in $\mathrm{O}+\mathrm{H}_{2}$ collisions with impact energies between 1 and 25000 keV/u.

\begin{tabular}{|c|c|c|c|c|c|c|c|c|c|c|c|c|}
\hline \multirow[t]{3}{*}{ Channel } & \multicolumn{12}{|l|}{ Energy } \\
\hline & \multicolumn{2}{|l|}{$1 \mathrm{keV} / \mathrm{u}$} & \multicolumn{2}{|l|}{10} & \multicolumn{2}{|l|}{50} & \multicolumn{2}{|l|}{75} & \multicolumn{2}{|l|}{100} & \multicolumn{2}{|l|}{200} \\
\hline & $\begin{array}{l}\text { cross } \\
\text { section } \\
\mathrm{cm}^{2}\end{array}$ & $\begin{array}{l}\text { Eloss } \\
\mathrm{eV}\end{array}$ & $\begin{array}{l}\text { cross } \\
\text { section } \\
\mathrm{cm}^{2}\end{array}$ & $\begin{array}{l}\text { Eloss } \\
\mathrm{eV}\end{array}$ & $\begin{array}{l}\text { cross } \\
\text { section } \\
\mathrm{cm}^{2}\end{array}$ & $\begin{array}{l}\text { Eloss } \\
\mathrm{eV}\end{array}$ & $\begin{array}{l}\text { cross } \\
\text { section } \\
\mathrm{cm}^{2}\end{array}$ & $\begin{array}{l}\text { Eloss } \\
\mathrm{eV}\end{array}$ & $\begin{array}{l}\text { cross } \\
\text { section } \\
\mathrm{cm}^{2}\end{array}$ & $\begin{array}{l}\text { Eloss } \\
\mathrm{eV}\end{array}$ & $\begin{array}{l}\text { cross } \\
\text { section } \\
\mathrm{cm}^{2}\end{array}$ & $\begin{array}{l}\text { Eloss } \\
\mathrm{eV}\end{array}$ \\
\hline SI & $1.07 \mathrm{E}-17$ & $\ddagger 30.4$ & $9.94 \mathrm{E}-19$ & 36.0 & $1.14 \mathrm{E}-17$ & 60.3 & $9.67 \mathrm{E}-18$ & 80.0 & $7.54 \mathrm{E}-18$ & 97.8 & $6.09 \mathrm{E}-18$ & 145. \\
\hline $\mathrm{SI}+\mathrm{SS}$ & $6.16 \mathrm{E}-18$ & 51.4 & $3.71 \mathrm{E}-17$ & 60.0 & $8.44 \mathrm{E}-17$ & 105. & $8.44 \mathrm{E}-17$ & 131. & $8.52 \mathrm{E}-17$ & 152. & $7.12 \mathrm{E}-17$ & 204. \\
\hline $\mathrm{SI}+\mathrm{DS}$ & $1.35 \mathrm{E}-18$ & 91.1 & $1.50 \mathrm{E}-17$ & 95.1 & $4.16 \mathrm{E}-17$ & 170. & $4.22 \mathrm{E}-17$ & 207. & $4.30 \mathrm{E}-17$ & 237. & $3.80 \mathrm{E}-17$ & 320. \\
\hline SI+SPEX & $6.38 \mathrm{E}-18$ & 42.7 & $3.76 \mathrm{E}-17$ & 48.5 & $8.50 \mathrm{E}-17$ & 72.8 & $8.50 \mathrm{E}-17$ & 92.5 & $8.52 \mathrm{E}-17$ & 110. & $7.12 \mathrm{E}-17$ & 158. \\
\hline SI+DPEX & $2.69 \mathrm{E}-18$ & 78.4 & $1.73 \mathrm{E}-17$ & 90.9 & $3.26 \mathrm{E}-17$ & 122. & $3.57 \mathrm{E}-17$ & 144. & $2.91 \mathrm{E}-17$ & 163. & $3.06 \mathrm{E}-17$ & 208. \\
\hline SI SUM & $\ddagger 2.73 \mathrm{E}-17$ & & $1.08 \mathrm{E}-16$ & & $2.55 \mathrm{E}-16$ & & $2.57 \mathrm{E}-16$ & & $2.50 \mathrm{E}-16$ & & $2.17 \mathrm{E}-16$ & \\
\hline DI & $+1.00 \mathrm{E}-21$ & $\dagger 154$. & $7.14 \mathrm{E}-21$ & 96.5 & $8.56 \mathrm{E}-20$ & 151. & $4.61 \mathrm{E}-20$ & 201. & $1.64 \mathrm{E}-20$ & 246. & $1.53 \mathrm{E}-20$ & 362. \\
\hline $\mathrm{DI}+\mathrm{SS}$ & $4.61 \mathrm{E}-19$ & 175. & $5.33 \mathrm{E}-18$ & 121. & $2.42 \mathrm{E}-17$ & 196. & $2.62 \mathrm{E}-17$ & 252. & $2.58 \mathrm{E}-17$ & 300. & $1.91 \mathrm{E}-17$ & 303. \\
\hline $\mathrm{DI}+\mathrm{DS}$ & $6.52 \mathrm{E}-20$ & 215 . & $4.03 \mathrm{E}-18$ & 156. & $2.14 \mathrm{E}-17$ & 261. & $2.30 \mathrm{E}-17$ & 328. & $2.26 \mathrm{E}-17$ & 385. & $1.67 \mathrm{E}-17$ & 537. \\
\hline DI+SPEX & $4.61 \mathrm{E}-19$ & 166. & $5.35 \mathrm{E}-18$ & 109. & $2.43 \mathrm{E}-17$ & 164. & $2.62 \mathrm{E}-17$ & 214 & $2.58 \mathrm{E}-17$ & 259. & $1.91 \mathrm{E}-17$ & 375. \\
\hline DI+DPEX & $2.04 \mathrm{E}-19$ & 202. & $4.28 \mathrm{E}-18$ & 151. & $1.99 \mathrm{E}-17$ & 213. & $2.18 \mathrm{E}-17$ & 265. & $1.97 \mathrm{E}-17$ & 311. & $1.54 \mathrm{E}-17$ & 425. \\
\hline DI SUM & $1.19 \mathrm{E}-18$ & & $1.90 \mathrm{E}-17$ & & $8.99 \mathrm{E}-17$ & & $9.72 \mathrm{E}-17$ & & $9.39 \mathrm{E}-17$ & & $7.03 \mathrm{E}-17$ & \\
\hline TEX & $2.41 \mathrm{E}-20$ & 7.70 & $5.20 \mathrm{E}-19$ & 7.70 & $7.01 \mathrm{E}-18$ & 7.70 & $6.88 \mathrm{E}-18$ & 7.70 & $6.03 \mathrm{E}-18$ & 7.70 & $6.11 \mathrm{E}-18$ & 7.70 \\
\hline $\mathrm{TEX}+\mathrm{SS}$ & $1.04 \mathrm{E}-17$ & 34.7 & $4.16 \mathrm{E}-17$ & 31.7 & $4.03 \mathrm{E}-17$ & 52.6 & $3.76 \mathrm{E}-17$ & 58.2 & $3.76 \mathrm{E}-17$ & 61.5 & $3.39 \mathrm{E}-17$ & 66.5 \\
\hline TEX+DS & $4.13 \mathrm{E}-18$ & 68.4 & $1.85 \mathrm{E}-17$ & 66.8 & $1.76 \mathrm{E}-17$ & 118. & $1.62 \mathrm{E}-17$ & 134. & $1.62 \mathrm{E}-17$ & 147. & $1.46 \mathrm{E}-17$ & 182. \\
\hline TEX+SPEX & $1.04 \mathrm{E}-17$ & 20.0 & $4.19 \mathrm{E}-17$ & 20.2 & $4.06 \mathrm{E}-17$ & 20.2 & $3.79 \mathrm{E}-17$ & 20.2 & $3.76 \mathrm{E}-17$ & 20.2 & $3.39 \mathrm{E}-17$ & 20.2 \\
\hline TEX+DPEX & $6.91 \mathrm{E}-18$ & 55.7 & $2.14 \mathrm{E}-17$ & 62.6 & $1.35 \mathrm{E}-17$ & 69.4 & $1.34 \mathrm{E}-17$ & 71.9 & $1.06 \mathrm{E}-17$ & 72.6 & $1.15 \mathrm{E}-17$ & 70.9 \\
\hline TEX SUM & $3.19 \mathrm{E}-17$ & & $1.24 \mathrm{E}-16$ & & $1.19 \mathrm{E}-16$ & & $1.12 \mathrm{E}-16$ & & $1.08 \mathrm{E}-16$ & & $1.00 \mathrm{E}-16$ & \\
\hline \multirow{3}{*}{ Channel } & \multicolumn{12}{|l|}{ Energy } \\
\hline & $500 \mathrm{keV} / \mathrm{u}$ & & 1000 & & 2000 & & 5000 & & 10000 & & 25000 & \\
\hline & $\begin{array}{l}\text { cross } \\
\text { section } \\
\mathrm{cm}^{2}\end{array}$ & $\begin{array}{l}\text { Eloss } \\
\mathrm{eV}\end{array}$ & $\begin{array}{l}\text { cross } \\
\text { section } \\
\mathrm{cm}^{2}\end{array}$ & $\begin{array}{l}\text { Eloss } \\
\mathrm{eV}\end{array}$ & $\begin{array}{l}\text { cross } \\
\text { section } \\
\mathrm{cm}^{2}\end{array}$ & $\begin{array}{l}\text { Eloss } \\
\mathrm{eV}\end{array}$ & $\begin{array}{l}\text { cross } \\
\text { section } \\
\mathrm{cm}^{2}\end{array}$ & $\begin{array}{l}\text { Eloss } \\
\mathrm{eV}\end{array}$ & $\begin{array}{l}\text { cross } \\
\text { section } \\
\mathrm{cm}^{2}\end{array}$ & $\begin{array}{l}\text { Eloss } \\
\mathrm{eV}\end{array}$ & $\begin{array}{l}\text { cross } \\
\text { section } \\
\mathrm{cm}^{2}\end{array}$ & $\begin{array}{l}\text { Eloss } \\
\mathrm{eV}\end{array}$ \\
\hline SI & $2.93 \mathrm{E}-18$ & 189. & $1.64 \mathrm{E}-18$ & 203. & $5.32 \mathrm{E}-19$ & 205. & $3.27 \mathrm{E}-19$ & 192. & $2.02 \mathrm{E}-19$ & 182. & $6.55 \mathrm{E}-20$ & 168. \\
\hline $\mathrm{SI}+\mathrm{SS}$ & $5.28 \mathrm{E}-17$ & 250 & $4.09 \mathrm{E}-17$ & 264. & $3.14 \mathrm{E}-17$ & 264. & $2.07 \mathrm{E}-17$ & 250 & $1.43 \mathrm{E}-17$ & 241. & $8.51 \mathrm{E}-18$ & 226. \\
\hline $\mathrm{SI}+\mathrm{DS}$ & $3.06 \mathrm{E}-17$ & 408. & $2.51 \mathrm{E}-17$ & 444. & $1.78 \mathrm{E}-17$ & 449. & $1.32 \mathrm{E}-17$ & 426. & $9.15 \mathrm{E}-18$ & 416. & $4.36 \mathrm{E}-18$ & 411. \\
\hline SI+SPEX & $5.29 \mathrm{E}-17$ & 201. & $4.09 \mathrm{E}-17$ & 215. & $3.13 \mathrm{E}-17$ & 217. & $2.07 \mathrm{E}-17$ & 204. & $1.43 \mathrm{E}-17$ & 194. & $8.50 \mathrm{E}-18$ & 180. \\
\hline SI+DPEX & $2.77 \mathrm{E}-17$ & 255. & $2.15 \mathrm{E}-17$ & 267. & $1.65 \mathrm{E}-17$ & 264. & $8.22 \mathrm{E}-18$ & 257. & $5.76 \mathrm{E}-18$ & 248. & $3.76 \mathrm{E}-18$ & 230. \\
\hline SI SUM & $1.67 \mathrm{E}-16$ & & $1.30 \mathrm{E}-16$ & & $9.75 \mathrm{E}-17$ & & $6.31 \mathrm{E}-17$ & & $4.37 \mathrm{E}-17$ & & $2.52 \mathrm{E}-17$ & \\
\hline DI & $1.41 \mathrm{E}-21$ & 466. & $7.02 \mathrm{E}-22$ & 485. & $\dagger 3.00 \mathrm{E}-22$ & $\dagger 467$. & $\dagger 1.05 \mathrm{E}-22$ & $\dagger 420$ & $+4.70 \mathrm{E}-23$ & $\dagger 397$. & $\dagger 1.70 \mathrm{E}-23$ & +336 . \\
\hline $\mathrm{DI}+\mathrm{SS}$ & $1.03 \mathrm{E}-17$ & 527. & $6.01 \mathrm{E}-18$ & 546. & $3.35 \mathrm{E}-18$ & 526. & $1.41 \mathrm{E}-18$ & 478. & $6.72 \mathrm{E}-19$ & 456. & $2.38 \mathrm{E}-19$ & 394. \\
\hline $\mathrm{DI}+\mathrm{DS}$ & $9.05 \mathrm{E}-18$ & 685. & $5.33 \mathrm{E}-18$ & 726. & $2.81 \mathrm{E}-18$ & 711. & $1.24 \mathrm{E}-18$ & 654. & $5.84 \mathrm{E}-19$ & 631. & $1.62 \mathrm{E}-19$ & 579. \\
\hline DI+SPEX & $1.03 \mathrm{E}-17$ & 478. & $6.01 \mathrm{E}-18$ & 497. & $3.35 \mathrm{E}-18$ & 479. & $1.41 \mathrm{E}-18$ & 432. & $6.72 \mathrm{E}-19$ & 409. & $2.38 \mathrm{E}-19$ & 348. \\
\hline DI+DPEX & $8.68 \mathrm{E}-18$ & 532. & $4.96 \mathrm{E}-18$ & 549. & $2.70 \mathrm{E}-18$ & 526. & $9.22 \mathrm{E}-19$ & 485. & $4.31 \mathrm{E}-19$ & 463. & $1.62 \mathrm{E}-19$ & 398. \\
\hline DI SUM & $3.83 \mathrm{E}-17$ & & $2.23 \mathrm{E}-17$ & & $1.22 \mathrm{E}-17$ & & $4.98 \mathrm{E}-18$ & & $2.36 \mathrm{E}-18$ & & $8.00 \mathrm{E}-19$ & \\
\hline TEX & $2.54 \mathrm{E}-18$ & 7.70 & $9.90 \mathrm{E}-19$ & 7.70 & $1.22 \mathrm{E}-19$ & 7.70 & $2.34 \mathrm{E}-20$ & 7.70 & $9.66 \mathrm{E}-21$ & 7.70 & $+9.00 \mathrm{E}-22$ & 7.70 \\
\hline TEX+SS & $2.75 \mathrm{E}-17$ & 68.4 & $2.27 \mathrm{E}-17$ & 68.2 & $1.85 \mathrm{E}-17$ & 66.7 & $1.33 \mathrm{E}-17$ & 65.5 & $9.68 \mathrm{E}-18$ & 66.3 & $6.27 \mathrm{E}-18$ & 65.7 \\
\hline TEX+DS & $1.25 \mathrm{E}-17$ & 226 & $1.11 \mathrm{E}-17$ & 248. & $8.43 \mathrm{E}-18$ & 251. & $7.48 \mathrm{E}-18$ & 242. & $5.84 \mathrm{E}-18$ & 242. & $3.17 \mathrm{E}-18$ & 251. \\
\hline TEX+SPEX & $2.77 \mathrm{E}-17$ & 20.1 & $2.27 \mathrm{E}-17$ & 20.1 & $1.84 \mathrm{E}-17$ & 20.1 & $1.33 \mathrm{E}-17$ & 20.1 & $9.68 \mathrm{E}-18$ & 20.1 & $6.27 \mathrm{E}-18$ & 20.1 \\
\hline TEX+DPEX & $1.11 \mathrm{E}-17$ & 73.6 & $9.29 \mathrm{E}-18$ & 71.4 & $7.77 \mathrm{E}-18$ & 66.2 & $4.45 \mathrm{E}-18$ & 72.8 & $3.50 \mathrm{E}-18$ & 73.8 & $2.69 \mathrm{E}-18$ & 70.0 \\
\hline TEX SUM & $8.13 \mathrm{E}-17$ & & $6.68 \mathrm{E}-17$ & & $5.32 \mathrm{E}-17$ & & $3.86 \mathrm{E}-17$ & & $2.87 \mathrm{E}-17$ & & $1.84 \mathrm{E}-17$ & \\
\hline
\end{tabular}

$\dagger$ Extrapolated. $\ddagger$ Significantly improved. 
Table 4

The integral cross section and associated average energy loss for NSIM and SIM processes in $\mathrm{O}^{+}+\mathrm{H}_{2}$ collisions with impact energies between 1 and 25000 keV/u.

\begin{tabular}{|c|c|c|c|c|c|c|c|c|c|c|c|c|}
\hline \multirow[t]{2}{*}{ Channel } & \multicolumn{10}{|l|}{ Energy } & 200 & \\
\hline & $\begin{array}{l}\text { cross } \\
\text { section } \\
\mathrm{cm}^{2}\end{array}$ & $\begin{array}{l}\text { Eloss } \\
\mathrm{eV}\end{array}$ & $\begin{array}{l}\text { cross } \\
\text { section } \\
\mathrm{cm}^{2}\end{array}$ & $\begin{array}{l}\text { Eloss } \\
\mathrm{eV}\end{array}$ & $\begin{array}{l}\text { cross } \\
\text { section } \\
\mathrm{cm}^{2}\end{array}$ & $\begin{array}{l}\text { Eloss } \\
\mathrm{eV}\end{array}$ & $\begin{array}{l}\text { cross } \\
\text { section } \\
\mathrm{cm}^{2}\end{array}$ & $\begin{array}{l}\text { Eloss } \\
\mathrm{eV}\end{array}$ & $\begin{array}{l}\text { cross } \\
\text { section } \\
\mathrm{cm}^{2}\end{array}$ & $\begin{array}{l}\text { Eloss } \\
\mathrm{eV}\end{array}$ & $\begin{array}{l}\text { cross } \\
\text { section } \\
\mathrm{cm}^{2}\end{array}$ & $\begin{array}{l}\text { Eloss } \\
\mathrm{eV}\end{array}$ \\
\hline SI & $5.40 \mathrm{E}-21$ & 21.6 & $1.94 \mathrm{E}-18$ & 24.4 & $8.14 \mathrm{E}-17$ & 49.0 & $1.00 \mathrm{E}-16$ & 65.4 & $1.01 \mathrm{E}-16$ & 80.7 & $7.43 \mathrm{E}-17$ & 122. \\
\hline $\mathrm{SI}+\mathrm{SS}$ & $1.59 \mathrm{E}-19$ & 42.6 & $9.97 \mathrm{E}-18$ & 48.4 & $6.43 \mathrm{E}-17$ & 93.9 & $6.44 \mathrm{E}-17$ & 116. & $6.34 \mathrm{E}-17$ & 135. & $5.62 \mathrm{E}-17$ & 181. \\
\hline SI+DS & $1.08 \mathrm{E}-20$ & 82.3 & $4.52 \mathrm{E}-18$ & 83.5 & $1.88 \mathrm{E}-17$ & 159. & $2.34 \mathrm{E}-17$ & 192. & $2.63 \mathrm{E}-17$ & 220. & $2.72 \mathrm{E}-17$ & 297. \\
\hline SI+SPEX & $1.59 \mathrm{E}-19$ & 50.8 & $1.05 \mathrm{E}-17$ & 54.7 & $6.73 \mathrm{E}-17$ & 79.4 & $6.89 \mathrm{E}-17$ & 95.8 & $6.34 \mathrm{E}-17$ & 111. & $5.66 \mathrm{E}-17$ & 152. \\
\hline SI+DPEX & $1.51 \mathrm{E}-19$ & 62.6 & $9.20 \mathrm{E}-18$ & 71.1 & $3.82 \mathrm{E}-17$ & 96.4 & $4.50 \mathrm{E}-17$ & 113. & $4.37 \mathrm{E}-17$ & 128. & $4.17 \mathrm{E}-17$ & 169. \\
\hline SI SUM & $4.85 \mathrm{E}-19$ & & $3.61 \mathrm{E}-17$ & & $2.70 \mathrm{E}-16$ & & $3.02 \mathrm{E}-16$ & & $2.98 \mathrm{E}-16$ & & $2.56 \mathrm{E}-16$ & \\
\hline DI & $\dagger 5.00 \mathrm{E}-24$ & $\dagger 42.5$ & $\dagger 1.00 \mathrm{E}-20$ & $\dagger 55.5$ & $2.33 \mathrm{E}-18$ & 130. & $4.23 \mathrm{E}-18$ & 178. & $4.91 \mathrm{E}-18$ & 219. & $3.38 \mathrm{E}-18$ & 326. \\
\hline $\mathrm{DI}+\mathrm{SS}$ & $3.67 \mathrm{E}-22$ & 89.4 & $8.10 \mathrm{E}-19$ & 102. & $2.22 \mathrm{E}-17$ & 195. & $2.82 \mathrm{E}-17$ & 253. & $2.87 \mathrm{E}-17$ & 302. & $2.26 \mathrm{E}-17$ & 426. \\
\hline $\mathrm{DI}+\mathrm{DS}$ & $\dagger 1.00 \mathrm{E}-23$ & $\dagger 150$. & $5.93 \mathrm{E}-19$ & 157. & $1.45 \mathrm{E}-17$ & 282. & $2.01 \mathrm{E}-17$ & 350. & $2.16 \mathrm{E}-17$ & 406. & $1.77 \mathrm{E}-17$ & 556. \\
\hline DI+SPEX & $3.67 \mathrm{E}-22$ & 71.7 & $8.12 \mathrm{E}-19$ & 85.8 & $2.24 \mathrm{E}-17$ & 160. & $2.86 \mathrm{E}-17$ & 208. & $2.88 \mathrm{E}-17$ & 249. & $2.27 \mathrm{E}-17$ & 356. \\
\hline DI+DPEX & $3.67 \mathrm{E}-22$ & 83.5 & $8.05 E-19$ & 102. & $1.94 \mathrm{E}-17$ & 177. & $2.57 \mathrm{E}-17$ & 225. & $2.60 \mathrm{E}-17$ & 266. & $2.08 \mathrm{E}-17$ & 373. \\
\hline DI SUM & $1.12 \mathrm{E}-21$ & & $3.03 \mathrm{E}-18$ & & $8.08 \mathrm{E}-17$ & & $1.07 \mathrm{E}-16$ & & $1.10 \mathrm{E}-16$ & & $8.72 \mathrm{E}-17$ & \\
\hline $\mathrm{TI}$ & $7.53 \mathrm{E}-21$ & 14.8 & $4.15 \mathrm{E}-19$ & 22.8 & $3.77 \mathrm{E}-18$ & 82.0 & $2.07 \mathrm{E}-18$ & 122. & $1.07 \mathrm{E}-18$ & 160. & $5.55 \mathrm{E}-20$ & 277. \\
\hline $\mathrm{TI}+\mathrm{SS}$ & $2.11 \mathrm{E}-19$ & 61.7 & $9.16 \mathrm{E}-18$ & 68.9 & $2.39 \mathrm{E}-17$ & 147. & $1.55 \mathrm{E}-17$ & 197. & $9.91 \mathrm{E}-18$ & 243. & $2.35 \mathrm{E}-18$ & 377. \\
\hline $\mathrm{TI}+\mathrm{DS}$ & $1.65 \mathrm{E}-20$ & 122. & $4.73 \mathrm{E}-18$ & 124. & $1.46 \mathrm{E}-17$ & 234. & $1.14 \mathrm{E}-17$ & 294. & $7.97 \mathrm{E}-18$ & 347. & $2.10 \mathrm{E}-18$ & 507. \\
\hline TI+SPEX & $2.13 \mathrm{E}-19$ & 44.0 & $9.40 \mathrm{E}-18$ & 53.1 & $2.42 \mathrm{E}-17$ & 112. & $1.57 \mathrm{E}-17$ & 152. & $9.93 \mathrm{E}-18$ & 190. & $2.35 \mathrm{E}-18$ & 307. \\
\hline TI+DPEX & $2.05 E-19$ & 56.0 & $8.70 \mathrm{E}-18$ & 69.5 & $2.03 \mathrm{E}-17$ & 129. & $1.43 \mathrm{E}-17$ & 169. & $9.23 \mathrm{E}-18$ & 207. & $2.29 \mathrm{E}-18$ & 324. \\
\hline TI SUM & $6.53 \mathrm{E}-19$ & & $3.24 \mathrm{E}-17$ & & $8.68 \mathrm{E}-17$ & & $5.90 \mathrm{E}-17$ & & $3.81 \mathrm{E}-17$ & & $9.15 \mathrm{E}-18$ & \\
\hline SC & $4.86 \mathrm{E}-16$ & -7.47 & $2.81 \mathrm{E}-16$ & -2.57 & $4.81 \mathrm{E}-17$ & 19.2 & $1.53 \mathrm{E}-17$ & 32.8 & $6.09 \mathrm{E}-18$ & 46.4 & $1.68 \mathrm{E}-19$ & 101. \\
\hline $\mathrm{SC}+\mathrm{SS}$ & $3.78 \mathrm{E}-17$ & 39.4 & $8.28 \mathrm{E}-17$ & 43.5 & $3.23 \mathrm{E}-17$ & 84.4 & $1.48 \mathrm{E}-17$ & 108. & $8.38 \mathrm{E}-18$ & 129. & $1.83 \mathrm{E}-18$ & 201. \\
\hline $\mathrm{SC}+\mathrm{DS}$ & $6.87 \mathrm{E}-19$ & 99.8 & $1.50 \mathrm{E}-17$ & 98.5 & $7.78 \mathrm{E}-18$ & 171. & $5.56 \mathrm{E}-18$ & 205. & $3.96 \mathrm{E}-18$ & 233. & $1.28 \mathrm{E}-18$ & 331. \\
\hline SC+SPEX & $4.46 \mathrm{E}-17$ & 21.7 & $1.12 \mathrm{E}-16$ & 27.7 & $3.41 \mathrm{E}-17$ & 49.6 & $1.57 \mathrm{E}-17$ & 63.2 & $8.47 \mathrm{E}-18$ & 76.7 & $1.83 \mathrm{E}-18$ & 131. \\
\hline SC+DPEX & $2.58 \mathrm{E}-17$ & 33.5 & $6.10 \mathrm{E}-17$ & 44.1 & $1.77 \mathrm{E}-17$ & 66.6 & $1.05 \mathrm{E}-17$ & 80.1 & $6.20 \mathrm{E}-18$ & 93.2 & $1.64 \mathrm{E}-18$ & 148. \\
\hline SC SUM & $5.95 \mathrm{E}-16$ & & $5.52 \mathrm{E}-16$ & & $1.40 \mathrm{E}-16$ & & $6.19 \mathrm{E}-17$ & & $3.31 \mathrm{E}-17$ & & $6.75 \mathrm{E}-18$ & \\
\hline TEX & $4.02 \mathrm{E}-19$ & 7.70 & $1.10 \mathrm{E}-17$ & 7.70 & $5.83 \mathrm{E}-17$ & 7.70 & $6.86 \mathrm{E}-17$ & 7.70 & $7.84 \mathrm{E}-17$ & 7.70 & $7.28 \mathrm{E}-17$ & 7.70 \\
\hline $\mathrm{TEX}+\mathrm{SS}$ & $4.77 \mathrm{E}-18$ & 54.6 & $3.37 \mathrm{E}-17$ & 53.8 & $3.01 \mathrm{E}-17$ & 72.9 & $2.65 \mathrm{E}-17$ & 82.7 & $2.62 \mathrm{E}-17$ & 91.0 & $2.57 \mathrm{E}-17$ & 108. \\
\hline TEX+DS & $5.22 \mathrm{E}-19$ & 115. & $1.18 \mathrm{E}-17$ & 109. & $6.77 \mathrm{E}-18$ & 159. & $7.92 \mathrm{E}-18$ & 179. & $9.28 \mathrm{E}-18$ & 195. & $1.09 \mathrm{E}-17$ & 238. \\
\hline TEX+SPEX & $4.86 \mathrm{E}-18$ & 36.9 & $3.66 \mathrm{E}-17$ & 38.0 & $3.21 \mathrm{E}-17$ & 38.1 & $2.89 \mathrm{E}-17$ & 38.1 & $2.66 \mathrm{E}-17$ & 38.0 & $2.59 \mathrm{E}-17$ & 38.0 \\
\hline TEX+DPEX & $4.45 \mathrm{E}-18$ & 48.7 & $2.99 \mathrm{E}-17$ & 54.4 & $1.57 \mathrm{E}-17$ & 55.1 & $1.70 \mathrm{E}-17$ & 55.0 & $1.65 \mathrm{E}-17$ & 54.5 & $1.77 \mathrm{E}-17$ & 54.3 \\
\hline TEX SUM & $1.50 \mathrm{E}-17$ & & $1.23 \mathrm{E}-16$ & & $1.43 \mathrm{E}-16$ & & $1.49 \mathrm{E}-16$ & & $1.57 \mathrm{E}-16$ & & $1.53 \mathrm{E}-16$ & \\
\hline \multirow[t]{3}{*}{ Channel } & Energy & & & & & & & & & & & \\
\hline & $500 \mathrm{keV} / \mathrm{u}$ & & 1000 & & 2000 & & 5000 & & 10000 & & 25000 & \\
\hline & $\begin{array}{l}\text { cross } \\
\text { section } \\
\mathrm{cm}^{2}\end{array}$ & $\begin{array}{l}\text { Eloss } \\
\mathrm{eV}\end{array}$ & $\begin{array}{l}\text { cross } \\
\text { section } \\
\mathrm{cm}^{2}\end{array}$ & $\begin{array}{l}\text { Eloss } \\
\mathrm{eV}\end{array}$ & $\begin{array}{l}\text { cross } \\
\text { section } \\
\mathrm{cm}^{2}\end{array}$ & $\begin{array}{l}\text { Eloss } \\
\mathrm{eV}\end{array}$ & $\begin{array}{l}\text { cross } \\
\text { section } \\
\mathrm{cm}^{2}\end{array}$ & $\begin{array}{l}\text { Eloss } \\
\mathrm{eV}\end{array}$ & $\begin{array}{l}\text { cross } \\
\text { section } \\
\mathrm{cm}^{2}\end{array}$ & $\begin{array}{l}\text { Eloss } \\
\mathrm{eV}\end{array}$ & $\begin{array}{l}\text { cross } \\
\text { section } \\
\mathrm{cm}^{2}\end{array}$ & $\begin{array}{l}\text { Eloss } \\
\mathrm{eV}\end{array}$ \\
\hline SI & $4.24 \mathrm{E}-17$ & 165. & $2.60 \mathrm{E}-17$ & 184. & $1.51 \mathrm{E}-17$ & 190. & $8.99 \mathrm{E}-18$ & 184. & $4.32 \mathrm{E}-18$ & 176. & $1.79 \mathrm{E}-18$ & 163. \\
\hline SI+SS & $4.48 \mathrm{E}-17$ & 226 . & $3.66 \mathrm{E}-17$ & 245 . & $2.76 \mathrm{E}-17$ & 249. & $1.84 \mathrm{E}-17$ & 322. & $1.26 \mathrm{E}-17$ & 319. & $7.73 \mathrm{E}-18$ & 308. \\
\hline SI+DS & $2.25 \mathrm{E}-17$ & 384. & $1.86 \mathrm{E}-17$ & 425. & $1.56 \mathrm{E}-17$ & 434. & $9.00 \mathrm{E}-18$ & 518. & $6.64 \mathrm{E}-18$ & 525. & $3.01 \mathrm{E}-18$ & 547. \\
\hline SI+SPEX & $4.53 \mathrm{E}-17$ & 195. & $3.62 \mathrm{E}-17$ & 215 . & $2.80 \mathrm{E}-17$ & 220. & $1.83 \mathrm{E}-17$ & 214. & $1.25 \mathrm{E}-17$ & 206. & $7.67 \mathrm{E}-18$ & 193. \\
\hline SI+DPEX & $3.29 \mathrm{E}-17$ & 212. & $2.57 \mathrm{E}-17$ & 230. & $1.86 \mathrm{E}-17$ & 236. & $1.28 \mathrm{E}-17$ & 230. & $9.52 \mathrm{E}-18$ & 222. & $5.70 \mathrm{E}-18$ & 208. \\
\hline SI SUM & $1.88 \mathrm{E}-16$ & & $1.43 \mathrm{E}-16$ & & $1.05 \mathrm{E}-16$ & & $6.66 \mathrm{E}-17$ & & $4.56 \mathrm{E}-17$ & & $2.59 \mathrm{E}-17$ & \\
\hline DI & $1.34 \mathrm{E}-18$ & 427. & $5.61 \mathrm{E}-19$ & 452. & $2.28 \mathrm{E}-19$ & 441. & $6.78 \mathrm{E}-20$ & 405. & $1.67 \mathrm{E}-20$ & 367. & $1.11 \mathrm{E}-22$ & 331. \\
\hline $\mathrm{DI}+\mathrm{SS}$ & $1.24 \mathrm{E}-17$ & 543. & $7.08 \mathrm{E}-18$ & 578. & $3.75 \mathrm{E}-18$ & 572. & $1.52 \mathrm{E}-18$ & 543. & $6.98 \mathrm{E}-19$ & 510. & $2.43 E-19$ & 476. \\
\hline $\mathrm{DI}+\mathrm{DS}$ & $9.49 \mathrm{E}-18$ & 730. & $5.32 \mathrm{E}-18$ & 787. & $2.94 \mathrm{E}-18$ & 791. & $1.06 \mathrm{E}-18$ & 739. & $5.13 \mathrm{E}-19$ & 716. & $1.42 \mathrm{E}-19$ & 715. \\
\hline DI+SPEX & $1.24 \mathrm{E}-17$ & 457. & $7.06 \mathrm{E}-18$ & 482. & $3.76 \mathrm{E}-18$ & 471. & $1.52 \mathrm{E}-18$ & 435. & $6.97 \mathrm{E}-19$ & 397. & $2.43 \mathrm{E}-19$ & 361. \\
\hline DI+DPEX & $1.13 \mathrm{E}-17$ & 474. & $6.28 \mathrm{E}-18$ & 498. & $3.23 \mathrm{E}-18$ & 487. & $1.33 \mathrm{E}-18$ & 451. & $6.34 \mathrm{E}-19$ & 413. & $2.20 \mathrm{E}-19$ & 376. \\
\hline DI SUM & $4.69 \mathrm{E}-17$ & & $2.63 \mathrm{E}-17$ & & $1.39 \mathrm{E}-17$ & & $5.50 \mathrm{E}-18$ & & $2.56 \mathrm{E}-18$ & & $8.48 \mathrm{E}-19$ & \\
\hline $\mathrm{TI}$ & $1.76 \mathrm{E}-21$ & 511. & $6.34 \mathrm{E}-23$ & 794. & $\dagger 1.00 \mathrm{E}-24$ & $\dagger 1341$. & $\dagger 5.00 \mathrm{E}-27$ & $\dagger 2933$. & $+9.00 \mathrm{E}-29$ & $\dagger 5479$. & $\dagger 4.00 \mathrm{E}-31$ & $\dagger 13624$. \\
\hline $\mathrm{TI}+\mathrm{SS}$ & $1.65 \mathrm{E}-19$ & 627. & $1.49 \mathrm{E}-20$ & 920. & $1.07 \mathrm{E}-21$ & 1472. & $1.90 \mathrm{E}-23$ & 3071. & $1.10 \mathrm{E}-24$ & 5622. & $2.50 \mathrm{E}-26$ & 13769. \\
\hline $\mathrm{TI}+\mathrm{DS}$ & $1.59 \mathrm{E}-19$ & 814. & $1.47 \mathrm{E}-20$ & 1129. & $1.07 \mathrm{E}-21$ & 1692. & $1.90 \mathrm{E}-23$ & 3267. & $1.10 \mathrm{E}-24$ & 5828. & $2.50 \mathrm{E}-26$ & 14008. \\
\hline TI+SPEX & $1.65 \mathrm{E}-19$ & 541. & $1.49 \mathrm{E}-20$ & 824. & $1.07 \mathrm{E}-21$ & 1371. & $1.90 \mathrm{E}-23$ & 2963. & $1.10 \mathrm{E}-24$ & 5509. & $2.50 \mathrm{E}-26$ & 13654. \\
\hline TI+DPEX & $1.64 \mathrm{E}-19$ & 558. & $1.48 \mathrm{E}-20$ & 840. & $1.07 \mathrm{E}-21$ & 1387. & $1.90 \mathrm{E}-23$ & 2979. & $1.10 \mathrm{E}-24$ & 5525. & $2.50 \mathrm{E}-26$ & 13669. \\
\hline TI SUM & $6.55 \mathrm{E}-19$ & & $5.94 \mathrm{E}-20$ & & $4.28 \mathrm{E}-21$ & & $7.60 \mathrm{E}-23$ & & $4.40 \mathrm{E}-24$ & & $1.00 \mathrm{E}-25$ & \\
\hline SC & $\dagger 1.00 \mathrm{E}-21$ & $\dagger 264$. & $\dagger 1.00 \mathrm{E}-23$ & $\dagger 537$. & $\dagger 1.00 \mathrm{E}-25$ & $\dagger 1081$ & $\dagger 2.00 \mathrm{E}-28$ & $\dagger 2715$ & $\dagger 2.00 \mathrm{E}-30$ & $\dagger 5438$. & $\dagger 4.00 \mathrm{E}-33$ & $\dagger 13607$. \\
\hline $\mathrm{SC}+\mathrm{SS}$ & $1.52 \mathrm{E}-19$ & 380. & $1.86 \mathrm{E}-20$ & 663. & $1.96 \mathrm{E}-21$ & 1212. & $7.20 \mathrm{E}-23$ & 2853. & $2.95 \mathrm{E}-24$ & 5581. & $8.60 \mathrm{E}-26$ & 13752. \\
\hline $\mathrm{SC}+\mathrm{DS}$ & $1.38 \mathrm{E}-19$ & 567. & $1.83 \mathrm{E}-20$ & 872. & $1.96 \mathrm{E}-21$ & 1432. & $7.20 \mathrm{E}-23$ & 3049. & $2.95 \mathrm{E}-24$ & 5787. & $8.60 \mathrm{E}-26$ & 13991. \\
\hline SC+SPEX & $1.52 \mathrm{E}-19$ & 294. & $1.86 \mathrm{E}-20$ & 567. & $1.96 \mathrm{E}-21$ & 1111. & $7.20 \mathrm{E}-23$ & 2745 . & $2.95 \mathrm{E}-24$ & 5468. & $8.60 \mathrm{E}-26$ & 13637. \\
\hline SC+DPEX & $1.50 \mathrm{E}-19$ & 311. & $1.86 \mathrm{E}-20$ & 583. & $1.96 \mathrm{E}-21$ & 1127. & $7.20 \mathrm{E}-23$ & 2761. & $2.95 \mathrm{E}-24$ & 5484. & $8.60 \mathrm{E}-26$ & 13652. \\
\hline SC SUM & $5.93 \mathrm{E}-19$ & & $7.41 \mathrm{E}-20$ & & $7.84 \mathrm{E}-21$ & & $2.88 \mathrm{E}-22$ & & $1.18 \mathrm{E}-23$ & & $3.44 \mathrm{E}-25$ & \\
\hline TEX & $5.12 \mathrm{E}-17$ & 7.70 & $3.12 \mathrm{E}-17$ & 7.70 & $1.75 \mathrm{E}-17$ & 7.70 & $8.13 \mathrm{E}-18$ & 7.70 & $3.28 \mathrm{E}-18$ & 7.70 & $3.01 \mathrm{E}-19$ & 7.70 \\
\hline TEX+SS & $2.19 \mathrm{E}-17$ & 124. & $1.94 \mathrm{E}-17$ & 133. & $1.54 \mathrm{E}-17$ & 139. & $1.11 \mathrm{E}-17$ & 146. & $9.20 \mathrm{E}-18$ & 151. & $6.53 \mathrm{E}-18$ & 153. \\
\hline TEX+DS & $9.20 \mathrm{E}-18$ & 311. & $8.33 \mathrm{E}-18$ & 343. & $7.47 \mathrm{E}-18$ & 359. & $5.34 \mathrm{E}-18$ & 342. & $4.31 \mathrm{E}-18$ & 357. & $2.16 \mathrm{E}-18$ & 392. \\
\hline TEX+SPEX & $2.23 \mathrm{E}-17$ & 38.0 & $1.91 \mathrm{E}-17$ & 37.9 & $1.58 \mathrm{E}-17$ & 37.9 & $1.26 \mathrm{E}-17$ & 37.8 & $9.11 \mathrm{E}-18$ & 37.8 & $6.30 \mathrm{E}-18$ & 37.8 \\
\hline TEX+DPEX & $1.44 \mathrm{E}-17$ & 54.4 & $1.20 \mathrm{E}-17$ & 53.8 & $1.04 \mathrm{E}-17$ & 53.5 & $7.94 \mathrm{E}-18$ & 53.4 & $6.50 \mathrm{E}-18$ & 53.2 & $4.61 \mathrm{E}-18$ & 52.5 \\
\hline TEX SUM & $1.19 \mathrm{E}-16$ & & $9.00 \mathrm{E}-17$ & & $6.66 \mathrm{E}-17$ & & $4.51 \mathrm{E}-17$ & & $3.24 \mathrm{E}-17$ & & $1.99 \mathrm{E}-17$ & \\
\hline
\end{tabular}


Table 5

The integral cross section and associated average energy loss for NSIM and SIM processes in $\mathrm{O}^{2+}+\mathrm{H}_{2}$ collisions with impact energies between 1 and $25000 \mathrm{keV} / \mathrm{u}$.

\begin{tabular}{|c|c|c|c|c|c|c|c|c|c|c|c|c|}
\hline \multirow[t]{3}{*}{ Channel } & \multicolumn{12}{|l|}{ Energy } \\
\hline & \multicolumn{2}{|l|}{$1 \mathrm{keV} / \mathrm{u}$} & \multicolumn{2}{|l|}{10} & \multicolumn{2}{|l|}{50} & \multicolumn{2}{|l|}{75} & \multicolumn{2}{|l|}{100} & \multicolumn{2}{|l|}{200} \\
\hline & $\begin{array}{l}\text { cross } \\
\text { section } \\
\mathrm{cm}^{2}\end{array}$ & $\begin{array}{l}\text { Eloss } \\
\mathrm{eV}\end{array}$ & $\begin{array}{l}\text { cross } \\
\text { section } \\
\mathrm{cm}^{2}\end{array}$ & $\begin{array}{l}\text { Eloss } \\
\mathrm{eV}\end{array}$ & $\begin{array}{l}\text { cross } \\
\text { section } \\
\mathrm{cm}^{2}\end{array}$ & $\begin{array}{l}\text { Eloss } \\
\mathrm{eV}\end{array}$ & $\begin{array}{l}\text { cross } \\
\text { section } \\
\mathrm{cm}^{2}\end{array}$ & $\begin{array}{l}\text { Eloss } \\
\mathrm{eV}\end{array}$ & $\begin{array}{l}\text { cross } \\
\text { section } \\
\mathrm{cm}^{2}\end{array}$ & $\begin{array}{l}\text { Eloss } \\
\mathrm{eV}\end{array}$ & $\begin{array}{l}\text { cross } \\
\text { section } \\
\mathrm{cm}^{2}\end{array}$ & $\begin{array}{l}\text { Eloss } \\
\mathrm{eV}\end{array}$ \\
\hline SI & $1.05 \mathrm{E}-20$ & 22.7 & $2.00 \mathrm{E}-18$ & 23.4 & $* 2.57 \mathrm{E}-16$ & *32.6 & $* 3.50 \mathrm{E}-16$ & ${ }^{*} 41.0$ & $* 3.62 \mathrm{E}-16$ & $* 48.7$ & $* 2.73 \mathrm{E}-16$ & ${ }^{*} 69.2$ \\
\hline $\mathrm{SI}+\mathrm{SS}$ & $4.08 \mathrm{E}-20$ & 91.1 & $3.97 \mathrm{E}-18$ & 90.6 & $* 4.97 \mathrm{E}-17$ & * 115. & $* 6.24 \mathrm{E}-17$ & * 134. & $* 7.57 \mathrm{E}-17$ & * 151. & $* 7.00 \mathrm{E}-17$ & * 197. \\
\hline $\mathrm{SI}+\mathrm{DS}$ & $\dagger 2.40 \mathrm{E}-21$ & $\dagger 165$. & $4.98 \mathrm{E}-19$ & 168. & $* 1.60 \mathrm{E}-17$ & $* 225$ & $* 1.77 \mathrm{E}-17$ & $* 254$ & ${ }^{*} 2.61 \mathrm{E}-17$ & $* 277$ & $* 3.24 \mathrm{E}-17$ & ${ }^{*} 354$. \\
\hline SI+SPEX & $4.30 \mathrm{E}-20$ & 63.7 & $5.09 \mathrm{E}-18$ & 65.9 & ${ }^{*} 5.79 \mathrm{E}-17$ & ${ }^{*} 76.7$ & ${ }^{*} 6.98 \mathrm{E}-17$ & ${ }^{*} 84.9$ & ${ }^{*} 8.83 \mathrm{E}-17$ & ${ }^{*} 92.5$ & ${ }^{*} 8.22 \mathrm{E}-17$ & ${ }^{*} 113$. \\
\hline SI+DPEX & $5.37 \mathrm{E}-21$ & 120. & $1.94 \mathrm{E}-18$ & 131. & $* 1.75 \mathrm{E}-17$ & * 144. & $* 2.60 \mathrm{E}-17$ & $* 152$. & $* 2.55 \mathrm{E}-17$ & $* 160$. & $* 3.61 \mathrm{E}-17$ & * 179. \\
\hline SI SUM & $1.02 \mathrm{E}-19$ & & $1.35 \mathrm{E}-17$ & & $* 3.98 \mathrm{E}-16$ & & $* 5.26 \mathrm{E}-16$ & & $* 5.78 \mathrm{E}-16$ & & $* 4.94 \mathrm{E}-16$ & \\
\hline DI & $\dagger 1.00 \mathrm{E}-24$ & $\dagger 33.7$ & $2.44 \mathrm{E}-21$ & 54.8 & $8.91 \mathrm{E}-18$ & 116. & $1.96 \mathrm{E}-17$ & 157. & $2.14 \mathrm{E}-17$ & 190. & $1.71 \mathrm{E}-17$ & 276. \\
\hline $\mathrm{DI}+\mathrm{SS}$ & $\dagger 3.12 \mathrm{E}-24$ & $\dagger 102$ & $1.45 \mathrm{E}-19$ & 122. & $1.47 \mathrm{E}-17$ & 198. & $2.55 \mathrm{E}-17$ & 250. & $3.23 \mathrm{E}-17$ & 292. & $3.00 \mathrm{E}-17$ & 404. \\
\hline $\mathrm{DI}+\mathrm{DS}$ & $\dagger 6.92 \mathrm{E}-25$ & $\dagger 176$. & $2.98 \mathrm{E}-20$ & 199. & $8.89 \mathrm{E}-18$ & 308. & $1.47 \mathrm{E}-17$ & 370. & $2.07 \mathrm{E}-17$ & 418. & $2.15 \mathrm{E}-17$ & 560. \\
\hline DI+SPEX & $\dagger 3.12 \mathrm{E}-24$ & $\dagger 74.7$ & $1.51 \mathrm{E}-19$ & 97.3 & $1.54 \mathrm{E}-17$ & 160. & $2.64 \mathrm{E}-17$ & 201. & $3.41 \mathrm{E}-17$ & 234. & $3.17 \mathrm{E}-17$ & 320. \\
\hline DI+DPEX & $\dagger 2.08 \mathrm{E}-24$ & $\dagger 131$. & $1.08 \mathrm{E}-19$ & 163. & $9.32 \mathrm{E}-18$ & 227. & $1.78 \mathrm{E}-17$ & 268. & $2.05 \mathrm{E}-17$ & 301. & $2.27 \mathrm{E}-17$ & 386. \\
\hline DI SUM & $\dagger 1.00 \mathrm{E}-23$ & & $4.36 \mathrm{E}-19$ & & $5.72 \mathrm{E}-17$ & & $1.04 \mathrm{E}-16$ & & $1.29 \mathrm{E}-16$ & & $1.23 \mathrm{E}-16$ & \\
\hline TI & $1.36 \mathrm{E}-20$ & 0.85 & $9.50 \mathrm{E}-19$ & 15.4 & $3.10 \mathrm{E}-17$ & 80.5 & $2.24 \mathrm{E}-17$ & 118. & $1.01 \mathrm{E}-17$ & 132. & $7.19 \mathrm{E}-19$ & 242. \\
\hline $\mathrm{TI}+\mathrm{SS}$ & $8.73 \mathrm{E}-20$ & 69.3 & $6.03 \mathrm{E}-18$ & 82.6 & $3.66 \mathrm{E}-17$ & 163. & $3.04 \mathrm{E}-17$ & 211. & $2.19 \mathrm{E}-17$ & 234. & $4.67 \mathrm{E}-18$ & 370. \\
\hline $\mathrm{TI}+\mathrm{DS}$ & $\dagger 1.03 \mathrm{E}-20$ & $\dagger 143$. & $9.26 \mathrm{E}-19$ & 160. & $2.02 \mathrm{E}-17$ & 273. & $1.77 \mathrm{E}-17$ & 331. & $1.48 \mathrm{E}-17$ & 360. & $3.79 \mathrm{E}-18$ & 526. \\
\hline TI+SPEX & $9.62 \mathrm{E}-20$ & 41.9 & $7.18 \mathrm{E}-18$ & 57.9 & $3.90 \mathrm{E}-17$ & 125. & $3.12 \mathrm{E}-17$ & 162. & $2.28 \mathrm{E}-17$ & 176. & $4.79 \mathrm{E}-18$ & 286. \\
\hline TI+DPEX & $1.27 \mathrm{E}-20$ & 97.9 & $3.32 \mathrm{E}-18$ & 123. & $2.13 \mathrm{E}-17$ & 192. & $2.13 \mathrm{E}-17$ & 229. & $1.47 \mathrm{E}-17$ & 243. & $3.93 \mathrm{E}-18$ & 352. \\
\hline TI SUM & $2.20 \mathrm{E}-19$ & & $1.84 \mathrm{E}-17$ & & $1.48 \mathrm{E}-16$ & & $1.23 \mathrm{E}-16$ & & $8.43 \mathrm{E}-17$ & & $1.79 \mathrm{E}-17$ & \\
\hline DCAI & $1.39 \mathrm{E}-19$ & 19.4 & $1.58 \mathrm{E}-18$ & 41.9 & $7.10 \mathrm{E}-18$ & 93.1 & $1.80 \mathrm{E}-18$ & 118. & $3.71 \mathrm{E}-19$ & 140. & $2.05 \mathrm{E}-21$ & 222. \\
\hline $\mathrm{DCAI}+\mathrm{SS}$ & $6.85 \mathrm{E}-19$ & 87.8 & $4.42 \mathrm{E}-18$ & 109. & $4.44 \mathrm{E}-18$ & 175. & $1.84 \mathrm{E}-18$ & 211. & $6.84 \mathrm{E}-19$ & 242. & $2.07 \mathrm{E}-20$ & 350. \\
\hline DCAI+DS & $\dagger 8.19 \mathrm{E}-20$ & $\dagger 162$. & $8.02 \mathrm{E}-19$ & 187. & $2.14 \mathrm{E}-18$ & 285. & $9.48 \mathrm{E}-19$ & 331. & $4.46 \mathrm{E}-19$ & 368. & $1.71 \mathrm{E}-20$ & 506. \\
\hline DCAI+SPEX & $7.54 \mathrm{E}-19$ & 60.4 & $5.40 \mathrm{E}-18$ & 84.4 & $4.83 \mathrm{E}-18$ & 137. & $1.93 \mathrm{E}-18$ & 162. & $7.20 \mathrm{E}-19$ & 184. & $2.13 \mathrm{E}-20$ & 266. \\
\hline DCAI+DPEX & $9.09 \mathrm{E}-20$ & 116. & $2.60 \mathrm{E}-18$ & 150. & $2.29 \mathrm{E}-18$ & 204. & $1.18 \mathrm{E}-18$ & 229. & $4.41 \mathrm{E}-19$ & 251. & 1.77E-20 & 332. \\
\hline DCAI SUM & $1.75 \mathrm{E}-18$ & & $1.48 \mathrm{E}-17$ & & $2.08 \mathrm{E}-17$ & & $7.70 \mathrm{E}-18$ & & $2.66 \mathrm{E}-18$ & & $7.89 \mathrm{E}-20$ & \\
\hline SC & $7.48 \mathrm{E}-16$ & -21.1 & $6.18 \mathrm{E}-16$ & -16.2 & $2.40 \mathrm{E}-16$ & 26.2 & $9.28 \mathrm{E}-17$ & 39.8 & $3.50 \mathrm{E}-17$ & 53.4 & $1.64 \mathrm{E}-18$ & 87.3 \\
\hline $\mathrm{SC}+\mathrm{SS}$ & $1.72 \mathrm{E}-17$ & 47.3 & $6.63 \mathrm{E}-17$ & 51.0 & $3.75 \mathrm{E}-17$ & 109. & $2.34 \mathrm{E}-17$ & 133. & $1.49 \mathrm{E}-17$ & 156. & $2.93 \mathrm{E}-18$ & 215. \\
\hline $\mathrm{SC}+\mathrm{DS}$ & $\dagger 1.78 \mathrm{E}-18$ & $\dagger 121$. & $4.39 \mathrm{E}-18$ & 128. & $9.60 \mathrm{E}-18$ & 218. & $6.19 \mathrm{E}-18$ & 253. & $5.39 \mathrm{E}-18$ & 281. & $1.69 \mathrm{E}-18$ & 372. \\
\hline SC+SPEX & $2.54 \mathrm{E}-17$ & 19.9 & $1.40 \mathrm{E}-16$ & 26.3 & $4.50 \mathrm{E}-17$ & 70.3 & $2.62 \mathrm{E}-17$ & 83.7 & $1.71 \mathrm{E}-17$ & 97.2 & $3.20 \mathrm{E}-18$ & 131. \\
\hline SC+DPEX & $8.73 \mathrm{E}-19$ & 75.9 & $2.14 \mathrm{E}-17$ & 91.8 & $1.07 \mathrm{E}-17$ & 137. & $9.36 \mathrm{E}-18$ & 151. & $5.29 \mathrm{E}-18$ & 164. & $1.84 \mathrm{E}-18$ & 197. \\
\hline SC SUM & $7.93 \mathrm{E}-16$ & & $8.50 \mathrm{E}-16$ & & $3.43 \mathrm{E}-16$ & & $1.58 \mathrm{E}-16$ & & $7.77 \mathrm{E}-17$ & & $1.13 \mathrm{E}-17$ & \\
\hline DC & $4.86 \mathrm{E}-16$ & -20.4 & $4.56 \mathrm{E}-17$ & -10.6 & $8.23 \mathrm{E}-18$ & 62.6 & $1.80 \mathrm{E}-18$ & 89.8 & $4.86 \mathrm{E}-19$ & 117. & $2.70 \mathrm{E}-21$ & 196. \\
\hline $\mathrm{DC}+\mathrm{SS}$ & $4.08 \mathrm{E}-17$ & 48.0 & $2.03 \mathrm{E}-17$ & 56.6 & $9.64 \mathrm{E}-18$ & 145. & $4.28 \mathrm{E}-18$ & 183. & $2.46 \mathrm{E}-18$ & 219. & $1.23 \mathrm{E}-19$ & 324. \\
\hline $\mathrm{DC}+\mathrm{DS}$ & $\dagger 3.37 \mathrm{E}-18$ & $\dagger 122$. & $1.71 \mathrm{E}-18$ & 134. & $4.96 \mathrm{E}-18$ & 255. & $2.60 \mathrm{E}-18$ & 303. & $1.83 \mathrm{E}-18$ & 345. & $1.10 \mathrm{E}-19$ & 480. \\
\hline $\mathrm{DC}+\mathrm{SPEX}$ & $6.11 \mathrm{E}-17$ & 20.6 & $3.71 \mathrm{E}-17$ & 31.9 & $1.03 \mathrm{E}-17$ & 107. & $4.41 \mathrm{E}-18$ & 134. & $2.53 \mathrm{E}-18$ & 161. & $1.24 \mathrm{E}-19$ & 240. \\
\hline DC+DPEX & $2.33 \mathrm{E}-18$ & 76.6 & $7.30 \mathrm{E}-18$ & 97.4 & $5.24 \mathrm{E}-18$ & 174. & $3.11 \mathrm{E}-18$ & 201. & $1.81 \mathrm{E}-18$ & 228. & $1.13 \mathrm{E}-19$ & 306. \\
\hline DC SUM & $5.94 \mathrm{E}-16$ & & $1.12 \mathrm{E}-16$ & & $3.84 \mathrm{E}-17$ & & $1.62 \mathrm{E}-17$ & & $9.12 \mathrm{E}-18$ & & $4.73 \mathrm{E}-19$ & \\
\hline TEX & $4.88 \mathrm{E}-19$ & 7.70 & $1.47 \mathrm{E}-17$ & 7.70 & $1.26 \mathrm{E}-16$ & 7.70 & $1.55 \mathrm{E}-16$ & 7.70 & $1.87 \mathrm{E}-16$ & 7.70 & $2.03 \mathrm{E}-16$ & 7.70 \\
\hline TEX+SS & $1.97 \mathrm{E}-18$ & 76.1 & $2.63 \mathrm{E}-17$ & 74.9 & $1.53 \mathrm{E}-17$ & 90.0 & $1.74 \mathrm{E}-17$ & 101. & $2.08 \mathrm{E}-17$ & 110. & $2.27 \mathrm{E}-17$ & 136. \\
\hline TEX+DS & $\dagger 1.20 \mathrm{E}-19$ & $\dagger 150$ & $2.41 \mathrm{E}-18$ & 152. & $3.86 \mathrm{E}-18$ & 200. & $3.76 \mathrm{E}-18$ & 221. & $5.73 \mathrm{E}-18$ & 236. & $9.23 \mathrm{E}-18$ & 292. \\
\hline TEX+SPEX & $2.18 \mathrm{E}-18$ & 48.7 & $3.73 \mathrm{E}-17$ & 50.2 & $1.83 \mathrm{E}-17$ & 51.8 & $2.01 \mathrm{E}-17$ & 51.6 & $2.52 \mathrm{E}-17$ & 51.5 & $2.76 \mathrm{E}-17$ & 51.3 \\
\hline TEX+DPEX & $3.24 \mathrm{E}-19$ & 105. & $1.00 \mathrm{E}-17$ & 116. & $4.25 \mathrm{E}-18$ & 119. & $6.26 \mathrm{E}-18$ & 119. & $5.55 \mathrm{E}-18$ & 119. & $1.03 \mathrm{E}-17$ & 118. \\
\hline TEX SUM & $\S 5.08 \mathrm{E}-18$ & & $9.07 \mathrm{E}-17$ & & $1.68 \mathrm{E}-16$ & & $2.03 \mathrm{E}-16$ & & $2.44 \mathrm{E}-16$ & & $2.73 \mathrm{E}-16$ & \\
\hline \multirow[t]{4}{*}{ Channel } & Energy & & & & & & & & & & & \\
\hline & $500 \mathrm{keV} / \mathrm{u}$ & & 1000 & & 2000 & & 5000 & & 10000 & & 25000 & \\
\hline & cross & & cross & & cross & & cross & & cross & & cross & \\
\hline & $\begin{array}{l}\text { section } \\
\mathrm{cm}^{2}\end{array}$ & $\begin{array}{l}\text { Eloss } \\
\mathrm{eV}\end{array}$ & $\begin{array}{l}\text { section } \\
\mathrm{cm}^{2}\end{array}$ & $\begin{array}{l}\text { Eloss } \\
\mathrm{eV}\end{array}$ & $\begin{array}{l}\text { section } \\
\mathrm{cm}^{2}\end{array}$ & $\begin{array}{l}\text { Eloss } \\
\mathrm{eV}\end{array}$ & $\begin{array}{l}\text { section } \\
\mathrm{cm}^{2}\end{array}$ & $\begin{array}{l}\text { Eloss } \\
\mathrm{eV}\end{array}$ & $\begin{array}{l}\text { section } \\
\mathrm{cm}^{2}\end{array}$ & $\begin{array}{l}\text { Eloss } \\
\mathrm{eV}\end{array}$ & $\begin{array}{l}\text { section } \\
\mathrm{cm}^{2}\end{array}$ & $\begin{array}{l}\text { Eloss } \\
\mathrm{eV}\end{array}$ \\
\hline SI & $1.02 \mathrm{E}-16$ & 131. & $5.74 \mathrm{E}-17$ & 154. & $3.22 \mathrm{E}-17$ & 167. & $1.55 \mathrm{E}-17$ & 170. & $8.03 \mathrm{E}-18$ & 168. & $3.44 \mathrm{E}-18$ & 157. \\
\hline $\mathrm{SI}+\mathrm{SS}$ & $4.12 \mathrm{E}-17$ & 290. & $3.60 \mathrm{E}-17$ & 331. & $2.76 \mathrm{E}-17$ & 356. & $1.88 \mathrm{E}-17$ & 379. & $1.49 \mathrm{E}-17$ & 384. & $9.34 \mathrm{E}-18$ & 402. \\
\hline $\mathrm{SI}+\mathrm{DS}$ & $2.10 \mathrm{E}-17$ & 511. & $1.72 \mathrm{E}-17$ & 575. & $1.50 \mathrm{E}-17$ & 613. & $1.01 \mathrm{E}-17$ & 622. & $4.96 \mathrm{E}-18$ & 698. & $2.35 \mathrm{E}-18$ & 708. \\
\hline SI+SPEX & $4.54 \mathrm{E}-17$ & 174. & $3.65 \mathrm{E}-17$ & 197. & $2.79 \mathrm{E}-17$ & 210. & $1.88 \mathrm{E}-17$ & 213. & $1.49 \mathrm{E}-17$ & 211. & $9.42 \mathrm{E}-18$ & 200. \\
\hline SI+DPEX & $2.47 \mathrm{E}-17$ & 241. & $2.09 \mathrm{E}-17$ & 264. & $1.62 \mathrm{E}-17$ & 277. & $8.98 \mathrm{E}-18$ & 280. & $5.45 \mathrm{E}-18$ & 277. & $2.35 \mathrm{E}-18$ & 267. \\
\hline SI SUM & $2.34 \mathrm{E}-16$ & & $1.68 \mathrm{E}-16$ & & $1.19 \mathrm{E}-16$ & & $7.22 \mathrm{E}-17$ & & $4.82 \mathrm{E}-17$ & & $2.69 \mathrm{E}-17$ & \\
\hline DI & $6.98 \mathrm{E}-18$ & 370. & $2.73 \mathrm{E}-18$ & 401. & $1.02 \mathrm{E}-18$ & 402. & $2.70 \mathrm{E}-19$ & 374. & $7.33 \mathrm{E}-20$ & 355. & $1.15 \mathrm{E}-20$ & 322. \\
\hline $\mathrm{DI}+\mathrm{SS}$ & $1.59 \mathrm{E}-17$ & 529. & $8.78 \mathrm{E}-18$ & 578. & $4.59 \mathrm{E}-18$ & 591. & $1.82 \mathrm{E}-18$ & 583. & $9.25 \mathrm{E}-19$ & 571. & $3.25 \mathrm{E}-19$ & 567. \\
\hline $\mathrm{DI}+\mathrm{DS}$ & $1.15 \mathrm{E}-17$ & 750. & $6.81 \mathrm{E}-18$ & 822. & $3.36 \mathrm{E}-18$ & 848. & $1.30 \mathrm{E}-18$ & 826. & $4.54 \mathrm{E}-19$ & 885. & $1.26 \mathrm{E}-19$ & 873. \\
\hline DI+SPEX & $1.65 \mathrm{E}-17$ & 413. & $8.83 \mathrm{E}-18$ & 444. & $4.60 \mathrm{E}-18$ & 445. & $1.82 \mathrm{E}-18$ & 417. & $9.25 \mathrm{E}-19$ & 398. & $3.26 \mathrm{E}-19$ & 365. \\
\hline DI+DPEX & $1.26 \mathrm{E}-17$ & 480. & $6.76 \mathrm{E}-18$ & 511. & $3.53 \mathrm{E}-18$ & 512. & $1.18 \mathrm{E}-18$ & 484. & $4.93 \mathrm{E}-19$ & 464. & $1.26 \mathrm{E}-19$ & 432. \\
\hline DI SUM & $6.35 \mathrm{E}-17$ & & $3.39 \mathrm{E}-17$ & & $1.17 \mathrm{E}-17$ & & $6.39 \mathrm{E}-18$ & & $2.87 \mathrm{E}-18$ & & $9.15 \mathrm{E}-19$ & \\
\hline TI & $1.01 \mathrm{E}-20$ & 472. & $6.50 \mathrm{E}-22$ & 762. & $\dagger 2.00 \mathrm{E}-23$ & $\dagger 1309$. & $\dagger 1.50 \mathrm{E}-25$ & $\dagger 2803$. & $\dagger 4.00 \mathrm{E}-27$ & $\dagger 5511$. & $\dagger 3.00 \mathrm{E}-29$ & $\dagger 13664$. \\
\hline $\mathrm{TI}+\mathrm{SS}$ & $2.54 \mathrm{E}-19$ & 631. & $1.91 \mathrm{E}-20$ & 939. & $1.22 \mathrm{E}-21$ & 1498. & $2.42 \mathrm{E}-23$ & 3012. & $1.54 \mathrm{E}-24$ & 5727. & $3.00 \mathrm{E}-26$ & 13909. \\
\hline $\mathrm{TI}+\mathrm{DS}$ & $2.32 \mathrm{E}-19$ & 852. & $1.83 \mathrm{E}-20$ & 1183. & $1.20 \mathrm{E}-21$ & 1755. & $2.42 \mathrm{E}-23$ & 3355. & $1.54 \mathrm{E}-24$ & 6041. & $3.00 \mathrm{E}-26$ & 14215. \\
\hline TI+SPEX & $2.54 \mathrm{E}-19$ & 515. & $1.91 \mathrm{E}-20$ & 805. & $1.22 \mathrm{E}-21$ & 1352. & $2.42 \mathrm{E}-23$ & 2846. & $1.54 \mathrm{E}-24$ & 5554. & $3.00 \mathrm{E}-26$ & 13707. \\
\hline TI+DPEX & $2.40 \mathrm{E}-19$ & 582. & $1.88 \mathrm{E}-20$ & 872. & $1.21 \mathrm{E}-21$ & 1419. & $2.42 \mathrm{E}-23$ & 2913. & $1.54 \mathrm{E}-24$ & 5620. & $3.00 \mathrm{E}-26$ & 13774. \\
\hline TI SUM & $9.90 \mathrm{E}-19$ & & $7.60 \mathrm{E}-20$ & & $4.87 \mathrm{E}-21$ & & $9.70 \mathrm{E}-23$ & & $6.16 \mathrm{E}-24$ & & $1.20 \mathrm{E}-25$ & \\
\hline
\end{tabular}


Table 5 (continued)

\begin{tabular}{|c|c|c|c|c|c|c|c|c|c|c|c|c|}
\hline DCAI & $\dagger 1.00 \mathrm{E}-24$ & $\ddagger 438$. & $\dagger 1.00 \mathrm{E}-27$ & $\dagger 650$. & $\dagger 1.00 \mathrm{E}-30$ & $\dagger 1000$ & $\dagger 1.50 \mathrm{E}-34$ & $\dagger 1700$ & $\dagger 2.00 \mathrm{E}-37$ & $\dagger 2600$ & $\dagger 3.00 \mathrm{E}-41$ & $\dagger 4500$ \\
\hline $\mathrm{DCAI}+\mathrm{SS}$ & $4.43 \mathrm{E}-23$ & 597. & $2.50 \mathrm{E}-25$ & 827. & $1.25 \mathrm{E}-27$ & 1189. & $1.00 \mathrm{E}-30$ & 1991. & $5.00 \mathrm{E}-33$ & 2816. & $3.75 \mathrm{E}-36$ & 4745. \\
\hline DCAI+DS & $4.43 \mathrm{E}-23$ & 818. & $2.50 \mathrm{E}-25$ & 1071. & $1.25 \mathrm{E}-27$ & 1446. & $1.00 \mathrm{E}-30$ & 2152. & $5.00 \mathrm{E}-33$ & 3130. & $3.75 \mathrm{E}-36$ & 5051. \\
\hline DCAI+SPEX & $4.43 \mathrm{E}-23$ & 481. & $2.50 \mathrm{E}-25$ & 693. & $1.25 \mathrm{E}-27$ & 1043. & $1.00 \mathrm{E}-30$ & 1743. & $5.00 \mathrm{E}-33$ & 2643. & $3.75 \mathrm{E}-36$ & 4543. \\
\hline DCAI+DPEX & $4.43 \mathrm{E}-23$ & 548. & $2.50 \mathrm{E}-25$ & 760. & $1.25 \mathrm{E}-27$ & 1110. & $1.00 \mathrm{E}-30$ & 1810. & $5.00 \mathrm{E}-33$ & 2709. & $3.75 E-36$ & 4610. \\
\hline DCAI SUM & $\ddagger 1.78 \mathrm{E}-22$ & & $1.00 \mathrm{E}-24$ & & $5.00 \mathrm{E}-27$ & & $4.00 \mathrm{E}-30$ & & $2.00 \mathrm{E}-32$ & & $1.50 \mathrm{E}-35$ & \\
\hline SC & $1.56 \mathrm{E}-21$ & 251. & $+5.00 \mathrm{E}-24$ & $\dagger 523$. & $\dagger 5.00 \mathrm{E}-27$ & $\dagger 1068$. & $\dagger 2.00 \mathrm{E}-30$ & $\dagger 2701$ & $\dagger 7.00 \mathrm{E}-33$ & $\dagger 5425$ & $+2.00 \mathrm{E}-36$ & $\dagger 13594$. \\
\hline $\mathrm{SC}+\mathrm{SS}$ & $1.94 \mathrm{E}-19$ & 410. & $2.07 \mathrm{E}-20$ & 700. & $2.20 \mathrm{E}-21$ & 1257. & $6.28 \mathrm{E}-23$ & 2910. & $2.63 \mathrm{E}-24$ & 5641. & $1.00 \mathrm{E}-25$ & 13839. \\
\hline $\mathrm{SC}+\mathrm{DS}$ & $1.59 \mathrm{E}-19$ & 631. & $1.95 \mathrm{E}-20$ & 944. & $2.20 \mathrm{E}-21$ & 1514. & $6.28 \mathrm{E}-23$ & 3153. & $2.63 \mathrm{E}-24$ & 5955. & $1.00 \mathrm{E}-25$ & 14145. \\
\hline SC+SPEX & $1.95 \mathrm{E}-19$ & 294. & $2.07 \mathrm{E}-20$ & 566. & $2.20 \mathrm{E}-21$ & 1111. & $6.28 \mathrm{E}-23$ & 2744. & $2.63 \mathrm{E}-24$ & 5468. & $1.00 \mathrm{E}-25$ & 13637. \\
\hline SC+DPEX & $1.71 \mathrm{E}-19$ & 361. & $2.03 \mathrm{E}-20$ & 633. & $2.20 \mathrm{E}-21$ & 1178. & $6.28 \mathrm{E}-23$ & 2811. & $2.63 \mathrm{E}-24$ & 5534. & $1.00 \mathrm{E}-25$ & 13704. \\
\hline SC SUM & $7.21 \mathrm{E}-19$ & & $8.12 \mathrm{E}-20$ & & $8.80 \mathrm{E}-21$ & & $2.51 \mathrm{E}-22$ & & $1.05 \mathrm{E}-23$ & & $4.00 \mathrm{E}-25$ & \\
\hline DC & $\dagger 2.00 \mathrm{E}-24$ & $\dagger 523$. & $\dagger 1.00 \mathrm{E}-26$ & $\dagger 1068$. & $\dagger 1.00 \mathrm{E}-29$ & $\dagger 2157$. & $\dagger 2.00 \mathrm{E}-33$ & † 5425. & $\dagger 3.00 \mathrm{E}-36$ & $\dagger 10871$ & $\dagger 7.00 \mathrm{E}-40$ & $\dagger 27209$. \\
\hline $\mathrm{DC}+\mathrm{SS}$ & $1.04 \mathrm{E}-21$ & 682. & $1.33 \mathrm{E}-23$ & 1245. & $2.50 \mathrm{E}-27$ & 2346. & $5.00 \mathrm{E}-32$ & 5634. & $1.25 \mathrm{E}-35$ & 11087. & $2.50 \mathrm{E}-40$ & 27454 . \\
\hline $\mathrm{DC}+\mathrm{DS}$ & $1.01 \mathrm{E}-21$ & 903. & $1.31 \mathrm{E}-23$ & 1489. & $2.50 \mathrm{E}-27$ & 2603. & $5.00 \mathrm{E}-32$ & 5877. & $1.25 \mathrm{E}-35$ & 11401. & $2.50 \mathrm{E}-40$ & 27760 \\
\hline $\mathrm{DC}+\mathrm{SPEX}$ & $1.04 \mathrm{E}-21$ & 566. & $1.33 \mathrm{E}-23$ & 1111. & $2.50 \mathrm{E}-27$ & 2200. & $5.00 \mathrm{E}-32$ & 5468. & $1.25 \mathrm{E}-35$ & 10914. & $2.50 \mathrm{E}-40$ & 27252. \\
\hline DC+DPEX & $1.03 \mathrm{E}-21$ & 633. & $1.33 \mathrm{E}-23$ & 1178. & $2.50 \mathrm{E}-27$ & 2267. & $5.00 \mathrm{E}-32$ & 5535. & $1.25 \mathrm{E}-35$ & 10980. & $2.50 \mathrm{E}-40$ & 27319. \\
\hline DC SUM & $4.12 \mathrm{E}-21$ & & $5.30 \mathrm{E}-23$ & & $1.00 \mathrm{E}-26$ & & $2.02 \mathrm{E}-31$ & & $5.30 \mathrm{E}-35$ & & $1.70 \mathrm{E}-39$ & \\
\hline TEX & $1.53 \mathrm{E}-16$ & 7.70 & $9.17 \mathrm{E}-17$ & 7.70 & $4.93 \mathrm{E}-17$ & 7.70 & $1.93 \mathrm{E}-17$ & 7.70 & $9.00 \mathrm{E}-18$ & 7.70 & $2.49 \mathrm{E}-18$ & 7.70 \\
\hline $\mathrm{TEX}+\mathrm{SS}$ & $2.01 \mathrm{E}-17$ & 167. & $1.97 \mathrm{E}-17$ & 185. & $1.63 \mathrm{E}-17$ & 197. & $1.35 \mathrm{E}-17$ & 217. & $1.15 \mathrm{E}-17$ & 224 . & $8.27 \mathrm{E}-18$ & 253. \\
\hline $\mathrm{TEX}+\mathrm{DS}$ & $9.18 \mathrm{E}-18$ & 388. & $8.27 \mathrm{E}-18$ & 429. & $7.92 \mathrm{E}-18$ & 454. & $6.38 \mathrm{E}-18$ & 460. & $3.25 \mathrm{E}-18$ & 538. & $1.74 \mathrm{E}-18$ & 559. \\
\hline TEX+SPEX & $2.27 \mathrm{E}-17$ & 51.1 & $2.02 \mathrm{E}-17$ & 51.0 & $1.64 \mathrm{E}-17$ & 50.9 & $1.41 \mathrm{E}-17$ & 50.7 & $1.15 \mathrm{E}-17$ & 50.7 & $8.27 \mathrm{E}-18$ & 50.6 \\
\hline TEX+DPEX & $1.10 \mathrm{E}-17$ & 118. & $1.02 \mathrm{E}-17$ & 118. & $8.61 \mathrm{E}-18$ & 118. & $5.61 \mathrm{E}-18$ & 118. & $3.60 \mathrm{E}-18$ & 117. & $1.74 \mathrm{E}-18$ & 118. \\
\hline TEX SUM & $2.16 \mathrm{E}-16$ & & $1.50 \mathrm{E}-16$ & & $9.85 \mathrm{E}-17$ & & $5.89 \mathrm{E}-17$ & & $3.89 \mathrm{E}-17$ & & $2.25 \mathrm{E}-17$ & \\
\hline
\end{tabular}

\rceil Extrapolated. $\quad \ddagger$ Significantly improved. $\quad \S$ Corrects typographical error in Ref. [1]. $\quad{ }^{*}$ Includes Born correction. 
Table 6

The integral cross section and associated average energy loss for NSIM and SIM processes in $\mathrm{O}^{3+}+\mathrm{H}_{2}$ collisions with impact energies between 1 and $25000 \mathrm{keV} / \mathrm{u}$.

\begin{tabular}{|c|c|c|c|c|c|c|c|c|c|c|c|c|}
\hline \multirow[t]{3}{*}{ Channel } & \multicolumn{12}{|l|}{ Energy } \\
\hline & \multicolumn{2}{|l|}{$1 \mathrm{keV} / \mathrm{u}$} & \multicolumn{2}{|l|}{10} & \multicolumn{2}{|l|}{50} & \multicolumn{2}{|l|}{75} & \multicolumn{2}{|l|}{100} & \multicolumn{2}{|l|}{200} \\
\hline & $\begin{array}{l}\text { cross } \\
\text { section } \\
\mathrm{cm}^{2}\end{array}$ & $\begin{array}{l}\text { Eloss } \\
\mathrm{eV}\end{array}$ & $\begin{array}{l}\text { cross } \\
\text { section } \\
\mathrm{cm}^{2}\end{array}$ & $\begin{array}{l}\text { Eloss } \\
\mathrm{eV}\end{array}$ & $\begin{array}{l}\text { cross } \\
\text { section } \\
\mathrm{cm}^{2}\end{array}$ & $\begin{array}{l}\text { Eloss } \\
\mathrm{eV}\end{array}$ & $\begin{array}{l}\text { cross } \\
\text { section } \\
\mathrm{cm}^{2}\end{array}$ & $\begin{array}{l}\text { Eloss } \\
\mathrm{eV}\end{array}$ & $\begin{array}{l}\text { cross } \\
\text { section } \\
\mathrm{cm}^{2}\end{array}$ & $\begin{array}{l}\text { Eloss } \\
\mathrm{eV}\end{array}$ & $\begin{array}{l}\text { cross } \\
\text { section } \\
\mathrm{cm}^{2}\end{array}$ & $\begin{array}{l}\text { Eloss } \\
\mathrm{eV}\end{array}$ \\
\hline SI & $4.67 \mathrm{E}-20$ & $\ddagger 20.0$ & $1.76 \mathrm{E}-18$ & 22.8 & ${ }^{*} 4.26 \mathrm{E}-16$ & ${ }^{*} 24.5$ & $* 6.58 \mathrm{E}-16$ & $* 32.1$ & $* 7.88 \mathrm{E}-16$ & $* 36.7$ & $* 6.45 \mathrm{E}-16$ & $* 47.7$ \\
\hline $\mathrm{SI}+\mathrm{SS}$ & $2.08 \mathrm{E}-20$ & 112. & $1.56 \mathrm{E}-18$ & 114. & $* 3.80 \mathrm{E}-17$ & $* 129$. & $* 4.90 \mathrm{E}-17$ & * 146 . & $* 6.17 \mathrm{E}-17$ & $* 160$. & $* 7.05 \mathrm{E}-17$ & * 202 \\
\hline $\mathrm{SI}+\mathrm{DS}$ & $+8.34 \mathrm{E}-22$ & $\dagger 221$ & $\dagger 1.00 \mathrm{E}-19$ & $\dagger 244$ & ${ }^{*} 9.39 \mathrm{E}-18$ & $* 287$. & $* 9.51 \mathrm{E}-18$ & $* 308$. & $* 1.16 \mathrm{E}-17$ & $* 328$ & ${ }^{*} 1.91 \mathrm{E}-17$ & $* 400$ \\
\hline SI+SPEX & $2.08 \mathrm{E}-20$ & 67.8 & $2.40 \mathrm{E}-18$ & 75.1 & $* 4.43 \mathrm{E}-17$ & $* 81.2$ & $* 5.02 \mathrm{E}-17$ & $* 89.4$ & $* 7.05 \mathrm{E}-17$ & $* 93.7$ & $* 7.98 \mathrm{E}-17$ & $* 104$ \\
\hline SI+DPEX & $\dagger 4.17 \mathrm{E}-21$ & $\dagger 140$ & $3.98 \mathrm{E}-19$ & 170. & ${ }^{*} 1.47 \mathrm{E}-17$ & * 187. & ${ }^{*} 1.59 \mathrm{E}-17$ & * 196. & $* 2.13 \mathrm{E}-17$ & $* 205$ & $* 2.77 \mathrm{E}-17$ & ${ }^{*} 218$ \\
\hline SI SUM & $\ddagger 9.33 \mathrm{E}-20$ & & $6.22 \mathrm{E}-18$ & & ${ }^{*} 5.32 \mathrm{E}-16$ & & ${ }^{*} 7.83 \mathrm{E}-16$ & & $* 9.53 \mathrm{E}-16$ & & ${ }^{*} 8.42 \mathrm{E}-16$ & \\
\hline DI & $+1.00 \mathrm{E}-24$ & $\dagger 33.7$ & $+1.00 \mathrm{E}-21$ & $\dagger 56.4$ & $7.74 \mathrm{E}-18$ & 105. & $3.52 \mathrm{E}-17$ & 138. & $5.81 \mathrm{E}-17$ & 162. & $5.95 \mathrm{E}-17$ & 228. \\
\hline $\mathrm{DI}+\mathrm{SS}$ & $\dagger 3.68 \mathrm{E}-24$ & $\dagger 126$ & $4.10 \mathrm{E}-20$ & 148. & $6.63 \mathrm{E}-18$ & 210. & $1.81 \mathrm{E}-17$ & 252. & $2.91 \mathrm{E}-17$ & 285. & $3.91 \mathrm{E}-17$ & 382. \\
\hline $\mathrm{DI}+\mathrm{DS}$ & $+8.18 \mathrm{E}-25$ & $\dagger 235$ & $1.36 \mathrm{E}-20$ & 278. & $3.04 \mathrm{E}-18$ & 368. & $7.23 \mathrm{E}-18$ & 413. & $1.13 \mathrm{E}-17$ & 453. & $1.97 \mathrm{E}-17$ & 580. \\
\hline DI+SPEX & $+3.68 \mathrm{E}-24$ & $\dagger 81.5$ & $4.52 \mathrm{E}-20$ & 109. & $7.04 \mathrm{E}-18$ & 162. & $1.83 \mathrm{E}-17$ & 195. & $3.09 \mathrm{E}-17$ & 219. & $4.13 \mathrm{E}-17$ & 284. \\
\hline DI+DPEX & $\dagger 8.18 \mathrm{E}-25$ & $\dagger 154$ & $1.02 \mathrm{E}-20$ & 203. & $4.06 \mathrm{E}-18$ & 267. & $1.00 \mathrm{E}-17$ & 302. & $1.66 \mathrm{E}-17$ & 330. & $2.44 \mathrm{E}-17$ & 398. \\
\hline DI SUM & $\dagger 1.00 \mathrm{E}-23$ & & $1.11 \mathrm{E}-19$ & & $2.85 \mathrm{E}-17$ & & $8.88 \mathrm{E}-17$ & & $1.46 \mathrm{E}-16$ & & $1.84 \mathrm{E}-16$ & \\
\hline TI & $1.73 \mathrm{E}-20$ & 11.7 & $1.81 \mathrm{E}-18$ & 21.6 & $6.64 \mathrm{E}-17$ & 87.1 & $8.01 \mathrm{E}-17$ & 101. & $5.16 \mathrm{E}-17$ & 132. & $4.94 \mathrm{E}-18$ & 235. \\
\hline $\mathrm{TI}+\mathrm{SS}$ & $3.00 \mathrm{E}-20$ & 104. & $3.56 \mathrm{E}-18$ & 113. & $3.82 \mathrm{E}-17$ & 192. & $4.33 \mathrm{E}-17$ & 215. & $3.60 \mathrm{E}-17$ & 255. & $8.84 \mathrm{E}-18$ & 389. \\
\hline $\mathrm{TI}+\mathrm{DS}$ & $\dagger 5.62 \mathrm{E}-21$ & $\dagger 213$ & $\dagger 1.15 \mathrm{E}-18$ & $\dagger 243$. & $1.44 \mathrm{E}-17$ & 350. & $1.60 \mathrm{E}-17$ & 376. & $1.45 \mathrm{E}-17$ & 423. & $5.29 \mathrm{E}-18$ & 587. \\
\hline TI+SPEX & $3.00 \mathrm{E}-20$ & 59.5 & $4.93 \mathrm{E}-18$ & 73.9 & $4.16 \mathrm{E}-17$ & 144. & $4.38 \mathrm{E}-17$ & 158. & $3.80 \mathrm{E}-17$ & 189. & $9.15 \mathrm{E}-18$ & 291. \\
\hline TI+DPEX & $\dagger 3.74 \mathrm{E}-21$ & $\dagger 132$ & $1.04 \mathrm{E}-18$ & 169. & $2.04 \mathrm{E}-17$ & 249. & $2.28 \mathrm{E}-17$ & 265. & $2.10 \mathrm{E}-17$ & 300. & $6.27 \mathrm{E}-18$ & 405. \\
\hline TI SUM & $8.67 \mathrm{E}-20$ & & $1.25 \mathrm{E}-17$ & & $1.81 \mathrm{E}-16$ & & $2.06 \mathrm{E}-16$ & & $1.61 \mathrm{E}-16$ & & $3.45 \mathrm{E}-17$ & \\
\hline DCAI & $1.72 \mathrm{E}-16$ & 7.71 & $1.45 \mathrm{E}-16$ & 32.9 & $9.16 \mathrm{E}-17$ & 99.9 & $3.15 \mathrm{E}-17$ & 128. & $7.87 \mathrm{E}-18$ & 152. & $7.14 \mathrm{E}-20$ & 235. \\
\hline $\mathrm{DCAI}+\mathrm{SS}$ & $7.72 \mathrm{E}-18$ & 99.9 & $2.90 \mathrm{E}-17$ & 125. & $3.08 \mathrm{E}-17$ & 205. & $1.40 \mathrm{E}-17$ & 242. & $5.71 \mathrm{E}-18$ & 275. & $2.00 \mathrm{E}-19$ & 389. \\
\hline $\mathrm{DCAI}+\mathrm{DS}$ & $+4.53 \mathrm{E}-18$ & $\dagger 209$ & $+1.22 \mathrm{E}-17$ & $\dagger 254$ & $9.26 \mathrm{E}-18$ & 363. & $4.43 \mathrm{E}-18$ & 403. & $2.16 \mathrm{E}-18$ & 443. & $1.22 \mathrm{E}-19$ & 587. \\
\hline DCAI+SPEX & $1.19 \mathrm{E}-17$ & 55.5 & $4.87 \mathrm{E}-17$ & 85.0 & $3.46 \mathrm{E}-17$ & 157. & $1.42 \mathrm{E}-17$ & 185. & $6.09 \mathrm{E}-18$ & 209. & $2.05 \mathrm{E}-19$ & 291. \\
\hline DCAI+DPEX & $\dagger 1.81 \mathrm{E}-18$ & $\dagger 128$ & $\dagger 7.00 \mathrm{E}-18$ & $\dagger 180$ & $1.38 \mathrm{E}-17$ & 262. & $6.54 \mathrm{E}-18$ & 292. & $3.17 \mathrm{E}-18$ & 320. & $1.43 \mathrm{E}-19$ & 405. \\
\hline DCAI SUM & $1.98 \mathrm{E}-16$ & & $2.42 \mathrm{E}-16$ & & $1.80 \mathrm{E}-16$ & & $7.07 \mathrm{E}-17$ & & $2.50 \mathrm{E}-17$ & & $7.41 \mathrm{E}-19$ & \\
\hline SC & $1.36 \mathrm{E}-15$ & -7.92 & $1.04 \mathrm{E}-15$ & -3.02 & $5.51 \mathrm{E}-16$ & 18.8 & $2.74 \mathrm{E}-16$ & 32.4 & $1.16 \mathrm{E}-16$ & 46.0 & $6.91 \mathrm{E}-18$ & 101. \\
\hline $\mathrm{SC}+\mathrm{SS}$ & $8.71 \mathrm{E}-18$ & 84.3 & $3.51 \mathrm{E}-17$ & 88.6 & $3.69 \mathrm{E}-17$ & 124. & $2.59 \mathrm{E}-17$ & 147. & $1.74 \mathrm{E}-17$ & 169. & $3.85 \mathrm{E}-18$ & 255. \\
\hline $\mathrm{SC}+\mathrm{DS}$ & $+3.58 \mathrm{E}-18$ & $\dagger 193$. & $+7.74 \mathrm{E}-18$ & $\dagger 218$ & $4.87 \mathrm{E}-18$ & 281. & $3.02 \mathrm{E}-18$ & 308. & $2.43 \mathrm{E}-18$ & 337. & $1.26 \mathrm{E}-18$ & 453. \\
\hline SC+SPEX & $1.54 \mathrm{E}-17$ & 39.9 & $9.11 \mathrm{E}-17$ & 49.3 & $4.62 \mathrm{E}-17$ & 75.5 & $2.67 \mathrm{E}-17$ & 89.7 & $2.00 \mathrm{E}-17$ & 103. & $4.22 \mathrm{E}-18$ & 157. \\
\hline SC+DPEX & $\dagger 2.15 \mathrm{E}-18$ & $\dagger 112$ & $5.04 \mathrm{E}-18$ & 144. & $9.18 \mathrm{E}-18$ & 181. & $6.05 \mathrm{E}-18$ & 196. & $5.14 \mathrm{E}-18$ & 214. & $1.76 \mathrm{E}-18$ & 271. \\
\hline SC SUM & $1.39 \mathrm{E}-15$ & & $1.18 \mathrm{E}-15$ & & $6.48 \mathrm{E}-16$ & & $3.36 \mathrm{E}-16$ & & $1.61 \mathrm{E}-16$ & & $1.80 \mathrm{E}-17$ & \\
\hline DC & $6.41 \mathrm{E}-16$ & -3.60 & $1.67 \mathrm{E}-16$ & 6.21 & $2.45 \mathrm{E}-17$ & 49.8 & $8.04 \mathrm{E}-18$ & 77.0 & $2.09 \mathrm{E}-18$ & 104. & $2.89 \mathrm{E}-20$ & 213. \\
\hline $\mathrm{DC}+\mathrm{SS}$ & $1.66 \mathrm{E}-17$ & 88.6 & $3.03 \mathrm{E}-17$ & 97.8 & $2.15 \mathrm{E}-17$ & 155. & $1.08 \mathrm{E}-17$ & 191. & $5.05 \mathrm{E}-18$ & 227. & $3.29 \mathrm{E}-19$ & 367. \\
\hline $\mathrm{DC}+\mathrm{DS}$ & $+1.59 \mathrm{E}-17$ & $\dagger 198$ & $+1.63 \mathrm{E}-17$ & $\dagger 228$ & $7.20 \mathrm{E}-18$ & 312. & $4.33 \mathrm{E}-18$ & 352. & $2.46 \mathrm{E}-18$ & 395. & $2.39 \mathrm{E}-19$ & 565. \\
\hline $\mathrm{DC}+\mathrm{SPEX}$ & $3.02 \mathrm{E}-17$ & 44.2 & $6.77 \mathrm{E}-17$ & 58.5 & $2.36 \mathrm{E}-17$ & 107. & $1.09 \mathrm{E}-17$ & 134. & $5.23 \mathrm{E}-18$ & 161. & $3.33 \mathrm{E}-19$ & 269. \\
\hline $\mathrm{DC}+\mathrm{DPEX}$ & $+4.76 \mathrm{E}-18$ & $\dagger 116$ & $4.93 \mathrm{E}-18$ & 153. & $1.05 \mathrm{E}-17$ & 212. & $6.02 \mathrm{E}-18$ & 241. & $3.36 \mathrm{E}-18$ & 272. & $2.71 \mathrm{E}-19$ & 383. \\
\hline DC SUM & $7.08 \mathrm{E}-16$ & & $2.86 \mathrm{E}-16$ & & $8.73 \mathrm{E}-17$ & & $4.01 \mathrm{E}-17$ & & $1.82 \mathrm{E}-17$ & & $1.20 \mathrm{E}-18$ & \\
\hline TEX & $4.25 \mathrm{E}-19$ & 7.70 & $1.97 \mathrm{E}-17$ & 7.70 & $1.60 \mathrm{E}-16$ & 7.70 & $2.25 \mathrm{E}-16$ & 7.70 & $2.91 \mathrm{E}-16$ & 7.70 & $3.73 \mathrm{E}-16$ & 7.70 \\
\hline TEX+SS & $6.69 \mathrm{E}-19$ & 99.9 & $1.06 \mathrm{E}-17$ & 99.3 & $7.80 \mathrm{E}-18$ & 113. & $9.40 \mathrm{E}-18$ & 122. & $1.15 \mathrm{E}-17$ & 131. & $1.64 \mathrm{E}-17$ & 162. \\
\hline TEX+DS & $\dagger 1.05 \mathrm{E}-20$ & $\dagger 209$ & $\dagger 3.18 \mathrm{E}-19$ & $\dagger 229$ & $1.04 \mathrm{E}-18$ & 270. & $9.95 \mathrm{E}-19$ & 283. & $1.31 \mathrm{E}-18$ & 299. & $3.95 \mathrm{E}-18$ & 360. \\
\hline TEX+SPEX & $8.24 \mathrm{E}-19$ & 55.5 & $1.99 \mathrm{E}-17$ & 60.0 & $1.01 \mathrm{E}-17$ & 64.4 & $9.67 \mathrm{E}-18$ & 65.0 & $1.34 \mathrm{E}-17$ & 64.7 & $1.93 \mathrm{E}-17$ & 64.1 \\
\hline TEX+DPEX & $+4.20 \mathrm{E}-20$ & $\dagger 128$ & $2.10 \mathrm{E}-18$ & 155. & $1.95 \mathrm{E}-18$ & 170. & $1.96 \mathrm{E}-18$ & 172. & $2.78 \mathrm{E}-18$ & 176. & $5.55 \mathrm{E}-18$ & 178. \\
\hline TEX SUM & $1.97 \mathrm{E}-18$ & & $5.26 \mathrm{E}-17$ & & $\S 1.81 \mathrm{E}-16$ & & $2.47 \mathrm{E}-16$ & & $3.20 \mathrm{E}-16$ & & $4.18 \mathrm{E}-16$ & \\
\hline \multirow[t]{5}{*}{ Channel } & Energy & & & & & & & & & & & \\
\hline & $500 \mathrm{keV} / \mathrm{u}$ & & 1000 & & 2000 & & 5000 & & 10000 & & 25000 & \\
\hline & cross & & cross & & cross & & cross & & cross & & cross & \\
\hline & section & Eloss & section & Eloss & section & Eloss & section & Eloss & section & Eloss & section & Eloss \\
\hline & $\mathrm{cm}^{2}$ & $\mathrm{eV}$ & $\mathrm{cm}^{2}$ & $\mathrm{eV}$ & $\mathrm{cm}^{2}$ & $\mathrm{eV}$ & $\mathrm{cm}^{2}$ & $\mathrm{eV}$ & $\mathrm{cm}^{2}$ & $\mathrm{eV}$ & $\mathrm{cm}^{2}$ & $\mathrm{eV}$ \\
\hline SI & $* 3.33 \mathrm{E}-16$ & $* 67.5$ & $* 1.76 \mathrm{E}-16$ & $* 84.3$ & $* 7.84 \mathrm{E}-17$ & $* 99.5$ & $* 3.88 \mathrm{E}-17$ & $* 113$. & $1.36 \mathrm{E}-17$ & 154. & $5.75 \mathrm{E}-18$ & 150. \\
\hline $\mathrm{SI}+\mathrm{SS}$ & $* 6.22 \mathrm{E}-17$ & $* 267$. & $* 5.30 \mathrm{E}-17$ & $* 316$ & $* 3.76 \mathrm{E}-17$ & * 359. & $* 2.79 \mathrm{E}-17$ & * 399. & $1.33 \mathrm{E}-17$ & 475. & $8.64 \mathrm{E}-18$ & 496. \\
\hline $\mathrm{SI}+\mathrm{DS}$ & $* 2.38 \mathrm{E}-17$ & $* 530$. & $* 2.39 \mathrm{E}-17$ & ${ }^{*} 634$ & ${ }^{*} 1.87 \mathrm{E}-17$ & * 717. & ${ }^{*} 1.34 \mathrm{E}-17$ & *699. & $6.09 \mathrm{E}-18$ & 889. & $2.71 \mathrm{E}-18$ & 1014. \\
\hline SI+SPEX & $* 7.49 \mathrm{E}-17$ & * 123. & $* 6.25 \mathrm{E}-17$ & $* 140$. & $* 4.16 \mathrm{E}-17$ & * 155. & $* 2.82 \mathrm{E}-17$ & * 168. & $1.34 \mathrm{E}-17$ & 209. & $8.70 \mathrm{E}-18$ & 205. \\
\hline SI+DPEX & $* 3.07 \mathrm{E}-17$ & $* 237$ & $* 2.59 \mathrm{E}-17$ & $* 254$ & ${ }^{*} 1.66 \mathrm{E}-17$ & * 269. & $* 9.70 \mathrm{E}-18$ & $* 280$. & $6.21 \mathrm{E}-18$ & 323. & $2.71 \mathrm{E}-18$ & 321. \\
\hline SI SUM & $* 5.25 \mathrm{E}-16$ & & $* 3.41 \mathrm{E}-16$ & & ${ }^{*} 1.93 \mathrm{E}-16$ & & ${ }^{*} 1.18 \mathrm{E}-16$ & & $5.26 \mathrm{E}-17$ & & $2.85 \mathrm{E}-17$ & \\
\hline DI & $2.11 \mathrm{E}-17$ & 309. & $7.76 \mathrm{E}-18$ & 345. & $1.10 \mathrm{E}-18$ & 357. & $6.60 \mathrm{E}-19$ & 350. & $2.16 \mathrm{E}-19$ & 331. & $3.99 \mathrm{E}-20$ & 302. \\
\hline $\mathrm{DI}+\mathrm{SS}$ & $2.17 \mathrm{E}-17$ & 508. & $1.18 \mathrm{E}-17$ & 576. & $6.33 \mathrm{E}-18$ & 616. & $2.30 \mathrm{E}-18$ & 636. & $9.78 \mathrm{E}-19$ & 652. & $3.38 \mathrm{E}-19$ & 648. \\
\hline $\mathrm{DI}+\mathrm{DS}$ & $1.25 \mathrm{E}-17$ & 771. & $7.24 \mathrm{E}-18$ & 894. & $4.08 \mathrm{E}-18$ & 974. & $1.44 \mathrm{E}-18$ & 936. & $5.88 \mathrm{E}-19$ & 1066. & $1.51 \mathrm{E}-19$ & 1166. \\
\hline $\mathrm{DI}+\mathrm{SPEX}$ & $2.36 \mathrm{E}-17$ & 365. & $1.28 \mathrm{E}-17$ & 401. & $7.15 \mathrm{E}-18$ & 413. & $2.31 \mathrm{E}-18$ & 405. & $9.81 \mathrm{E}-19$ & 386. & $3.39 \mathrm{E}-19$ & 357. \\
\hline DI+DPEX & $1.47 \mathrm{E}-17$ & 478. & 7.67E-18 & 515. & $3.73 \mathrm{E}-18$ & 526. & $1.11 \mathrm{E}-18$ & 517. & $5.98 \mathrm{E}-19$ & 500. & $1.51 \mathrm{E}-19$ & 473. \\
\hline DI SUM & $9.36 \mathrm{E}-17$ & & $4.73 \mathrm{E}-17$ & & $2.24 \mathrm{E}-17$ & & $7.82 \mathrm{E}-18$ & & $3.36 \mathrm{E}-18$ & & $1.02 \mathrm{E}-18$ & \\
\hline TI & $6.16 \mathrm{E}-20$ & 428. & $3.55 \mathrm{E}-21$ & 717. & $2.49 \mathrm{E}-22$ & 1799. & $5.83 \mathrm{E}-24$ & 2915. & $3.50 \mathrm{E}-25$ & 5673. & $9.00 \mathrm{E}-27$ & 13842. \\
\hline $\mathrm{TI}+\mathrm{SS}$ & $4.10 \mathrm{E}-19$ & 627. & $2.76 \mathrm{E}-20$ & 948. & $1.66 \mathrm{E}-21$ & 2058. & $3.34 \mathrm{E}-23$ & 3201. & $1.40 \mathrm{E}-24$ & 5994. & $1.75 \mathrm{E}-26$ & 14188. \\
\hline $\mathrm{TI}+\mathrm{DS}$ & $3.15 \mathrm{E}-19$ & 890. & $2.42 \mathrm{E}-20$ & 1266. & $1.56 \mathrm{E}-21$ & 2416. & $3.09 \mathrm{E}-23$ & 3501. & $9.30 \mathrm{E}-25$ & 6408. & $1.75 \mathrm{E}-26$ & 14706. \\
\hline TI+SPEX & 4.16E-19 & 484. & $2.78 \mathrm{E}-20$ & 773. & $1.67 \mathrm{E}-21$ & 1855. & $3.34 \mathrm{E}-23$ & 2970. & $1.40 \mathrm{E}-24$ & 5728. & $1.75 \mathrm{E}-26$ & 13897. \\
\hline TI+DPEX & $3.48 \mathrm{E}-19$ & 597. & $2.49 \mathrm{E}-20$ & 887. & $1.51 \mathrm{E}-21$ & 1968. & $3.04 \mathrm{E}-23$ & 3082. & $9.30 \mathrm{E}-25$ & 5842. & $1.75 E-26$ & 14013. \\
\hline TI SUM & $1.55 \mathrm{E}-18$ & & $1.08 \mathrm{E}-19$ & & $6.65 \mathrm{E}-21$ & & $1.34 \mathrm{E}-22$ & & $5.01 \mathrm{E}-24$ & & $7.90 \mathrm{E}-26$ & \\
\hline
\end{tabular}


Table 6 (continued)

\begin{tabular}{|c|c|c|c|c|c|c|c|c|c|c|c|c|}
\hline DCAI & $\ddagger 5.00 \mathrm{E}-25$ & $\ddagger 450$. & $\ddagger 1.00 \mathrm{E}-28$ & $\ddagger 785$. & $\dagger 1.00 \mathrm{E}-32$ & $\dagger 1200$ & $\dagger 9.00 \mathrm{E}-38$ & $\dagger 1900$ & $\dagger 1.10 \mathrm{E}-41$ & $\dagger 3000$ & $\dagger 9.00 \mathrm{E}-47$ & $\dagger 5000$ \\
\hline $\mathrm{DCAI}+\mathrm{SS}$ & $4.28 \mathrm{E}-22$ & 649. & $1.53 \mathrm{E}-24$ & 1016. & $2.50 \mathrm{E}-27$ & 1459. & $3.75 E-31$ & 2186. & $6.24 \mathrm{E}-34$ & 3321. & $1.13 \mathrm{E}-37$ & 5346. \\
\hline $\mathrm{DCAI}+\mathrm{DS}$ & $3.57 \mathrm{E}-22$ & 912. & $1.53 \mathrm{E}-24$ & 1334. & $2.50 \mathrm{E}-27$ & 1817. & $3.75 \mathrm{E}-31$ & 2486. & $6.24 \mathrm{E}-34$ & 3735. & $1.13 \mathrm{E}-37$ & 5864. \\
\hline DCAI+SPEX & $4.36 \mathrm{E}-22$ & 506. & $1.53 \mathrm{E}-24$ & 841. & $2.50 \mathrm{E}-27$ & 1256. & $3.75 \mathrm{E}-31$ & 1955. & $6.24 \mathrm{E}-34$ & 3055. & $1.13 \mathrm{E}-37$ & 5055. \\
\hline DCAI+DPEX & $3.90 \mathrm{E}-22$ & 619. & $1.53 \mathrm{E}-24$ & 955. & $2.50 \mathrm{E}-27$ & 1369. & $3.75 \mathrm{E}-31$ & 2067. & $6.24 \mathrm{E}-34$ & 3169. & $1.13 \mathrm{E}-37$ & 5171. \\
\hline DCAI SUM & $\ddagger 1.61 \mathrm{E}-21$ & & $\ddagger 6.12 \mathrm{E}-24$ & & $1.00 \mathrm{E}-26$ & & $1.50 \mathrm{E}-30$ & & $2.50 \mathrm{E}-33$ & & $4.52 \mathrm{E}-37$ & \\
\hline SC & $1.61 \mathrm{E}-20$ & 234. & $5.15 \mathrm{E}-23$ & 506. & $\dagger 1.00 \mathrm{E}-26$ & $\dagger 1051$. & $\dagger 1.00 \mathrm{E}-30$ & $\dagger 2685$ & $+3.00 \mathrm{E}-34$ & $\dagger 5408$. & $\dagger 1.50 \mathrm{E}-38$ & $\dagger 13547$. \\
\hline $\mathrm{SC}+\mathrm{SS}$ & $2.45 \mathrm{E}-19$ & 433. & $2.36 \mathrm{E}-20$ & 737. & $2.22 \mathrm{E}-21$ & 1310. & $7.68 \mathrm{E}-23$ & 2971. & $3.35 \mathrm{E}-24$ & 5729. & $8.63 E-26$ & 13893. \\
\hline $\mathrm{SC}+\mathrm{DS}$ & $1.54 \mathrm{E}-19$ & 696. & $1.99 \mathrm{E}-20$ & 1055. & $2.16 \mathrm{E}-21$ & 1668. & $7.62 \mathrm{E}-23$ & 3271. & $3.35 \mathrm{E}-24$ & 6143. & $8.63 E-26$ & 14411. \\
\hline SC+SPEX & $2.54 \mathrm{E}-19$ & 290. & $2.36 \mathrm{E}-20$ & 562. & $2.22 \mathrm{E}-21$ & 1107. & $7.68 \mathrm{E}-23$ & 2740. & $3.35 \mathrm{E}-24$ & 5463. & $8.63 E-26$ & 13602. \\
\hline SC+DPEX & $1.80 \mathrm{E}-19$ & 403. & $2.05 \mathrm{E}-20$ & 676. & $2.08 \mathrm{E}-21$ & 1220. & $7.11 \mathrm{E}-23$ & 2852. & $3.35 \mathrm{E}-24$ & 5577. & $8.63 \mathrm{E}-26$ & 13718. \\
\hline SC SUM & $8.49 \mathrm{E}-19$ & & $8.77 \mathrm{E}-20$ & & $8.68 \mathrm{E}-21$ & & $3.01 \mathrm{E}-22$ & & $1.34 \mathrm{E}-23$ & & $3.45 \mathrm{E}-25$ & \\
\hline DC & $\dagger 5.00 \mathrm{E}-24$ & $\dagger 494$ & $\dagger 1.00 \mathrm{E}-26$ & $\dagger 1039$. & $\dagger 1.00 \mathrm{E}-29$ & $\dagger 2128$. & $\dagger 1.50 \mathrm{E}-33$ & † 5396. & $+2.00 \mathrm{E}-30$ & $\dagger 10842$ & $\dagger 2.00 \mathrm{E}-40$ & $\dagger 27181$. \\
\hline $\mathrm{DC}+\mathrm{SS}$ & $2.02 \mathrm{E}-21$ & 693. & $2.29 \mathrm{E}-23$ & 1270. & $2.50 \mathrm{E}-26$ & 2387. & $3.75 \mathrm{E}-30$ & 5682. & $5.00 \mathrm{E}-33$ & 11163. & $5.00 \mathrm{E}-37$ & 27527. \\
\hline $\mathrm{DC}+\mathrm{DS}$ & $1.77 \mathrm{E}-21$ & 956. & $2.29 \mathrm{E}-23$ & 1588. & $2.50 \mathrm{E}-26$ & 2745. & $3.75 \mathrm{E}-30$ & 5982. & $5.00 \mathrm{E}-33$ & 11577. & $5.00 \mathrm{E}-37$ & 28045 . \\
\hline $\mathrm{DC}+\mathrm{SPEX}$ & $2.02 \mathrm{E}-21$ & 550. & $2.29 \mathrm{E}-23$ & 1095. & $2.50 \mathrm{E}-26$ & 2184. & $3.75 \mathrm{E}-30$ & 5451. & $5.00 \mathrm{E}-33$ & 10897. & $5.00 \mathrm{E}-37$ & 27236 . \\
\hline DC+DPEX & $1.91 \mathrm{E}-21$ & 663. & $2.29 \mathrm{E}-23$ & 1209. & $2.50 \mathrm{E}-26$ & 2297. & $3.75 \mathrm{E}-30$ & 5563. & $5.00 \mathrm{E}-33$ & 11011. & $5.00 \mathrm{E}-37$ & 27352. \\
\hline DC SUM & $7.73 \mathrm{E}-21$ & & $9.16 \mathrm{E}-23$ & & $1.00 \mathrm{E}-25$ & & $1.50 \mathrm{E}-29$ & & $2.02 \mathrm{E}-30$ & & $2.00 \mathrm{E}-36$ & \\
\hline TEX & $3.08 \mathrm{E}-16$ & 7.70 & $2.03 \mathrm{E}-16$ & 7.70 & $1.12 \mathrm{E}-16$ & 7.70 & $4.54 \mathrm{E}-17$ & 7.70 & $2.07 \mathrm{E}-17$ & 7.70 & $6.18 \mathrm{E}-18$ & 7.70 \\
\hline $\mathrm{TEX}+\mathrm{SS}$ & $1.92 \mathrm{E}-17$ & 207. & $1.83 \mathrm{E}-17$ & 239. & $1.62 \mathrm{E}-17$ & 267. & $1.44 \mathrm{E}-17$ & 294. & $1.06 \mathrm{E}-17$ & 329. & $7.98 \mathrm{E}-18$ & 354. \\
\hline $\mathrm{TEX}+\mathrm{DS}$ & $6.98 \mathrm{E}-18$ & 470. & $7.75 \mathrm{E}-18$ & 557. & $8.09 \mathrm{E}-18$ & 625. & $6.28 \mathrm{E}-18$ & 594. & $4.27 \mathrm{E}-18$ & 743. & $2.14 \mathrm{E}-18$ & 872. \\
\hline TEX+SPEX & $2.34 \mathrm{E}-17$ & 63.6 & $2.20 \mathrm{E}-17$ & 63.3 & $1.99 \mathrm{E}-17$ & 63.2 & $1.46 \mathrm{E}-17$ & 62.8 & $1.07 \mathrm{E}-17$ & 62.7 & $8.05 \mathrm{E}-18$ & 62.6 \\
\hline TEX+DPEX & $9.16 \mathrm{E}-18$ & 177. & $8.43 \mathrm{E}-18$ & 178. & $7.17 \mathrm{E}-18$ & 177. & $4.50 \mathrm{E}-18$ & 175. & $4.36 \mathrm{E}-18$ & 177. & $2.14 \mathrm{E}-18$ & 179. \\
\hline TEX SUM & $3.67 \mathrm{E}-16$ & & $2.59 \mathrm{E}-16$ & & $1.63 \mathrm{E}-16$ & & $8.52 \mathrm{E}-17$ & & $5.06 \mathrm{E}-17$ & & $2.65 \mathrm{E}-17$ & \\
\hline
\end{tabular}

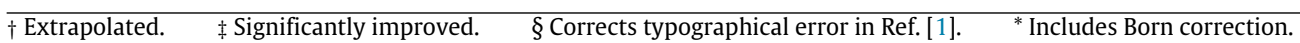


Table 7

The integral cross section and associated average energy loss for NSIM and SIM processes in $\mathrm{O}^{4+}+\mathrm{H}_{2}$ collisions with impact energies between 1 and $25000 \mathrm{keV} / \mathrm{u}$.

\begin{tabular}{|c|c|c|c|c|c|c|c|c|c|c|c|c|}
\hline \multirow[t]{2}{*}{ Channel } & \multicolumn{12}{|l|}{ Energy } \\
\hline & $\begin{array}{l}\text { cross } \\
\text { section } \\
\mathrm{cm}^{2}\end{array}$ & $\begin{array}{l}\text { Eloss } \\
\mathrm{eV}\end{array}$ & $\begin{array}{l}\text { cross } \\
\text { section } \\
\mathrm{cm}^{2}\end{array}$ & $\begin{array}{l}\text { Eloss } \\
\mathrm{eV}\end{array}$ & $\begin{array}{l}\text { cross } \\
\text { section } \\
\mathrm{cm}^{2}\end{array}$ & $\begin{array}{l}\text { Eloss } \\
\mathrm{eV}\end{array}$ & $\begin{array}{l}\text { cross } \\
\text { section } \\
\mathrm{cm}^{2}\end{array}$ & $\begin{array}{l}\text { Eloss } \\
\mathrm{eV}\end{array}$ & $\begin{array}{l}\text { cross } \\
\text { section } \\
\mathrm{cm}^{2}\end{array}$ & $\begin{array}{l}\text { Eloss } \\
\mathrm{eV}\end{array}$ & $\begin{array}{l}\text { cross } \\
\text { section } \\
\mathrm{cm}^{2}\end{array}$ & $\begin{array}{l}\text { Eloss } \\
\mathrm{eV}\end{array}$ \\
\hline SI & $2.73 \mathrm{E}-21$ & 27.5 & $2.33 \mathrm{E}-18$ & 13.4 & $* 6.87 \mathrm{E}-16$ & $* 20.9$ & $* 1.05 \mathrm{E}-15$ & $* 27.0$ & $* 1.34 \mathrm{E}-15$ & $* 31.9$ & $* 1.23 \mathrm{E}-15$ & $* 38.9$ \\
\hline $\mathrm{SI}+\mathrm{SS}$ & $9.63 \mathrm{E}-22$ & 161. & $6.70 \mathrm{E}-19$ & 144. & $* 1.36 \mathrm{E}-17$ & * 166. & $* 2.01 \mathrm{E}-17$ & * 180. & $* 2.91 \mathrm{E}-17$ & * 191. & $* 3.38 \mathrm{E}-17$ & ${ }^{*} 232$ \\
\hline $\mathrm{SI}+\mathrm{DS}$ & $\dagger 5.78 \mathrm{E}-23$ & $\dagger 295$ & $\dagger 5.12 \mathrm{E}-20$ & $\dagger 305$ & $* 5.25 \mathrm{E}-18$ & * 355. & $* 6.93 \mathrm{E}-18$ & $* 369$. & $* 1.02 \mathrm{E}-17$ & $* 384$ & $* 1.74 \mathrm{E}-17$ & $* 452$. \\
\hline SI+SPEX & $1.44 \mathrm{E}-21$ & 90.6 & $1.24 \mathrm{E}-18$ & 83.7 & $* 1.76 \mathrm{E}-17$ & * 99.1 & $* 2.19 \mathrm{E}-17$ & $* 108$. & $* 3.31 \mathrm{E}-17$ & $* 113$. & $* 2.10 \mathrm{E}-17$ & * 120. \\
\hline SI+DPEX & $2.60 \mathrm{E}-22$ & 168. & $2.29 \mathrm{E}-19$ & 181. & $* 9.83 \mathrm{E}-18$ & * 202. & $* 1.12 \mathrm{E}-17$ & $* 211$. & $* 1.39 \mathrm{E}-17$ & $* 218$. & $* 2.10 \mathrm{E}-17$ & $* 227$ \\
\hline SI SUM & $\dagger 5.45 \mathrm{E}-21$ & & $4.52 \mathrm{E}-18$ & & $* 7.33 \mathrm{E}-16$ & & $* 1.11 \mathrm{E}-15$ & & $* 1.43 \mathrm{E}-15$ & & $* 1.32 \mathrm{E}-15$ & \\
\hline DI & $\dagger 5.00 \mathrm{E}-25$ & $\dagger 35.7$ & $1.75 \mathrm{E}-21$ & 59.8 & $5.81 \mathrm{E}-18$ & 93.1 & $4.35 \mathrm{E}-17$ & 119. & $9.85 \mathrm{E}-17$ & 141. & $1.49 \mathrm{E}-16$ & 194. \\
\hline $\mathrm{DI}+\mathrm{SS}$ & $\dagger 1.29 \mathrm{E}-24$ & $\dagger 169$ & $1.74 \mathrm{E}-20$ & 191. & $1.52 \mathrm{E}-18$ & 238. & $7.19 \mathrm{E}-18$ & 272. & $1.57 \mathrm{E}-17$ & 300. & $3.07 \mathrm{E}-17$ & 387. \\
\hline $\mathrm{DI}+\mathrm{DS}$ & $\dagger 3.86 \mathrm{E}-25$ & $\dagger 303$ & $\dagger 5.46 \mathrm{E}-21$ & $\dagger 352$. & $7.85 \mathrm{E}-19$ & 427. & $3.56 \mathrm{E}-18$ & 461. & $7.97 \mathrm{E}-18$ & 493. & $2.08 \mathrm{E}-17$ & 607. \\
\hline $\mathrm{DI}+\mathrm{SPEX}$ & $\dagger 2.31 \mathrm{E}-24$ & $\dagger 98.8$ & $2.65 \mathrm{E}-20$ & 130. & $1.74 \mathrm{E}-18$ & 171. & $7.56 \mathrm{E}-18$ & 200. & $1.69 \mathrm{E}-17$ & 223. & $3.49 \mathrm{E}-17$ & 275 . \\
\hline DI+DPEX & $\dagger 5.14 \mathrm{E}-25$ & $\dagger 176$ & $6.62 \mathrm{E}-21$ & 228. & $1.24 \mathrm{E}-18$ & 274 . & $4.98 \mathrm{E}-18$ & 303. & $9.96 \mathrm{E}-18$ & 327. & $2.32 \mathrm{E}-17$ & 382. \\
\hline DI SUM & $\dagger 5.00 \mathrm{E}-24 \dagger$ & & $5.77 \mathrm{E}-20$ & & $1.11 \mathrm{E}-17$ & & $6.68 \mathrm{E}-17$ & & $1.49 \mathrm{E}-16$ & & $2.59 \mathrm{E}-16$ & \\
\hline $\mathrm{TI}$ & $2.77 \mathrm{E}-20$ & 4.48 & $3.65 \mathrm{E}-18$ & 13.1 & $1.04 \mathrm{E}-16$ & 52.2 & $1.77 \mathrm{E}-16$ & 83.4 & $1.48 \mathrm{E}-16$ & 113. & $1.74 \mathrm{E}-17$ & 211. \\
\hline $\mathrm{TI}+\mathrm{SS}$ & $1.22 \mathrm{E}-20$ & 138. & $1.93 \mathrm{E}-18$ & 144. & $1.81 \mathrm{E}-17$ & 197. & $3.03 \mathrm{E}-17$ & 236. & $3.26 \mathrm{E}-17$ & 272. & $1.28 \mathrm{E}-17$ & 404. \\
\hline $\mathrm{TI}+\mathrm{DS}$ & $\dagger 3.57 \mathrm{E}-21$ & $\dagger 271$ & $\dagger 6.10 \mathrm{E}-19$ & $\dagger 305$ & $8.21 \mathrm{E}-18$ & 386. & $1.41 \mathrm{E}-17$ & 425. & $1.65 \mathrm{E}-17$ & 465. & $9.36 \mathrm{E}-18$ & 624. \\
\hline TI+SPEX & $2.84 \mathrm{E}-20$ & 67.6 & $3.26 \mathrm{E}-18$ & 83.4 & $2.18 \mathrm{E}-17$ & 130. & $3.21 \mathrm{E}-17$ & 164. & $3.51 \mathrm{E}-17$ & 195. & $1.41 \mathrm{E}-17$ & 292. \\
\hline TI+DPEX & $\dagger 4.46 \mathrm{E}-21$ & $\dagger 144$ & $7.42 \mathrm{E}-19$ & 181. & $1.40 \mathrm{E}-17$ & 233. & $2.02 \mathrm{E}-17$ & 267. & $2.06 \mathrm{E}-17$ & 299. & $1.03 \mathrm{E}-17$ & 399. \\
\hline TI SUM & $7.63 \mathrm{E}-20$ & & $1.02 \mathrm{E}-17$ & & $1.66 \mathrm{E}-16$ & & $2.74 \mathrm{E}-16$ & & $2.53 \mathrm{E}-16$ & & $6.40 \mathrm{E}-17$ & \\
\hline DCAI & $6.44 \mathrm{E}-16$ & 10.0 & $4.89 \mathrm{E}-16$ & 35.9 & $3.21 \mathrm{E}-16$ & 105. & $1.39 \mathrm{E}-16$ & 138. & $4.49 \mathrm{E}-17$ & 163. & $7.38 \mathrm{E}-19$ & 246. \\
\hline $\mathrm{DCAI}+\mathrm{SS}$ & $6.56 \mathrm{E}-18$ & 143. & $2.92 \mathrm{E}-17$ & 167. & $3.42 \mathrm{E}-17$ & 250. & $2.03 \mathrm{E}-17$ & 291. & $1.04 \mathrm{E}-17$ & 322. & $5.26 \mathrm{E}-19$ & 439. \\
\hline $\mathrm{DCAI}+\mathrm{DS}$ & $\dagger 7.39 \mathrm{E}-18$ & $\dagger 277$ & $\dagger 1.65 \mathrm{E}-17$ & $\dagger 328$. & $1.32 \mathrm{E}-17$ & 439. & $8.41 \mathrm{E}-18$ & 480. & $5.02 \mathrm{E}-18$ & 515. & $4.00 \mathrm{E}-19$ & 659. \\
\hline DCAI+SPEX & $2.09 \mathrm{E}-17$ & 73.1 & $6.49 \mathrm{E}-17$ & 106. & $4.37 \mathrm{E}-17$ & 183. & $2.18 \mathrm{E}-17$ & 219. & $1.13 \mathrm{E}-17$ & 245 . & $5.74 \mathrm{E}-19$ & 327. \\
\hline DCAI+DPEX & $\dagger 3.69 \mathrm{E}-18$ & $\dagger 150$ & $9.13 \mathrm{E}-18$ & 204. & $2.47 \mathrm{E}-17$ & 286. & $1.26 \mathrm{E}-17$ & 322. & $6.34 \mathrm{E}-18$ & 349. & $4.33 \mathrm{E}-19$ & 434. \\
\hline DCAI SUM & $6.83 \mathrm{E}-16$ & & $6.09 \mathrm{E}-16$ & & $4.37 \mathrm{E}-16$ & & $2.02 \mathrm{E}-16$ & & $7.80 \mathrm{E}-17$ & & $2.67 \mathrm{E}-18$ & \\
\hline SC & $1.69 \mathrm{E}-15$ & -16.8 & $1.43 \mathrm{E}-15$ & -11.8 & $9.15 \mathrm{E}-16$ & 10.0 & $5.31 \mathrm{E}-16$ & 23.6 & $2.52 \mathrm{E}-16$ & 37.2 & $2.06 \mathrm{E}-17$ & 91.7 \\
\hline $\mathrm{SC}+\mathrm{SS}$ & $2.23 \mathrm{E}-18$ & 117. & $8.62 \mathrm{E}-18$ & 119. & $1.03 \mathrm{E}-17$ & 155. & $9.83 \mathrm{E}-18$ & 176. & $8.80 \mathrm{E}-18$ & 196. & $2.62 \mathrm{E}-18$ & 284. \\
\hline $\mathrm{SC}+\mathrm{DS}$ & $\dagger 1.20 \mathrm{E}-18$ & $\dagger 250$ & $\dagger 2.04 \mathrm{E}-18$ & $\dagger 280$ & $2.22 \mathrm{E}-18$ & 344. & $1.98 \mathrm{E}-18$ & 365. & $2.09 \mathrm{E}-18$ & 389. & $1.38 \mathrm{E}-18$ & 505. \\
\hline SC+SPEX & $1.08 \mathrm{E}-17$ & 46.3 & $2.88 \mathrm{E}-17$ & 58.5 & $1.59 \mathrm{E}-17$ & 88.2 & $1.12 \mathrm{E}-17$ & 104. & $1.04 \mathrm{E}-17$ & 119. & $3.26 \mathrm{E}-18$ & 173. \\
\hline SC+DPEX & $\dagger 1.20 \mathrm{E}-18$ & $\dagger 123$ & $2.11 \mathrm{E}-18$ & 156. & $5.96 \mathrm{E}-18$ & 191. & $4.04 \mathrm{E}-18$ & 208. & $3.30 \mathrm{E}-18$ & 223. & $1.65 \mathrm{E}-18$ & 280. \\
\hline SC SUM & $1.71 \mathrm{E}-15$ & & $1.47 \mathrm{E}-15$ & & $9.49 \mathrm{E}-16$ & & $5.58 \mathrm{E}-16$ & & $2.77 \mathrm{E}-16$ & & $2.95 \mathrm{E}-17$ & \\
\hline DC & $6.62 \mathrm{E}-16$ & -16.7 & $1.96 \mathrm{E}-16$ & -6.88 & $4.47 \mathrm{E}-17$ & 36.7 & $2.20 \mathrm{E}-17$ & 63.9 & $8.01 \mathrm{E}-18$ & 91.2 & $2.16 \mathrm{E}-19$ & 200. \\
\hline $\mathrm{DC}+\mathrm{SS}$ & $4.56 \mathrm{E}-18$ & 117. & $1.32 \mathrm{E}-17$ & 124. & $1.36 \mathrm{E}-17$ & 182. & $1.07 \mathrm{E}-17$ & 217. & $6.13 \mathrm{E}-18$ & 250 & $5.30 \mathrm{E}-19$ & 393. \\
\hline $\mathrm{DC}+\mathrm{DS}$ & $\dagger 1.25 \mathrm{E}-17$ & $\dagger 250$ & $\dagger 1.12 \mathrm{E}-17$ & $\dagger 285$ & $5.75 \mathrm{E}-18$ & 371. & $5.17 \mathrm{E}-18$ & 406. & $3.47 \mathrm{E}-18$ & 443. & $4.36 \mathrm{E}-19$ & 613. \\
\hline $\mathrm{DC}+\mathrm{SPEX}$ & $1.75 \mathrm{E}-17$ & 46.4 & $3.34 \mathrm{E}-17$ & 63.4 & $1.69 \mathrm{E}-17$ & 115. & $1.13 \mathrm{E}-17$ & 144. & $6.47 \mathrm{E}-18$ & 173. & $5.57 \mathrm{E}-19$ & 281. \\
\hline DC+DPEX & $\dagger 4.69 \mathrm{E}-18$ & $\dagger 123$ & $3.70 \mathrm{E}-18$ & 161. & $1.01 \mathrm{E}-17$ & 218. & $7.30 \mathrm{E}-18$ & 248. & $4.22 \mathrm{E}-18$ & 277. & $4.63 \mathrm{E}-19$ & 388. \\
\hline DC SUM & $7.01 \mathrm{E}-16$ & & $2.58 \mathrm{E}-16$ & & $9.11 \mathrm{E}-17$ & & $5.65 \mathrm{E}-01$ & & $2.83 \mathrm{E}-17$ & & $2.20 \mathrm{E}-18$ & \\
\hline TEX & $5.43 \mathrm{E}-19$ & 7.70 & $2.44 \mathrm{E}-17$ & 7.70 & $1.68 \mathrm{E}-16$ & 7.70 & $2.68 \mathrm{E}-16$ & 7.70 & $3.60 \mathrm{E}-16$ & 7.70 & $5.26 \mathrm{E}-16$ & 7.70 \\
\hline TEX+SS & $1.80 \mathrm{E}-19$ & 141. & $2.12 \mathrm{E}-18$ & 139. & $1.48 \mathrm{E}-18$ & 153. & $2.62 \mathrm{E}-18$ & 160. & $3.55 \mathrm{E}-18$ & 167. & $5.19 \mathrm{E}-18$ & 200. \\
\hline $\mathrm{TEX}+\mathrm{DS}$ & $\dagger 2.43 \mathrm{E}-21$ & $\dagger 275$ & $\dagger 6.31 \mathrm{E}-20$ & $\dagger 300$ & $2.84 \mathrm{E}-19$ & 342. & $4.53 \mathrm{E}-19$ & 349. & $8.40 \mathrm{E}-19$ & 360. & $2.45 \mathrm{E}-18$ & 421. \\
\hline TEX+SPEX & $4.40 \mathrm{E}-19$ & 70.8 & $5.22 \mathrm{E}-18$ & 78.0 & $2.18 \mathrm{E}-18$ & 85.9 & $1.11 \mathrm{E}-18$ & 88.2 & $4.12 \mathrm{E}-18$ & 89.2 & $7.23 \mathrm{E}-18$ & 88.5 \\
\hline TEX+DPEX & $\dagger 2.43 \mathrm{E}-20$ & $\dagger 148$ & $6.60 \mathrm{E}-19$ & 176. & $7.65 \mathrm{E}-19$ & 189. & $8.45 \mathrm{E}-19$ & 192. & $1.24 \mathrm{E}-18$ & 194. & $2.95 \mathrm{E}-18$ & 196. \\
\hline TEX SUM & $1.19 \mathrm{E}-18$ & & $3.25 \mathrm{E}-17$ & & $1.73 \mathrm{E}-16$ & & $2.73 \mathrm{E}-16$ & & $3.70 \mathrm{E}-16$ & & $5.44 \mathrm{E}-16$ & \\
\hline \multirow[t]{3}{*}{ Channel } & Energy & & & & & & & & & & & \\
\hline & $500 \mathrm{keV} / \mathrm{u}$ & & 1000 & & 2000 & & 5000 & & 10000 & & 25000 & \\
\hline & $\begin{array}{l}\text { cross } \\
\text { section } \\
\mathrm{cm}^{2}\end{array}$ & $\begin{array}{l}\text { Eloss } \\
\mathrm{eV}\end{array}$ & $\begin{array}{l}\text { cross } \\
\text { section } \\
\mathrm{cm}^{2}\end{array}$ & $\begin{array}{l}\text { Eloss } \\
\mathrm{eV}\end{array}$ & $\begin{array}{l}\text { cross } \\
\text { section } \\
\mathrm{cm}^{2}\end{array}$ & $\begin{array}{l}\text { Eloss } \\
\mathrm{eV}\end{array}$ & $\begin{array}{l}\text { cross } \\
\text { section } \\
\mathrm{cm}^{2}\end{array}$ & $\begin{array}{l}\text { Eloss } \\
\mathrm{eV}\end{array}$ & $\begin{array}{l}\text { cross } \\
\text { section } \\
\mathrm{cm}^{2}\end{array}$ & $\begin{array}{l}\text { Eloss } \\
\mathrm{eV}\end{array}$ & $\begin{array}{l}\text { cross } \\
\text { section } \\
\mathrm{cm}^{2}\end{array}$ & $\begin{array}{l}\text { Eloss } \\
\mathrm{eV}\end{array}$ \\
\hline SI & $* 6.85 \mathrm{E}-16$ & $* 48.9$ & $* 3.95 \mathrm{E}-16$ & $* 60.6$ & $* 2.05 \mathrm{E}-16$ & $* 73.3$ & $* 8.30 \mathrm{E}-17$ & $* 87.8$ & $* 4.21 \mathrm{E}-17$ & $* 94.5$ & $* 1.77 \mathrm{E}-17$ & $* 99.4$ \\
\hline $\mathrm{SI}+\mathrm{SS}$ & $* 3.93 \mathrm{E}-17$ & *306. & $* 3.54 \mathrm{E}-17$ & * 363. & $* 3.23 \mathrm{E}-17$ & $* 423$. & $* 2.61 \mathrm{E}-17$ & $* 487$ & ${ }^{*} 1.85 \mathrm{E}-17$ & * 539. & ${ }^{*} 1.06 \mathrm{E}-17$ & *630. \\
\hline $\mathrm{SI}+\mathrm{DS}$ & $* 2.14 \mathrm{E}-17$ & * 592 & $* 2.34 \mathrm{E}-17$ & $* 698$. & $* 2.23 \mathrm{E}-17$ & * 793. & $* 1.12 \mathrm{E}-17$ & $* 911$. & $* 7.39 \mathrm{E}-18$ & * 1007. & $* 4.40 \mathrm{E}-18$ & * 1379 \\
\hline SI+SPEX & $* 4.43 \mathrm{E}-17$ & * 129 & $* 3.96 \mathrm{E}-17$ & 140. & $* 3.53 \mathrm{E}-17$ & * 152. & $* 2.78 \mathrm{E}-17$ & $* 166$. & ${ }^{*} 1.94 \mathrm{E}-17$ & * 173 & $* 1.09 \mathrm{E}-17$ & $* 177$ \\
\hline SI+DPEX & $* 2.61 \mathrm{E}-17$ & *235. & $* 2.34 \mathrm{E}-17$ & $* 246$ & $* 2.23 \mathrm{E}-17$ & * 257. & $* 1.30 \mathrm{E}-17$ & $* 271$ & $* 8.61 \mathrm{E}-18$ & $* 278$. & $* 4.05 \mathrm{E}-18$ & $* 277$ \\
\hline SI SUM & $* 8.16 \mathrm{E}-16$ & & $* 5.17 \mathrm{E}-16$ & & $* 3.17 \mathrm{E}-01$ & & $* 1.61 \mathrm{E}-16$ & & $* 9.61 \mathrm{E}-17$ & & $* 4.77 \mathrm{E}-17$ & \\
\hline DI & $6.72 \mathrm{E}-17$ & 256. & $2.85 \mathrm{E}-17$ & 289. & $9.82 \mathrm{E}-18$ & 305. & $2.27 \mathrm{E}-18$ & 310. & $7.05 \mathrm{E}-19$ & 304. & $1.50 \mathrm{E}-19$ & 283. \\
\hline $\mathrm{DI}+\mathrm{SS}$ & $2.22 \mathrm{E}-17$ & 513. & $1.17 \mathrm{E}-17$ & 592. & $6.12 \mathrm{E}-18$ & 655. & $2.54 \mathrm{E}-18$ & 709. & $1.12 \mathrm{E}-18$ & 748. & $3.42 \mathrm{E}-19$ & 814. \\
\hline $\mathrm{DI}+\mathrm{DS}$ & $1.55 \mathrm{E}-17$ & 799. & $8.92 \mathrm{E}-18$ & 926. & $4.75 \mathrm{E}-18$ & 1025. & $1.33 \mathrm{E}-18$ & 1133. & $5.51 \mathrm{E}-19$ & 1216. & $1.77 \mathrm{E}-19$ & 1564. \\
\hline $\mathrm{DI}+\mathrm{SPEX}$ & $2.37 \mathrm{E}-17$ & 336. & $1.26 \mathrm{E}-17$ & 368. & $6.46 \mathrm{E}-18$ & 384. & $2.65 \mathrm{E}-18$ & 388. & $1.15 \mathrm{E}-18$ & 382. & $3.46 \mathrm{E}-19$ & 361. \\
\hline DI+DPEX & $1.75 \mathrm{E}-17$ & 442. & $8.92 \mathrm{E}-18$ & 474. & $4.75 \mathrm{E}-18$ & 489. & $1.51 \mathrm{E}-18$ & 493. & $6.30 \mathrm{E}-19$ & 487. & $1.65 \mathrm{E}-19$ & 461. \\
\hline DI SUM & $1.46 \mathrm{E}-16$ & & $7.06 \mathrm{E}-17$ & & $3.19 \mathrm{E}-17$ & & $1.03 \mathrm{E}-17$ & & $4.16 \mathrm{E}-18$ & & $1.18 \mathrm{E}-18$ & \\
\hline $\mathrm{TI}$ & $3.37 \mathrm{E}-19$ & 384. & $1.43 \mathrm{E}-20$ & 674. & $1.05 \mathrm{E}-21$ & 1235. & $6.00 \mathrm{E}-24$ & 2824. & $\dagger 1.40 \mathrm{E}-25$ & † 5513. & $\dagger 9.00 \mathrm{E}-28$ & $\dagger 13662$ \\
\hline $\mathrm{TI}+\mathrm{SS}$ & $6.09 \mathrm{E}-19$ & 641. & $3.75 \mathrm{E}-20$ & 977. & $2.09 \mathrm{E}-21$ & 1585. & $2.23 \mathrm{E}-23$ & 3223. & $3.43 \mathrm{E}-24$ & 5957. & $4.40 \mathrm{E}-27$ & 14193. \\
\hline $\mathrm{TI}+\mathrm{DS}$ & $5.01 \mathrm{E}-19$ & 927. & $3.41 \mathrm{E}-20$ & 1311. & $2.03 \mathrm{E}-21$ & 1955. & $2.23 \mathrm{E}-23$ & 3647. & $3.43 E-24$ & 6425. & $4.40 \mathrm{E}-27$ & 14942. \\
\hline $\mathrm{TI}+\mathrm{SPEX}$ & $6.25 \mathrm{E}-19$ & 464. & $3.80 \mathrm{E}-20$ & 753. & $2.10 \mathrm{E}-21$ & 1314. & $2.37 \mathrm{E}-23$ & 2902. & $3.43 \mathrm{E}-24$ & 5591. & $4.40 \mathrm{E}-27$ & 13740. \\
\hline TI+DPEX & $5.39 \mathrm{E}-19$ & 570. & $3.41 \mathrm{E}-20$ & 859. & $2.03 \mathrm{E}-21$ & 1419. & $2.12 \mathrm{E}-23$ & 3007. & $3.32 \mathrm{E}-24$ & 5696. & $4.40 \mathrm{E}-27$ & 13840. \\
\hline TI SUM & $2.61 \mathrm{E}-18$ & & $1.58 \mathrm{E}-19$ & & $9.30 \mathrm{E}-21$ & & $9.45 \mathrm{E}-23$ & & $1.38 \mathrm{E}-23$ & & $1.85 \mathrm{E}-26$ & \\
\hline
\end{tabular}


Table 7 (continued)

\begin{tabular}{|c|c|c|c|c|c|c|c|c|c|c|c|c|}
\hline DCAI & $1.76 \mathrm{E}-22$ & $\ddagger 459$. & $\dagger 1.00 \mathrm{E}-26$ & $\ddagger 779$. & $\dagger 1.00 \mathrm{E}-30$ & $\dagger 1200$ & $\dagger 7.00 \mathrm{E}-36$ & $\dagger 1950$ & $\dagger 6.00 \mathrm{E}-40$ & † 3100 & $\dagger 2.50 \mathrm{E}-45$ & $\dagger 5200$ \\
\hline $\mathrm{DCAI}+\mathrm{SS}$ & $1.21 \mathrm{E}-21$ & 716. & $3.85 \mathrm{E}-24$ & 1082. & $1.25 \mathrm{E}-26$ & 1550. & $6.25 E-30$ & 2349. & $1.75 \mathrm{E}-32$ & 3544. & $7.50 \mathrm{E}-36$ & 5731. \\
\hline $\mathrm{DCAI}+\mathrm{DS}$ & $1.07 \mathrm{E}-21$ & 1002. & $3.85 \mathrm{E}-24$ & 1416. & $1.25 \mathrm{E}-26$ & 1920. & $6.25 E-30$ & 2773. & $1.75 \mathrm{E}-32$ & 4012. & $7.50 \mathrm{E}-36$ & 6480. \\
\hline $\mathrm{DCAI}+\mathrm{SPEX}$ & $1.24 \mathrm{E}-21$ & 539. & $3.85 \mathrm{E}-24$ & 858. & $1.25 \mathrm{E}-26$ & 1279. & $6.25 E-30$ & 2028. & $1.75 \mathrm{E}-32$ & 3178. & $7.50 \mathrm{E}-36$ & 5278. \\
\hline DCAI+DPEX & $1.12 \mathrm{E}-21$ & 645. & $3.85 \mathrm{E}-24$ & 964. & $1.25 \mathrm{E}-26$ & 1384. & $6.25 \mathrm{E}-30$ & 2133. & $1.75 \mathrm{E}-32$ & 3283. & $7.50 \mathrm{E}-36$ & 5378. \\
\hline DCAI SUM & $\ddagger 4.82 \mathrm{E}-21$ & & $\ddagger 1.54 \mathrm{E}-23$ & & $5.00 \mathrm{E}-26$ & & $2.50 \mathrm{E}-29$ & & $7.00 \mathrm{E}-32$ & & $3.00 \mathrm{E}-35$ & \\
\hline SC & $1.92 \mathrm{E}-19$ & 214. & $2.07 \mathrm{E}-21$ & 487. & $\dagger 1.00 \mathrm{E}-24$ & $\dagger 1031$. & $\dagger 1.00 \mathrm{E}-28$ & $\dagger 2665$ & $\dagger 8.00 \mathrm{E}-32$ & $\dagger 5388$. & $\dagger 7.00 \mathrm{E}-36$ & $\dagger 13557$. \\
\hline $\mathrm{SC}+\mathrm{SS}$ & $2.56 \mathrm{E}-19$ & 471. & $2.68 \mathrm{E}-20$ & 790. & $2.33 \mathrm{E}-21$ & 1381. & $6.89 \mathrm{E}-23$ & 3064. & $4.88 \mathrm{E}-24$ & 5832. & $1.00 \mathrm{E}-25$ & 14088. \\
\hline $\mathrm{SC}+\mathrm{DS}$ & $1.79 \mathrm{E}-19$ & 757. & $2.34 \mathrm{E}-20$ & 1124. & $2.28 \mathrm{E}-21$ & 1751. & $6.00 \mathrm{E}-23$ & 3488. & $4.12 \mathrm{E}-24$ & 6300. & $1.00 \mathrm{E}-25$ & 14837. \\
\hline SC+SPEX & $2.70 \mathrm{E}-19$ & 294. & $2.73 \mathrm{E}-20$ & 566. & $2.33 \mathrm{E}-21$ & 1110. & $6.89 \mathrm{E}-23$ & 2743. & $4.88 \mathrm{E}-24$ & 5466. & $1.00 \mathrm{E}-25$ & 13635. \\
\hline SC+DPEX & $2.04 \mathrm{E}-19$ & 400. & $2.34 \mathrm{E}-20$ & 672. & $2.28 \mathrm{E}-21$ & 1215. & $6.62 \mathrm{E}-23$ & 2848. & $4.73 \mathrm{E}-24$ & 5571. & $1.00 \mathrm{E}-25$ & 13735. \\
\hline SC SUM & $1.10 \mathrm{E}-18$ & & $1.03 \mathrm{E}-19$ & & $9.22 \mathrm{E}-21$ & & $2.64 \mathrm{E}-22$ & & $1.86 \mathrm{E}-23$ & & $4.00 \mathrm{E}-25$ & \\
\hline DC & $2.18 \mathrm{E}-22$ & 465. & $\dagger 1.00 \mathrm{E}-25$ & $\dagger 1010$ & $\dagger 5.00 \mathrm{E}-29$ & $\dagger 2099$ & $\dagger 7.00 \mathrm{E}-33$ & $\dagger 5466$. & $\dagger 7.00 \mathrm{E}-36$ & $\dagger 10813$. & $\dagger 6.00 \mathrm{E}-40$ & $\dagger 27104$. \\
\hline $\mathrm{DC}+\mathrm{SS}$ & $4.74 \mathrm{E}-21$ & 722. & $5.31 \mathrm{E}-23$ & 1313. & $2.50 \mathrm{E}-25$ & 2449. & $2.50 \mathrm{E}-28$ & 5865. & $1.75 \mathrm{E}-30$ & 11257. & $2.25 \mathrm{E}-33$ & 27635. \\
\hline $\mathrm{DC}+\mathrm{DS}$ & $4.36 \mathrm{E}-21$ & 1008. & $5.24 \mathrm{E}-23$ & 1647. & $2.50 \mathrm{E}-25$ & 2819. & $2.50 \mathrm{E}-28$ & 6289. & $1.75 \mathrm{E}-30$ & 11725. & $2.25 \mathrm{E}-33$ & 28384. \\
\hline $\mathrm{DC}+\mathrm{SPEX}$ & $4.77 \mathrm{E}-21$ & 545. & $5.31 \mathrm{E}-23$ & 1089. & $2.50 \mathrm{E}-25$ & 2178. & $2.50 \mathrm{E}-28$ & 5544. & $1.75 \mathrm{E}-30$ & 10891. & $2.25 \mathrm{E}-33$ & 27182. \\
\hline DC+DPEX & $4.51 \mathrm{E}-21$ & 651. & $5.24 \mathrm{E}-23$ & 1195. & $2.50 \mathrm{E}-25$ & 2283. & $2.50 \mathrm{E}-28$ & 5649. & $1.75 \mathrm{E}-30$ & 10996. & $2.25 \mathrm{E}-33$ & 27282. \\
\hline DC SUM & $1.86 \mathrm{E}-20$ & & $2.11 \mathrm{E}-22$ & & $1.00 \mathrm{E}-24$ & & $1.00 \mathrm{E}-27$ & & $7.00 \mathrm{E}-30$ & & $9.00 \mathrm{E}-33$ & \\
\hline TEX & $5.33 \mathrm{E}-16$ & 7.70 & $3.69 \mathrm{E}-16$ & 7.70 & $2.22 \mathrm{E}-16$ & 7.70 & $9.65 \mathrm{E}-17$ & 7.70 & $4.48 \mathrm{E}-17$ & 7.70 & $1.51 \mathrm{E}-17$ & 7.70 \\
\hline $\mathrm{TEX}+\mathrm{SS}$ & $9.26 \mathrm{E}-18$ & 265. & $1.13 \mathrm{E}-17$ & 311. & $1.10 \mathrm{E}-17$ & 358. & $1.16 \mathrm{E}-17$ & 407. & $9.52 \mathrm{E}-18$ & 452. & $6.69 \mathrm{E}-18$ & 539. \\
\hline TEX+DS & $4.79 \mathrm{E}-18$ & 551. & $7.12 \mathrm{E}-18$ & 645. & $7.70 \mathrm{E}-18$ & 728. & $4.79 \mathrm{E}-18$ & 831. & $3.59 \mathrm{E}-18$ & 920. & $2.51 \mathrm{E}-18$ & 1288. \\
\hline TEX+SPEX & $1.05 \mathrm{E}-17$ & 87.6 & $1.26 \mathrm{E}-17$ & 86.9 & $1.22 \mathrm{E}-17$ & 86.6 & $1.25 \mathrm{E}-17$ & 86.0 & $1.01 \mathrm{E}-17$ & 85.8 & $6.88 \mathrm{E}-18$ & 85.6 \\
\hline TEX+DPEX & $6.13 \mathrm{E}-18$ & 194. & $7.12 \mathrm{E}-18$ & 193. & $7.70 \mathrm{E}-18$ & 192. & $5.59 \mathrm{E}-18$ & 191. & $4.19 \mathrm{E}-18$ & 191. & $2.30 \mathrm{E}-18$ & 186. \\
\hline TEX SUM & $5.64 \mathrm{E}-16$ & & $4.07 \mathrm{E}-16$ & & $2.61 \mathrm{E}-16$ & & $1.31 \mathrm{E}-16$ & & $7.22 \mathrm{E}-17$ & & $3.35 \mathrm{E}-17$ & \\
\hline
\end{tabular}

† Extrapolated. $\quad$ † Significantly improved. $\quad{ }^{*}$ Includes Born correction. 
Table 8

The integral cross section and associated average energy loss for NSIM and SIM processes in $0^{5+}+\mathrm{H}_{2}$ collisions with impact energies between 1 and $25000 \mathrm{keV} / \mathrm{u}$.

\begin{tabular}{|c|c|c|c|c|c|c|c|c|c|c|c|c|}
\hline \multirow[t]{2}{*}{ Channel } & \multicolumn{12}{|l|}{ Energy } \\
\hline & $\begin{array}{l}\text { cross } \\
\text { section } \\
\mathrm{cm}^{2}\end{array}$ & $\begin{array}{l}\text { Eloss } \\
\mathrm{eV}\end{array}$ & $\begin{array}{l}\text { cross } \\
\text { section } \\
\mathrm{cm}^{2}\end{array}$ & $\begin{array}{l}\text { Eloss } \\
\mathrm{eV}\end{array}$ & $\begin{array}{l}\text { cross } \\
\text { section } \\
\mathrm{cm}^{2}\end{array}$ & $\begin{array}{l}\text { Eloss } \\
\mathrm{eV}\end{array}$ & $\begin{array}{l}\text { cross } \\
\text { section } \\
\mathrm{cm}^{2}\end{array}$ & $\begin{array}{l}\text { Eloss } \\
\mathrm{eV}\end{array}$ & $\begin{array}{l}\text { cross } \\
\text { section } \\
\mathrm{cm}^{2}\end{array}$ & $\begin{array}{l}\text { Eloss } \\
\mathrm{eV}\end{array}$ & $\begin{array}{l}\text { cross } \\
\text { section } \\
\mathrm{cm}^{2}\end{array}$ & $\begin{array}{l}\text { Eloss } \\
\mathrm{eV}\end{array}$ \\
\hline SI & $2.35 \mathrm{E}-20$ & 28.9 & $1.67 \mathrm{E}-18$ & 23.0 & $* 3.44 \mathrm{E}-16$ & $* 22.0$ & $* 9.24 \mathrm{E}-16$ & $* 27.3$ & $* 1.37 \mathrm{E}-15$ & $* 32.7$ & $* 1.78 \mathrm{E}-15$ & $* 35.5$ \\
\hline $\mathrm{SI}+\mathrm{SS}$ & $\dagger 2.24 \mathrm{E}-21$ & $\dagger 178$ & $3.27 \mathrm{E}-19$ & 183. & ${ }^{*} 5.06 \mathrm{E}-18$ & * 194. & ${ }^{*} 1.45 \mathrm{E}-17$ & ${ }^{*} 206$. & $* 2.35 \mathrm{E}-17$ & $* 218$ & $* 4.45 \mathrm{E}-17$ & *253. \\
\hline $\mathrm{SI}+\mathrm{DS}$ & $\dagger 2.24 \mathrm{E}-25$ & +926 & $+5.82 \mathrm{E}-23$ & † 965. & $*+8.33 \mathrm{E}-21$ & $*+1049$ & ${ }^{*}+3.20 \mathrm{E}-20$ & $*+1105$ & $* 5.98 \mathrm{E}-20$ & ${ }^{*} 1144$ & ${ }^{*} 1.95 \mathrm{E}-19$ & * 1250 \\
\hline SI+SPEX & $\dagger 5.59 \mathrm{E}-21$ & $\dagger 92.2$ & $1.54 \mathrm{E}-18$ & 94.1 & $* 7.23 \mathrm{E}-18$ & $* 105$. & ${ }^{*} 1.84 \mathrm{E}-17$ & $* 114$. & $* 2.93 \mathrm{E}-17$ & ${ }^{*} 121$. & $* 5.36 \mathrm{E}-17$ & $* 124$ \\
\hline SI+DPEX & $\dagger 1.12 \mathrm{E}-24$ & $\dagger 579$. & $\dagger 2.91 \mathrm{E}-22$ & $\dagger 643$. & $* 4.64 \mathrm{E}-20$ & ${ }^{*} 683$ & ${ }^{*} 1.45 \mathrm{E}-19$ & ${ }^{*} 689$ & $* 2.13 \mathrm{E}-19$ & ${ }^{*} 688$ & $* 9.34 \mathrm{E}-19$ & ${ }^{*} 721$. \\
\hline SI SUM & $3.13 \mathrm{E}-20$ & & $3.54 \mathrm{E}-18$ & & $* 3.56 \mathrm{E}-16$ & & ${ }^{*} 9.57 \mathrm{E}-16$ & & $* 1.42 \mathrm{E}-15$ & & ${ }^{*} 1.88 \mathrm{E}-15$ & \\
\hline DI & $\dagger 1.00 \mathrm{E}-25$ & $\dagger 35.7$ & $1 \dagger .00 \mathrm{E}-23$ & $\dagger 51.7$ & $3.17 \mathrm{E}-18$ & 79.3 & $3.56 \mathrm{E}-17$ & 103. & $1.09 \mathrm{E}-16$ & 126. & $2.34 \mathrm{E}-16$ & 174. \\
\hline $\mathrm{DI}+\mathrm{SS}$ & $+1.96 \mathrm{E}-25$ & $\dagger 185$ & $9.97 \mathrm{E}-21$ & 212. & $6.28 \mathrm{E}-19$ & 251. & $5.17 \mathrm{E}-18$ & 282. & $1.51 \mathrm{E}-17$ & 311. & $4.76 \mathrm{E}-17$ & 392. \\
\hline $\mathrm{DI}+\mathrm{DS}$ & $\dagger 3.91 \mathrm{E}-29$ & † 933. & $\dagger 2.66 \mathrm{E}-24$ & $\dagger 994$. & $\dagger 5.07 \mathrm{E}-22$ & $\dagger 1107$. & $\dagger 1.25 \mathrm{E}-20$ & $\dagger 1180$ & $6.02 \mathrm{E}-20$ & 1237. & $4.97 \mathrm{E}-19$ & 1388. \\
\hline DI+SPEX & $\dagger 7.04 \mathrm{E}-25$ & $\dagger 99.0$ & $2.66 \mathrm{E}-20$ & 123. & $7.85 \mathrm{E}-19$ & 162. & $6.03 \mathrm{E}-18$ & 189. & $1.75 \mathrm{E}-17$ & 215. & $5.33 \mathrm{E}-17$ & 263. \\
\hline DI+DPEX & $+3.91 \mathrm{E}-28$ & +586. & $\dagger 2.66 \mathrm{E}-23$ & $\dagger 672$. & $9.38 \mathrm{E}-21$ & 740. & $7.94 \mathrm{E}-20$ & 765. & $2.21 \mathrm{E}-19$ & 781. & $2.11 \mathrm{E}-18$ & 859. \\
\hline DI SUM & $\dagger 1.00 \mathrm{E}-24$ & & $3.66 \mathrm{E}-20$ & & $4.59 \mathrm{E}-18$ & & $4.69 \mathrm{E}-17$ & & $1.42 \mathrm{E}-16$ & & $3.38 \mathrm{E}-16$ & \\
\hline TI & $1.66 \mathrm{E}-20$ & -5.30 & $2.83 \mathrm{E}-18$ & 1.89 & $1.01 \mathrm{E}-16$ & 37.0 & $2.29 \mathrm{E}-16$ & 66.6 & $2.40 \mathrm{E}-16$ & 95.6 & $5.18 \mathrm{E}-17$ & 191. \\
\hline $\mathrm{TI}+\mathrm{SS}$ & $\dagger 2.31 \mathrm{E}-21$ & $\dagger 144$ & $9.39 \mathrm{E}-19$ & 162. & $1.51 \mathrm{E}-17$ & 209. & $3.73 \mathrm{E}-17$ & 245. & $4.87 \mathrm{E}-17$ & 281. & $2.55 \mathrm{E}-17$ & 409. \\
\hline $\mathrm{TI}+\mathrm{DS}$ & $\dagger 4.62 \mathrm{E}-25$ & $\dagger 892$. & $\dagger 6.96 \mathrm{E}-22$ & $\dagger 944$. & $\dagger 1.56 \mathrm{E}-20$ & $\dagger 1064$ & $\dagger 8.39 \mathrm{E}-20$ & $\dagger 1144$. & $1.92 \mathrm{E}-19$ & 1207. & $3.37 \mathrm{E}-19$ & 1405. \\
\hline TI+SPEX & $3.08 \mathrm{E}-20$ & 58.0 & $4.62 \mathrm{E}-18$ & 73.0 & $2.03 \mathrm{E}-17$ & 120. & $4.45 \mathrm{E}-17$ & 153. & $5.64 \mathrm{E}-17$ & 184. & $2.79 \mathrm{E}-17$ & 280. \\
\hline TI+DPEX & $+2.31 \mathrm{E}-23$ & +545. & $\dagger 6.96 \mathrm{E}-21$ & $\dagger 622$. & $1.70 \mathrm{E}-19$ & 698. & $5.50 \mathrm{E}-19$ & 729. & $7.22 \mathrm{E}-19$ & 751. & $1.41 \mathrm{E}-18$ & 876. \\
\hline TI SUM & $4.97 \mathrm{E}-20$ & & $8.40 \mathrm{E}-18$ & & $1.37 \mathrm{E}-16$ & & $3.11 \mathrm{E}-16$ & & $3.46 \mathrm{E}-16$ & & $1.07 \mathrm{E}-16$ & \\
\hline DCAI & $1.78 \mathrm{E}-15$ & 31.1 & $1.06 \mathrm{E}-15$ & 60.9 & $6.17 \mathrm{E}-16$ & 138. & $3.10 \mathrm{E}-16$ & 178. & $1.18 \mathrm{E}-16$ & 208. & $2.77 \mathrm{E}-18$ & 300. \\
\hline DCAI+SS & $2.85 \mathrm{E}-18$ & 181. & $2.39 \mathrm{E}-17$ & 221. & $6.71 \mathrm{E}-17$ & 310. & $4.87 \mathrm{E}-17$ & 357. & $2.74 \mathrm{E}-17$ & 393. & $2.15 \mathrm{E}-18$ & 518. \\
\hline DCAI+DS & $\dagger 8.83 \mathrm{E}-20$ & † 929 & $\dagger 1.12 \mathrm{E}-19$ & $\dagger 1003$. & $\dagger 9.40 \mathrm{E}-20$ & $\dagger 1165$. & $\dagger 1.15 \mathrm{E}-19$ & $\dagger 1255$. & $1.04 \mathrm{E}-19$ & 1319. & $3.45 \mathrm{E}-20$ & 1514. \\
\hline DCAI+SPEX & $7.25 \mathrm{E}-17$ & 94.4 & $1.90 \mathrm{E}-16$ & 132. & $1.01 \mathrm{E}-16$ & 222. & $5.97 \mathrm{E}-17$ & 264. & $3.20 \mathrm{E}-17$ & 297. & $2.33 \mathrm{E}-18$ & 389. \\
\hline DCAI+DPEX & $\dagger 8.83 \mathrm{E}-19$ & $\dagger 581$. & $\dagger 1.12 \mathrm{E}-18$ & $\dagger 681$. & $6.13 \mathrm{E}-19$ & 799. & $5.73 \mathrm{E}-19$ & 840. & $3.90 \mathrm{E}-19$ & 863. & $1.36 \mathrm{E}-19$ & 985. \\
\hline DCAI SUM & $1.86 \mathrm{E}-15$ & & $1.28 \mathrm{E}-15$ & & $7.86 \mathrm{E}-16$ & & $4.19 \mathrm{E}-16$ & & $1.78 \mathrm{E}-16$ & & $7.42 \mathrm{E}-18$ & \\
\hline SC & $2.04 \mathrm{E}-15$ & -8.14 & $1.70 \mathrm{E}-15$ & -3.24 & $1.20 \mathrm{E}-15$ & 18.5 & $7.76 \mathrm{E}-16$ & 32.2 & $4.04 \mathrm{E}-16$ & 45.8 & $3.68 \mathrm{E}-17$ & 81.5 \\
\hline $\mathrm{SC}+\mathrm{SS}$ & $3.19 \mathrm{E}-19$ & 141. & $2.67 \mathrm{E}-18$ & 157. & $6.45 \mathrm{E}-18$ & 190. & $7.76 \mathrm{E}-18$ & 211. & 7.62E-18 & 231. & $3.69 \mathrm{E}-18$ & 299. \\
\hline $\mathrm{SC}+\mathrm{DS}$ & $\dagger 9.81 \mathrm{E}-22$ & $\dagger 889$ & $\dagger 1.58 \mathrm{E}-21$ & $\dagger 939$. & $\dagger 1.65 \mathrm{E}-21$ & $\dagger 1046$ & $\dagger 2.39 \mathrm{E}-21$ & $\dagger 1110$ & $3.41 \mathrm{E}-21$ & 1157. & $7.09 \mathrm{E}-21$ & 1296. \\
\hline SC+SPEX & $7.17 \mathrm{E}-18$ & 55.2 & $2.80 \mathrm{E}-17$ & 67.9 & $1.34 \mathrm{E}-17$ & 101. & $1.17 \mathrm{E}-17$ & 118. & $1.06 \mathrm{E}-17$ & 134. & $4.47 \mathrm{E}-18$ & 170. \\
\hline SC+DPEX & $\dagger 3.92 \mathrm{E}-21$ & $\dagger 542$. & $\dagger 7.89 \mathrm{E}-21$ & $\dagger 617$. & $9.54 \mathrm{E}-21$ & 680. & $2.26 \mathrm{E}-20$ & 694. & $1.11 \mathrm{E}-20$ & 701. & $3.77 \mathrm{E}-20$ & 767. \\
\hline SC SUM & $2.05 \mathrm{E}-15$ & & $1.73 \mathrm{E}-15$ & & $1.22 \mathrm{E}-15$ & & $7.95 \mathrm{E}-16$ & & $4.22 \mathrm{E}-16$ & & $4.50 \mathrm{E}-17$ & \\
\hline DC & $4.37 \mathrm{E}-17$ & -6.68 & $4.67 \mathrm{E}-17$ & 3.12 & $2.66 \mathrm{E}-17$ & 46.7 & $1.19 \mathrm{E}-17$ & 73.9 & $3.12 \mathrm{E}-18$ & 101. & $1.67 \mathrm{E}-19$ & 179. \\
\hline $\mathrm{DC}+\mathrm{SS}$ & $2.42 \mathrm{E}-19$ & 143. & $3.98 \mathrm{E}-18$ & 163. & $1.35 \mathrm{E}-17$ & 219. & $9.55 \mathrm{E}-18$ & 253. & $3.60 \mathrm{E}-18$ & 286. & $4.99 \mathrm{E}-19$ & 397. \\
\hline $\mathrm{DC}+\mathrm{DS}$ & $\dagger 7.24 \mathrm{E}-21$ & $\dagger 891$ & $\dagger 2.89 \mathrm{E}-20$ & $\dagger 946$. & $\dagger 2.59 \mathrm{E}-20$ & $\dagger 1074$ & $\dagger 2.61 \mathrm{E}-20$ & $\dagger 1151$. & $1.85 \mathrm{E}-20$ & 1212. & $1.03 \mathrm{E}-20$ & 1393. \\
\hline $\mathrm{DC}+$ SPEX & $7.75 \mathrm{E}-18$ & 56.6 & $3.52 \mathrm{E}-17$ & 74.2 & $1.90 \mathrm{E}-17$ & 130. & $1.11 \mathrm{E}-17$ & 160. & $3.99 \mathrm{E}-18$ & 190. & $5.23 \mathrm{E}-19$ & 268. \\
\hline DC+DPEX & $\dagger 3.62 \mathrm{E}-19$ & $\dagger 543$ & $\dagger 1.15 \mathrm{E}-19$ & $\dagger 623$. & $1.06 \mathrm{E}-19$ & 708. & $1.51 \mathrm{E}-19$ & 736. & $6.88 \mathrm{E}-20$ & 756. & $4.15 \mathrm{E}-20$ & 864. \\
\hline DC SUM & $5.21 \mathrm{E}-17$ & & $8.60 \mathrm{E}-17$ & & $5.92 \mathrm{E}-17$ & & $3.27 \mathrm{E}-17$ & & $1.08 \mathrm{E}-17$ & & $1.24 \mathrm{E}-18$ & \\
\hline TEX & $5.67 \mathrm{E}-19$ & 7.70 & $1.77 \mathrm{E}-17$ & 7.70 & $1.73 \mathrm{E}-16$ & 7.70 & $2.84 \mathrm{E}-16$ & 7.70 & $3.86 \mathrm{E}-16$ & 7.70 & $6.09 \mathrm{E}-16$ & 7.70 \\
\hline TEX+SS & $9.08 \mathrm{E}-21$ & 157. & $3.41 \mathrm{E}-19$ & 168. & $4.92 \mathrm{E}-19$ & 180. & $1.13 \mathrm{E}-18$ & 186. & $2.04 \mathrm{E}-18$ & 193. & $4.17 \mathrm{E}-18$ & 225. \\
\hline TEX+DS & $\dagger 3.65 E-25$ & $\dagger 905$. & $\dagger 1.88 \mathrm{E}-23$ & $\dagger 950$. & $\dagger 2.50 \mathrm{E}-22$ & $\dagger 1035$. & $\dagger 9.09 \mathrm{E}-22$ & $\dagger 1085$. & $\dagger 2.33 \mathrm{E}-21$ & $\dagger 1119$ & $8.00 \mathrm{E}-21$ & 1222. \\
\hline TEX+SPEX & $1.95 \mathrm{E}-19$ & 71.0 & $2.92 \mathrm{E}-18$ & 78.8 & $1.24 \mathrm{E}-18$ & 90.5 & $1.95 \mathrm{E}-18$ & 93.9 & $3.03 \mathrm{E}-18$ & 96.2 & $5.68 \mathrm{E}-18$ & 96.2 \\
\hline TEX+DPEX & $\dagger 7.37 \mathrm{E}-25$ & $\dagger 558$. & $\dagger 3.76 \mathrm{E}-23$ & $\dagger 628$. & $\dagger 6.26 \mathrm{E}-22$ & $\dagger 669$. & $\dagger 1.82 \mathrm{E}-21$ & $\dagger 670$. & $\dagger 4.66 \mathrm{E}-21$ & $\dagger 663$ & $2.40 \mathrm{E}-20$ & 693. \\
\hline TEX SUM & $7.71 \mathrm{E}-19$ & & $\S 2.10 \mathrm{E}-17$ & & $1.75 \mathrm{E}-16$ & & $2.87 \mathrm{E}-16$ & & $3.91 \mathrm{E}-16$ & & $6.19 \mathrm{E}-16$ & \\
\hline \multirow[t]{4}{*}{ Channel } & Energy & & & & & & & & & & & \\
\hline & $500 \mathrm{keV} / \mathrm{u}$ & & 1000 & & 2000 & & 5000 & & 10000 & & 25000 & \\
\hline & cross & & cross & & cross & & cross & & cross & & cross & \\
\hline & $\begin{array}{l}\text { section } \\
\mathrm{cm}^{2}\end{array}$ & $\begin{array}{l}\text { Eloss } \\
\text { eV }\end{array}$ & $\begin{array}{l}\text { section } \\
\mathrm{cm}^{2}\end{array}$ & $\begin{array}{l}\text { Eloss } \\
\mathrm{eV}\end{array}$ & $\begin{array}{l}\text { section } \\
\mathrm{cm}^{2}\end{array}$ & $\begin{array}{l}\text { Eloss } \\
\mathrm{eV}\end{array}$ & $\begin{array}{l}\text { section } \\
\mathrm{cm}^{2}\end{array}$ & $\begin{array}{l}\text { Eloss } \\
\mathrm{eV}\end{array}$ & $\begin{array}{l}\text { section } \\
\mathrm{cm}^{2}\end{array}$ & $\begin{array}{l}\text { Eloss } \\
\mathrm{eV}\end{array}$ & $\begin{array}{l}\text { section } \\
\mathrm{cm}^{2}\end{array}$ & Eloss \\
\hline SI & $* 1.06 \mathrm{E}-15$ & $* 39.6$ & $* 6.19 \mathrm{E}-16$ & $* 46.9$ & $* 3.32 \mathrm{E}-16$ & $* 56.4$ & $* 1.30 \mathrm{E}-16$ & ${ }^{*} 69.1$ & $* 6.29 \mathrm{E}-17$ & $* 76.8$ & $* 2.47 \mathrm{E}-17$ & $* 83.1$ \\
\hline $\mathrm{SI}+\mathrm{SS}$ & ${ }^{*} 5.46 \mathrm{E}-17$ & $* 333$ & * 5.84E-17 & * 401 . & $* 5.51 \mathrm{E}-17$ & * 465 . & * $4.20 \mathrm{E}-17$ & * 539 . & $* 3.01 \mathrm{E}-17$ & * 580. & ${ }^{*} 1.4 / \mathrm{E}-1 /$ & * 635.1. \\
\hline SI+DS & ${ }^{*} 1.75 \mathrm{E}-18$ & * 1304. & ${ }^{*} 1.89 \mathrm{E}-18$ & ${ }^{*} 1553$. & ${ }^{*} 1.82 \mathrm{E}-18$ & * 1940. & ${ }^{*} 1.65 \mathrm{E}-18$ & $* 2509$. & ${ }^{*} 1.16 \mathrm{E}-18$ & * 2914. & $* 7.24 \mathrm{E}-19$ & * 3427 \\
\hline SI+SPEX & ${ }^{*} 6.46 \mathrm{E}-17$ & $* 126$. & $* 6.46 \mathrm{E}-17$ & $* 132$. & $* 5.77 \mathrm{E}-17$ & ${ }^{*} 141$ & $* 4.44 \mathrm{E}-17$ & * 153. & $* 3.05 \mathrm{E}-17$ & $* 160$ & $* 1.68 \mathrm{E}-17$ & $* 166$ \\
\hline SI+DPEX & $* 2.12 \mathrm{E}-18$ & * 798. & ${ }^{*} 2.58 \mathrm{E}-18$ & * 836 & * $3.17 \mathrm{E}-18$ & $* 850$ & ${ }^{*} 2.47 \mathrm{E}-18$ & $* 848$ & ${ }^{*} 1.38 \mathrm{E}-18$ & * 867 & $* 8.65 \mathrm{E}-19$ & $* 871$. \\
\hline SI SUM & ${ }^{*} 1.18 \mathrm{E}-15$ & & $* 7.46 \mathrm{E}-16$ & & ${ }^{*} 4.50 \mathrm{E}-16$ & & $* 2.21 \mathrm{E}-16$ & & ${ }^{*} 1.26 \mathrm{E}-16$ & & ${ }^{*} 5.99 \mathrm{E}-17$ & \\
\hline DI & $1.20 \mathrm{E}-16$ & 219. & $5.11 \mathrm{E}-17$ & 243. & $1.87 \mathrm{E}-17$ & 261. & $3.89 \mathrm{E}-18$ & 273. & $1.14 \mathrm{E}-18$ & 277. & $2.10 \mathrm{E}-19$ & 267. \\
\hline $\mathrm{DI}+\mathrm{SS}$ & $4.34 \mathrm{E}-17$ & 512. & $2.52 \mathrm{E}-17$ & 597. & $1.29 \mathrm{E}-17$ & 669. & $4.62 \mathrm{E}-18$ & 743. & $1.96 \mathrm{E}-18$ & 780. & $5.49 \mathrm{E}-19$ & 819. \\
\hline $\mathrm{DI}+\mathrm{DS}$ & $3.49 \mathrm{E}-18$ & 1483. & $1.46 \mathrm{E}-18$ & 1749. & $6.25 \mathrm{E}-19$ & 2144. & $2.44 \mathrm{E}-19$ & 2713. & $1.40 \mathrm{E}-19$ & 3115. & $3.23 \mathrm{E}-20$ & 3611. \\
\hline DI+SPEX & $4.77 \mathrm{E}-17$ & 306. & $2.68 \mathrm{E}-17$ & 328. & $1.33 \mathrm{E}-17$ & 346. & $4.79 \mathrm{E}-18$ & 357. & $1.97 \mathrm{E}-18$ & 360. & $5.49 \mathrm{E}-19$ & 350. \\
\hline DI+DPEX & $4.12 \mathrm{E}-18$ & 977. & $2.36 \mathrm{E}-18$ & 1032. & $1.07 \mathrm{E}-18$ & 1055. & $3.47 \mathrm{E}-19$ & 1052. & $1.23 \mathrm{E}-19$ & 1067. & $3.86 \mathrm{E}-20$ & 1055. \\
\hline DI SUM & $2.19 \mathrm{E}-16$ & & $1.07 \mathrm{E}-16$ & & $4.66 \mathrm{E}-17$ & & $1.39 \mathrm{E}-17$ & & $5.30 \mathrm{E}-18$ & & $1.38 \mathrm{E}-18$ & \\
\hline $\mathrm{TI}$ & $7.76 \mathrm{E}-19$ & 398. & $3.42 \mathrm{E}-20$ & 809. & $2.24 \mathrm{E}-21$ & 1166. & $5.28 \mathrm{E}-23$ & 2802. & $\dagger 3.00 \mathrm{E}-24$ & $\dagger 5431$. & $+6.50 \mathrm{E}-26$ & $\dagger 13544$. \\
\hline $\mathrm{TI}+\mathrm{SS}$ & $1.60 \mathrm{E}-18$ & 691. & $9.37 \mathrm{E}-20$ & 1163. & $4.97 \mathrm{E}-21$ & 1574. & $1.09 \mathrm{E}-22$ & 3272. & $5.02 \mathrm{E}-24$ & 5934. & $1.04 \mathrm{E}-25$ & 14096. \\
\hline $\mathrm{TI}+\mathrm{DS}$ & $1.86 \mathrm{E}-19$ & 1662. & $8.49 \mathrm{E}-21$ & 2315. & $3.82 \mathrm{E}-22$ & 3049. & $6.79 \mathrm{E}-24$ & 5242. & $2.64 \mathrm{E}-25$ & 8269. & $2.08 \mathrm{E}-26$ & 16888. \\
\hline TI+SPEX & $1.67 \mathrm{E}-18$ & 485. & $9.51 \mathrm{E}-20$ & 894. & $5.00 \mathrm{E}-21$ & 1251. & $1.15 \mathrm{E}-22$ & 2886 . & $5.02 \mathrm{E}-24$ & 5514. & $1.04 \mathrm{E}-25$ & 13627. \\
\hline TI+DPEX & $2.21 \mathrm{E}-19$ & 1156. & $1.35 \mathrm{E}-20$ & 1598. & $6.08 \mathrm{E}-22$ & 1960. & $8.48 \mathrm{E}-24$ & 3581. & $7.93 \mathrm{E}-25$ & 6221. & $2.08 \mathrm{E}-26$ & 14332. \\
\hline TI SUM & $4.45 \mathrm{E}-18$ & & $2.45 \mathrm{E}-19$ & & $1.32 \mathrm{E}-20$ & & $2.92 \mathrm{E}-22$ & & $1.41 \mathrm{E}-23$ & & $3.15 \mathrm{E}-25$ & \\
\hline
\end{tabular}


Table 8 (continued)

\begin{tabular}{|c|c|c|c|c|c|c|c|c|c|c|c|c|}
\hline DCAI & $9.08 \mathrm{E}-22$ & $\ddagger 523$. & $+1.00 \mathrm{E}-25$ & $\ddagger 872$. & $\dagger 1.00 \mathrm{E}-29$ & +1500 & $\dagger 1.00 \mathrm{E}-34$ & +2300 & $\dagger 1.50 \mathrm{E}-38$ & $\dagger 3500$ & $+1.50 \mathrm{E}-43$ & $\dagger 6400$ \\
\hline $\mathrm{DCAI}+\mathrm{SS}$ & $5.80 \mathrm{E}-21$ & 816. & $2.45 \mathrm{E}-23$ & 1226. & $4.95 \mathrm{E}-26$ & 1908. & $1.25 \mathrm{E}-29$ & 2770 . & $2.50 \mathrm{E}-32$ & 4003. & $5.00 \mathrm{E}-36$ & 6952. \\
\hline $\mathrm{DCAI}+\mathrm{DS}$ & $8.35 \mathrm{E}-22$ & 1787. & $5.33 \mathrm{E}-25$ & 2378. & $4.95 \mathrm{E}-28$ & 3383. & $1.25 \mathrm{E}-32$ & 4740. & $2.50 \mathrm{E}-36$ & 6338. & $5.00 \mathrm{E}-41$ & 9744. \\
\hline DCAI+SPEX & $5.95 \mathrm{E}-21$ & 610. & $2.45 \mathrm{E}-23$ & 957. & $4.95 \mathrm{E}-26$ & 1585. & $1.25 \mathrm{E}-29$ & 2384. & $2.50 \mathrm{E}-32$ & 3583. & $5.00 \mathrm{E}-36$ & 6483. \\
\hline DCAI+DPEX & $1.01 \mathrm{E}-21$ & 1281. & $1.07 \mathrm{E}-24$ & 1661. & $4.95 \mathrm{E}-28$ & 2294. & $1.25 \mathrm{E}-32$ & 3079. & $2.50 \mathrm{E}-36$ & 4290. & $5.00 \mathrm{E}-41$ & 7188. \\
\hline DCAI SUM & $\ddagger 1.45 \mathrm{E}-20$ & & $\ddagger 5.07 \mathrm{E}-23$ & & $1.00 \mathrm{E}-25$ & & $2.50 \mathrm{E}-29$ & & $5.00 \mathrm{E}-32$ & & $1.00 \mathrm{E}-35$ & \\
\hline SC & $3.64 \mathrm{E}-19$ & 245. & $3.24 \mathrm{E}-21$ & 464. & $\dagger 1.00 \mathrm{E}-23$ & $\dagger 1008$ & $\dagger 1.00 \mathrm{E}-28$ & +2642. & $\dagger 8.00 \mathrm{E}-32$ & $\dagger 5365$. & $+7.00 \mathrm{E}-36$ & $\dagger 13534$. \\
\hline $\mathrm{SC}+\mathrm{SS}$ & $4.80 \mathrm{E}-19$ & 538. & $4.72 \mathrm{E}-20$ & 818. & $4.78 \mathrm{E}-21$ & 1416. & $1.47 \mathrm{E}-22$ & 3112. & $1.05 \mathrm{E}-23$ & 5868. & $2.56 \mathrm{E}-25$ & 14086. \\
\hline $\mathrm{SC}+\mathrm{DS}$ & $2.23 \mathrm{E}-20$ & 1509. & $2.80 \mathrm{E}-21$ & 1970. & $1.76 \mathrm{E}-22$ & 2891. & $6.02 \mathrm{E}-24$ & 5802. & $2.83 \mathrm{E}-25$ & 8203. & $2.56 \mathrm{E}-27$ & 16878. \\
\hline SC+SPEX & $5.27 \mathrm{E}-19$ & 332. & $4.81 \mathrm{E}-20$ & 549. & $4.78 \mathrm{E}-21$ & 1093. & $1.47 \mathrm{E}-22$ & 2726 . & $1.05 \mathrm{E}-23$ & 5448. & $2.56 \mathrm{E}-25$ & 13617. \\
\hline SC+DPEX & $2.70 \mathrm{E}-20$ & 1003. & $4.69 \mathrm{E}-21$ & 1253. & $3.60 \mathrm{E}-22$ & 1802. & $9.04 \mathrm{E}-24$ & 3421. & $2.83 \mathrm{E}-25$ & 6155. & $2.56 \mathrm{E}-27$ & 14322. \\
\hline SC SUM & $1.42 \mathrm{E}-18$ & & $1.06 \mathrm{E}-19$ & & $1.01 \mathrm{E}-20$ & & $3.09 \mathrm{E}-22$ & & $2.16 \mathrm{E}-23$ & & $5.17 \mathrm{E}-25$ & \\
\hline $\mathrm{DC}$ & $1.99 \mathrm{E}-22$ & 506. & $\dagger 1.00 \mathrm{E}-25$ & $\dagger 963$. & $\dagger 5.00 \mathrm{E}-29$ & $\dagger 2052$ & $\dagger 2.00 \mathrm{E}-33$ & $\dagger 5320$. & $\dagger 1.00 \mathrm{E}-36$ & $\dagger 10766$ & $\dagger 5.00 \mathrm{E}-41$ & $\dagger 27104$ \\
\hline $\mathrm{DC}+\mathrm{SS}$ & $3.79 \mathrm{E}-21$ & 799. & $4.66 \mathrm{E}-23$ & 1317. & $4.22 \mathrm{E}-25$ & 2460. & $2.50 \mathrm{E}-28$ & 5790. & $1.00 \mathrm{E}-30$ & 11269. & $7.50 \mathrm{E}-34$ & 27656. \\
\hline $\mathrm{DC}+\mathrm{DS}$ & $6.59 \mathrm{E}-22$ & 1770. & $6.57 \mathrm{E}-24$ & 2469. & $6.33 \mathrm{E}-26$ & 3935. & $2.50 \mathrm{E}-31$ & 7760. & $1.00 \mathrm{E}-34$ & 13604. & $7.50 \mathrm{E}-39$ & 30448. \\
\hline $\mathrm{DC}+\mathrm{SPEX}$ & $3.85 \mathrm{E}-21$ & 593. & $4.66 \mathrm{E}-23$ & 1048. & $4.22 \mathrm{E}-25$ & 2137. & $2.50 \mathrm{E}-28$ & 5404. & $1.00 \mathrm{E}-30$ & 10849. & $7.50 \mathrm{E}-34$ & 27187. \\
\hline DC+DPEX & $7.59 \mathrm{E}-22$ & 1264. & $1.03 \mathrm{E}-23$ & 1752. & $9.28 \mathrm{E}-26$ & 2846 . & $2.50 \mathrm{E}-31$ & 6099. & $1.00 \mathrm{E}-34$ & 11556. & $7.50 \mathrm{E}-39$ & 27892 . \\
\hline DC SUM & $9.26 \mathrm{E}-21$ & & $1.10 \mathrm{E}-22$ & & $1.00 \mathrm{E}-24$ & & $5.01 \mathrm{E}-28$ & & $2.00 \mathrm{E}-30$ & & $1.50 \mathrm{E}-33$ & \\
\hline TEX & $6.97 \mathrm{E}-16$ & 7.70 & $5.52 \mathrm{E}-16$ & 7.70 & $3.40 \mathrm{E}-16$ & 7.70 & $1.58 \mathrm{E}-16$ & 7.70 & $7.39 \mathrm{E}-17$ & 7.70 & $2.41 \mathrm{E}-17$ & 7.70 \\
\hline TEX+SS & $1.17 \mathrm{E}-17$ & 301. & $1.60 \mathrm{E}-17$ & 362. & $1.78 \mathrm{E}-17$ & 416. & $1.70 \mathrm{E}-17$ & 478. & $1.42 \mathrm{E}-17$ & 511. & $9.45 \mathrm{E}-18$ & 560. \\
\hline TEX+DS & $3.65 \mathrm{E}-19$ & 1272. & $8.30 \mathrm{E}-19$ & 1514. & $1.15 \mathrm{E}-18$ & 1891. & $1.04 \mathrm{E}-18$ & 2448. & $6.95 \mathrm{E}-19$ & 2846. & $4.52 \mathrm{E}-19$ & 3351. \\
\hline TEX+SPEX & $1.39 \mathrm{E}-17$ & 94.2 & $1.78 \mathrm{E}-17$ & 93.0 & $1.87 \mathrm{E}-17$ & 92.6 & $1.82 \mathrm{E}-17$ & 91.8 & $1.44 \mathrm{E}-17$ & 91.0 & $9.45 \mathrm{E}-18$ & 91.0 \\
\hline TEX+DPEX & $4.04 \mathrm{E}-19$ & 766. & $1.06 \mathrm{E}-18$ & 797. & $1.51 \mathrm{E}-18$ & 802. & $1.27 \mathrm{E}-18$ & 787. & $7.61 \mathrm{E}-19$ & 798. & $4.95 \mathrm{E}-19$ & 796. \\
\hline TEX SUM & $7.23 \mathrm{E}-16$ & & $5.88 \mathrm{E}-16$ & & $3.79 \mathrm{E}-16$ & & $1.96 \mathrm{E}-16$ & & $1.04 \mathrm{E}-16$ & & $4.40 \mathrm{E}-17$ & \\
\hline
\end{tabular}

$\uparrow$ Extrapolated. $\ddagger$ Significantly improved. § Corrects typographical error in Ref. [1]. ${ }^{*}$ Includes Born correction. 
Table 9

The integral cross section and associated average energy loss for NSIM and SIM processes in $\mathrm{O}^{6+}+\mathrm{H}_{2}$ collisions with impact energies between 1 and 25000 keV/u.

\begin{tabular}{|c|c|c|c|c|c|c|c|c|c|c|c|c|}
\hline \multirow[t]{2}{*}{ Channel } & $\begin{array}{l}\text { Energy } \\
1 \mathrm{keV} / \mathrm{u}\end{array}$ & & 10 & & 50 & & 75 & & 100 & & 200 & \\
\hline & $\begin{array}{l}\text { cross } \\
\text { section } \\
\mathrm{cm}^{2}\end{array}$ & $\begin{array}{l}\text { Eloss } \\
\mathrm{eV}\end{array}$ & $\begin{array}{l}\text { cross } \\
\text { section } \\
\mathrm{cm}^{2}\end{array}$ & $\begin{array}{l}\text { Eloss } \\
\text { eV }\end{array}$ & $\begin{array}{l}\text { cross } \\
\text { section } \\
\mathrm{cm}^{2}\end{array}$ & $\begin{array}{l}\text { Eloss } \\
\mathrm{eV}\end{array}$ & $\begin{array}{l}\text { cross } \\
\text { section } \\
\mathrm{cm}^{2}\end{array}$ & $\begin{array}{l}\text { Eloss } \\
\mathrm{eV}\end{array}$ & $\begin{array}{l}\text { cross } \\
\text { section } \\
\mathrm{cm}^{2}\end{array}$ & $\begin{array}{l}\text { Eloss } \\
\mathrm{eV}\end{array}$ & $\begin{array}{l}\text { cross } \\
\text { section } \\
\mathrm{cm}^{2}\end{array}$ & $\begin{array}{l}\text { Eloss } \\
\mathrm{eV}\end{array}$ \\
\hline $\begin{array}{l}\text { SI } \\
\text { SI+SS } \\
\text { SI+DS } \\
\text { SI+SPEX } \\
\text { SI+DPEX } \\
\text { SI SUM }\end{array}$ & $\begin{array}{l}3.27 \mathrm{E}-20 \\
+1.67 \mathrm{E}-24 \\
+2.67 \mathrm{E}-26 \\
+6.66 \mathrm{E}-22 \\
+3.33 \mathrm{E}-28 \\
3.34 \mathrm{E}-20\end{array}$ & $\begin{array}{l}17.1 \\
\dagger 846 . \\
\dagger 1708 \\
\dagger 437 \\
\dagger 817\end{array}$ & $\begin{array}{l}2.67 \mathrm{E}-18 \\
\dagger 1.36 \mathrm{E}-22 \\
+5.45 \mathrm{E}-23 \\
5.91 \mathrm{E}-20 \\
\dagger 2.72 \mathrm{E}-25 \\
2.73 \mathrm{E}-18\end{array}$ & $\begin{array}{l}23.0 \\
\dagger 862 . \\
\dagger 1834 . \\
492 . \\
+923\end{array}$ & $\begin{array}{l}* 4.16 \mathrm{E}-16 \\
* 1.93 \mathrm{E}-20 \\
*+3.19 \mathrm{E}-21 \\
* 1.35 \mathrm{E}-19 \\
*+7.08 \mathrm{E}-22 \\
* 4.16 \mathrm{E}-16\end{array}$ & $\begin{array}{l}* 20.5 \\
* 879 . \\
* \dagger 2031 . \\
500 . \\
* \dagger 1021 .\end{array}$ & $\begin{array}{l}* 8.95 \mathrm{E}-16 \\
* 4.74 \mathrm{E}-20 \\
* \dagger 1.21 \mathrm{E}-20 \\
* 5.88 \mathrm{E}-19 \\
* 6.27 \mathrm{E}-21 \\
* 8.96 \mathrm{E}-16\end{array}$ & $\begin{array}{l}* 27.9 \\
* 892 . \\
*+2089 . \\
* 518 \\
* 1330\end{array}$ & $\begin{array}{l}* 1.51 \mathrm{E}-15 \\
* 1.72 \mathrm{E}-19 \\
* 2.88 \mathrm{E}-20 \\
* 8.73 \mathrm{E}-19 \\
* 7.76 \mathrm{E}-20 \\
* 1.51 \mathrm{E}-15\end{array}$ & $\begin{array}{l}* 33.6 \\
* 931 \\
* 2142 \\
* 531 \\
* 1149\end{array}$ & $\begin{array}{l}* 2.45 \mathrm{E}-15 \\
* 9.07 \mathrm{E}-19 \\
* 1.10 \mathrm{E}-19 \\
* 1.73 \mathrm{E}-18 \\
* 4.11 \mathrm{E}-19 \\
* 2.45 \mathrm{E}-15\end{array}$ & $\begin{array}{l}* 34.4 \\
* 950 . \\
* 2194 . \\
* 549 . \\
* 1217\end{array}$ \\
\hline $\begin{array}{l}\text { DI } \\
\text { DI+SS } \\
\text { DI+DS } \\
\text { DI+SPEX } \\
\text { DI+DPEX } \\
\text { DI SUM }\end{array}$ & $\begin{array}{l}+5.00 \mathrm{E}-23 \\
+1.66 \mathrm{E}-28 \\
+1.66 \mathrm{E}-28 \\
+1.66 \mathrm{E}-30 \\
+1.66 \mathrm{E}-28 \\
+5.00 \mathrm{E}-23\end{array}$ & $\begin{array}{l}\dagger 37.7 \\
\dagger 867 . \\
\dagger 1728 \\
\dagger 458 \\
\dagger 838\end{array}$ & $\begin{array}{l}7.42 \mathrm{E}-19 \\
\dagger 5.30 \mathrm{E}-23 \\
\dagger 1.06 \mathrm{E}-23 \\
\dagger 5.26 \mathrm{E}-25 \\
\dagger 1.06 \mathrm{E}-23 \\
7.42 \mathrm{E}-19\end{array}$ & $\begin{array}{l}55.7 \\
\dagger 895 . \\
\dagger 1866 \\
\dagger 525 . \\
\dagger 956\end{array}$ & $\begin{array}{l}2.30 \mathrm{E}-18 \\
8.43 \mathrm{E}-22 \\
\dagger 1.06 \mathrm{E}-22 \\
1.18 \mathrm{E}-20 \\
\dagger 1.06 \mathrm{E}-20 \\
2.32 \mathrm{E}-18\end{array}$ & $\begin{array}{l}66.3 \\
925 . \\
\dagger 2077 \\
545 \\
\dagger 1066\end{array}$ & $\begin{array}{l}3.16 \mathrm{E}-17 \\
1.96 \mathrm{E}-20 \\
+2.78 \mathrm{E}-21 \\
1.74 \mathrm{E}-19 \\
2.64 \mathrm{E}-21 \\
3.18 \mathrm{E}-17\end{array}$ & $\begin{array}{l}91.4 \\
956 . \\
\dagger 2152 . \\
581 . \\
1393 .\end{array}$ & $\begin{array}{l}1.28 \mathrm{E}-16 \\
1.17 \mathrm{E}-19 \\
2.09 \mathrm{E}-20 \\
5.45 \mathrm{E}-19 \\
5.54 \mathrm{E}-20 \\
1.29 \mathrm{E}-16\end{array}$ & $\begin{array}{l}117 . \\
1014 . \\
2226 . \\
614 . \\
1232 .\end{array}$ & $\begin{array}{l}4.12 \mathrm{E}-16 \\
1.54 \mathrm{E}-18 \\
1.88 \mathrm{E}-19 \\
2.88 \mathrm{E}-18 \\
7.13 \mathrm{E}-19 \\
4.17 \mathrm{E}-16\end{array}$ & $\begin{array}{l}166 . \\
1081 . \\
2326 . \\
681 . \\
1349 .\end{array}$ \\
\hline $\begin{array}{l}\text { TI } \\
\text { TI+SS } \\
\text { TI+DS } \\
\text { TI+SPEX } \\
\text { TI+DPEX } \\
\text { TI SUM }\end{array}$ & $\begin{array}{l}2.21 \mathrm{E}-20 \\
+2.20 \mathrm{E}-24 \\
+2.20 \mathrm{E}-25 \\
+2.20 \mathrm{E}-22 \\
+2.20 \mathrm{E}-25 \\
2.23 \mathrm{E}-20\end{array}$ & $\begin{array}{l}1.90 \\
\dagger 831 . \\
\dagger 1693 . \\
\dagger 422 \\
\dagger 802\end{array}$ & $\begin{array}{l}6.51 \mathrm{E}-18 \\
\dagger 1.31 \mathrm{E}-21 \\
\dagger 1.31 \mathrm{E}-22 \\
1.30 \mathrm{E}-19 \\
\dagger 1.31 \mathrm{E}-22 \\
6.64 \mathrm{E}-18\end{array}$ & $\begin{array}{l}12.7 \\
\dagger 852 . \\
\dagger 1823 . \\
482 . \\
\dagger 913\end{array}$ & $\begin{array}{l}1.10 \mathrm{E}-16 \\
4.40 \mathrm{E}-20 \\
4.88 \mathrm{E}-21 \\
\dagger 4.26 \mathrm{E}-19 \\
4.88 \mathrm{E}-21 \\
1.10 \mathrm{E}-16\end{array}$ & $\begin{array}{l}44.2 \\
\dagger 903 . \\
2055 . \\
\dagger 523 . \\
1044 .\end{array}$ & $\begin{array}{l}3.19 \mathrm{E}-16 \\
1.89 \mathrm{E}-19 \\
\dagger 2.86 \mathrm{E}-20 \\
2.03 \mathrm{E}-18 \\
3.37 \mathrm{E}-20 \\
3.21 \mathrm{E}-16\end{array}$ & $\begin{array}{l}72.8 \\
937 . \\
\dagger 2134 . \\
563 . \\
1375 .\end{array}$ & $\begin{array}{l}4.24 \mathrm{E}-16 \\
5.55 \mathrm{E}-19 \\
7.98 \mathrm{E}-20 \\
2.75 \mathrm{E}-18 \\
2.41 \mathrm{E}-19 \\
4.28 \mathrm{E}-16\end{array}$ & $\begin{array}{l}102 . \\
999 . \\
2211 . \\
599 . \\
1217 .\end{array}$ & $\begin{array}{l}1.55 \mathrm{E}-16 \\
1.35 \mathrm{E}-18 \\
1.75 \mathrm{E}-19 \\
2.49 \mathrm{E}-18 \\
6.31 \mathrm{E}-19 \\
1.60 \mathrm{E}-16\end{array}$ & $\begin{array}{l}172 . \\
1087 . \\
3247 . \\
1602 . \\
2270 .\end{array}$ \\
\hline $\begin{array}{l}\text { DCAI } \\
\text { DCAI+SS } \\
\text { DCAI+DS } \\
\text { DCAI+SPEX } \\
\text { DCAI+DPEX } \\
\text { DCAI SUM }\end{array}$ & $\begin{array}{l}2.35 \mathrm{E}-15 \\
+1.10 \mathrm{E}-19 \\
+2.20 \mathrm{E}-20 \\
+2.20 \mathrm{E}-18 \\
+2.20 \mathrm{E}-20 \\
2.35 \mathrm{E}-15\end{array}$ & $\begin{array}{l}47.4 \\
+877 \\
+1738 \\
+467 \\
+847\end{array}$ & $\begin{array}{l}1.78 \mathrm{E}-15 \\
\dagger 1.67 \mathrm{E}-19 \\
\dagger 3.34 \mathrm{E}-20 \\
3.54 \mathrm{E}-18 \\
\dagger 3.34 \mathrm{E}-20 \\
1.78 \mathrm{E}-15\end{array}$ & $\begin{array}{l}74.2 \\
\dagger 914 . \\
\dagger 1885 . \\
543 . \\
\dagger 974\end{array}$ & $\begin{array}{l}1.14 \mathrm{E}-15 \\
3.12 \mathrm{E}-19 \\
+5.01 \mathrm{E}-20 \\
2.97 \mathrm{E}-18 \\
+5.01 \mathrm{E}-20 \\
1.14 \mathrm{E}-15\end{array}$ & $\begin{array}{l}152 . \\
1010 . \\
\dagger 2163 . \\
631 . \\
\dagger 1152\end{array}$ & $\begin{array}{l}6.83 \mathrm{E}-16 \\
4.23 \mathrm{E}-19 \\
\dagger 6.10 \mathrm{E}-20 \\
4.26 \mathrm{E}-18 \\
6.16 \mathrm{E}-20 \\
6.88 \mathrm{E}-16\end{array}$ & $\begin{array}{l}197 . \\
1061 . \\
\dagger 2258 . \\
687 . \\
1499 .\end{array}$ & $\begin{array}{l}3.19 \mathrm{E}-16 \\
5.07 \mathrm{E}-19 \\
7.42 \mathrm{E}-20 \\
2.49 \mathrm{E}-18 \\
2.31 \mathrm{E}-19 \\
3.22 \mathrm{E}-16\end{array}$ & $\begin{array}{l}228 . \\
1125 . \\
2337 . \\
725 . \\
1343 .\end{array}$ & $\begin{array}{l}1.50 \mathrm{E}-17 \\
1.93 \mathrm{E}-19 \\
2.15 \mathrm{E}-20 \\
3.68 \mathrm{E}-19 \\
8.69 \mathrm{E}-20 \\
1.57 \mathrm{E}-17\end{array}$ & $\begin{array}{l}320 . \\
1235 . \\
2480 . \\
835 . \\
1503 .\end{array}$ \\
\hline $\begin{array}{l}\text { SC } \\
\text { SC+SS } \\
\text { SC+DS } \\
\text { SC+SPEX } \\
\text { SC+DPEX } \\
\text { SC SUM }\end{array}$ & $\begin{array}{l}2.33 \mathrm{E}-15 \\
+2.26 \mathrm{E}-21 \\
\dagger 1.13 \mathrm{E}-21 \\
\dagger 1.13 \mathrm{E}-19 \\
\dagger 2.26 \mathrm{E}-22 \\
2.33 \mathrm{E}-15\end{array}$ & $\begin{array}{l}-16.2 \\
\dagger 813 . \\
\dagger 1675 \\
\dagger 404 \\
\dagger 784\end{array}$ & $\begin{array}{l}1.95 \mathrm{E}-15 \\
\dagger 3.77 \mathrm{E}-21 \\
\dagger 1.89 \mathrm{E}-21 \\
1.79 \mathrm{E}-19 \\
\dagger 3.77 \mathrm{E}-22 \\
1.95 \mathrm{E}-15\end{array}$ & $\begin{array}{l}-11.3 \\
\dagger 828 . \\
\dagger 1799 . \\
458 . \\
+889\end{array}$ & $\begin{array}{l}1.48 \mathrm{E}-15 \\
\dagger 5.64 \mathrm{E}-21 \\
\dagger 2.25 \mathrm{E}-21 \\
2.70 \mathrm{E}-20 \\
\dagger 5.64 \mathrm{E}-22 \\
1.48 \mathrm{E}-15\end{array}$ & $\begin{array}{l}10.5 \\
\dagger 869 \\
+2021 \\
490 \\
+1011\end{array}$ & $\begin{array}{l}1.04 \mathrm{E}-15 \\
\dagger 7.25 \mathrm{E}-21 \\
\dagger 3.63 \mathrm{E}-21 \\
2.67 \mathrm{E}-20 \\
\dagger 7.25 \mathrm{E}-22 \\
1.04 \mathrm{E}-15\end{array}$ & $\begin{array}{l}35.7 \\
\dagger 900 . \\
\dagger 2096 \\
526 . \\
\dagger 1338\end{array}$ & $\begin{array}{l}5.82 \mathrm{E}-16 \\
7.50 \mathrm{E}-21 \\
\dagger 4.06 \mathrm{E}-21 \\
3.00 \mathrm{E}-20 \\
\dagger 1.50 \mathrm{E}-21 \\
5.82 \mathrm{E}-16\end{array}$ & $\begin{array}{l}49.3 \\
947 . \\
\dagger 2158 . \\
546 . \\
\dagger 1164 .\end{array}$ & $\begin{array}{l}6.27 \mathrm{E}-17 \\
2.32 \mathrm{E}-20 \\
3.02 \mathrm{E}-21 \\
4.69 \mathrm{E}-20 \\
1.06 \mathrm{E}-20 \\
6.28 \mathrm{E}-17\end{array}$ & $\begin{array}{l}104 . \\
1019 . \\
2264 . \\
619 . \\
1287 .\end{array}$ \\
\hline $\begin{array}{l}\text { DC } \\
\text { DC+SS } \\
\text { DC+DS } \\
\text { DC+SPEX } \\
\text { DC+DPEX } \\
\text { DC SUM }\end{array}$ & $\begin{array}{l}1.38 \mathrm{E}-17 \\
+9.86 \mathrm{E}-22 \\
+4.93 \mathrm{E}-22 \\
+4.93 \mathrm{E}-21 \\
+4.93 \mathrm{E}-22 \\
1.38 \mathrm{E}-17\end{array}$ & $\begin{array}{l}-21.4 \\
\dagger 808 \\
\dagger 1669 \\
\dagger 399 \\
\dagger 779\end{array}$ & $\begin{array}{l}4.38 \mathrm{E}-17 \\
\dagger 1.26 \mathrm{E}-20 \\
\dagger 4.19 \mathrm{E}-21 \\
3.74 \mathrm{E}-19 \\
\dagger 4.19 \mathrm{E}-21 \\
4.42 \mathrm{E}-17\end{array}$ & $\begin{array}{l}-11.6 \\
+828 . \\
\dagger 1799 . \\
457 . \\
+888\end{array}$ & $\begin{array}{l}5.48 \mathrm{E}-17 \\
3.28 \mathrm{E}-20 \\
\dagger 1.01 \mathrm{E}-20 \\
5.78 \mathrm{E}-19 \\
\dagger 1.01 \mathrm{E}-20 \\
5.54 \mathrm{E}-17\end{array}$ & $\begin{array}{l}32.0 \\
890 \\
\dagger 2047 \\
511 \\
\dagger 1032\end{array}$ & $\begin{array}{l}3.47 \mathrm{E}-17 \\
6.60 \mathrm{E}-20 \\
\dagger 1.93 \mathrm{E}-20 \\
8.84 \mathrm{E}-19 \\
1.32 \mathrm{E}-20 \\
3.57 \mathrm{E}-17\end{array}$ & $\begin{array}{l}78.8 \\
943 . \\
\dagger 2140 . \\
569 . \\
1381 .\end{array}$ & $\begin{array}{l}1.79 \mathrm{E}-17 \\
1.03 \mathrm{E}-19 \\
1.76 \mathrm{E}-20 \\
5.31 \mathrm{E}-19 \\
3.84 \mathrm{E}-20 \\
1.86 \mathrm{E}-17\end{array}$ & $\begin{array}{l}106 . \\
1003 . \\
2215 . \\
603 . \\
1221 .\end{array}$ & $\begin{array}{l}2.53 \mathrm{E}-18 \\
7.33 \mathrm{E}-20 \\
9.80 \mathrm{E}-21 \\
1.31 \mathrm{E}-19 \\
3.82 \mathrm{E}-20 \\
2.78 \mathrm{E}-18\end{array}$ & $\begin{array}{l}215 . \\
1130 . \\
2375 . \\
730 . \\
1398 .\end{array}$ \\
\hline $\begin{array}{l}\text { TEX } \\
\text { TEX+SS } \\
\text { TEX+DS } \\
\text { TEX+SPEX } \\
\text { TEX+DPEX } \\
\text { TEX SUM }\end{array}$ & $\begin{array}{l}1.04 \mathrm{E}-18 \\
+5.20 \mathrm{E}-25 \\
\dagger 1.04 \mathrm{E}-25 \\
\dagger 2.08 \mathrm{E}-21 \\
+5.20 \mathrm{E}-25 \\
1.04 \mathrm{E}-18\end{array}$ & $\begin{array}{l}7.70 \\
\dagger 837 \\
\dagger 1698 \\
\dagger 428 \\
\dagger 808\end{array}$ & $\begin{array}{l}1.17 \mathrm{E}-17 \\
\dagger 1.17 \mathrm{E}-23 \\
\dagger 5.85 \mathrm{E}-24 \\
3.16 \mathrm{E}-20 \\
5.85 \mathrm{E}-24 \\
1.17 \mathrm{E}-17\end{array}$ & $\begin{array}{l}7.70 \\
\dagger 847 . \\
\dagger 1818 . \\
477 . \\
908 .\end{array}$ & $\begin{array}{l}1.69 \mathrm{E}-16 \\
\dagger 7.90 \mathrm{E}-22 \\
\dagger 1.58 \mathrm{E}-22 \\
\dagger 1.58 \mathrm{E}-20 \\
\dagger 1.58 \mathrm{E}-22 \\
1.69 \mathrm{E}-16\end{array}$ & $\begin{array}{l}7.70 \\
+866 \\
+2018 \\
\dagger 487 \\
+1008\end{array}$ & $\begin{array}{l}2.92 \mathrm{E}-16 \\
+9.73 \mathrm{E}-22 \\
\dagger 4.87 \mathrm{E}-22 \\
\dagger 9.73 \mathrm{E}-22 \\
\dagger 4.87 \mathrm{E}-22 \\
2.92 \mathrm{E}-16\end{array}$ & $\begin{array}{l}7.70 \\
\dagger 872 . \\
\dagger 2068 . \\
\dagger 498 \\
\dagger 1310\end{array}$ & $\begin{array}{l}4.07 \mathrm{E}-16 \\
+8.48 \mathrm{E}-21 \\
+1.70 \mathrm{E}-21 \\
+8.48 \mathrm{E}-21 \\
+1.70 \mathrm{E}-21 \\
4.07 \mathrm{E}-16\end{array}$ & $\begin{array}{l}7.70 \\
\dagger 905 . \\
\dagger 2116 \\
\dagger 505 \\
\dagger 1123\end{array}$ & $\begin{array}{l}6.85 \mathrm{E}-16 \\
+2.28 \mathrm{E}-20 \\
+1.14 \mathrm{E}-20 \\
+2.28 \mathrm{E}-20 \\
+1.14 \mathrm{E}-20 \\
6.85 \mathrm{E}-16\end{array}$ & $\begin{array}{l}7.70 \\
\dagger 923 . \\
\dagger 2167 \\
\dagger 523 \\
\dagger 1191\end{array}$ \\
\hline Channel & $\begin{array}{l}\text { Energy } \\
500 \mathrm{keV} / \mathrm{u} \\
\text { cross } \\
\text { section } \\
\mathrm{cm}^{2}\end{array}$ & $\begin{array}{l}\text { Eloss } \\
\mathrm{eV}\end{array}$ & $\begin{array}{l}1000 \\
\text { cross } \\
\text { section } \\
\mathrm{cm}^{2}\end{array}$ & $\begin{array}{l}\text { Eloss } \\
\mathrm{eV}\end{array}$ & $\begin{array}{l}2000 \\
\text { cross } \\
\text { section } \\
\mathrm{cm}^{2}\end{array}$ & $\begin{array}{l}\text { Eloss } \\
\text { eV }\end{array}$ & $\begin{array}{l}5000 \\
\text { cross } \\
\text { section } \\
\mathrm{cm}^{2}\end{array}$ & $\begin{array}{l}\text { Eloss } \\
\mathrm{eV}\end{array}$ & $\begin{array}{l}10000 \\
\text { cross } \\
\text { section } \\
\mathrm{cm}^{2}\end{array}$ & $\begin{array}{l}\text { Eloss } \\
\mathrm{eV}\end{array}$ & $\begin{array}{l}25000 \\
\text { cross } \\
\text { section } \\
\mathrm{cm}^{2}\end{array}$ & $\begin{array}{l}\text { Eloss } \\
\mathrm{eV}\end{array}$ \\
\hline $\begin{array}{l}\text { SI } \\
\text { SI+SS } \\
\text { SI+DS } \\
\text { SI+SPEX } \\
\text { SI+DPEX } \\
\text { SI SUM }\end{array}$ & $\begin{array}{l}* 1.69 \mathrm{E}-15 \\
* 1.35 \mathrm{E}-18 \\
* 3.98 \mathrm{E}-19 \\
* 1.44 \mathrm{E}-18 \\
* 5.77 \mathrm{E}-19 \\
* 1.69 \mathrm{E}-15\end{array}$ & $\begin{array}{l}* 35.4 \\
* 991 . \\
* 2169 . \\
* 578 . \\
* 1246 .\end{array}$ & $\begin{array}{l}* 1.02 \mathrm{E}-15 \\
* 2.22 \mathrm{E}-18 \\
* 8.24 \mathrm{E}-19 \\
* 2.35 \mathrm{E}-18 \\
* 1.09 \mathrm{E}-18 \\
* 1.03 \mathrm{E}-15\end{array}$ & $\begin{array}{l}* 39.2 \\
* 1124 . \\
* 2357 \\
* 589 . \\
* 1269\end{array}$ & $\begin{array}{l}{ }^{*} 6.11 \mathrm{E}-16 \\
* 2.14 \mathrm{E}-18 \\
* 1.44 \mathrm{E}-18 \\
* 2.21 \mathrm{E}-18 \\
* 1.44 \mathrm{E}-18 \\
* 6.18 \mathrm{E}-16\end{array}$ & $\begin{array}{l}* 45.9 \\
* 1385 . \\
* 2940 . \\
* 592 . \\
* 1288 .\end{array}$ & $\begin{array}{l}* 2.94 \mathrm{E}-16 \\
* 1.83 \mathrm{E}-18 \\
* 1.11 \mathrm{E}-18 \\
* 1.97 \mathrm{E}-18 \\
* 1.11 \mathrm{E}-18 \\
* 3.00 \mathrm{E}-16\end{array}$ & $\begin{array}{l}* 55.6 \\
* 1836 . \\
* 3537 . \\
* 600 . \\
* 1270\end{array}$ & $\begin{array}{l}* 1.63 \mathrm{E}-16 \\
* 1.43 \mathrm{E}-18 \\
* 6.17 \mathrm{E}-19 \\
1.55 \mathrm{E}-18 \\
* 9.38 \mathrm{E}-19 \\
* 1.68 \mathrm{E}-16\end{array}$ & $\begin{array}{l}* 62.2 \\
* 2197 \\
* 4034 \\
* 604 \\
* 129 \\
* 12 .\end{array}$ & $\begin{array}{l}* 7.39 \mathrm{E}-17 \\
* 9.77 \mathrm{E}-19 \\
* 3.89 \mathrm{E}-19 \\
* 1.05 \mathrm{E}-18 \\
* 4.38 \mathrm{E}-19 \\
* 7.68 \mathrm{E}-17\end{array}$ & $\begin{array}{l}* 69.6 \\
* 2566 . \\
* 5468 . \\
* 610 . \\
* 1319 .\end{array}$ \\
\hline $\begin{array}{l}\text { DI } \\
\text { DI+SS } \\
\text { DI+DS } \\
\text { DI+SPEX } \\
\text { DI+DPEX } \\
\text { DI SUM }\end{array}$ & $\begin{array}{l}3.25 \mathrm{E}-16 \\
2.83 \mathrm{E}-18 \\
1.25 \mathrm{E}-18 \\
4.03 \mathrm{E}-18 \\
1.76 \mathrm{E}-18 \\
3.35 \mathrm{E}-16\end{array}$ & $\begin{array}{l}198 . \\
1153 . \\
2332 . \\
741 . \\
1409 .\end{array}$ & $\begin{array}{l}1.52 \mathrm{E}-16 \\
2.52 \mathrm{E}-18 \\
1.01 \mathrm{E}-18 \\
2.66 \mathrm{E}-18 \\
1.30 \mathrm{E}-18 \\
1.59 \mathrm{E}-16\end{array}$ & $\begin{array}{l}211 . \\
1295 . \\
2529 . \\
761 . \\
1441 .\end{array}$ & $\begin{array}{l}6.68 \mathrm{E}-17 \\
9.83 \mathrm{E}-19 \\
6.65 \mathrm{E}-19 \\
1.02 \mathrm{E}-18 \\
6.65 \mathrm{E}-19 \\
7.01 \mathrm{E}-17\end{array}$ & $\begin{array}{l}222 . \\
1561 . \\
3116 . \\
768 . \\
1464 .\end{array}$ & $\begin{array}{l}1.96 \mathrm{E}-17 \\
3.24 \mathrm{E}-19 \\
2.00 \mathrm{E}-19 \\
3.47 \mathrm{E}-19 \\
2.00 \mathrm{E}-19 \\
2.07 \mathrm{E}-17\end{array}$ & $\begin{array}{l}232 . \\
2012 . \\
3713 . \\
776 . \\
1446 .\end{array}$ & $\begin{array}{l}7.05 \mathrm{E}-18 \\
1.42 \mathrm{E}-19 \\
6.17 \mathrm{E}-20 \\
1.53 \mathrm{E}-19 \\
9.38 \mathrm{E}-20 \\
7.50 \mathrm{E}-18\end{array}$ & $\begin{array}{l}242 . \\
2377 . \\
4214 . \\
784 . \\
1479 .\end{array}$ & $\begin{array}{l}1.66 \mathrm{E}-18 \\
5.62 \mathrm{E}-20 \\
1.85 \mathrm{E}-20 \\
4.99 \mathrm{E}-20 \\
2.08 \mathrm{E}-20 \\
1.81 \mathrm{E}-18\end{array}$ & $\begin{array}{l}246 . \\
2642 . \\
5644 . \\
786 . \\
1495 .\end{array}$ \\
\hline $\begin{array}{l}\text { TI } \\
\text { TI+SS } \\
\text { TI+DS } \\
\text { TI+SPEX } \\
\text { TI+DPEX } \\
\text { TI SUM }\end{array}$ & $\begin{array}{l}6.48 \mathrm{E}-18 \\
2.57 \mathrm{E}-19 \\
8.28 \mathrm{E}-20 \\
2.70 \mathrm{E}-19 \\
1.17 \mathrm{E}-19 \\
7.21 \mathrm{E}-18\end{array}$ & $\begin{array}{l}305 . \\
1260 . \\
2439 . \\
848 . \\
1516 .\end{array}$ & $\begin{array}{l}3.36 \mathrm{E}-19 \\
1.93 \mathrm{E}-20 \\
7.90 \mathrm{E}-21 \\
2.04 \mathrm{E}-20 \\
9.95 \mathrm{E}-21 \\
3.94 \mathrm{E}-19\end{array}$ & $\begin{array}{l}576 . \\
1660 . \\
2894 . \\
1126 . \\
1806 .\end{array}$ & $\begin{array}{l}1.95 \mathrm{E}-20 \\
6.50 \mathrm{E}-22 \\
3.85 \mathrm{E}-22 \\
6.50 \mathrm{E}-22 \\
3.85 \mathrm{E}-22 \\
2.16 \mathrm{E}-20\end{array}$ & $\begin{array}{l}1124 . \\
2463 . \\
4018 . \\
1670 . \\
2366 .\end{array}$ & $\begin{array}{l}4.26 \mathrm{E}-22 \\
4.00 \mathrm{E}-24 \\
2.00 \mathrm{E}-24 \\
4.00 \mathrm{E}-24 \\
2.00 \mathrm{E}-24 \\
4.38 \mathrm{E}-22\end{array}$ & $\begin{array}{l}2752 . \\
4532 . \\
6233 . \\
3296 . \\
3966 .\end{array}$ & $\begin{array}{l}1.48 \mathrm{E}-23 \\
3.73 \mathrm{E}-26 \\
3.73 \mathrm{E}-26 \\
3.73 \mathrm{E}-26 \\
3.73 \mathrm{E}-26 \\
1.49 \mathrm{E}-23\end{array}$ & $\begin{array}{l}5451 . \\
7586 . \\
9423 . \\
5993 . \\
6688 .\end{array}$ & $\begin{array}{l}4.31 \mathrm{E}-25 \\
1.08 \mathrm{E}-28 \\
1.08 \mathrm{E}-28 \\
1.08 \mathrm{E}-28 \\
1.08 \mathrm{E}-28 \\
4.31 \mathrm{E}-25\end{array}$ & $\begin{array}{c}13544 . \\
15940 . \\
18942 . \\
14084 . \\
14793 .\end{array}$ \\
\hline
\end{tabular}


Table 9 (continued)

\begin{tabular}{|c|c|c|c|c|c|c|c|c|c|c|c|c|}
\hline DCAI & $3.20 \mathrm{E}-20$ & $\ddagger 547$. & $9.53 \mathrm{E}-23$ & $\ddagger 902$. & $8.00 \mathrm{E}-26$ & $\dagger 1700$ & $9.00 \mathrm{E}-30$ & +3000 & $8.91 \mathrm{E}-33$ & +4500 & $7.99 \mathrm{E}-37$ & $\dagger 7000$ \\
\hline $\mathrm{DCAI}+\mathrm{SS}$ & $2.25 \mathrm{E}-21$ & 1502. & $6.70 \mathrm{E}-24$ & 1986. & $6.77 \mathrm{E}-27$ & 3039. & $4.55 \mathrm{E}-31$ & 4780 & $4.09 \mathrm{E}-35$ & 6635. & $3.64 \mathrm{E}-40$ & 9396. \\
\hline $\mathrm{DCAI}+\mathrm{DS}$ & $7.58 \mathrm{E}-22$ & 2681. & $2.79 \mathrm{E}-24$ & 3220. & $3.33 \mathrm{E}-27$ & 4594. & $4.55 \mathrm{E}-31$ & 6481. & $4.09 \mathrm{E}-36$ & 8472. & $3.64 \mathrm{E}-41$ & 12398. \\
\hline DCAI+SPEX & $2.34 \mathrm{E}-21$ & 1090. & $7.26 \mathrm{E}-24$ & 1452. & $6.67 \mathrm{E}-27$ & 2246. & $4.55 \mathrm{E}-31$ & 3544. & $4.09 \mathrm{E}-35$ & 5042. & $3.64 \mathrm{E}-40$ & 7540. \\
\hline DCAI+DPEX & $1.07 \mathrm{E}-21$ & 1758. & $3.91 \mathrm{E}-24$ & 2132. & $3.33 \mathrm{E}-27$ & 2942. & $4.55 \mathrm{E}-31$ & 4214. & $4.09 \mathrm{E}-36$ & 5737. & $3.64 \mathrm{E}-41$ & 8249. \\
\hline DCAI SUM & $\ddagger 3.84 \mathrm{E}-20$ & & $\ddagger 1.16 \mathrm{E}-22$ & & $1.00 \mathrm{E}-25$ & & $1.08 \mathrm{E}-29$ & & $9.00 \mathrm{E}-33$ & & $8.00 \mathrm{E}-37$ & \\
\hline SC & $1.74 \mathrm{E}-18$ & 231. & $1.09 \mathrm{E}-19$ & 431. & $1.49 \mathrm{E}-20$ & 976. & $2.67 \mathrm{E}-22$ & 2610. & $1.70 \mathrm{E}-23$ & 5333. & $8.62 \mathrm{E}-25$ & 13502. \\
\hline $\mathrm{SC}+\mathrm{SS}$ & $1.70 \mathrm{E}-20$ & 1186. & $3.85 \mathrm{E}-21$ & 1515. & $4.75 \mathrm{E}-22$ & 2315. & $6.09 \mathrm{E}-24$ & 4390. & $6.06 \mathrm{E}-25$ & 7468. & $2.16 \mathrm{E}-30$ & 15898. \\
\hline $\mathrm{SC}+\mathrm{DS}$ & $4.35 \mathrm{E}-21$ & 2365. & $1.48 \mathrm{E}-21$ & 2749. & $2.83 \mathrm{E}-22$ & 3870. & $3.65 E-24$ & 6091. & $4.55 \mathrm{E}-25$ & 9305. & $2.16 \mathrm{E}-30$ & 18900. \\
\hline SC+SPEX & $1.80 \mathrm{E}-20$ & 774. & $4.10 \mathrm{E}-21$ & 981. & $5.01 \mathrm{E}-22$ & 1522. & $7.30 \mathrm{E}-24$ & 3154. & $6.06 \mathrm{E}-25$ & 5875. & $2.16 \mathrm{E}-30$ & 14042. \\
\hline SC+DPEX & $6.72 \mathrm{E}-21$ & 1442. & $1.90 \mathrm{E}-21$ & 1661. & $2.83 \mathrm{E}-22$ & 2218. & $3.65 \mathrm{E}-24$ & 3824. & $4.55 \mathrm{E}-25$ & 6570. & $2.16 \mathrm{E}-30$ & 14751. \\
\hline SC SUM & $1.79 \mathrm{E}-18$ & & $1.20 \mathrm{E}-19$ & & $1.64 \mathrm{E}-20$ & & $2.88 \mathrm{E}-22$ & & $1.91 \mathrm{E}-23$ & & $8.62 \mathrm{E}-25$ & \\
\hline DC & $2.40 \mathrm{E}-20$ & 480. & $3.09 \mathrm{E}-22$ & 904. & $\dagger 2.76 \mathrm{E}-24$ & $\dagger 1993$. & $\dagger 3.00 \mathrm{E}-27$ & $\dagger 5261$ & $\dagger 2.00 \mathrm{E}-29$ & $\dagger 10630$ & $\dagger 2.00 \mathrm{E}-32$ & $\dagger 26969$ \\
\hline $\mathrm{DC}+\mathrm{SS}$ & $2.37 \mathrm{E}-21$ & 1435. & $3.28 \mathrm{E}-23$ & 1988. & $2.30 \mathrm{E}-25$ & 3332. & $7.50 \mathrm{E}-31$ & 7041. & $5.00 \mathrm{E}-34$ & 12765. & $5.00 \mathrm{E}-38$ & 29365. \\
\hline $\mathrm{DC}+\mathrm{DS}$ & $8.40 \mathrm{E}-22$ & 2614. & $1.46 \mathrm{E}-23$ & 3222. & $1.15 \mathrm{E}-25$ & 4887. & $7.50 \mathrm{E}-31$ & 8742. & $5.00 \mathrm{E}-34$ & 14602. & $5.00 \mathrm{E}-38$ & 32367. \\
\hline $\mathrm{DC}+\mathrm{SPEX}$ & $2.46 \mathrm{E}-21$ & 1023. & $3.46 \mathrm{E}-23$ & 1454. & $2.30 \mathrm{E}-25$ & 2539. & $7.50 \mathrm{E}-31$ & 5805. & $5.00 \mathrm{E}-34$ & 11172. & $5.00 \mathrm{E}-38$ & 27509. \\
\hline $\mathrm{DC}+\mathrm{DPEX}$ & $1.17 \mathrm{E}-21$ & 1691. & $1.73 \mathrm{E}-23$ & 2134. & $1.15 \mathrm{E}-25$ & 3245. & $7.50 \mathrm{E}-31$ & 6475. & $5.00 \mathrm{E}-34$ & 11867. & $5.00 \mathrm{E}-38$ & 28218. \\
\hline DC SUM & $3.08 \mathrm{E}-20$ & & $4.08 \mathrm{E}-22$ & & $\ddagger 3.45 \mathrm{E}-24$ & & $3.00 \mathrm{E}-27$ & & $2.00 \mathrm{E}-29$ & & $2.00 \mathrm{E}-32$ & \\
\hline TEX & $8.96 \mathrm{E}-16$ & 7.70 & $7.65 \mathrm{E}-16$ & 7.70 & $5.18 \mathrm{E}-16$ & 7.70 & $2.76 \mathrm{E}-16$ & 7.70 & $1.49 \mathrm{E}-16$ & 7.70 & $6.09 \mathrm{E}-17$ & 7.70 \\
\hline TEX+SS & $7.43 \mathrm{E}-20$ & 963. & $4.93 \mathrm{E}-19$ & 1092. & $9.65 E-19$ & 1347. & $8.93 \mathrm{E}-19$ & 1788. & $6.89 \mathrm{E}-19$ & 2142. & $3.77 \mathrm{E}-19$ & 2404. \\
\hline TEX+DS & $4.09 \mathrm{E}-20$ & 2141. & $3.10 \mathrm{E}-19$ & 2325. & $7.91 \mathrm{E}-19$ & 2901. & $6.95 \mathrm{E}-19$ & 3489. & $4.50 \mathrm{E}-19$ & 3978. & $2.68 \mathrm{E}-19$ & 5406. \\
\hline TEX+SPEX & $7.43 \mathrm{E}-20$ & 551. & $5.08 \mathrm{E}-19$ & 558. & $9.82 \mathrm{E}-19$ & 554. & $9.27 \mathrm{E}-19$ & 552. & $7.15 \mathrm{E}-19$ & 550. & $4.44 \mathrm{E}-19$ & 548. \\
\hline TEX+DPEX & $4.83 \mathrm{E}-20$ & 1219. & $3.51 \mathrm{E}-19$ & 1238. & $7.91 \mathrm{E}-19$ & 1250. & $6.95 \mathrm{E}-19$ & 1222. & $5.56 \mathrm{E}-19$ & 1245. & $2.85 \mathrm{E}-19$ & 1257. \\
\hline TEX SUM & $8.96 \mathrm{E}-16$ & & $7.67 \mathrm{E}-16$ & & $5.22 \mathrm{E}-16$ & & $2.79 \mathrm{E}-16$ & & $1.51 \mathrm{E}-16$ & & $6.23 \mathrm{E}-17$ & \\
\hline
\end{tabular}

$\doteqdot$ Extrapolated. $\quad \ddagger$ Significantly improved. $\quad{ }^{*}$ Includes Born correction. 
Table 10

The integral cross section and associated average energy loss for NSIM and SIM processes in $\mathrm{O}^{7+}+\mathrm{H}_{2}$ collisions with impact energies between 1 and $25000 \mathrm{keV} / \mathrm{u}$.

\begin{tabular}{|c|c|c|c|c|c|c|c|c|c|c|c|c|}
\hline \multirow[t]{2}{*}{ Channel } & \multicolumn{11}{|l|}{ Energy } & \\
\hline & $\begin{array}{l}\text { cross } \\
\text { section } \\
\mathrm{cm}^{2}\end{array}$ & $\begin{array}{l}\text { Eloss } \\
\mathrm{eV}\end{array}$ & $\begin{array}{l}\text { cross } \\
\text { section } \\
\mathrm{cm}^{2}\end{array}$ & $\begin{array}{l}\text { Eloss } \\
\mathrm{eV}\end{array}$ & $\begin{array}{l}\text { cross } \\
\text { section } \\
\mathrm{cm}^{2}\end{array}$ & $\begin{array}{l}\text { Eloss } \\
\mathrm{eV}\end{array}$ & $\begin{array}{l}\text { cross } \\
\text { section } \\
\mathrm{cm}^{2}\end{array}$ & $\begin{array}{l}\text { Eloss } \\
\mathrm{eV}\end{array}$ & $\begin{array}{l}\text { cross } \\
\text { section } \\
\mathrm{cm}^{2}\end{array}$ & $\begin{array}{l}\text { Eloss } \\
\mathrm{eV}\end{array}$ & $\begin{array}{l}\text { cross } \\
\text { section } \\
\mathrm{cm}^{2}\end{array}$ & $\begin{array}{l}\text { Eloss } \\
\mathrm{eV}\end{array}$ \\
\hline SI & $1.09 \mathrm{E}-19$ & $\ddagger 16.6$ & $1.99 \mathrm{E}-18$ & 23.8 & $* 4.89 \mathrm{E}-16$ & $* 19.4$ & $1.04 \mathrm{E}-15$ & $* 26.2$ & $* 1.80 \mathrm{E}-15$ & $* 32.2$ & $* 3.28 \mathrm{E}-15$ & $* 34.2$ \\
\hline $\mathrm{SI}+\mathrm{SS}$ & $\dagger 2.29 \mathrm{E}-24$ & $\dagger 988$. & $\dagger 6.08 \mathrm{E}-23$ & $\dagger 1015$ & $* 1.18 \mathrm{E}-20$ & * 1031 & $* 2.40 \mathrm{E}-20$ & * 1046 & $* 1.27 \mathrm{E}-19$ & * 1078 & $* 8.04 \mathrm{E}-19$ & * 1115 \\
\hline SI+SPEX & $\dagger 1.10 \mathrm{E}-21$ & +603. & $3.70 \mathrm{E}-20$ & 532. & $* 9.45 \mathrm{E}-20$ & $* 545$ & $* 2.10 \mathrm{E}-19$ & * 559 & $* 8.23 \mathrm{E}-19$ & * 570 & $* 2.66 \mathrm{E}-18$ & * 600 \\
\hline SI SUM & $\ddagger 1.10 \mathrm{E}-19$ & & $2.03 \mathrm{E}-18$ & & $* 4.89 \mathrm{E}-16$ & & $* 1.04 \mathrm{E}-15$ & & $* 1.80 \mathrm{E}-15$ & & $* 3.28 \mathrm{E}-15$ & \\
\hline DI & $\dagger 1.00 \mathrm{E}-24$ & +37.7 & $4.22 \mathrm{E}-21$ & 39.8 & $1.17 \mathrm{E}-18$ & 58.2 & $2.21 \mathrm{E}-17$ & 82.9 & $1.13 \mathrm{E}-16$ & 112. & $4.44 \mathrm{E}-16$ & 167. \\
\hline $\mathrm{DI}+\mathrm{SS}$ & $\dagger 5.00 \mathrm{E}-32$ & $\dagger 1009$ & $\dagger 4.22 \mathrm{E}-28$ & $\dagger 1031$ & $\dagger 1.00 \mathrm{E}-24$ & $\dagger 1070$ & $3.70 \mathrm{E}-21$ & 1102. & $5.11 \mathrm{E}-20$ & 1157. & $9.10 \mathrm{E}-19$ & 1247. \\
\hline DI+SPEX & $\dagger 5.00 \mathrm{E}-32$ & +624. & $\dagger 1.00 \mathrm{E}-25$ & $\dagger 548$. & $1.91 \mathrm{E}-21$ & 584. & $2.89 \mathrm{E}-20$ & 616. & $3.19 \mathrm{E}-19$ & 650. & $2.98 \mathrm{E}-18$ & 733. \\
\hline DI SUM & $\dagger 1.00 \mathrm{E}-24$ & & $4.22 \mathrm{E}-21$ & & $1.17 \mathrm{E}-18$ & & $2.21 \mathrm{E}-17$ & & $1.13 \mathrm{E}-16$ & & $4.48 \mathrm{E}-16$ & \\
\hline $\mathrm{TI}$ & $\dagger 1.98 \mathrm{E}-20$ & $\dagger-11.2$ & $4.50 \mathrm{E}-18$ & 3.05 & $8.99 \mathrm{E}-17$ & 46.5 & $3.12 \mathrm{E}-16$ & 59.3 & $4.90 \mathrm{E}-16$ & 89.4 & $2.18 \mathrm{E}-16$ & 186. \\
\hline $\mathrm{TI}+\mathrm{SS}$ & $+9.95 \mathrm{E}-25$ & +960 & $+4.53 \mathrm{E}-22$ & $\dagger 994$. & $1.46 \mathrm{E}-20$ & 1058. & $6.97 \mathrm{E}-20$ & 1079. & $3.56 \mathrm{E}-19$ & 1135. & $1.03 \mathrm{E}-18$ & 1266. \\
\hline TI+SPEX & $\dagger 1.99 \mathrm{E}-22$ & +575. & $6.41 \mathrm{E}-20$ & 511. & $1.59 \mathrm{E}-19$ & 573. & $6.31 \mathrm{E}-19$ & 592. & $2.32 \mathrm{E}-18$ & 627. & $3.29 \mathrm{E}-18$ & 752. \\
\hline TI SUM & $+2.00 \mathrm{E}-20$ & & $4.56 \mathrm{E}-18$ & & $9.01 \mathrm{E}-17$ & & $3.13 \mathrm{E}-16$ & & $4.93 \mathrm{E}-16$ & & $2.22 \mathrm{E}-16$ & \\
\hline DCAI & $2.85 \mathrm{E}-15$ & 611. & $2.29 \mathrm{E}-15$ & 689. & $1.52 \mathrm{E}-15$ & 860. & $1.01 \mathrm{E}-15$ & 943. & $5.12 \mathrm{E}-16$ & 1007. & $2.89 \mathrm{E}-17$ & 1202. \\
\hline $\mathrm{DCAI}+\mathrm{SS}$ & $\dagger 1.37 \mathrm{E}-19$ & $\dagger 1582$ & $\dagger 2.15 \mathrm{E}-19$ & $\dagger 1680$ & $2.45 \mathrm{E}-19$ & 1871. & $3.24 \mathrm{E}-19$ & 1962. & $5.11 \mathrm{E}-19$ & 2052. & $2.02 \mathrm{E}-19$ & 2282. \\
\hline DCAI+SPEX & $3.19 \mathrm{E}-18$ & 1197. & $3.47 \mathrm{E}-18$ & 1197. & $3.00 \mathrm{E}-18$ & 1386. & $2.79 \mathrm{E}-18$ & 1476. & $3.24 \mathrm{E}-18$ & 1545. & $6.79 \mathrm{E}-19$ & 1768. \\
\hline DCAI SUM & $2.85 \mathrm{E}-15$ & & $2.29 \mathrm{E}-15$ & & $1.52 \mathrm{E}-15$ & & $1.01 \mathrm{E}-15$ & & $5.16 \mathrm{E}-16$ & & $2.98 \mathrm{E}-17$ & \\
\hline SC & $2.61 \mathrm{E}-15$ & -26.6 & $2.22 \mathrm{E}-15$ & -21.7 & $1.74 \mathrm{E}-15$ & 15.4 & $1.28 \mathrm{E}-15$ & 29.0 & $7.59 \mathrm{E}-16$ & 42.6 & $8.44 \mathrm{E}-17$ & 97.1 \\
\hline $\mathrm{SC}+\mathrm{SS}$ & $\dagger 2.60 \mathrm{E}-22$ & $\dagger 945$ & $\dagger 4.42 \mathrm{E}-22$ & $\dagger 970$ & $\dagger 8.56 \mathrm{E}-22$ & $\dagger 1027$ & $\dagger 2.40 \mathrm{E}-21$ & $\dagger 1048$ & $4.96 \mathrm{E}-21$ & 1088. & $8.49 \mathrm{E}-21$ & 1178. \\
\hline SC+SPEX & $\dagger 7.80 \mathrm{E}-20$ & $\dagger 559$. & $9.57 \mathrm{E}-20$ & 486. & $\dagger 5.13 \mathrm{E}-20$ & $\dagger 541$. & $+3.60 \mathrm{E}-20$ & +562. & $1.73 \mathrm{E}-20$ & 581. & $3.52 \mathrm{E}-20$ & 663. \\
\hline SC SUM & $2.61 \mathrm{E}-15$ & & $2.22 \mathrm{E}-15$ & & $1.74 \mathrm{E}-15$ & & $1.28 \mathrm{E}-15$ & & $7.59 \mathrm{E}-16$ & & $8.44 \mathrm{E}-17$ & \\
\hline DC & $6.94 \mathrm{E}-18$ & -40.6 & $6.44 \mathrm{E}-18$ & -30.8 & $1.29 \mathrm{E}-17$ & 39.2 & $1.47 \mathrm{E}-17$ & 66.5 & $1.03 \mathrm{E}-17$ & 93.7 & $1.74 \mathrm{E}-18$ & 203. \\
\hline $\mathrm{DC}+\mathrm{SS}$ & $\dagger 3.34 \mathrm{E}-21$ & † 931 & $\dagger 6.17 \mathrm{E}-21$ & $\dagger 961$. & $2.03 \mathrm{E}-20$ & 1051. & $3.26 \mathrm{E}-20$ & 1086. & $4.16 \mathrm{E}-20$ & 1139. & $3.39 \mathrm{E}-20$ & 1283. \\
\hline $\mathrm{DC}+\mathrm{SPEX}$ & $+6.68 \mathrm{E}-20$ & $\dagger 545$. & $1.00 \mathrm{E}-19$ & 477. & $2.13 \mathrm{E}-19$ & 565. & $2.17 \mathrm{E}-19$ & 600. & $2.96 \mathrm{E}-19$ & 632. & $1.11 \mathrm{E}-19$ & 769. \\
\hline DC SUM & $7.01 \mathrm{E}-18$ & & $6.55 \mathrm{E}-18$ & & $1.31 \mathrm{E}-17$ & & $1.49 \mathrm{E}-17$ & & $1.06 \mathrm{E}-17$ & & $1.88 \mathrm{E}-18$ & \\
\hline TEX & $1.25 \mathrm{E}-18$ & 7.70 & $7.88 \mathrm{E}-18$ & 7.70 & $1.59 \mathrm{E}-16$ & 7.70 & $2.96 \mathrm{E}-16$ & 7.70 & $4.09 \mathrm{E}-16$ & 7.70 & $7.45 \mathrm{E}-16$ & 7.70 \\
\hline TEX+SS & $\dagger 1.24 \mathrm{E}-22$ & † 979. & $\dagger 3.85 \mathrm{E}-22$ & † 999. & $\dagger 1.57 \mathrm{E}-21$ & $\dagger 1019$ & $\dagger 1.47 \mathrm{E}-21$ & $\dagger 1027$ & $+4.01 \mathrm{E}-21$ & $\dagger 1053$ & $\dagger 3.19 \mathrm{E}-20$ & $\dagger 1088$ \\
\hline TEX+SPEX & $9.02 \mathrm{E}-21$ & 594. & $1.51 \mathrm{E}-20$ & 516. & $\dagger 1.57 \mathrm{E}-19$ & $\dagger 534$. & $\dagger 2.06 \mathrm{E}-19$ & $\dagger 541$. & $\dagger 2.00 \mathrm{E}-19$ & $\dagger 546$. & $\dagger 1.92 \mathrm{E}-19$ & $\dagger 574$ \\
\hline TEX SUM & $1.26 \mathrm{E}-18$ & & $7.90 \mathrm{E}-18$ & & $1.59 \mathrm{E}-16$ & & $2.96 \mathrm{E}-16$ & & $4.09 \mathrm{E}-16$ & & $7.45 \mathrm{E}-16$ & \\
\hline \multirow[t]{3}{*}{ Channel } & Energy & & & & & & & & & & & \\
\hline & $500 \mathrm{keV} / \mathrm{u}$ & & 1000 & & 2000 & & 5000 & & 10000 & & 25000 & \\
\hline & $\begin{array}{l}\text { cross } \\
\text { section } \\
\mathrm{cm}^{2}\end{array}$ & $\begin{array}{l}\text { Eloss } \\
\mathrm{eV}\end{array}$ & $\begin{array}{l}\text { cross } \\
\text { section } \\
\mathrm{cm}^{2}\end{array}$ & $\begin{array}{l}\text { Eloss } \\
\mathrm{eV}\end{array}$ & $\begin{array}{l}\text { cross } \\
\text { section } \\
\mathrm{cm}^{2}\end{array}$ & $\begin{array}{l}\text { Eloss } \\
\mathrm{eV}\end{array}$ & $\begin{array}{l}\text { cross } \\
\text { section } \\
\mathrm{cm}^{2}\end{array}$ & $\begin{array}{l}\text { Eloss } \\
\mathrm{eV}\end{array}$ & $\begin{array}{l}\text { cross } \\
\text { section } \\
\mathrm{cm}^{2}\end{array}$ & $\begin{array}{l}\text { Eloss } \\
\mathrm{eV}\end{array}$ & $\begin{array}{l}\text { cross } \\
\text { section } \\
\mathrm{cm}^{2}\end{array}$ & $\begin{array}{l}\text { Eloss } \\
\mathrm{eV}\end{array}$ \\
\hline SI & $* 2.13 \mathrm{E}-15$ & $* 33.7$ & ${ }^{*} 1.35 \mathrm{E}-15$ & $* 35.6$ & $* 8.12 \mathrm{E}-16$ & $* 40.0$ & $* 3.92 \mathrm{E}-16$ & $* 47.3$ & $* 2.18 \mathrm{E}-16$ & $* 49.2$ & $* 9.79 \mathrm{E}-17$ & $* 58.6$ \\
\hline $\mathrm{SI}+\mathrm{SS}$ & $* 1.43 \mathrm{E}-18$ & * 1137 & $* 2.15 \mathrm{E}-18$ & 1263. & $* 3.17 \mathrm{E}-18$ & * 1530 & $* 2.81 \mathrm{E}-18$ & * 2066 & $* 2.43 \mathrm{E}-18$ & * 2427 & $* 1.51 \mathrm{E}-18$ & * 2757 \\
\hline SI+SPEX & $* 1.72 \mathrm{E}-18$ & * 635 & $* 2.15 \mathrm{E}-18$ & $* 656$ & $* 3.54 \mathrm{E}-18$ & * 657 & $* 3.15 \mathrm{E}-18$ & * 655 & $* 2.34 \mathrm{E}-18$ & * 655 & $* 1.63 \mathrm{E}-18$ & * 662 \\
\hline SI SUM & $* 2.13 \mathrm{E}-15$ & & $* 1.35 \mathrm{E}-15$ & & $* 8.19 \mathrm{E}-16$ & & $* 3.98 \mathrm{E}-16$ & & $* 2.23 \mathrm{E}-16$ & & $* 1.01 \mathrm{E}-16$ & \\
\hline DI & $3.83 \mathrm{E}-16$ & 193. & $2.11 \mathrm{E}-16$ & 198. & $9.77 \mathrm{E}-17$ & 202. & $3.00 \mathrm{E}-17$ & 203. & $1.10 \mathrm{E}-17$ & 205. & $2.56 \mathrm{E}-18$ & 205. \\
\hline $\mathrm{DI}+\mathrm{SS}$ & $4.04 \mathrm{E}-18$ & 1296. & $3.81 \mathrm{E}-18$ & 1425. & $2.03 \mathrm{E}-18$ & 1692. & $6.47 \mathrm{E}-19$ & 2221. & $2.96 \mathrm{E}-19$ & 2582. & $8.29 \mathrm{E}-20$ & 2903. \\
\hline DI+SPEX & $4.80 \mathrm{E}-18$ & 794. & $3.81 \mathrm{E}-18$ & 818. & $2.25 \mathrm{E}-18$ & 819. & $7.23 \mathrm{E}-19$ & 811. & $2.85 \mathrm{E}-19$ & 811. & $8.96 \mathrm{E}-20$ & 808. \\
\hline DI SUM & $3.92 \mathrm{E}-16$ & & $2.19 \mathrm{E}-16$ & & $1.02 \mathrm{E}-16$ & & $3.14 \mathrm{E}-17$ & & $1.16 \mathrm{E}-17$ & & $2.73 \mathrm{E}-18$ & \\
\hline $\mathrm{TI}$ & $1.01 \mathrm{E}-17$ & $\S 360$. & $5.67 \mathrm{E}-19$ & 540. & $3.09 \mathrm{E}-20$ & 1077. & $7.35 \mathrm{E}-22$ & 2680. & $2.68 \mathrm{E}-23$ & +4836 & $5.99 \mathrm{E}-25$ & $\dagger 12980$. \\
\hline $\mathrm{TI}+\mathrm{SS}$ & $3.90 \mathrm{E}-19$ & 1463. & $3.89 \mathrm{E}-20$ & 1767. & $1.51 \mathrm{E}-21$ & 2567. & $1.41 \mathrm{E}-23$ & 4698. & $1.36 \mathrm{E}-25$ & 7213. & $5.45 \mathrm{E}-29$ & 15678. \\
\hline TI+SPEX & $4.64 \mathrm{E}-19$ & 961. & $3.89 \mathrm{E}-20$ & 1160. & $1.69 \mathrm{E}-21$ & 1694. & $2.26 \mathrm{E}-23$ & 3288. & $1.36 \mathrm{E}-25$ & 5442. & $5.45 \mathrm{E}-28$ & 13583. \\
\hline TI SUM & $1.10 \mathrm{E}-17$ & & $6.45 E-19$ & & $3.41 \mathrm{E}-20$ & & $7.72 \mathrm{E}-22$ & & $2.71 \mathrm{E}-23$ & & $6.00 \mathrm{E}-25$ & \\
\hline DCAI & $8.21 \mathrm{E}-20$ & $\ddagger 1634$. & $2.73 \mathrm{E}-22$ & $\ddagger 2204$. & $\dagger 3.75 E-25$ & $\dagger 2800$ & $+6.75 \mathrm{E}-29$ & +3600 & $\dagger 1.26 \mathrm{E}-31$ & $\dagger 4500$ & $\dagger 2.97 \mathrm{E}-35$ & $\dagger 6000$ \\
\hline $\mathrm{DCAI}+\mathrm{SS}$ & $5.00 \mathrm{E}-21$ & 2737. & $4.50 \mathrm{E}-23$ & 3431. & $6.25 \mathrm{E}-26$ & 4290. & $6.43 \mathrm{E}-30$ & 5618. & $1.27 \mathrm{E}-33$ & 6877. & $2.73 \mathrm{E}-38$ & 8698. \\
\hline DCAI+SPEX & $5.22 \mathrm{E}-21$ & 2235. & $4.50 \mathrm{E}-23$ & 2824. & $6.25 \mathrm{E}-26$ & 3417. & $1.61 \mathrm{E}-29$ & 4208. & $1.27 \mathrm{E}-32$ & 5106. & $2.73 E-37$ & 6603. \\
\hline DCAI SUM & $\ddagger 9.23 \mathrm{E}-20$ & & $\ddagger 3.63 \mathrm{E}-22$ & & $5.00 \mathrm{E}-25$ & & $9.00 \mathrm{E}-29$ & & $1.40 \mathrm{E}-31$ & & $3.00 \mathrm{E}-35$ & \\
\hline SC & $2.21 \mathrm{E}-18$ & 212. & $1.36 \mathrm{E}-19$ & 484. & $1.49 \mathrm{E}-20$ & 1029. & $2.66 \mathrm{E}-22$ & 2568. & $1.86 \mathrm{E}-23$ & 4781. & $4.00 \mathrm{E}-25$ & 12950. \\
\hline $\mathrm{SC}+\mathrm{SS}$ & $1.21 \mathrm{E}-20$ & 1315. & $5.13 \mathrm{E}-21$ & 1711. & $7.08 \mathrm{E}-22$ & 2519. & $1.08 \mathrm{E}-23$ & 4586. & $1.06 \mathrm{E}-24$ & 7158. & $2.00 \mathrm{E}-30$ & 15648. \\
\hline SC+SPEX & $1.50 \mathrm{E}-20$ & 813. & $5.13 \mathrm{E}-21$ & 1104. & $8.15 \mathrm{E}-22$ & 1646. & $1.15 \mathrm{E}-23$ & 3176. & $1.06 \mathrm{E}-24$ & 5387. & $2.00 \mathrm{E}-30$ & 13553. \\
\hline SC SUM & $2.24 \mathrm{E}-18$ & & $1.46 \mathrm{E}-19$ & & $1.64 \mathrm{E}-20$ & & $2.88 \mathrm{E}-22$ & & $2.07 \mathrm{E}-23$ & & $4.00 \mathrm{E}-25$ & \\
\hline DC & $2.31 \mathrm{E}-20$ & 446. & $3.74 \mathrm{E}-22$ & 990. & $4.00 \mathrm{E}-24$ & 2080. & $6.99 \mathrm{E}-27$ & 5347. & $9.00 \mathrm{E}-29$ & 10630. & $1.50 \mathrm{E}-31$ & 26969. \\
\hline $\mathrm{DC}+\mathrm{SS}$ & $2.18 \mathrm{E}-21$ & 1549. & $4.17 \mathrm{E}-23$ & 2217. & $5.00 \mathrm{E}-25$ & 3570. & $3.50 \mathrm{E}-30$ & 7365. & $4.50 \mathrm{E}-33$ & 13007. & $7.50 \mathrm{E}-37$ & 29667. \\
\hline $\mathrm{DC}+\mathrm{SPEX}$ & $2.72 \mathrm{E}-21$ & 1047. & $4.17 \mathrm{E}-23$ & 1610. & $5.00 \mathrm{E}-25$ & 2697. & $3.50 \mathrm{E}-30$ & 5955. & $4.50 \mathrm{E}-33$ & 11236. & $7.50 \mathrm{E}-37$ & 27572 . \\
\hline DC SUM & $2.80 \mathrm{E}-20$ & & $4.57 \mathrm{E}-22$ & & $5.00 \mathrm{E}-24$ & & $7.00 \mathrm{E}-27$ & & $9.00 \mathrm{E}-29$ & & $1.50 \mathrm{E}-31$ & \\
\hline TEX & $1.03 \mathrm{E}-15$ & 7.70 & $9.34 \mathrm{E}-16$ & 7.70 & $6.79 \mathrm{E}-16$ & 7.70 & $3.58 \mathrm{E}-16$ & 7.70 & $1.98 \mathrm{E}-16$ & 7.70 & $8.22 \mathrm{E}-17$ & 7.70 \\
\hline TEX+SS & $5.60 \mathrm{E}-20$ & 1111. & $3.85 \mathrm{E}-19$ & 1235. & $1.47 \mathrm{E}-18$ & 1498. & $1.73 \mathrm{E}-18$ & 2026. & $1.53 \mathrm{E}-18$ & 2385 . & $1.02 \mathrm{E}-18$ & 2706. \\
\hline TEX+SPEX & $5.60 \mathrm{E}-20$ & 609. & $3.85 \mathrm{E}-19$ & 628. & $1.55 \mathrm{E}-18$ & 625. & $1.83 \mathrm{E}-18$ & 616. & $1.50 \mathrm{E}-18$ & 614. & $1.07 \mathrm{E}-18$ & 611. \\
\hline TEX SUM & $1.03 \mathrm{E}-15$ & & $9.35 \mathrm{E}-16$ & & $6.82 \mathrm{E}-16$ & & $3.62 \mathrm{E}-16$ & & $2.01 \mathrm{E}-16$ & & $8.43 \mathrm{E}-17$ & \\
\hline
\end{tabular}

$\dagger$ Extrapolated. $\quad \ddagger$ Significantly improved. $\quad \S$ Corrects typographical error in Ref. [1]. $\quad{ }^{*}$ Includes Born correction. 
Table 11

The integral cross section and associated average energy loss for NSIM and SIM processes in $\mathrm{O}^{8+}+\mathrm{H}_{2}$ collisions with impact energies between 1 and 25000 keV/u.

\begin{tabular}{|c|c|c|c|c|c|c|c|c|c|c|c|c|}
\hline \multirow[t]{2}{*}{ Channel } & \multicolumn{12}{|l|}{ Energy } \\
\hline & $\begin{array}{l}\text { cross } \\
\text { section } \\
\mathrm{cm}^{2}\end{array}$ & $\begin{array}{l}\text { Eloss } \\
\mathrm{eV}\end{array}$ & $\begin{array}{l}\text { cross } \\
\text { section } \\
\mathrm{cm}^{2}\end{array}$ & $\begin{array}{l}\text { Eloss } \\
\text { eV }\end{array}$ & $\begin{array}{l}\text { cross } \\
\text { section } \\
\mathrm{cm}^{2}\end{array}$ & $\begin{array}{l}\text { Eloss } \\
\mathrm{eV}\end{array}$ & $\begin{array}{l}\text { cross } \\
\text { section } \\
\mathrm{cm}^{2}\end{array}$ & $\begin{array}{l}\text { Eloss } \\
\text { eV }\end{array}$ & $\begin{array}{l}\text { cross } \\
\text { section } \\
\mathrm{cm}^{2}\end{array}$ & $\begin{array}{l}\text { Eloss } \\
\mathrm{eV}\end{array}$ & $\begin{array}{l}\text { cross } \\
\text { section } \\
\mathrm{cm}^{2}\end{array}$ & $\begin{array}{l}\text { Eloss } \\
\text { eV }\end{array}$ \\
\hline SI & $\dagger 1.00 \mathrm{E}-22$ & $\dagger 15.4$ & $1.58 \mathrm{E}-18$ & 24.1 & $* 2.27 \mathrm{E}-16$ & $* 22.8$ & $* 1.17 \mathrm{E}-15$ & $* 24.9$ & $* 2.10 \mathrm{E}-15$ & $* 30.9$ & $* 3.06 \mathrm{E}-15$ & $* 38.9$ \\
\hline DI & $\dagger 1.00 E-33$ & $\dagger 35.7$ & $\dagger 1.00 \mathrm{E}-24$ & $\dagger 35.7$ & $7.60 \mathrm{E}-19$ & 53.6 & $1.60 \mathrm{E}-17$ & 77.8 & $9.57 \mathrm{E}-17$ & 109. & $5.32 \mathrm{E}-16$ & 170. \\
\hline TI & $\ddagger 3.24 \mathrm{E}-20$ & $\ddagger-22.3$ & $3.41 \mathrm{E}-18$ & -8.87 & $7.62 \mathrm{E}-17$ & 49.0 & $3.01 \mathrm{E}-16$ & 75.7 & $5.41 \mathrm{E}-16$ & 121. & $\S 2.88 \mathrm{E}-16$ & 176. \\
\hline DCAI & $3.39 E-15$ & 723. & $2.75 \mathrm{E}-15$ & 805. & $1.87 \mathrm{E}-15$ & 988. & $1.33 \mathrm{E}-15$ & 1076. & $7.44 \mathrm{E}-16$ & 1146. & $4.91 \mathrm{E}-17$ & 1343. \\
\hline SC & $2.90 \mathrm{E}-15$ & -18.9 & $2.45 \mathrm{E}-15$ & -14.0 & $2.00 \mathrm{E}-15$ & 18.5 & $1.53 \mathrm{E}-15$ & 32.1 & $9.55 E-16$ & 45.7 & $1.08 \mathrm{E}-16$ & 89.5 \\
\hline DC & $1.51 \mathrm{E}-17$ & -28.3 & $1.48 \mathrm{E}-17$ & -18.5 & $1.45 \mathrm{E}-17$ & 43.8 & $1.40 \mathrm{E}-17$ & 71.0 & $1.23 \mathrm{E}-17$ & 98.2 & $1.24 \mathrm{E}-18$ & 188. \\
\hline TEX & $8.29 \mathrm{E}-19$ & 7.70 & $5.48 \mathrm{E}-18$ & 7.70 & $1.51 \mathrm{E}-16$ & 7.70 & $2.88 \mathrm{E}-16$ & 7.70 & $4.07 \mathrm{E}-16$ & 7.70 & $7.76 \mathrm{E}-16$ & 7.70 \\
\hline \multirow[t]{3}{*}{ Channel } & \multicolumn{12}{|l|}{ Energy } \\
\hline & $500 \mathrm{keV} / \mathrm{u}$ & & 1000 & & 2000 & & 5000 & & 10000 & & 25000 & \\
\hline & $\begin{array}{l}\text { cross } \\
\text { section } \\
\mathrm{cm}^{2}\end{array}$ & $\begin{array}{l}\text { Eloss } \\
\mathrm{eV}\end{array}$ & $\begin{array}{l}\text { cross } \\
\text { section } \\
\mathrm{cm}^{2}\end{array}$ & $\begin{array}{l}\text { Eloss } \\
\mathrm{eV}\end{array}$ & $\begin{array}{l}\text { cross } \\
\text { section } \\
\mathrm{cm}^{2}\end{array}$ & $\begin{array}{l}\text { Eloss } \\
\mathrm{eV}\end{array}$ & $\begin{array}{l}\text { cross } \\
\text { section } \\
\mathrm{cm}^{2}\end{array}$ & $\begin{array}{l}\text { Eloss } \\
\text { eV }\end{array}$ & $\begin{array}{l}\text { cross } \\
\text { section } \\
\mathrm{cm}^{2}\end{array}$ & $\begin{array}{l}\text { Eloss } \\
\mathrm{eV}\end{array}$ & $\begin{array}{l}\text { cross } \\
\text { section } \\
\mathrm{cm}^{2}\end{array}$ & $\begin{array}{l}\text { Eloss } \\
\text { eV }\end{array}$ \\
\hline SI & $* 2.67 \mathrm{E}-15$ & $* 33.2$ & $* 1.70 \mathrm{E}-15$ & $* 33.7$ & $* 1.04 \mathrm{E}-15$ & $* 37.3$ & $* 5.07 \mathrm{E}-16$ & $* 43.0$ & $* 2.86 \mathrm{E}-16$ & $* 47.2$ & $* 1.30 \mathrm{E}-16$ & $* 51.8$ \\
\hline DI & $5.05 \mathrm{E}-16$ & 196. & $2.94 \mathrm{E}-16$ & 198. & $1.42 \mathrm{E}-16$ & 197. & $4.53 \mathrm{E}-17$ & 195. & $1.72 \mathrm{E}-17$ & 192. & $4.19 \mathrm{E}-18$ & 189. \\
\hline TI & $1.60 \mathrm{E}-17$ & 345. & $9.84 \mathrm{E}-19$ & 610. & $5.59 \mathrm{E}-20$ & 1125. & $1.01 \mathrm{E}-21$ & 2632. & $8.44 \mathrm{E}-23$ & 4664. & $\dagger 2.20 \mathrm{E}-24$ & $\dagger 12790$. \\
\hline DCAI & $\ddagger 2.37 \mathrm{E}-19$ & $\dagger 1726$. & $\dagger 1.67 \mathrm{E}-21$ & $\dagger 2153$ & $\dagger 1.00 \mathrm{E}-24$ & $\dagger 2800$ & $+8.00 \mathrm{E}-29$ & $\dagger 3600$ & $\dagger 5.00 \mathrm{E}-32$ & $\dagger 4500$ & $\dagger 3.00 E-36$ & $\dagger 6000$ \\
\hline SC & $2.64 \mathrm{E}-18$ & 233. & $1.71 \mathrm{E}-19$ & 463. & $2.10 \mathrm{E}-20$ & 1008. & $3.35 \mathrm{E}-21$ & 2520. & $1.41 \mathrm{E}-23$ & 4591. & $1.72 \mathrm{E}-25$ & 12760. \\
\hline DC & $1.24 \mathrm{E}-19$ & 481. & $1.81 \mathrm{E}-22$ & 951. & $\dagger 1.00 \mathrm{E}-25$ & $\dagger 2040$ & $\dagger 7.00 \mathrm{E}-30$ & † 5095 & $\dagger 5.00 \mathrm{E}-33$ & † 9394 & $\dagger 3.00 E-37$ & $\dagger 25732$ \\
\hline TEX & $1.14 \mathrm{E}-15$ & 7.70 & $1.12 \mathrm{E}-15$ & 7.70 & $8.33 \mathrm{E}-16$ & 7.70 & $4.60 \mathrm{E}-16$ & 7.70 & $2.59 \mathrm{E}-16$ & 7.70 & $1.10 \mathrm{E}-16$ & 7.70 \\
\hline
\end{tabular}

† Extrapolated. $\ddagger$ Significantly improved. $\quad \S$ Corrects typographical error in Ref. [1]. $\quad{ }^{*}$ Includes Born correction. 
Table 12

The singly differential cross section as a function of the ejected electron energy for single ionization in 5000-25000 keV/u 0, $0^{\wedge}+, 0^{\wedge} 2+$ + H_2 collisions.

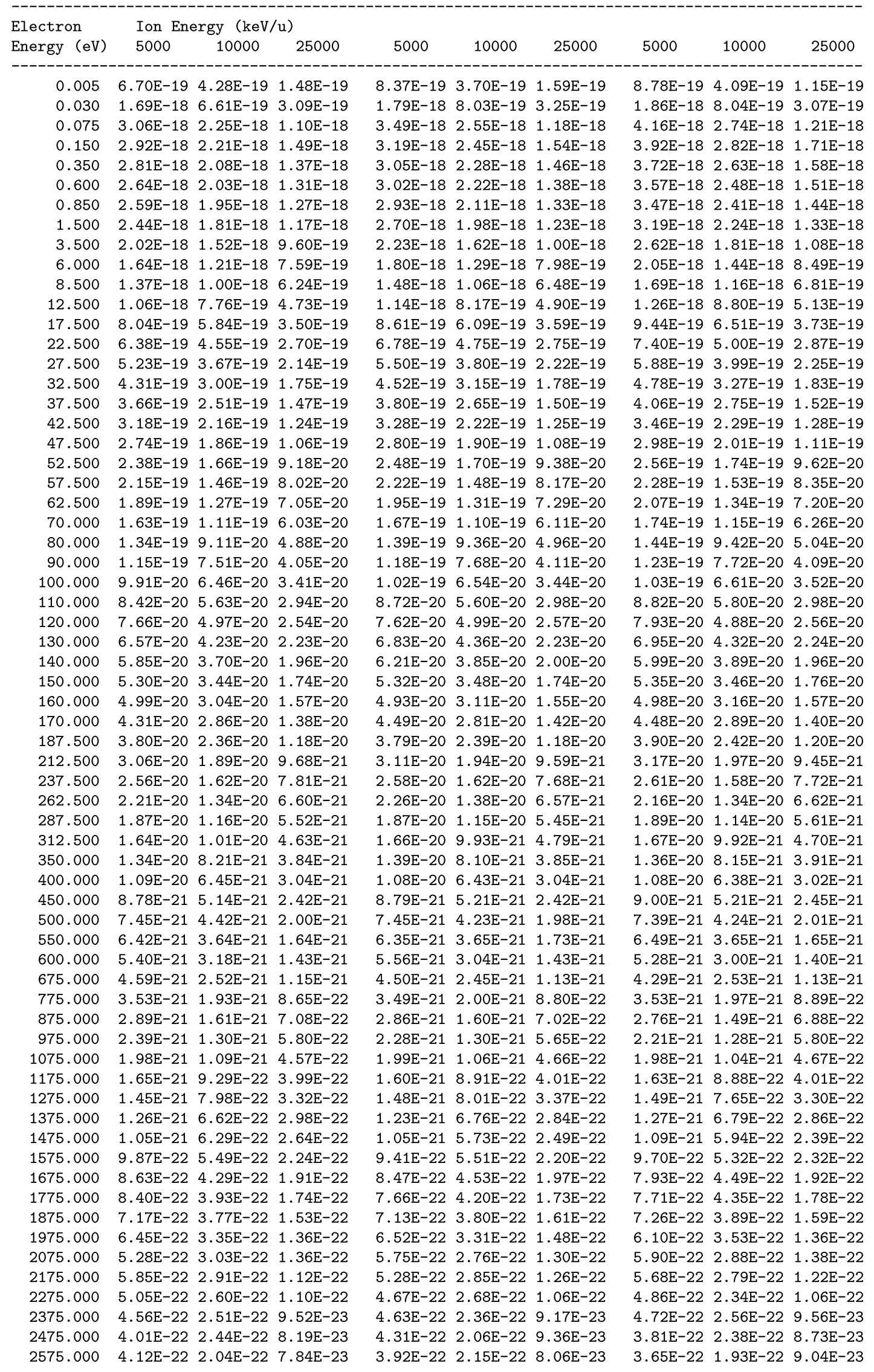


Table 12 (continued)

$2725.000 \quad 3.54 \mathrm{E}-22 \quad 1.68 \mathrm{E}-22 \quad 7.42 \mathrm{E}-23$ $2925.000 \quad 3.13 \mathrm{E}-22 \quad 1.52 \mathrm{E}-22 \quad 6.31 \mathrm{E}-23$ $3125.000 \quad 2.71 \mathrm{E}-22 \quad 1.42 \mathrm{E}-22 \quad 5.96 \mathrm{E}-23$ $3325.000 \quad 2.45 \mathrm{E}-22 \quad 1.24 \mathrm{E}-22 \quad 5.02 \mathrm{E}-23$ $3525.000 \quad 2.26 \mathrm{E}-22 \quad 1.17 \mathrm{E}-22 \quad 4.57 \mathrm{E}-23$ $3725.000 \quad 1.95 \mathrm{E}-22 \quad 1.02 \mathrm{E}-22 \quad 4.45 \mathrm{E}-23$ $3925.000 \quad 1.71 \mathrm{E}-22 \quad 8.17 \mathrm{E}-23 \quad 3.74 \mathrm{E}-23$ $4125.000 \quad 1.62 \mathrm{E}-22 \quad 8.14 \mathrm{E}-23 \quad 3.82 \mathrm{E}-23$ $4325.000 \quad 1.62 \mathrm{E}-22 \quad 6.80 \mathrm{E}-23 \quad 3.12 \mathrm{E}-23$ $4525.000 \quad 1.33 \mathrm{E}-22 \quad 6.39 \mathrm{E}-23 \quad 2.76 \mathrm{E}-23$ $4725.000 \quad 1.22 \mathrm{E}-22 \quad 5.68 \mathrm{E}-23 \quad 2.64 \mathrm{E}-23$ $\begin{array}{llll}4925.000 & 1.31 \mathrm{E}-22 & 5.56 \mathrm{E}-23 & 2.38 \mathrm{E}-23\end{array}$ $5125.000 \quad 1.05 \mathrm{E}-22 \quad 6.10 \mathrm{E}-23 \quad 2.19 \mathrm{E}-23$ $5325.000 \quad 1.03 \mathrm{E}-22 \quad 5.33 \mathrm{E}-23 \quad 2.12 \mathrm{E}-23$ $\begin{array}{llll}5525.000 & 9.11 \mathrm{E}-23 & 4.73 \mathrm{E}-23 & 2.00 \mathrm{E}-23\end{array}$ $5725.000 \quad 8.27 \mathrm{E}-23 \quad 4.50 \mathrm{E}-23 \quad 1.71 \mathrm{E}-23$ $\begin{array}{llll}5925.000 & 7.59 \mathrm{E}-23 & 4.66 \mathrm{E}-23 & 1.72 \mathrm{E}-23\end{array}$ $6125.000 \quad 6.07 \mathrm{E}-23 \quad 3.35 \mathrm{E}-23 \quad 1.58 \mathrm{E}-23$ $6325.000 \quad 6.33 \mathrm{E}-23 \quad 3.70 \mathrm{E}-23 \quad 1.29 \mathrm{E}-23$ $6525.000 \quad 7.22 \mathrm{E}-23 \quad 3.03 \mathrm{E}-23 \quad 1.17 \mathrm{E}-23$ $6875.000 \quad 5.82 \mathrm{E}-23 \quad 3.17 \mathrm{E}-23 \quad 1.28 \mathrm{E}-23$ $\begin{array}{llll}7375.000 & 5.26 \mathrm{E}-23 & 2.62 \mathrm{E}-23 & 1.01 \mathrm{E}-23\end{array}$ $\begin{array}{llll}7875.000 & 4.82 \mathrm{E}-23 & 2.50 \mathrm{E}-23 & 8.40 \mathrm{E}-24\end{array}$ $8375.000 \quad 4.17 \mathrm{E}-23 \quad 2.00 \mathrm{E}-23 \quad 7.36 \mathrm{E}-24$ $8875.000 \quad 3.58 \mathrm{E}-23 \quad 1.80 \mathrm{E}-23 \quad 6.92 \mathrm{E}-24$ $9375.000 \quad 3.08 \mathrm{E}-23 \quad 1.25 \mathrm{E}-23 \quad 6.47 \mathrm{E}-24$ $9875.000 \quad 2.87 \mathrm{E}-23 \quad 1.35 \mathrm{E}-23 \quad 5.81 \mathrm{E}-24$ $\begin{array}{llll}10375.000 & 2.07 \mathrm{E}-23 & 1.29 \mathrm{E}-23 & 5.36 \mathrm{E}-24\end{array}$ $10875.000 \quad 1.09 \mathrm{E}-23 \quad 1.33 \mathrm{E}-23 \quad 4.84 \mathrm{E}-24$ $\begin{array}{llll}11375.000 & 2.93 \mathrm{E}-24 & 1.16 \mathrm{E}-23 & 4.29 \mathrm{E}-24\end{array}$ 11875.000 ------- 9.96E-24 4.66E-24 12375.000 ------- $1.06 \mathrm{E}-23 \quad 4.07 \mathrm{E}-24$ 12875.000 ------ 9.19E-24 3.70E-24 13375.000 ------ 9.71E-24 3.40E-24 $13875.000 \quad------6.39 \mathrm{E}-24 \quad 2.96 \mathrm{E}-24$ 14375.000 ------ 7.53E-24 2.74E-24 14875.000 ------ 7.53E-24 2.48E-24 15375.000 ------ 7.02E-24 $2.44 \mathrm{E}-24$ 15875.000 ------ 6.90E-24 2.33E-24 16375.000 ------- 5.87E-24 2.59E-24 17125.000 ------- $4.53 \mathrm{E}-24 \quad 2.31 \mathrm{E}-24$ 18125.000 ------ 3.90E-24 2.09E-24 19125.000 ------ 3.51E-24 1.72E-24 20125.000 ------ 3.32E-24 1.44E-24 21125.000 ------- 2.30E-24 $1.35 \mathrm{E}-24$ 22125.000 ------- 9.58E-25 $1.41 \mathrm{E}-24$ 23125.000 ------- ------- $1.05 \mathrm{E}-24$ 24125.000 -------- ------- 9.25E-25 25125.000 ------- ------- 9.25E-25 26125.000 -------- -------- 1.09E-24 27125.000 ------- ------- 8.14E-25 28125.000 ------- ------- 7.58E-25 29125.000 ------- ------ 9.62E-25 30125.000 ------- ------ 5.73E-25 31125.000 ------- ------ 6.47E-25 32125.000 ------- ------- 6.47E-25 33125.000 -------- ------- 7.03E-25 34125.000 -------- ------- 4.62E-25 35125.000 -------- ------- 4.62E-25 36125.000 ------- ------- 4.62E-25 37875.000 ------- --- 4.14E-25 40375.000 -------- ------- 3.40E-25 42875.000 ------- ------- 3.11E-25 45375.000 ------- ------ 3.18E-25 47875.000 -------- ------- 2.81E-25 50375.000 ------- ------- 2.00E-25 52875.000 ------- ------- 2.44E-25 55375.000 ------- ------ 5.92E-26
$3.55 \mathrm{E}-22 \quad 1.90 \mathrm{E}-22 \quad 8.08 \mathrm{E}-23$ $2.98 \mathrm{E}-22 \quad 1.62 \mathrm{E}-22 \quad 6.81 \mathrm{E}-23$ $2.81 \mathrm{E}-22 \quad 1.33 \mathrm{E}-22 \quad 6.19 \mathrm{E}-23$ $2.52 \mathrm{E}-22 \quad 1.20 \mathrm{E}-22 \quad 5.20 \mathrm{E}-23$ 2. $24 \mathrm{E}-22 \quad 1.18 \mathrm{E}-22 \quad 4.59 \mathrm{E}-23$ $1.95 \mathrm{E}-22 \quad 9.87 \mathrm{E}-23 \quad 4.09 \mathrm{E}-23$ $\begin{array}{lll}1.77 \mathrm{E}-22 & 9.26 \mathrm{E}-23 \quad 3.82 \mathrm{E}-23\end{array}$ $1.55 \mathrm{E}-22 \quad 8.05 \mathrm{E}-23 \quad 3.55 \mathrm{E}-23$ 1.63E-22 $7.34 \mathrm{E}-23 \quad 3.00 \mathrm{E}-23$ $\begin{array}{lll}1.31 \mathrm{E}-22 & 6.86 \mathrm{E}-23 & 2.82 \mathrm{E}-23\end{array}$ $\begin{array}{lll}1.41 \mathrm{E}-22 & 7.73 \mathrm{E}-23 & 2.38 \mathrm{E}-23\end{array}$ 1. 23E-22 $5.87 \mathrm{E}-23 \quad 2.17 \mathrm{E}-23$ $\begin{array}{ll}1.10 \mathrm{E}-22 & 5.20 \mathrm{E}-23 \\ 2.10 \mathrm{E}-23\end{array}$ $9.89 \mathrm{E}-23 \quad 4.69 \mathrm{E}-23 \quad 1.86 \mathrm{E}-23$ $\begin{array}{lll}7.59 \mathrm{E}-23 & 4.25 \mathrm{E}-23 & 1.82 \mathrm{E}-23\end{array}$ $8.37 \mathrm{E}-23 \quad 4.02 \mathrm{E}-23 \quad 1.86 \mathrm{E}-23$

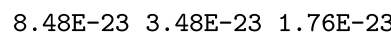
$\begin{array}{llll}7.64 \mathrm{E}-23 & 3.67 \mathrm{E}-23 & 1.43 \mathrm{E}-23\end{array}$ $6.07 \mathrm{E}-23 \quad 2.87 \mathrm{E}-23 \quad 1.68 \mathrm{E}-23$ $6.65 \mathrm{E}-23 \quad 2.91 \mathrm{E}-23 \quad 1.57 \mathrm{E}-23$ $5.65 \mathrm{E}-23 \quad 3.15 \mathrm{E}-23 \quad 1.36 \mathrm{E}-23$ $5.40 \mathrm{E}-23 \quad 3.00 \mathrm{E}-23 \quad 1.07 \mathrm{E}-23$ $5.21 \mathrm{E}-23 \quad 1.98 \mathrm{E}-23 \quad 9.39 \mathrm{E}-24$ 4. 04E-23 $2.35 \mathrm{E}-23 \quad 8.99 \mathrm{E}-24$ $4.19 \mathrm{E}-23 \quad 1.78 \mathrm{E}-23 \quad 7.95 \mathrm{E}-24$ 3. 52E-23 1.61E-23 6.36E-24 $2.99 \mathrm{E}-23 \quad 1.58 \mathrm{E}-23 \quad 5.21 \mathrm{E}-24$ $2.39 \mathrm{E}-23 \quad 1.40 \mathrm{E}-23 \quad 5.84 \mathrm{E}-24$ 1. $24 \mathrm{E}-23 \quad 1.52 \mathrm{E}-23 \quad 4.25 \mathrm{E}-24$ 2. 30E-24 9.58E-24 3.99E-24 ------ $1.11 \mathrm{E}-23 \quad 4.25 \mathrm{E}-24$ ------ 8.56E-24 4.07E-24 ------- 8.56E-24 3.55E-24 ------- 9.58E-24 3.25E-24 ------ 6.26E-24 3.48E-24 ------ 5.24E-24 3.29E-24 ------ 6.26E-24 2.26E-24 ------ 5.11E-24 2.26E-24 ------- 6.64E-24 1.81E-24 ------ 6.26E-24 2.40E-24 ------- 5.43E-24 2.18E-24 ------ 5.04E-24 1.72E-24 ------- 4.73E-24 1.79E-24 ------ 3.45E-24 1.65E-24 ------- 2.49E-24 1.41E-24 ------- 7.02E-25 1.11E-24

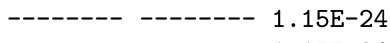
-------- -------- 1.15E-24 ------ ------ 9.80E-25 ------- ------- 7.40E-25 ------- ------- 9.25E-25 ------- ------ 6.29E-25 ------ ------ $7.77 \mathrm{E}-25$ --o--- ------ 7.40E-25 ------- ------ 5.73E-25 ------- ------ 6.10E-25 -------- -------- 4.62E-25 ------- ------- 5.18E-25 ------- ------- 4.07E-25 ------ ------ $6.84 \mathrm{E}-25$ ------- ------ 3.70E-25 ------- ------- 3.92E-25 ------- ------- 2.74E-25 ---c-- ------- 1.92E-25 ------ ------ 2.29E-25 -------- -------- 3.11E-25 ------ ------ 2.00E-25

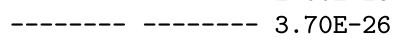

$3.51 \mathrm{E}-22 \quad 1.90 \mathrm{E}-22 \quad 6.89 \mathrm{E}-23$ $\begin{array}{ll}3.10 \mathrm{E}-22 & 1.54 \mathrm{E}-22 \\ 6.58 \mathrm{E}-23\end{array}$ $2.52 \mathrm{E}-22 \quad 1.49 \mathrm{E}-22 \quad 5.59 \mathrm{E}-23$ $2.38 \mathrm{E}-22 \quad 1.11 \mathrm{E}-22 \quad 5.41 \mathrm{E}-23$ 2. 14E-22 1.15E-22 4.58E-23 $1.75 \mathrm{E}-22 \quad 1.01 \mathrm{E}-22 \quad 4.08 \mathrm{E}-23$ $\begin{array}{lll}1.69 \mathrm{E}-22 & 9.16 \mathrm{E}-23 \quad 3.81 \mathrm{E}-23\end{array}$ $\begin{array}{lll}1.60 \mathrm{E}-22 & 8.40 \mathrm{E}-23 & 3.52 \mathrm{E}-23\end{array}$ $\begin{array}{lll}1.44 \mathrm{E}-22 & 7.36 \mathrm{E}-23 \quad 2.88 \mathrm{E}-23\end{array}$ $\begin{array}{lll}1.27 \mathrm{E}-22 & 7.15 \mathrm{E}-23 & 2.95 \mathrm{E}-23\end{array}$ $\begin{array}{ll}1.24 \mathrm{E}-22 & 6.46 \mathrm{E}-23 \quad 2.86 \mathrm{E}-23\end{array}$ $\begin{array}{lll}1.17 \mathrm{E}-22 & 6.79 \mathrm{E}-23 & 2.38 \mathrm{E}-23\end{array}$ $\begin{array}{lll}1.16 \mathrm{E}-22 & 5.03 \mathrm{E}-23 & 2.30 \mathrm{E}-23\end{array}$ $9.23 \mathrm{E}-23 \quad 4.81 \mathrm{E}-23 \quad 2.12 \mathrm{E}-23$ $9.00 \mathrm{E}-23 \quad 4.27 \mathrm{E}-23 \quad 1.94 \mathrm{E}-23$ $9.52 \mathrm{E}-23 \quad 4.45 \mathrm{E}-23 \quad 1.94 \mathrm{E}-23$ $\begin{array}{lll}7.69 \mathrm{E}-23 & 3.41 \mathrm{E}-23 & 1.59 \mathrm{E}-23\end{array}$ $6.95 \mathrm{E}-23 \quad 3.91 \mathrm{E}-23 \quad 1.37 \mathrm{E}-23$ 6. 55E $-23 \quad 3.59 \mathrm{E}-23 \quad 1.54 \mathrm{E}-23$ 6. $27 \mathrm{E}-23 \quad 3.45 \mathrm{E}-23 \quad 1.36 \mathrm{E}-23$ 5.72E-23 $2.90 \mathrm{E}-23 \quad 1.22 \mathrm{E}-23$ $5.11 \mathrm{E}-23 \quad 2.66 \mathrm{E}-23 \quad 1.11 \mathrm{E}-23$ $\begin{array}{lll}4.67 \mathrm{E}-23 & 2.18 \mathrm{E}-23 & 8.94 \mathrm{E}-24\end{array}$ 3. $76 \mathrm{E}-23 \quad 2.31 \mathrm{E}-23 \quad 8.98 \mathrm{E}-24$ $\begin{array}{llll}3.60 \mathrm{E}-23 & 2.14 \mathrm{E}-23 & 6.89 \mathrm{E}-24\end{array}$ $3.78 \mathrm{E}-23 \quad 1.57 \mathrm{E}-23 \quad 6.65 \mathrm{E}-24$ $2.83 \mathrm{E}-23 \quad 1.41 \mathrm{E}-23 \quad 5.74 \mathrm{E}-24$ $2.42 \mathrm{E}-23 \quad 1.36 \mathrm{E}-23 \quad 5.11 \mathrm{E}-24$ 1. $14 \mathrm{E}-23 \quad 1.38 \mathrm{E}-23 \quad 5.07 \mathrm{E}-24$ $2.05 \mathrm{E}-24 \quad 1.13 \mathrm{E}-23 \quad 4.55 \mathrm{E}-24$ ------ $1.08 \mathrm{E}-23 \quad 3.72 \mathrm{E}-24$ ------- 1.01E-23 4.04E-24 ------ $7.04 \mathrm{E}-24 \quad 3.21 \mathrm{E}-24$ ------ $7.47 \mathrm{E}-24$ 3.13E-24 ------- 8.76E-24 3.40E-24 ------ 5.03E-24 3.21E-24 ------ 8.19E-24 2.30E-24 ------- 5.75E-24 2.73E-24 ------ 6.61E-24 2.06E-24 ------- 4.60E-24 2.14E-24 ------- 5.53E-24 2.14E-24 ------ 4.45E-24 2.04E-24 ------- 4.45E-24 1.66E-24 ------ 4.09E-24 1.50E-24 ------- 2.80E-24 1.17E-24 ------- 6.46E-25 $1.09 \mathrm{E}-24$

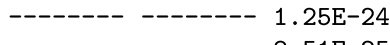
------- ------- 8.51E-25 ------ ------ $1.01 \mathrm{E}-24$ ------ ------ 8.11E-25 ------- ------- 7.52E-25 ------ ------ 7.12E-25 ------ ------ 6.53E-25 ------- ------ 5.34E-25 -------- -------- 5.94E-25 ------ ------ 5.74E-25 ------- ------- 5.15E-25 ------- ------- 4.35E-25 ------ ------- 5.54E-25 ------- ------- 3.17E-25 -------- ------- 4.67E-25 ------- ------- 3.17E-25 ------ ------- 3.64E-25 -------- -------- $2.77 \mathrm{E}-25$ ------- ------- 2.61E-25 ------- ------- $2.30 \mathrm{E}-25$ ------- ------- 2.14E-25 ------ ------ $4.75 \mathrm{E}-26$ 
Table 13

The singly differential cross section as a function of the ejected electron energy for single ionization in $5000-25000 \mathrm{keV} / \mathrm{u} \mathrm{O`} 3+, 0^{\wedge} 4+$, 0^5++ H_2 collisions.

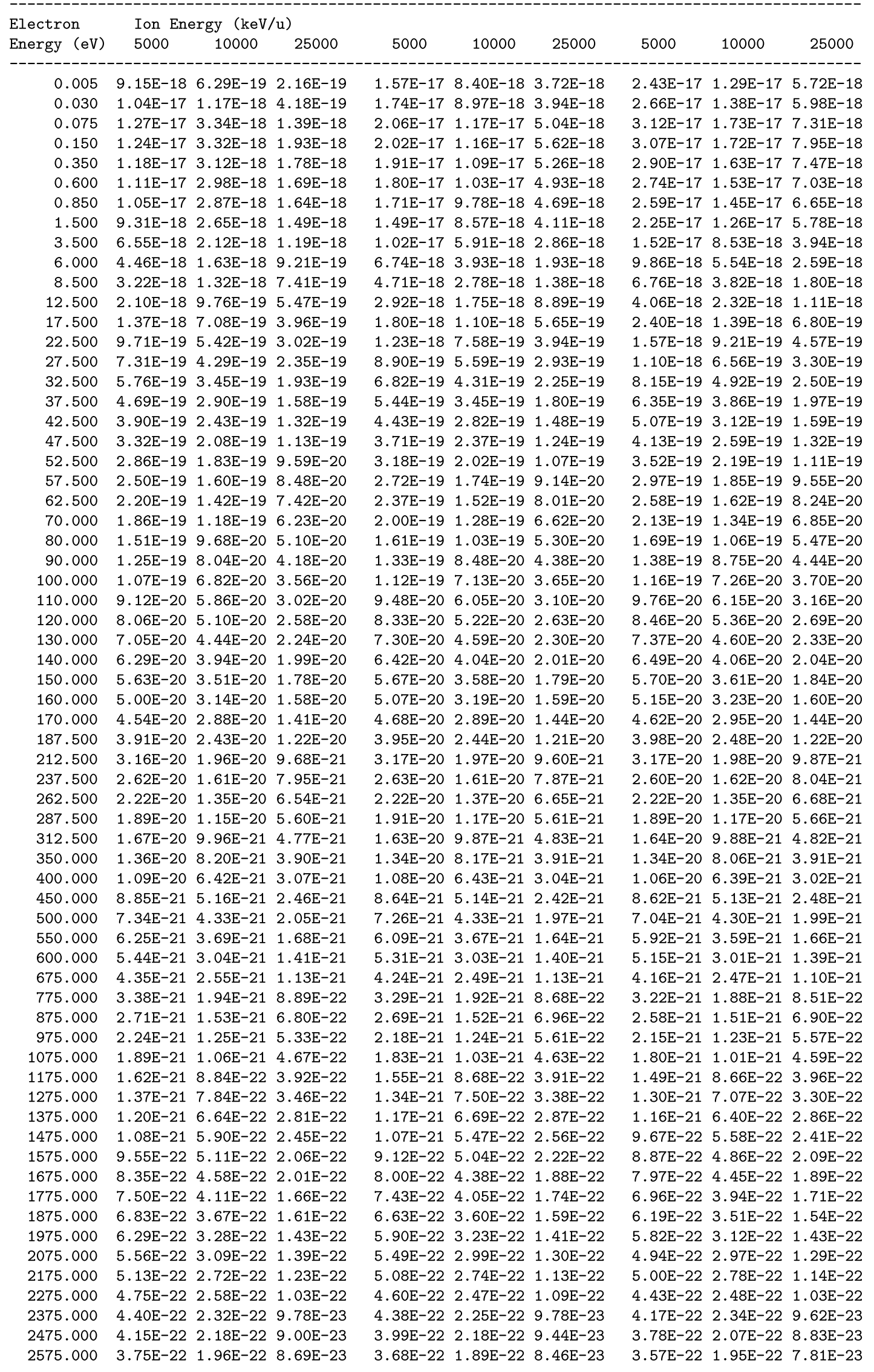


Table 13 (continued)

$2725.000 \quad 3.33 \mathrm{E}-22 \quad 1.82 \mathrm{E}-22 \quad 7.46 \mathrm{E}-23$ $2925.000 \quad 3.05 \mathrm{E}-22 \quad 1.57 \mathrm{E}-22 \quad 6.31 \mathrm{E}-23$ $3125.000 \quad 2.65 \mathrm{E}-22 \quad 1.40 \mathrm{E}-22 \quad 6.08 \mathrm{E}-23$ $3325.000 \quad 2.30 \mathrm{E}-22 \quad 1.16 \mathrm{E}-22 \quad 4.99 \mathrm{E}-23$ $3525.000 \quad 2.09 \mathrm{E}-22 \quad 1.09 \mathrm{E}-22 \quad 4.17 \mathrm{E}-23$ $3725.000 \quad 1.88 \mathrm{E}-22 \quad 1.03 \mathrm{E}-22 \quad 4.13 \mathrm{E}-23$ $3925.000 \quad 1.72 \mathrm{E}-22 \quad 8.80 \mathrm{E}-23 \quad 3.78 \mathrm{E}-23$ $4125.000 \quad 1.55 \mathrm{E}-22 \quad 8.00 \mathrm{E}-23 \quad 3.54 \mathrm{E}-23$ $4325.000 \quad 1.45 \mathrm{E}-22 \quad 7.11 \mathrm{E}-23 \quad 3.41 \mathrm{E}-23$ $\begin{array}{lllll}4525.000 & 1.34 \mathrm{E}-22 & 6.61 \mathrm{E}-23 & 2.78 \mathrm{E}-23\end{array}$ $4725.000 \quad 1.21 \mathrm{E}-22 \quad 5.96 \mathrm{E}-23 \quad 2.55 \mathrm{E}-23$ $4925.000 \quad 1.10 \mathrm{E}-22 \quad 5.66 \mathrm{E}-23 \quad 2.54 \mathrm{E}-23$ $\begin{array}{llll}5125.000 & 1.02 \mathrm{E}-22 & 5.37 \mathrm{E}-23 & 2.23 \mathrm{E}-23\end{array}$ $\begin{array}{lllll}5325.000 & 9.47 \mathrm{E}-23 & 4.87 \mathrm{E}-23 & 2.23 \mathrm{E}-23\end{array}$ $5525.000 \quad 8.92 \mathrm{E}-23 \quad 4.57 \mathrm{E}-23 \quad 1.94 \mathrm{E}-23$ $5725.000 \quad 8.13 \mathrm{E}-23 \quad 4.41 \mathrm{E}-23 \quad 1.83 \mathrm{E}-23$ $\begin{array}{llll}5925.000 & 8.07 \mathrm{E}-23 & 4.08 \mathrm{E}-23 & 1.43 \mathrm{E}-23\end{array}$ $6125.000 \quad 7.56 \mathrm{E}-23 \quad 3.85 \mathrm{E}-23 \quad 1.43 \mathrm{E}-23$ $6325.000 \quad 6.91 \mathrm{E}-23 \quad 3.71 \mathrm{E}-23 \quad 1.52 \mathrm{E}-23$ $6525.000 \quad 7.09 \mathrm{E}-23 \quad 3.29 \mathrm{E}-23 \quad 1.63 \mathrm{E}-23$ $6875.000 \quad 5.99 \mathrm{E}-23 \quad 3.02 \mathrm{E}-23 \quad 1.20 \mathrm{E}-23$ $\begin{array}{llll}7375.000 & 5.19 \mathrm{E}-23 & 2.53 \mathrm{E}-23 & 1.16 \mathrm{E}-23\end{array}$ $\begin{array}{llll}7875.000 & 4.52 \mathrm{E}-23 & 2.18 \mathrm{E}-23 & 9.54 \mathrm{E}-24\end{array}$ $8375.000 \quad 4.15 \mathrm{E}-23 \quad 1.89 \mathrm{E}-23 \quad 8.47 \mathrm{E}-24$ $8875.000 \quad 3.26 \mathrm{E}-23 \quad 1.83 \mathrm{E}-23 \quad 7.05 \mathrm{E}-24$ $9375.000 \quad 3.35 \mathrm{E}-23 \quad 1.59 \mathrm{E}-23 \quad 7.08 \mathrm{E}-24$ $9875.000 \quad 2.75 \mathrm{E}-23 \quad 1.39 \mathrm{E}-23 \quad 6.10 \mathrm{E}-24$ $\begin{array}{llll}10375.000 & 2.26 \mathrm{E}-23 & 1.34 \mathrm{E}-23 & 6.65 \mathrm{E}-24\end{array}$ $10875.000 \quad 1.22 \mathrm{E}-23 \quad 1.20 \mathrm{E}-23 \quad 4.99 \mathrm{E}-24$ $11375.000 \quad 3.08 \mathrm{E}-24 \quad 1.14 \mathrm{E}-23 \quad 4.51 \mathrm{E}-24$ $11875.000 \quad 4.41 \mathrm{E}-25 \quad 9.64 \mathrm{E}-24 \quad 3.72 \mathrm{E}-24$ 12375.000 ------ $8.71 \mathrm{E}-24 \quad 3.29 \mathrm{E}-24$ 12875.000 ------ 8.74E-24 3.17E-24 13375.000 ------ $8.08 \mathrm{E}-24 \quad 3.01 \mathrm{E}-24$ $13875.000 \quad------7.33 \mathrm{E}-24 \quad 3.84 \mathrm{E}-24$ 14375.000 ------ 7.45E-24 2.65E-24 14875.000 ------ 5.88E-24 2.45E-24 15375.000 ------ 5.37E-24 3.17E-24 15875.000 ------ 5.43E-24 $1.90 \mathrm{E}-24$ 16375.000 ------- 4.95E-24 $1.98 \mathrm{E}-24$ 17125.000 ------- $4.53 \mathrm{E}-24 \quad 2.12 \mathrm{E}-24$ 18125.000 ------- $4.26 \mathrm{E}-24 \quad 1.54 \mathrm{E}-24$ 19125.000 ------ 4.34E-24 1.84E-24 20125.000 ------ 3.26E-24 1.54E-24 21125.000 ------- 2.34E-24 $1.19 \mathrm{E}-24$ 22125.000 ------- 9.01E-25 $1.37 \mathrm{E}-24$ 23125.000 ------- ------- 1.33E-24 24125.000 -------- ------- 8.91E-25 25125.000 ------- ------ 8.51E-25 26125.000 -------- -------- 8.71E-25 27125.000 ------ ------ 6.93E-25 28125.000 ------- ------- 5.34E-25 29125.000 ------- ------- 4.95E-25 30125.000 -------- ------- 5.74E-25 31125.000 ------- ------- 4.75E-25 32125.000 ------- ------- 7.32E-25 33125.000 ------- ------- 5.74E-25 34125.000 ------- ------- 3.56E-25 35125.000 ------- ------- 3.56E-25 36125.000 ------- ------- 4.75E-25 37875.000 ------- --- 4.51E-25 40375.000 ------- ------- 3.64E-25 42875.000 ------- ------- 3.72E-25 45375.000 ------- ------- 2.45E-25 47875.000 -------- ------- 2.14E-25 50375.000 ------- ------- 2.22E-25 52875.000 -------- ------ $1.82 \mathrm{E}-25$ 55375.000 ------- ------ 3.96E-26
$3.46 \mathrm{E}-22 \quad 1.76 \mathrm{E}-22 \quad 7.32 \mathrm{E}-23$ $2.80 \mathrm{E}-22 \quad 1.52 \mathrm{E}-22 \quad 6.71 \mathrm{E}-23$ $2.58 \mathrm{E}-22 \quad 1.38 \mathrm{E}-22 \quad 5.69 \mathrm{E}-23$ $2.26 \mathrm{E}-22 \quad 1.19 \mathrm{E}-22 \quad 4.95 \mathrm{E}-23$ $2.02 \mathrm{E}-22 \quad 1.11 \mathrm{E}-22 \quad 4.59 \mathrm{E}-23$ $1.85 \mathrm{E}-22 \quad 9.59 \mathrm{E}-23 \quad 4.13 \mathrm{E}-23$ $1.64 \mathrm{E}-22 \quad 8.53 \mathrm{E}-23 \quad 3.82 \mathrm{E}-23$ $1.53 \mathrm{E}-22 \quad 7.67 \mathrm{E}-23 \quad 3.08 \mathrm{E}-23$ 1. 39E-22 $7.19 \mathrm{E}-23 \quad 3.11 \mathrm{E}-23$ $1.26 \mathrm{E}-22 \quad 6.87 \mathrm{E}-23 \quad 2.83 \mathrm{E}-23$ $\begin{array}{lll}1.14 \mathrm{E}-22 & 6.16 \mathrm{E}-23 & 2.62 \mathrm{E}-23\end{array}$ $1.11 \mathrm{E}-22 \quad 5.58 \mathrm{E}-23 \quad 2.37 \mathrm{E}-23$ 9.61E-23 5.00E-23 2.17E-23 8.95E-23 4.64E-23 1.93E-23 $8.77 \mathrm{E}-23 \quad 4.56 \mathrm{E}-23 \quad 1.90 \mathrm{E}-23$ 8. $26 \mathrm{E}-23 \quad 4.24 \mathrm{E}-23 \quad 1.78 \mathrm{E}-23$ 7.37E-23 4.17E-23 1.60E-23 $6.84 \mathrm{E}-23 \quad 3.91 \mathrm{E}-23 \quad 1.41 \mathrm{E}-23$ $6.61 \mathrm{E}-23 \quad 3.24 \mathrm{E}-23 \quad 1.55 \mathrm{E}-23$ $5.97 \mathrm{E}-23 \quad 3.04 \mathrm{E}-23 \quad 1.31 \mathrm{E}-23$ $5.82 \mathrm{E}-23 \quad 2.93 \mathrm{E}-23 \quad 1.21 \mathrm{E}-23$ $5.05 \mathrm{E}-23 \quad 2.52 \mathrm{E}-23 \quad 1.10 \mathrm{E}-23$ 4. 46E-23 2.16E-23 8.64E-24 3. 97E-23 $2.01 \mathrm{E}-23 \quad 8.03 \mathrm{E}-24$ 3. 63E-23 $1.75 \mathrm{E}-23 \quad 7.16 \mathrm{E}-24$ 3. 03E-23 1.69E-23 7.20E-24 $2.73 \mathrm{E}-23 \quad 1.39 \mathrm{E}-23 \quad 6.13 \mathrm{E}-24$ $2.18 \mathrm{E}-23 \quad 1.27 \mathrm{E}-23 \quad 5.41 \mathrm{E}-24$ $1.06 \mathrm{E}-23 \quad 1.17 \mathrm{E}-23 \quad 5.24 \mathrm{E}-24$ 2. 33E-24 1.09E-23 4.53E-24 $3.81 \mathrm{E}-25 \quad 9.77 \mathrm{E}-24 \quad 4.23 \mathrm{E}-24$ ------ 8.35E-24 3.51E-24 ------- 8.04E-24 3.78E-24 ------- 7.12E-24 3.17E-24 ------ 6.98E-24 3.02E-24 ------ 7.35E-24 3.10E-24 ------ 6.16E-24 2.49E-24 ----- 5.33E-24 2.06E-24 ------- 5.64E-24 2.33E-24 ------ 5.50E-24 2.18E-24 ------- 4.55E-24 1.96E-24 ------ 4.08E-24 $1.72 \mathrm{E}-24$ ------ 3.85E-24 1.51E-24 ------ 3.39E-24 1.34E-24 ------- 2.54E-24 1.41E-24 ------- $7.12 \mathrm{E}-25 \quad 1.11 \mathrm{E}-24$ ------- $1.42 \mathrm{E}-25 \quad 1.01 \mathrm{E}-24$ -------- -------- 9.58E-25 ------- ------- 9.74E-25 ------- ------ 9.10E-25 ------ ------ 7.76E-25 ------- ------- 8.15E-25 ------ ----- 6.57E-25 ------ ----- 6.97E-25 ------- ------ 4.51E-25 ------- ------- 5.78E-25 ------- ------- 5.46E-25 ------- ------ 5.07E-25 ------ ------ 3.88E-25 ------ ------ $4.99 \mathrm{E}-25$ ------- ------- 3.96E-25 ------- ------- 3.80E-25 ------- ------- 3.39E-25 ------ ------ 2.85E-25 ------- ------ 2.98E-25 ------- ------- 2.28E-25 ------- ------- $1.52 \mathrm{E}-25$ ------ ----- $3.48 \mathrm{E}-26$
3.25E-22 $1.67 \mathrm{E}-22 \quad 7.23 \mathrm{E}-23$ $2.61 \mathrm{E}-22 \quad 1.52 \mathrm{E}-22 \quad 6.95 \mathrm{E}-23$ $2.41 \mathrm{E}-22 \quad 1.29 \mathrm{E}-22 \quad 5.67 \mathrm{E}-23$ $2.05 \mathrm{E}-22 \quad 1.16 \mathrm{E}-22 \quad 5.07 \mathrm{E}-23$ $2.07 \mathrm{E}-22 \quad 1.04 \mathrm{E}-22 \quad 4.61 \mathrm{E}-23$ $1.72 \mathrm{E}-22 \quad 9.57 \mathrm{E}-23 \quad 4.06 \mathrm{E}-23$ 1.57E-22 $8.45 \mathrm{E}-23 \quad 3.71 \mathrm{E}-23$ $1.50 \mathrm{E}-22 \quad 7.64 \mathrm{E}-23 \quad 3.36 \mathrm{E}-23$ $\begin{array}{lll}1.27 \mathrm{E}-22 & 6.98 \mathrm{E}-23 & 2.75 \mathrm{E}-23\end{array}$ $\begin{array}{lll}1.23 \mathrm{E}-22 & 6.42 \mathrm{E}-23 & 2.66 \mathrm{E}-23\end{array}$ $\begin{array}{ll}1.13 \mathrm{E}-22 & 5.79 \mathrm{E}-23 \quad 2.55 \mathrm{E}-23\end{array}$ \begin{tabular}{l}
$9.65 \mathrm{E}-23 \quad 5.62 \mathrm{E}-23 \quad 2.38 \mathrm{E}-23$ \\
\hline
\end{tabular} $9.60 \mathrm{E}-23 \quad 4.87 \mathrm{E}-23 \quad 2.13 \mathrm{E}-23$ $9.04 \mathrm{E}-23 \quad 4.75 \mathrm{E}-23 \quad 2.33 \mathrm{E}-23$

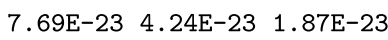

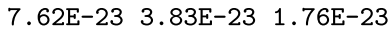

$\begin{array}{lll}7.44 \mathrm{E}-23 & 3.95 \mathrm{E}-23 & 1.48 \mathrm{E}-23\end{array}$

$6.45 \mathrm{E}-23 \quad 3.53 \mathrm{E}-23 \quad 1.58 \mathrm{E}-23$

6. $31 \mathrm{E}-23 \quad 3.34 \mathrm{E}-23 \quad 1.47 \mathrm{E}-23$

6. 64E-23 3.02E-23 $1.43 \mathrm{E}-23$

5. 32E-23 2.90E-23 $1.24 \mathrm{E}-23$

$4.88 \mathrm{E}-23 \quad 2.58 \mathrm{E}-23 \quad 9.85 \mathrm{E}-24$

$\begin{array}{lll}4.32 \mathrm{E}-23 & 2.25 \mathrm{E}-23 & 9.16 \mathrm{E}-24\end{array}$

3. 52E-23 $1.95 \mathrm{E}-23 \quad 8.08 \mathrm{E}-24$

$3.41 \mathrm{E}-23 \quad 1.67 \mathrm{E}-23 \quad 6.44 \mathrm{E}-24$

$2.80 \mathrm{E}-23 \quad 1.62 \mathrm{E}-23 \quad 6.51 \mathrm{E}-24$

$2.44 \mathrm{E}-23 \quad 1.42 \mathrm{E}-23 \quad 5.69 \mathrm{E}-24$

$2.04 \mathrm{E}-23 \quad 1.15 \mathrm{E}-23 \quad 5.00 \mathrm{E}-24$

$9.93 \mathrm{E}-24 \quad 1.16 \mathrm{E}-23 \quad 4.87 \mathrm{E}-24$ $2.30 \mathrm{E}-24 \quad 1.03 \mathrm{E}-23 \quad 4.93 \mathrm{E}-24$ ------- 8.94E-24 4.20E-24 ------ 8.98E-24 4.40E-24 ------- 8.58E-24 3.32E-24 ------ 7.61E-24 3.21E-24 ------ $7.10 \mathrm{E}-24 \quad 3.25 \mathrm{E}-24$ ------ 6.51E-24 2.87E-24 ------ 6.21E-24 1.87E-24 ------ 5.91E-24 2.05E-24 ------ 6.25E-24 2.39E-24 ------ 5.43E-24 2.05E-24 ------- 4.36E-24 2.01E-24 ------- 4.75E-24 1.84E-24 ------- 3.22E-24 1.58E-24 ------ 3.14E-24 1.51E-24 ------ 2.68E-24 1.30E-24 ------- 7.76E-25 $1.08 \mathrm{E}-24$ ------- ------- $1.14 \mathrm{E}-24$ ------- ------- 9.70E-25 ------- ------- 9.37E-25 ------ ------ 7.97E-25 -------- -------- 8.62E-25 ------ ------ 6.79E-25 ------- ------ 6.57E-25 ------- ------ 5.71E-25 -------- -------- $6.46 \mathrm{E}-25$ ------ ------ 5.60E-25 -------- -------- 5.71E-25 -------- ------- 4.63E-25 -------- ------- 4.42E-25 ------ ------ 5.17E-25 ------- ------- 3.71E-25 ------- ------- 3.58E-25 ------- ------- 2.89E-25 ------- ------- 2.20E-25 ------- ------ 2.97E-25 ------- ------- 2.15E-25 -------- ------- $1.38 \mathrm{E}-25$ ------ ------ 2.15E-26 
Table 14

The singly differential cross section as a function of the ejected electron energy for single ionization in 5000-25000 keV/u 0^6+, 0^7+, 0^8++ H_2 collisions.

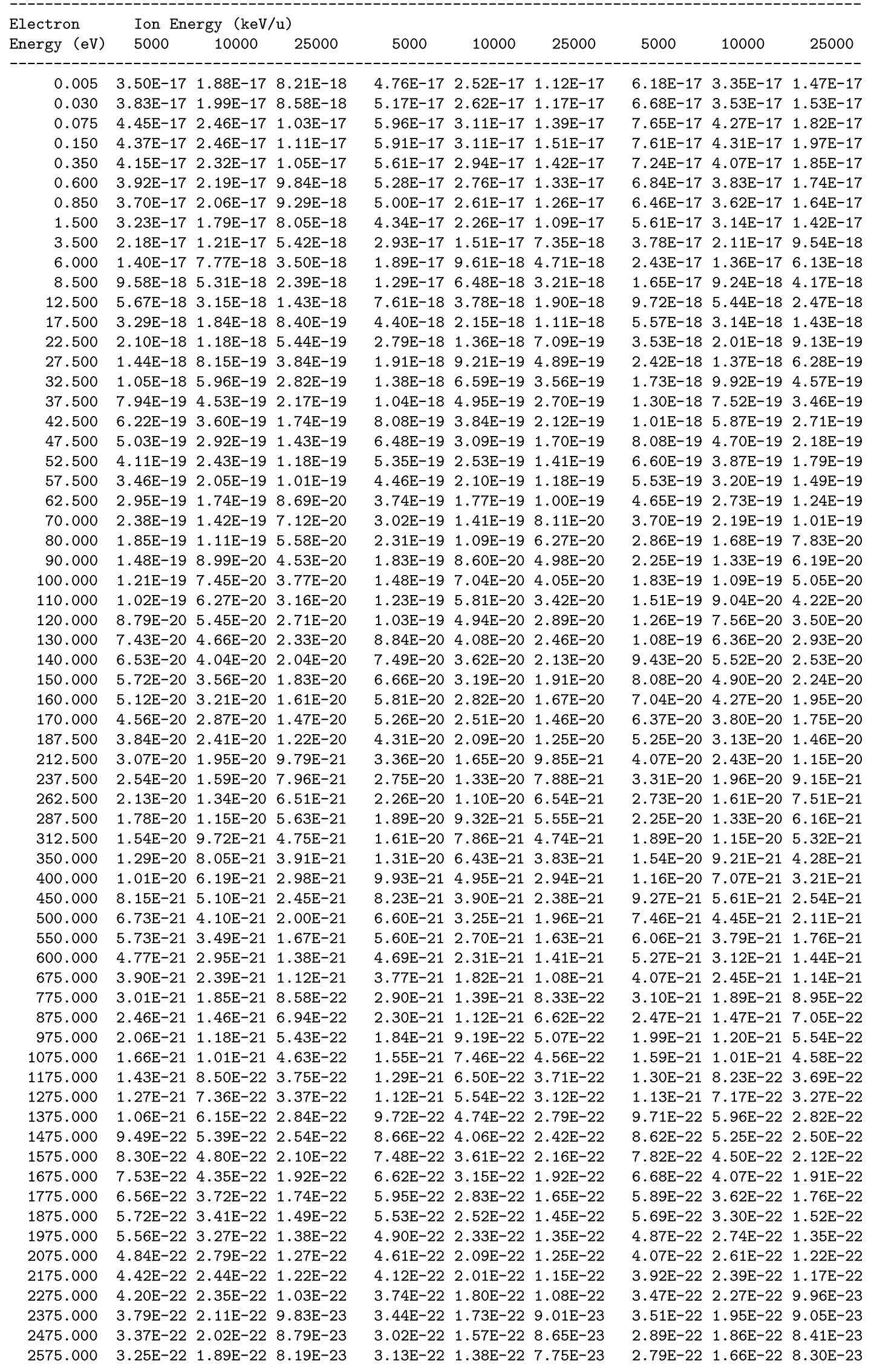


Table 14 (continued)

$2725.000 \quad 3.05 \mathrm{E}-22 \quad 1.67 \mathrm{E}-22 \quad 7.22 \mathrm{E}-23$ $2925.000 \quad 2.64 \mathrm{E}-22 \quad 1.52 \mathrm{E}-22 \quad 6.74 \mathrm{E}-23$ $3125.000 \quad 2.26 \mathrm{E}-22 \quad 1.24 \mathrm{E}-22 \quad 5.68 \mathrm{E}-23$ $3325.000 \quad 2.11 \mathrm{E}-22 \quad 1.11 \mathrm{E}-22 \quad 5.09 \mathrm{E}-23$ $3525.000 \quad 1.78 \mathrm{E}-22 \quad 1.04 \mathrm{E}-22 \quad 4.59 \mathrm{E}-23$ $3725.000 \quad 1.76 \mathrm{E}-22 \quad 8.93 \mathrm{E}-23 \quad 3.70 \mathrm{E}-23$ $3925.000 \quad 1.50 \mathrm{E}-22 \quad 8.37 \mathrm{E}-23 \quad 3.66 \mathrm{E}-23$ $4125.000 \quad 1.31 \mathrm{E}-22 \quad 6.92 \mathrm{E}-23 \quad 3.19 \mathrm{E}-23$ $4325.000 \quad 1.16 \mathrm{E}-22 \quad 7.21 \mathrm{E}-23 \quad 2.95 \mathrm{E}-23$ $4525.000 \quad 1.17 \mathrm{E}-22 \quad 6.45 \mathrm{E}-23 \quad 2.87 \mathrm{E}-23$ $\begin{array}{llll}4725.000 & 1.06 \mathrm{E}-22 & 5.88 \mathrm{E}-23 & 2.68 \mathrm{E}-23\end{array}$ $\begin{array}{lllll}4925.000 & 9.29 \mathrm{E}-23 & 5.44 \mathrm{E}-23 & 2.28 \mathrm{E}-23\end{array}$ $\begin{array}{llll}5125.000 & 8.34 \mathrm{E}-23 & 4.86 \mathrm{E}-23 & 2.18 \mathrm{E}-23\end{array}$ $5325.000 \quad 8.08 \mathrm{E}-23 \quad 4.23 \mathrm{E}-23 \quad 1.99 \mathrm{E}-23$ $5525.000 \quad 7.44 \mathrm{E}-23 \quad 4.13 \mathrm{E}-23 \quad 1.69 \mathrm{E}-23$ $5725.000 \quad 7.53 \mathrm{E}-23 \quad 3.83 \mathrm{E}-23 \quad 1.68 \mathrm{E}-23$ $5925.000 \quad 6.93 \mathrm{E}-23 \quad 3.64 \mathrm{E}-23 \quad 1.55 \mathrm{E}-23$ $6125.000 \quad 6.25 \mathrm{E}-23 \quad 3.27 \mathrm{E}-23 \quad 1.57 \mathrm{E}-23$ $6325.000 \quad 6.39 \mathrm{E}-23 \quad 3.15 \mathrm{E}-23 \quad 1.37 \mathrm{E}-23$ $6525.000 \quad 5.26 \mathrm{E}-23 \quad 3.19 \mathrm{E}-23 \quad 1.37 \mathrm{E}-23$ $6875.000 \quad 4.93 \mathrm{E}-23 \quad 2.74 \mathrm{E}-23 \quad 1.20 \mathrm{E}-23$ $\begin{array}{llll}7375.000 & 4.38 \mathrm{E}-23 & 2.40 \mathrm{E}-23 & 9.96 \mathrm{E}-24\end{array}$ $\begin{array}{llll}7875.000 & 3.93 \mathrm{E}-23 & 2.10 \mathrm{E}-23 & 8.38 \mathrm{E}-24\end{array}$ $8375.000 \quad 3.34 \mathrm{E}-23 \quad 1.75 \mathrm{E}-23 \quad 8.12 \mathrm{E}-24$ $8875.000 \quad 3.10 \mathrm{E}-23 \quad 1.55 \mathrm{E}-23 \quad 7.41 \mathrm{E}-24$ $9375.000 \quad 2.81 \mathrm{E}-23 \quad 1.54 \mathrm{E}-23 \quad 5.99 \mathrm{E}-24$ $9875.000 \quad 2.43 \mathrm{E}-23 \quad 1.26 \mathrm{E}-23 \quad 5.97 \mathrm{E}-24$ $10375.000 \quad 1.85 \mathrm{E}-23 \quad 1.17 \mathrm{E}-23 \quad 5.09 \mathrm{E}-24$ $10875.000 \quad 9.85 \mathrm{E}-24 \quad 1.09 \mathrm{E}-23 \quad 4.85 \mathrm{E}-24$ $11375.000 \quad 1.90 \mathrm{E}-24 \quad 9.39 \mathrm{E}-24 \quad 4.70 \mathrm{E}-24$ 11875.000 ------- 9.03E-24 3.90E-24 12375.000 ------- 8.90E-24 3.66E-24 12875.000 ------ 7.93E-24 3.32E-24 13375.000 ------ $7.56 \mathrm{E}-24 \quad 3.23 \mathrm{E}-24$ 13875.000 ------ 6.79E-24 3.04E-24 14375.000 ------ 5.33E-24 2.52E-24 $14875.000 \quad-----5.61 \mathrm{E}-24 \quad 2.43 \mathrm{E}-24$ $15375.000 \quad------5.77 \mathrm{E}-24 \quad 2.41 \mathrm{E}-24$ $15875.000 \quad-----5.41 \mathrm{E}-24 \quad 2.41 \mathrm{E}-24$ 16375.000 ------- 4.43E-24 2.24E-24 17125.000 ------- $4.19 \mathrm{E}-24 \quad 1.81 \mathrm{E}-24$ 18125.000 ------- 3.84E-24 1.84E-24 19125.000 ------ 3.33E-24 1.50E-24 20125.000 ------ 2.99E-24 $1.42 \mathrm{E}-24$ 21125.000 ------- 2.34E-24 $1.24 \mathrm{E}-24$ 22125.000 ------- 9.96E-25 $1.03 \mathrm{E}-24$ 23125.000 -------- ------- $1.11 \mathrm{E}-24$ 24125.000 -------- ------- 8.83E-25 25125.000 ------- ------- 9.37E-25 26125.000 -------- -------- 9.27E-25 27125.000 ------- ------ 7.43E-25 28125.000 ------- ------- 6.79E-25 29125.000 ------- ------ 6.25E-25 30125.000 ------- ------- 7.22E-25 31125.000 ------- ------- 7.11E-25 32125.000 ------- ------- 5.49E-25 33125.000 ------- ------- 6.25E-25 34125.000 -------- ------- 5.49E-25 35125.000 ------- ------- 4.31E-25 36125.000 ------- ------- 5.60E-25 37875.000 ------- --- 3.88E-25 40375.000 ------- ------- 3.32E-25 42875.000 ------ ------ 3.02E-25 45375.000 ------- ------- 2.41E-25 47875.000 ------- ------- 2.54E-25 50375.000 ------- ------- $1.72 \mathrm{E}-25$ 52875.000 ------- ------- 2.03E-25 55375.000
$2.66 \mathrm{E}-22 \quad 1.23 \mathrm{E}-22 \quad 7.49 \mathrm{E}-23$ $2.28 \mathrm{E}-22 \quad 1.15 \mathrm{E}-22 \quad 6.40 \mathrm{E}-23$ $2.03 \mathrm{E}-22 \quad 9.85 \mathrm{E}-23 \quad 5.73 \mathrm{E}-23$ $1.84 \mathrm{E}-22 \quad 8.44 \mathrm{E}-23 \quad 4.89 \mathrm{E}-23$ $1.64 \mathrm{E}-22 \quad 7.80 \mathrm{E}-23 \quad 4.42 \mathrm{E}-23$ $\begin{array}{lll}1.45 \mathrm{E}-22 & 7.01 \mathrm{E}-23 & 3.55 \mathrm{E}-23\end{array}$ $\begin{array}{lll}1.31 \mathrm{E}-22 & 6.00 \mathrm{E}-23 & 3.48 \mathrm{E}-23\end{array}$ $\begin{array}{lll}1.33 \mathrm{E}-22 & 5.71 \mathrm{E}-23 & 3.26 \mathrm{E}-23\end{array}$ $1.08 \mathrm{E}-22 \quad 5.14 \mathrm{E}-23 \quad 2.80 \mathrm{E}-23$ $9.68 \mathrm{E}-23 \quad 4.60 \mathrm{E}-23 \quad 2.65 \mathrm{E}-23$ 9. 45E-23 4.53E-23 2.54E-23 8.17E-23 $3.91 \mathrm{E}-23 \quad 2.24 \mathrm{E}-23$ $\begin{array}{lll}7.74 \mathrm{E}-23 & 3.72 \mathrm{E}-23 & 2.31 \mathrm{E}-23\end{array}$ $\begin{array}{llll}7.45 \mathrm{E}-23 & 3.56 \mathrm{E}-23 & 2.01 \mathrm{E}-23\end{array}$ $6.73 \mathrm{E}-23 \quad 3.32 \mathrm{E}-23 \quad 1.93 \mathrm{E}-23$ $6.61 \mathrm{E}-23 \quad 2.91 \mathrm{E}-23 \quad 1.62 \mathrm{E}-23$ 6. 28E-23 $2.70 \mathrm{E}-23 \quad 1.56 \mathrm{E}-23$ $\begin{array}{lll}4.71 \mathrm{E}-23 & 2.26 \mathrm{E}-23 & 1.38 \mathrm{E}-23\end{array}$ $\begin{array}{lll}4.96 \mathrm{E}-23 & 2.56 \mathrm{E}-23 & 1.40 \mathrm{E}-23\end{array}$ 5. 23E-23 $2.65 \mathrm{E}-23 \quad 1.19 \mathrm{E}-23$ $4.95 \mathrm{E}-23 \quad 2.00 \mathrm{E}-23 \quad 1.15 \mathrm{E}-23$ 4.06E-23 $1.87 \mathrm{E}-23 \quad 1.01 \mathrm{E}-23$ 3. 20E-23 $1.58 \mathrm{E}-23 \quad 9.65 \mathrm{E}-24$ 3. 15E-23 $1.44 \mathrm{E}-23 \quad 7.60 \mathrm{E}-24$ $2.76 \mathrm{E}-23 \quad 1.22 \mathrm{E}-23 \quad 7.54 \mathrm{E}-24$ $2.39 \mathrm{E}-23 \quad 1.11 \mathrm{E}-23 \quad 6.11 \mathrm{E}-24$ 2. $22 \mathrm{E}-23 \quad 1.09 \mathrm{E}-23 \quad 5.80 \mathrm{E}-24$ $\begin{array}{lll}1.77 \mathrm{E}-23 & 9.23 \mathrm{E}-24 & 5.40 \mathrm{E}-24\end{array}$ $6.18 \mathrm{E}-24 \quad 7.89 \mathrm{E}-24 \quad 4.39 \mathrm{E}-24$ 1.73E-24 8.29E-24 4.67E-24 ------ $6.06 \mathrm{E}-24 \quad 3.88 \mathrm{E}-24$ ------ 7.56E-24 3.29E-24 ------- 5.65E-24 3.97E-24 ------- 5.45E-24 3.29E-24 ------- 4.72E-24 3.07E-24 ------ 5.16E-24 3.10E-24 ------ $4.76 \mathrm{E}-24 \quad 2.08 \mathrm{E}-24$ ----- 3.37E-24 2.62E-24 ------- 3.90E-24 2.59E-24 ------- 4.07E-24 2.34E-24 ------- 3.21E-24 1.86E-24 ------- 3.01E-24 1.52E-24 ------ 2.15E-24 1.53E-24 ------ 2.30E-24 1.32E-24 ------- 1.93E-24 1.34E-24 ------- 5.29E-25 7.74E-25 ------- ------- $1.08 \mathrm{E}-24$ -------- -------- 9.99E-25 ------- ------- 8.58E-25 ------- ------- 8.30E-25 ------- ------- 5.91E-25 ------- ------- 8.16E-25 -c--- ----- 6.75E-25 ------ ------ 5.77E-25 ------ ------ 6.19E-25 ------- ------ 5.21E-25 -------- -------- $4.78 \mathrm{E}-25$ ------- ------- 3.80E-25 ------- ------- 3.66E-25 ------- ------ $4.64 \mathrm{E}-25$ ------- ------- 3.43E-25 ------- ------- 3.83E-25 ------- ------- 3.10E-25 ------ ------ 2.81E-25 ------ ------ 2.87E-25 ------- ------- $1.80 \mathrm{E}-25$ ------- ------ 2.25E-25 ------ ----- 3.94E-26
2.52E-22 $1.50 \mathrm{E}-22 \quad 7.09 \mathrm{E}-23$ $2.16 \mathrm{E}-22 \quad 1.40 \mathrm{E}-22 \quad 6.21 \mathrm{E}-23$ 1.98E-22 $1.17 \mathrm{E}-22 \quad 5.41 \mathrm{E}-23$ $1.63 \mathrm{E}-22 \quad 1.00 \mathrm{E}-22 \quad 4.88 \mathrm{E}-23$ 1.49E-22 9.40E-23 4.32E-23 $\begin{array}{lll}1.30 \mathrm{E}-22 & 8.33 \mathrm{E}-23 & 3.76 \mathrm{E}-23\end{array}$ 1. 20E-22 $7.39 \mathrm{E}-23 \quad 3.48 \mathrm{E}-23$ 1.11E-22 $7.18 \mathrm{E}-23 \quad 3.20 \mathrm{E}-23$

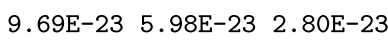
8.73E-23 $5.11 \mathrm{E}-23 \quad 2.53 \mathrm{E}-23$ \begin{tabular}{l}
$8.19 \mathrm{E}-23 \quad 5.11 \mathrm{E}-23 \quad 2.29 \mathrm{E}-23$ \\
\hline $.82 \mathrm{E}-23$
\end{tabular} $\begin{array}{llll}7.82 \mathrm{E}-23 & 4.66 \mathrm{E}-23 & 2.52 \mathrm{E}-23\end{array}$ $6.98 \mathrm{E}-23 \quad 4.16 \mathrm{E}-23 \quad 1.84 \mathrm{E}-23$ 6. $21 \mathrm{E}-23 \quad 4.24 \mathrm{E}-23 \quad 1.82 \mathrm{E}-23$ $6.04 \mathrm{E}-23 \quad 3.77 \mathrm{E}-23 \quad 1.82 \mathrm{E}-23$ $5.60 \mathrm{E}-23 \quad 3.27 \mathrm{E}-23 \quad 1.77 \mathrm{E}-23$ $6.11 \mathrm{E}-23 \quad 3.09 \mathrm{E}-23 \quad 1.46 \mathrm{E}-23$ $4.19 \mathrm{E}-23 \quad 3.08 \mathrm{E}-23 \quad 1.44 \mathrm{E}-23$ $\begin{array}{lll}4.36 \mathrm{E}-23 & 2.85 \mathrm{E}-23 & 1.21 \mathrm{E}-23\end{array}$ $4.41 \mathrm{E}-23 \quad 2.60 \mathrm{E}-23 \quad 1.07 \mathrm{E}-23$ 4.15E-23 2.51E-23 1.14E-23 3. $37 \mathrm{E}-23 \quad 2.12 \mathrm{E}-23 \quad 1.08 \mathrm{E}-23$ 3. $07 \mathrm{E}-23 \quad 1.86 \mathrm{E}-23 \quad 8.92 \mathrm{E}-24$ $2.78 \mathrm{E}-23 \quad 1.69 \mathrm{E}-23 \quad 7.83 \mathrm{E}-24$ $2.13 \mathrm{E}-23 \quad 1.40 \mathrm{E}-23 \quad 7.72 \mathrm{E}-24$ $2.19 \mathrm{E}-23 \quad 1.30 \mathrm{E}-23 \quad 6.15 \mathrm{E}-24$ $1.61 \mathrm{E}-23 \quad 1.11 \mathrm{E}-23 \quad 5.72 \mathrm{E}-24$ $1.50 \mathrm{E}-23 \quad 1.00 \mathrm{E}-23 \quad 5.64 \mathrm{E}-24$ 8. $48 \mathrm{E}-24 \quad 9.13 \mathrm{E}-24 \quad 4.41 \mathrm{E}-24$ $1.58 \mathrm{E}-24 \quad 9.77 \mathrm{E}-24 \quad 3.86 \mathrm{E}-24$ ------ $8.71 \mathrm{E}-24 \quad 3.71 \mathrm{E}-24$ ----- 6.81E-24 3.90E-24 ------ 6.81E-24 3.28E-24 ------ 6.44E-24 2.69E-24 $\begin{array}{ll}------6.60 \mathrm{E}-24 & 2.22 \mathrm{E}-24\end{array}$ ------ 5.33E-24 2.37E-24 ------ 4.59E-24 3.17E-24 ------ 4.80E-24 2.33E-24 ------ $3.75 \mathrm{E}-24 \quad 2.26 \mathrm{E}-24$ ------- 3.80E-24 $1.86 \mathrm{E}-24$ ------- 4.07E-24 1.78E-24 ------- 3.17E-24 1.33E-24 ------- 2.82E-24 1.62E-24 ------ 2.72E-24 1.22E-24 ------- 2.11E-24 1.37E-24 ------ 4.75E-25 1.33E-24 ------- $1.58 \mathrm{E}-25 \quad 1.18 \mathrm{E}-24$ ------- ------ 6.92E-25 -------- ------- 7.65E-25 -------- ------- 5.64E-25 ------- ------ 7.10E-25 ------ ------ 6.74E-25 ------- ------ $8.38 \mathrm{E}-25$ ------- ------- 6.01E-25 ------- ------- 4.01E-25 ------- ------ 4.73E-25 -------- -------- 3.82E-25 ------- ------- 4.19E-25 ------ ------ 7.47E-25 ------ ------ 5.28E-25 ------- ------- 3.57E-25 ------- ------- 2.99E-25 ------ ------- 2.40E-25 ------- ------- 2.55E-25 ------- ------- 2.91E-25 ------- ------- 2.11E-25 ------- ------ $1.09 \mathrm{E}-25$ 
Table 15

The singly differential cross section as a function of the ejected electron angle for single ionization in $5000-25000 \mathrm{keV} / \mathrm{u} \mathrm{O}^{0}, 0^{\wedge}+, 0^{\wedge} 2++$ H_2 collisions.

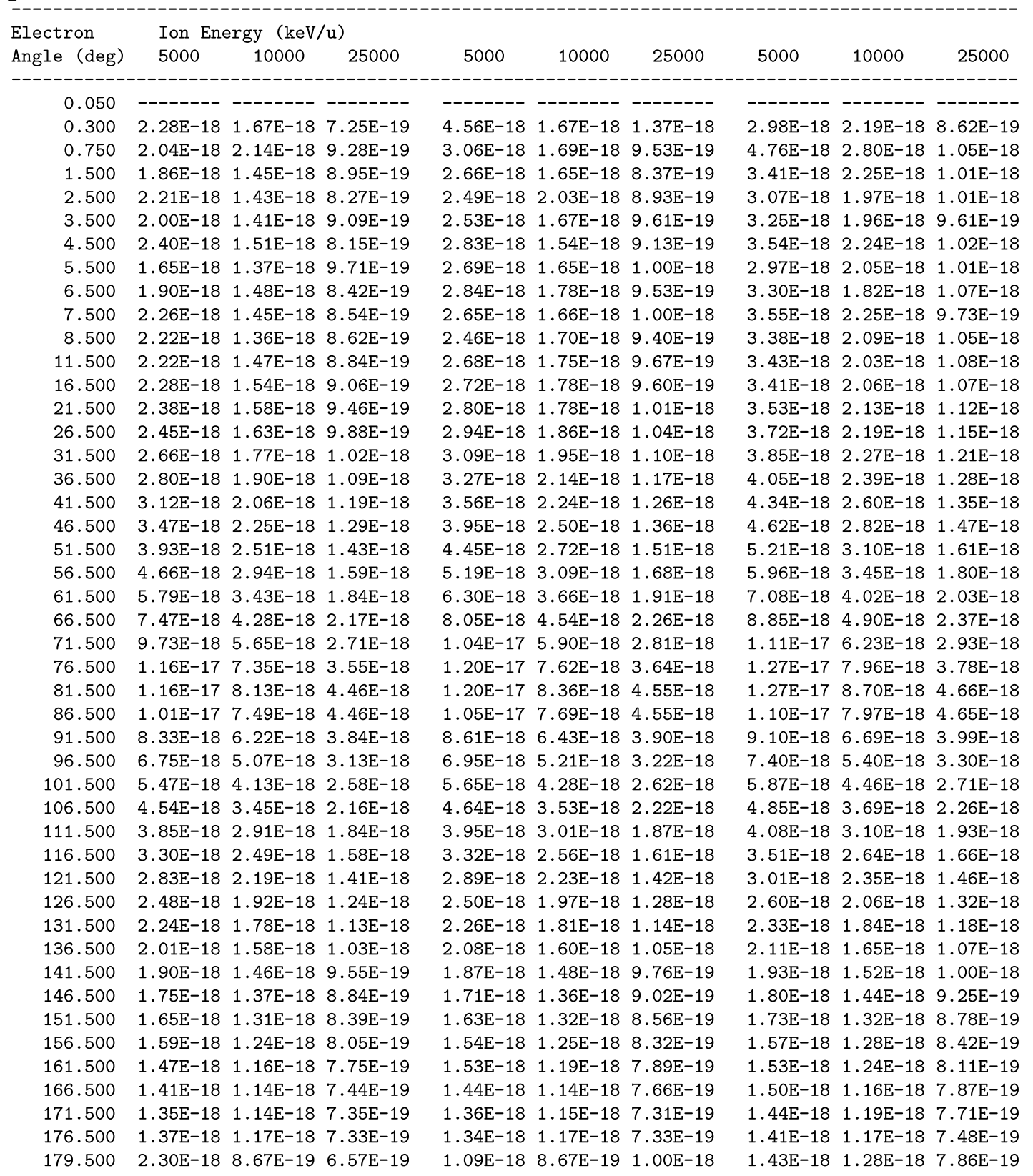


Table 16

The singly differential cross section as a function of the ejected electron angle for single ionization in $5000-25000 \mathrm{keV} / \mathrm{u} \mathrm{O`3+,} 0^{\wedge} 4+, \mathrm{O}^{\wedge} 5+$ + H_2 collisions.

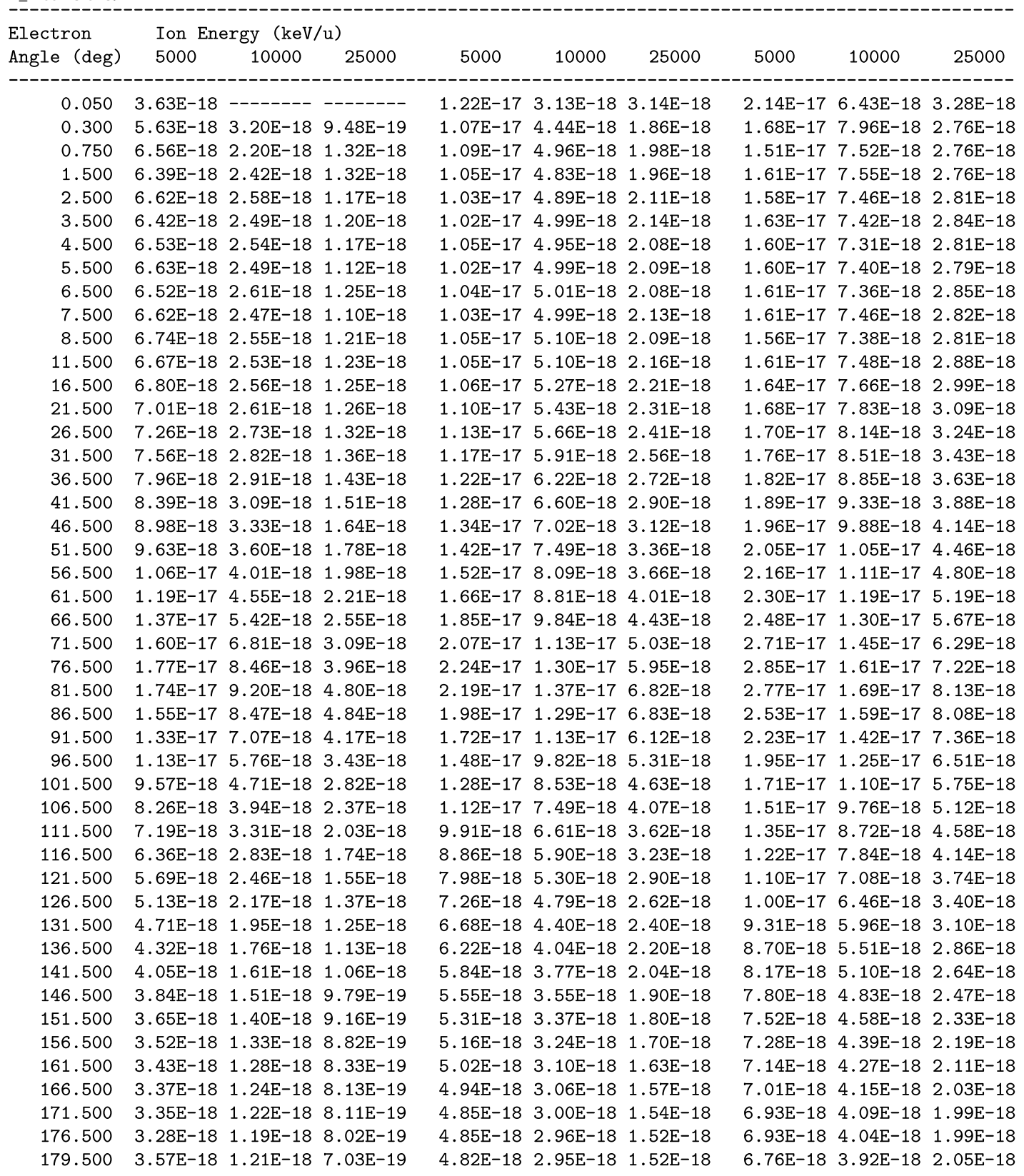


Table 17

The singly differential cross section as a function of the ejected electron angle for single ionization in $5000-25000 \mathrm{keV} / \mathrm{u} 0^{\wedge} 6+, 0^{\wedge} 7^{+}, 0^{\wedge} 8^{+}$ $+\mathrm{H} \_2$ collisions.

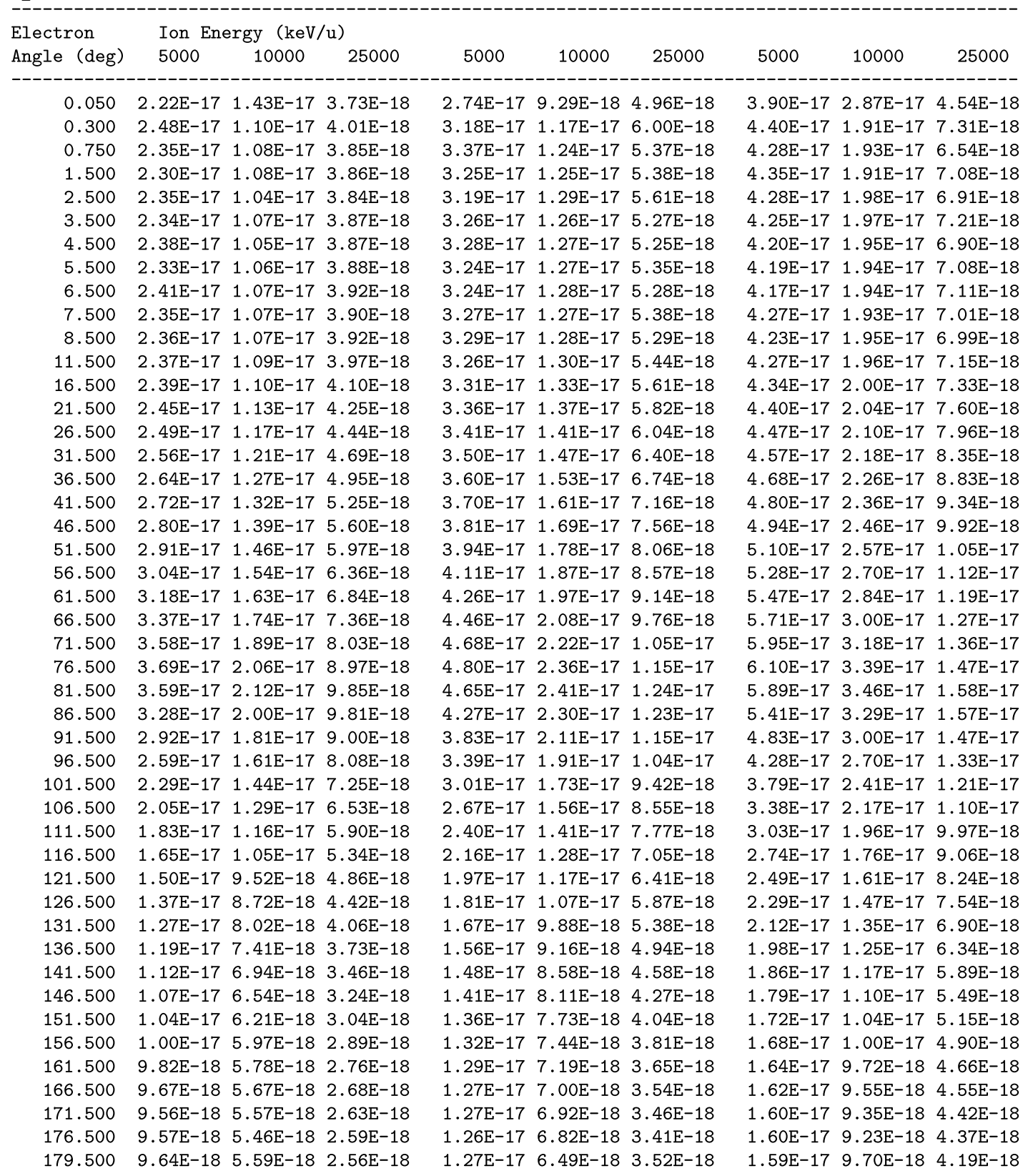


Table 18

The singly differential cross section as a function of the ejected electron energy for double ionization in 5000-25000 keV/u 0, $0^{\wedge}+, 0^{\wedge} 2+$ $+\mathrm{H} \_2$ collisions.

\begin{tabular}{|c|c|c|c|c|c|c|c|c|c|}
\hline \multirow{2}{*}{$\begin{array}{l}\text { Electron } \\
\text { Energy (eV) }\end{array}$} & \multicolumn{3}{|c|}{ Ion Energy $(\mathrm{keV} / \mathrm{u})$} & \multirow[b]{2}{*}{5000} & \multirow[b]{2}{*}{10000} & \multirow[b]{2}{*}{25000} & \multirow[b]{2}{*}{5000} & \multirow[b]{2}{*}{10000} & \multirow[b]{2}{*}{25000} \\
\hline & 5000 & 10000 & 25000 & & & & & & \\
\hline 0.005 & $9.95 \mathrm{E}-20$ & $2.87 \mathrm{E}-20$ & $1.48 \mathrm{E}-20$ & $1.10 \mathrm{E}-19$ & $1 \mathrm{E}-20$ & $2 E-20$ & $8.55 \mathrm{E}-20$ & $5.03 E-20$ & $2.47 \mathrm{E}-20$ \\
\hline 0.030 & $1.22 \mathrm{E}-19$ & $7.02 \mathrm{E}-20$ & $2.31 \mathrm{E}-20$ & $1.47 \mathrm{E}-19$ & $5.51 \mathrm{E}-20$ & $2.22 \mathrm{E}-20$ & $1.95 \mathrm{E}-19$ & $8.35 E-20$ & $2.65 \mathrm{E}-20$ \\
\hline 0.075 & 2. $20 \mathrm{E}-19$ & $1.10 \mathrm{E}-19$ & $3.86 \mathrm{E}-20$ & $2.50 \mathrm{E}-19$ & 1. $12 \mathrm{E}-19$ & $3.88 \mathrm{E}-20$ & $3.03 E-19$ & $1.47 \mathrm{E}-19$ & $4.02 \mathrm{E}-20$ \\
\hline 0.150 & $2.24 \mathrm{E}-19$ & $9.96 \mathrm{E}-20$ & $4.11 \mathrm{E}-20$ & $2.34 \mathrm{E}-19$ & $1.11 \mathrm{E}-19$ & $4.61 \mathrm{E}-20$ & $2.98 \mathrm{E}-19$ & $1.36 \mathrm{E}-19$ & $4.87 \mathrm{E}-20$ \\
\hline 0.350 & $1.90 \mathrm{E}-19$ & $1.01 \mathrm{E}-19$ & $3.86 \mathrm{E}-20$ & $2.17 \mathrm{E}-19$ & $1.16 \mathrm{E}-19$ & $4.34 \mathrm{E}-20$ & $2.69 \mathrm{E}-19$ & $1.35 \mathrm{E}-19$ & $4.96 \mathrm{E}-20$ \\
\hline 0.600 & 1.8 & $9.67 \mathrm{E}-20$ & $4.10 \mathrm{E}-20$ & $2.28 \mathrm{E}-19$ & $1.17 \mathrm{E}-19$ & $4.36 \mathrm{E}-20$ & $2.78 \mathrm{E}-19$ & $1.23 \mathrm{E}-19$ & $4.70 \mathrm{E}-20$ \\
\hline 0.850 & 1.82 & $9.02 \mathrm{E}-20$ & $3.69 \mathrm{E}-20$ & $2.07 \mathrm{E}-19$ & $1.08 \mathrm{E}-19$ & $4.23 \mathrm{E}-20$ & $2.48 \mathrm{E}-19$ & $1.31 \mathrm{E}-19$ & $4.44 \mathrm{E}-20$ \\
\hline 1.500 & $1.65 \mathrm{E}-19$ & $9.16 \mathrm{E}-20$ & $3.49 \mathrm{E}-20$ & $1.89 \mathrm{E}-19$ & $1.04 \mathrm{E}-19$ & $3.89 \mathrm{E}-20$ & $2.39 \mathrm{E}-19$ & $1.18 \mathrm{E}-19$ & $4.40 \mathrm{E}-20$ \\
\hline 3.500 & $1.43 \mathrm{E}-19$ & $7.63 \mathrm{E}-20$ & $2.89 \mathrm{E}-20$ & $1.67 \mathrm{E}-19$ & $8.47 \mathrm{E}-20$ & $3.13 \mathrm{E}-20$ & $2.03 \mathrm{E}-19$ & $9.94 \mathrm{E}-20$ & $3.47 \mathrm{E}-20$ \\
\hline 6.000 & $1.17 \mathrm{E}-19$ & $6.31 \mathrm{E}-20$ & $2.33 \mathrm{E}-20$ & $1.38 \mathrm{E}-19$ & $6.84 \mathrm{E}-20$ & $2.49 \mathrm{E}-20$ & $1.69 \mathrm{E}-19$ & $8.00 \mathrm{E}-20$ & $2.72 \mathrm{E}-20$ \\
\hline 8.500 & $1.01 \mathrm{E}-19$ & $5.32 \mathrm{E}-20$ & $1.91 \mathrm{E}-20$ & $1.13 \mathrm{E}-19$ & $5.78 \mathrm{E}-20$ & $2.05 \mathrm{E}-20$ & $1.38 \mathrm{E}-19$ & $6.73 \mathrm{E}-20$ & $2.32 \mathrm{E}-20$ \\
\hline 12.500 & 8.1 & $4.07 \mathrm{E}-20$ & $1.53 \mathrm{E}-20$ & $9.10 \mathrm{E}-20$ & $4.51 \mathrm{E}-20$ & $1.60 \mathrm{E}-20$ & $1.08 \mathrm{E}-19$ & $5.21 \mathrm{E}-20$ & $1.72 \mathrm{E}-20$ \\
\hline 17.500 & 6.3 & $3.14 \mathrm{E}-20$ & $1.14 \mathrm{E}-20$ & $7.01 \mathrm{E}-20$ & $3.31 \mathrm{E}-20$ & $1.19 \mathrm{E}-20$ & $8.17 \mathrm{E}-20$ & $3.70 \mathrm{E}-20$ & $1.28 \mathrm{E}-20$ \\
\hline 22.500 & 4.9 & $2.44 \mathrm{E}-20$ & $8.41 \mathrm{E}-21$ & $5.56 \mathrm{E}-20$ & $2.70 \mathrm{E}-20$ & $9.38 \mathrm{E}-21$ & $6.52 \mathrm{E}-20$ & $3.00 \mathrm{E}-20$ & $1.00 \mathrm{E}-20$ \\
\hline 27.500 & 4.1 & $2.06 \mathrm{E}-20$ & $6.92 \mathrm{E}-21$ & $4.50 \mathrm{E}-20$ & $2.13 \mathrm{E}-20$ & $7.20 \mathrm{E}-21$ & $5.25 \mathrm{E}-20$ & $2.44 \mathrm{E}-20$ & $7.91 \mathrm{E}-21$ \\
\hline 32.500 & 3.4 & $1.72 \mathrm{E}-20$ & $5.96 \mathrm{E}-21$ & $3.80 \mathrm{E}-20$ & $1.86 \mathrm{E}-20$ & $6.09 \mathrm{E}-21$ & $4.29 \mathrm{E}-20$ & $2.04 \mathrm{E}-20$ & $6.34 \mathrm{E}-21$ \\
\hline 37.500 & $2.96 \mathrm{E}-20$ & $1.36 \mathrm{E}-20$ & $4.77 \mathrm{E}-21$ & $3.24 \mathrm{E}-20$ & $1.58 \mathrm{E}-20$ & $5.02 \mathrm{E}-21$ & $3.77 \mathrm{E}-20$ & $1.76 \mathrm{E}-20$ & $5.39 \mathrm{E}-21$ \\
\hline 42.500 & $2.56 \mathrm{E}-20$ & $1.22 \mathrm{E}-20$ & $4.21 \mathrm{E}-21$ & $2.81 \mathrm{E}-20$ & $1.35 \mathrm{E}-20$ & $4.21 \mathrm{E}-21$ & $3.27 \mathrm{E}-20$ & $1.51 \mathrm{E}-20$ & $4.48 \mathrm{E}-21$ \\
\hline 47.500 & $2.26 \mathrm{E}-20$ & $1.05 \mathrm{E}-20$ & $3.40 \mathrm{E}-21$ & $2.43 \mathrm{E}-20$ & $1.15 \mathrm{E}-20$ & $3.54 \mathrm{E}-21$ & $2.86 \mathrm{E}-20$ & $1.23 \mathrm{E}-20$ & $3.85 \mathrm{E}-21$ \\
\hline 52.500 & $1.98 \mathrm{E}-20$ & $9.26 \mathrm{E}-21$ & $3.01 \mathrm{E}-21$ & $2.12 \mathrm{E}-20$ & $9.94 \mathrm{E}-21$ & $3.16 \mathrm{E}-21$ & $2.33 \mathrm{E}-20$ & $1.07 \mathrm{E}-20$ & $3.37 \mathrm{E}-21$ \\
\hline 57.500 & $1.80 \mathrm{E}-20$ & $8.24 \mathrm{E}-21$ & $2.60 \mathrm{E}-21$ & $1.95 \mathrm{E}-20$ & $8.69 \mathrm{E}-21$ & $2.83 \mathrm{E}-21$ & $2.12 \mathrm{E}-20$ & $9.52 \mathrm{E}-21$ & $2.91 \mathrm{E}-21$ \\
\hline 62.500 & 1.5 & $7.04 \mathrm{E}$ & $2.27 \mathrm{E}-21$ & $1.68 \mathrm{E}-20$ & $7.78 \mathrm{E}-21$ & $2.56 \mathrm{E}-21$ & $1.87 \mathrm{E}-20$ & $8.55 \mathrm{E}-21$ & $2.57 \mathrm{E}-21$ \\
\hline 70.000 & 1.3 & $6.05 \mathrm{E}-21$ & $1.91 \mathrm{E}-21$ & $1.47 \mathrm{E}-20$ & $6.60 \mathrm{E}-21$ & $2.04 \mathrm{E}-21$ & $1.68 \mathrm{E}-20$ & $7.12 \mathrm{E}-21$ & $2.15 \mathrm{E}-21$ \\
\hline 80.000 & 1.1 & 4.89 & $1.53 \mathrm{E}-21$ & 1. $22 \mathrm{E}-20$ & $5.44 \mathrm{E}-21$ & $1.65 \mathrm{E}-21$ & $1.40 \mathrm{E}-20$ & $5.92 \mathrm{E}-21$ & $1.80 \mathrm{E}-21$ \\
\hline 90 & 9.5 & 4.3 & $1.40 \mathrm{E}-21$ & $1.06 \mathrm{E}-20$ & $4.28 \mathrm{E}-21$ & $1.40 \mathrm{E}-21$ & $1.15 \mathrm{E}-20$ & $5.09 \mathrm{E}-21$ & $1.53 \mathrm{E}-21$ \\
\hline 100 & 8.5 & 3.7 & $E-21$ & $8.73 \mathrm{E}-21$ & $4.01 \mathrm{E}-21$ & $1.18 \mathrm{E}-21$ & $9.96 \mathrm{E}-21$ & $4.11 \mathrm{E}-21$ & $1.25 \mathrm{E}-21$ \\
\hline & 7. & 3.2 & -22 & $7.79 \mathrm{E}-21$ & 3.2 & 9. & 8. & -21 & $1.03 \mathrm{E}-21$ \\
\hline $12 C$ & 6. & 2.7 & -22 & $6.64 \mathrm{E}-21$ & 3.0 & 9. & -21 & -21 & $8.85 \mathrm{E}-22$ \\
\hline $13 c$ & -21 & 2.3 & $E-22$ & $6.02 \mathrm{E}-21$ & $2.57 \mathrm{E}-21$ & $7.40 \mathrm{E}-22$ & $6.48 \mathrm{E}-21$ & $E-21$ & $7.52 \mathrm{E}-22$ \\
\hline 14 & 5.3 & 2.0 & -22 & $5.34 \mathrm{E}-21$ & 2. $33 \mathrm{E}-21$ & $6.52 \mathrm{E}-22$ & o. $16 \mathrm{E}-21$ & $2.46 \mathrm{E}-21$ & $7.43 \mathrm{E}-22$ \\
\hline 15 & 4.6 & 1.88 & $5.58 \mathrm{E}-22$ & $5.04 \mathrm{E}-21$ & $1.98 \mathrm{E}-21$ & $5.55 \mathrm{E}-22$ & $5.51 \mathrm{E}-21$ & $2.07 \mathrm{E}-21$ & $6.11 \mathrm{E}-22$ \\
\hline 160.000 & 4.1 & 1.90 & $5.08 \mathrm{E}-22$ & $4.25 \mathrm{E}-21$ & $1.84 \mathrm{E}-21$ & $5.42 \mathrm{E}-22$ & -21 & $2 E-21$ & $6.00 \mathrm{E}-22$ \\
\hline 170.000 & 3.5 & $1.59 \mathrm{E}-21$ & $4.58 \mathrm{E}-22$ & $4.18 \mathrm{E}-21$ & $1.53 \mathrm{E}-21$ & $4.60 \mathrm{E}-22$ & $4.79 \mathrm{E}-21$ & $1.75 \mathrm{E}-21$ & $4.86 \mathrm{E}-22$ \\
\hline 187.500 & 3. $32 \mathrm{E}-21$ & $1.31 \mathrm{E}-21$ & $3.79 \mathrm{E}-22$ & $3.55 \mathrm{E}-21$ & $1.44 \mathrm{E}-21$ & $3.90 \mathrm{E}-22$ & $3.86 \mathrm{E}-21$ & $1.50 \mathrm{E}-21$ & $4.04 \mathrm{E}-22$ \\
\hline 212.500 & $2.61 \mathrm{E}-21$ & 1.14 & $3.08 \mathrm{E}-22$ & $2.97 \mathrm{E}-21$ & $1.11 \mathrm{E}-21$ & $3.35 \mathrm{E}-22$ & $3.18 \mathrm{E}-21$ & $1.20 \mathrm{E}-21$ & $3.28 \mathrm{E}-22$ \\
\hline 237 & 2.2 & 9.1 & $2.65 \mathrm{E}-22$ & 21 & $9.20 \mathrm{E}-22$ & $2.63 \mathrm{E}-22$ & $.58 \mathrm{E}-21$ & $1.07 \mathrm{E}-21$ & $2.75 \mathrm{E}-22$ \\
\hline 262.500 & $1.81 \mathrm{E}-21$ & $7.57 \mathrm{E}-22$ & $1.99 \mathrm{E}-22$ & $2.15 \mathrm{E}-21$ & $8.45 \mathrm{E}-22$ & $2.10 \mathrm{E}-22$ & $2.30 \mathrm{E}-21$ & $8.43 \mathrm{E}-22$ & $2.32 \mathrm{E}-22$ \\
\hline 287.500 & $1.58 \mathrm{E}-21$ & $6.41 \mathrm{E}-22$ & $1.75 \mathrm{E}-22$ & $1.82 \mathrm{E}-21$ & $7.29 \mathrm{E}-22$ & $1.78 \mathrm{E}-22$ & $1.93 \mathrm{E}-21$ & $7.28 \mathrm{E}-22$ & $1.89 \mathrm{E}-22$ \\
\hline 312.500 & $1.41 \mathrm{E}-21$ & $5.44 \mathrm{E}-22$ & $1.43 \mathrm{E}-22$ & $1.40 \mathrm{E}-21$ & $5.91 \mathrm{E}-22$ & $1.61 \mathrm{E}-22$ & $1.75 \mathrm{E}-21$ & $6.00 \mathrm{E}-22$ & $1.75 \mathrm{E}-22$ \\
\hline 350.000 & $1.17 \mathrm{E}-21$ & $4.58 \mathrm{E}-22$ & $1.31 \mathrm{E}-22$ & 1. $23 \mathrm{E}-21$ & $4.94 \mathrm{E}-22$ & 1. $32 \mathrm{E}-22$ & $1.35 \mathrm{E}-21$ & $5.04 \mathrm{E}-22$ & 1. $32 \mathrm{E}-22$ \\
\hline 400.000 & $9.96 \mathrm{E}-22$ & 3. $83 \mathrm{E}-22$ & $1.01 \mathrm{E}-22$ & $1.02 \mathrm{E}-21$ & $3.98 \mathrm{E}-22$ & $1.09 \mathrm{E}-22$ & $1.05 \mathrm{E}-21$ & $4.06 \mathrm{E}-22$ & $1.04 \mathrm{E}-22$ \\
\hline 450.000 & $7.54 \mathrm{E}-22$ & 2.87 & $8.51 \mathrm{E}-23$ & $8.39 \mathrm{E}-22$ & $3.18 \mathrm{E}-22$ & $8.32 \mathrm{E}-23$ & $8.83 E-22$ & $3.05 \mathrm{E}-22$ & $8.65 \mathrm{E}-23$ \\
\hline 500.000 & $6.67 \mathrm{E}-22$ & 2.5 & $6.07 \mathrm{E}-23$ & $6.84 \mathrm{E}-22$ & $2.60 \mathrm{E}-22$ & $6.82 \mathrm{E}-23$ & $7.12 \mathrm{E}-22$ & $2.82 \mathrm{E}-22$ & $7.08 \mathrm{E}-23$ \\
\hline & -22 & 2.0 & $5.53 \mathrm{E}-23$ & $5.80 \mathrm{E}-22$ & $2.18 \mathrm{E}-22$ & $5.62 \mathrm{E}-23$ & $9 \mathrm{E}-22$ & $2.17 \mathrm{E}-22$ & $6.06 \mathrm{E}-23$ \\
\hline & 4.8 & 1.6 & $4.20 \mathrm{E}-23$ & $4.88 \mathrm{E}-22$ & $2.09 \mathrm{E}-22$ & $4.84 \mathrm{E}-23$ & DE-22 & $2.08 \mathrm{E}-22$ & $4.89 \mathrm{E}-23$ \\
\hline & & 1.4 & 3.5 & $E-22$ & $E-22$ & -23 & -22 & & $4.17 \mathrm{E}-23$ \\
\hline & & 1. & $3 . c$ & & 1.1 & -23 & -22 & & $2.90 \mathrm{E}-23$ \\
\hline & & 8. & 2 & -22 & 8.8 & -23 & -22 & -22 & $2.46 \mathrm{E}-23$ \\
\hline & -22 & 7.1 & $E-23$ & $2.24 \mathrm{E}-22$ & $7.92 \mathrm{E}-23$ & -23 & 1.95E-22 & $7.72 \mathrm{E}-23$ & $1.92 \mathrm{E}-23$ \\
\hline .000 & $1.63 \mathrm{E}-22$ & 6.2 & $1.51 \mathrm{E}-23$ & $1.67 \mathrm{E}-22$ & $7.28 \mathrm{E}-23$ & $1.86 \mathrm{E}-23$ & $1.91 \mathrm{E}-22$ & $6.68 \mathrm{E}-23$ & $1.65 \mathrm{E}-23$ \\
\hline 00 & 1.5 & 5.1 & -23 & $.36 \mathrm{E}-22$ & 4.69 & $1.38 \mathrm{E}-23$ & $E-22$ & $E-23$ & $1.54 \mathrm{E}-23$ \\
\hline 1275.000 & $E-22$ & 4.5 & $1.04 \mathrm{E}-23$ & 1. $35 \mathrm{E}-22$ & $4.28 \mathrm{E}-23$ & $1.15 \mathrm{E}-23$ & $1.44 \mathrm{E}-22$ & $5.32 \mathrm{E}-23$ & $1.19 \mathrm{E}-23$ \\
\hline 1375.000 & $1.00 \mathrm{E}-22$ & $4.73 \mathrm{E}-23$ & $9.25 \mathrm{E}-24$ & 1.20E-22 & $3.54 \mathrm{E}-23$ & $9.15 \mathrm{E}-24$ & 1. $25 \mathrm{E}-22$ & $4.13 \mathrm{E}-23$ & $9.40 \mathrm{E}-24$ \\
\hline 1475.000 & $1.06 \mathrm{E}-22$ & $3.42 \mathrm{E}-23$ & $7.67 \mathrm{E}-24$ & 9. $42 \mathrm{E}-23$ & $3.51 \mathrm{E}-23$ & $7.95 \mathrm{E}-24$ & $1.12 \mathrm{E}-22$ & $3.81 \mathrm{E}-23$ & $9.40 \mathrm{E}-24$ \\
\hline 1575.000 & $8.79 \mathrm{E}-23$ & $3.19 \mathrm{E}-23$ & $6.93 \mathrm{E}-24$ & 7. $37 \mathrm{E}-23$ & $3.03 E-23$ & $8.14 \mathrm{E}-24$ & $.86 \mathrm{E}-23$ & $2.95 \mathrm{E}-23$ & $6.73 \mathrm{E}-24$ \\
\hline 1675.000 & 7.8 & 2.3 & $6.47 \mathrm{E}-24$ & $.86 \mathrm{E}-23$ & $3.00 \mathrm{E}-23$ & $6.01 \mathrm{E}-24$ & $55 \mathrm{E}-23$ & $2.87 \mathrm{E}-23$ & $6.63 \mathrm{E}-24$ \\
\hline 1775.000 & $7.28 \mathrm{E}-23$ & 2.6 & $6.01 \mathrm{E}-24$ & $.97 \mathrm{E}-23$ & $2.04 \mathrm{E}-23$ & $4.35 \mathrm{E}-24$ & $.78 \mathrm{E}-23$ & $2.80 \mathrm{E}-23$ & $5.94 \mathrm{E}-24$ \\
\hline 1875.000 & $6.75 \mathrm{E}-23$ & $1.88 \mathrm{E}-$ & $5.18 \mathrm{E}-24$ & $6.18 \mathrm{E}-23$ & $2.46 \mathrm{E}-23$ & $5.92 \mathrm{E}-24$ & $.04 \mathrm{E}-23$ & $1.90 \mathrm{E}-23$ & $5.54 \mathrm{E}-24$ \\
\hline 1975.000 & $5.02 \mathrm{E}-23$ & $1.69 \mathrm{E}-23$ & $5.82 \mathrm{E}-24$ & $5.50 \mathrm{E}-23$ & $1.82 \mathrm{E}-23$ & $4.53 \mathrm{E}-24$ & $6.67 \mathrm{E}-23$ & $1.98 \mathrm{E}-23$ & $4.55 \mathrm{E}-24$ \\
\hline 2075.000 & $4.34 \mathrm{E}-23$ & $1.85 \mathrm{E}-23$ & $3.70 \mathrm{E}-24$ & $5.65 \mathrm{E}-23$ & $1.66 \mathrm{E}-23$ & $5.18 \mathrm{E}-24$ & $5.70 \mathrm{E}-23$ & $1.87 \mathrm{E}-23$ & $3.66 \mathrm{E}-24$ \\
\hline 2175.000 & $4.50 \mathrm{E}-23$ & $1.66 \mathrm{E}-23$ & $3.79 \mathrm{E}-24$ & $5.39 \mathrm{E}-23$ & $1.66 \mathrm{E}-23$ & $3.33 \mathrm{E}-24$ & $5.53 \mathrm{E}-23$ & $2.12 \mathrm{E}-23$ & $4.25 \mathrm{E}-24$ \\
\hline 2275.000 & $4.19 \mathrm{E}-23$ & $1.37 \mathrm{E}-23$ & $2.50 \mathrm{E}-24$ & $4.97 \mathrm{E}-23$ & $1.21 \mathrm{E}-23$ & $3.24 \mathrm{E}-24$ & $4.96 \mathrm{E}-23$ & $1.44 \mathrm{E}-23$ & $3.96 \mathrm{E}-24$ \\
\hline 2375.000 & $3.51 \mathrm{E}-23$ & $1.47 \mathrm{E}-23$ & $3.14 \mathrm{E}-24$ & $4.29 \mathrm{E}-23$ & $1.37 \mathrm{E}-23$ & $3.24 \mathrm{E}-24$ & $4.27 \mathrm{E}-23$ & $1.54 \mathrm{E}-23$ & $3.96 \mathrm{E}-24$ \\
\hline 2475.000 & $4.08 \mathrm{E}-23$ & $1.56 \mathrm{E}-23$ & $2.68 \mathrm{E}-24$ & $3.87 \mathrm{E}-23$ & $1.56 \mathrm{E}-23$ & $2.96 \mathrm{E}-24$ & $4.27 \mathrm{E}-23$ & $1.65 \mathrm{E}-23$ & $3.17 \mathrm{E}-24$ \\
\hline 2575.000 & $3.77 \mathrm{E}-23$ & $1.31 \mathrm{E}-23$ & $2.77 \mathrm{E}-24$ & $3.04 E-23$ & $1.60 \mathrm{E}-23$ & $2.68 \mathrm{E}-24$ & $4.45 \mathrm{E}-23$ & $1.90 \mathrm{E}-23$ & $2.67 \mathrm{E}-24$ \\
\hline
\end{tabular}


Table 18 (continued)

$\begin{array}{lllll}2725.000 & 2.88 \mathrm{E}-23 & 9.90 \mathrm{E}-24 & 2.27 \mathrm{E}-24\end{array}$ $2925.000 \quad 2.85 \mathrm{E}-23 \quad 7.34 \mathrm{E}-24 \quad 2.36 \mathrm{E}-24$

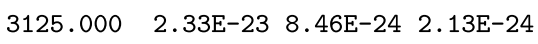
$3325.000 \quad 2.33 \mathrm{E}-23 \quad 7.34 \mathrm{E}-24 \quad 2.17 \mathrm{E}-24$ $3525.000 \quad 1.81 \mathrm{E}-23 \quad 7.34 \mathrm{E}-24 \quad 1.80 \mathrm{E}-24$ $3725.000 \quad 1.94 \mathrm{E}-23 \quad 7.34 \mathrm{E}-24 \quad 1.53 \mathrm{E}-24$ $3925.000 \quad 1.47 \mathrm{E}-23 \quad 6.39 \mathrm{E}-24 \quad 1.53 \mathrm{E}-24$ $4125.000 \quad 1.52 \mathrm{E}-23 \quad 4.79 \mathrm{E}-24 \quad 1.43 \mathrm{E}-24$ $4325.000 \quad 1.49 \mathrm{E}-23 \quad 3.67 \mathrm{E}-24 \quad 7.40 \mathrm{E}-25$ $4525.000 \quad 1.07 \mathrm{E}-23 \quad 5.11 \mathrm{E}-24 \quad 9.71 \mathrm{E}-25$ $4725.000 \quad 1.15 \mathrm{E}-23 \quad 3.19 \mathrm{E}-24 \quad 1.02 \mathrm{E}-24$ $4925.000 \quad 1.05 \mathrm{E}-23 \quad 4.63 \mathrm{E}-24 \quad 1.16 \mathrm{E}-24$ $5125.000 \quad 1.10 \mathrm{E}-23 \quad 2.71 \mathrm{E}-24 \quad 9.25 \mathrm{E}-25$ $5325.000 \quad 9.42 \mathrm{E}-24 \quad 2.55 \mathrm{E}-24 \quad 7.86 \mathrm{E}-25$ $5525.000 \quad 5.50 \mathrm{E}-24 \quad 1.76 \mathrm{E}-24 \quad 3.24 \mathrm{E}-25$ $5725.000 \quad 6.80 \mathrm{E}-24 \quad 2.39 \mathrm{E}-24 \quad 5.55 \mathrm{E}-25$


$6125.000 \quad 5.76 \mathrm{E}-24 \quad 2.71 \mathrm{E}-24 \quad 4.62 \mathrm{E}-25$ $6325.000 \quad 7.33 \mathrm{E}-24 \quad 2.08 \mathrm{E}-24 \quad 5.55 \mathrm{E}-25$ $6525.000 \quad 6.54 \mathrm{E}-24 \quad 2.08 \mathrm{E}-24 \quad 4.16 \mathrm{E}-25$ $6875.000 \quad 4.71 \mathrm{E}-24 \quad 2.49 \mathrm{E}-24 \quad 3.70 \mathrm{E}-25$ $7375.000 \quad 3.45 \mathrm{E}-24 \quad 1.40 \mathrm{E}-24 \quad 2.96 \mathrm{E}-25$ $\begin{array}{llll}7875.000 & 4.29 \mathrm{E}-24 & 1.66 \mathrm{E}-24 & 2.40 \mathrm{E}-25\end{array}$ $8375.000 \quad 4.61 \mathrm{E}-24 \quad 1.79 \mathrm{E}-24 \quad 3.88 \mathrm{E}-25$ $8875.000 \quad 3.14 \mathrm{E}-24 \quad 1.34 \mathrm{E}-24 \quad 2.03 \mathrm{E}-25$ $\begin{array}{llll}9375.000 & 3.45 \mathrm{E}-24 & 9.58 \mathrm{E}-25 & 1.29 \mathrm{E}-25\end{array}$ $9875.000 \quad 2.20 \mathrm{E}-24 \quad 7.02 \mathrm{E}-25 \quad 1.11 \mathrm{E}-25$ $10375.000 \quad 2.41 \mathrm{E}-24 \quad 1.02 \mathrm{E}-24 \quad 1.66 \mathrm{E}-25$ $10875.000 \quad 7.33 \mathrm{E}-25 \quad 4.47 \mathrm{E}-25 \quad 1.85 \mathrm{E}-25$ 11375.000 ------ $5.11 \mathrm{E}-25$------11875.000 ------ 7.02E-25 $1.29 \mathrm{E}-25$ 12375.000 ------ 7.02E-25 $1.29 \mathrm{E}-25$ 12875.000 ------- 3.83E-25 1.85E-25 13375.000 ------ $6.39 \mathrm{E}-25 \quad 1.48 \mathrm{E}-25$ 13875.000 ------ 3.19E-25 $1.48 \mathrm{E}-25$ 14375.000 ------ 3.19E-25 ------14875.000 15375.000 15875.000 16375.000 17125.000 18125.000 19125.000 20125.000 21125.000 22125.000 23125.000 24125.000 25125.000 26125.000 ------- ------ 5.55E-26 -
3. $43 \mathrm{E}-23 \quad 1.29 \mathrm{E}-23 \quad 2.91 \mathrm{E}-24$ $2.64 \mathrm{E}-23 \quad 1.15 \mathrm{E}-23 \quad 2.54 \mathrm{E}-24$ $\begin{array}{lll}1.99 \mathrm{E}-23 & 8.78 \mathrm{E}-24 & 1.66 \mathrm{E}-24\end{array}$ $2.62 \mathrm{E}-23 \quad 6.55 \mathrm{E}-24 \quad 1.94 \mathrm{E}-24$ $2.20 \mathrm{E}-23 \quad 9.58 \mathrm{E}-24 \quad 1.39 \mathrm{E}-24$ $2.15 \mathrm{E}-23 \quad 5.11 \mathrm{E}-24 \quad 1.16 \mathrm{E}-24$ $\begin{array}{lll}1.86 \mathrm{E}-23 & 4.47 \mathrm{E}-24 & 1.29 \mathrm{E}-24\end{array}$ $1.52 \mathrm{E}-23 \quad 3.83 \mathrm{E}-24 \quad 1.39 \mathrm{E}-24$ $1.70 \mathrm{E}-23 \quad 3.51 \mathrm{E}-24 \quad 6.93 \mathrm{E}-25$ 1. $10 \mathrm{E}-23 \quad 3.51 \mathrm{E}-24 \quad 9.25 \mathrm{E}-25$ $9.42 \mathrm{E}-24 \quad 3.03 \mathrm{E}-24 \quad 1.11 \mathrm{E}-24$ 1.05E-23 3.83E-24 4.62E-25 $9.16 \mathrm{E}-24 \quad 4.47 \mathrm{E}-24 \quad 6.01 \mathrm{E}-25$ 5.50E-24 2.55E-24 8.32E-25 $7.33 \mathrm{E}-24 \quad 2.71 \mathrm{E}-24 \quad 5.09 \mathrm{E}-25$ $7.85 \mathrm{E}-24 \quad 2.23 \mathrm{E}-24 \quad 3.70 \mathrm{E}-25$ $9.68 \mathrm{E}-24 \quad 2.55 \mathrm{E}-24 \quad 6.01 \mathrm{E}-25$ 8.11E-24 2.23E-24 2.31E-25 $7.59 \mathrm{E}-24 \quad 2.08 \mathrm{E}-24 \quad 5.09 \mathrm{E}-25$ $4.45 \mathrm{E}-24 \quad 1.92 \mathrm{E}-24 \quad 2.77 \mathrm{E}-25$ $5.13 \mathrm{E}-24 \quad 1.98 \mathrm{E}-24 \quad 4.44 \mathrm{E}-25$ 3. 87E-24 $1.09 \mathrm{E}-24 \quad 3.51 \mathrm{E}-25$ 3. $98 \mathrm{E}-24 \quad 1.40 \mathrm{E}-24 \quad 2.40 \mathrm{E}-25$ 3. 35E-24 $8.94 \mathrm{E}-25 \quad 1.66 \mathrm{E}-25$ $5.55 \mathrm{E}-24 \quad 1.21 \mathrm{E}-24 \quad 2.77 \mathrm{E}-25$ $2.93 \mathrm{E}-24 \quad 1.47 \mathrm{E}-24 \quad 1.11 \mathrm{E}-25$ $3.66 \mathrm{E}-24 \quad 9.58 \mathrm{E}-25 \quad 1.85 \mathrm{E}-25$ $1.99 \mathrm{E}-24 \quad 4.47 \mathrm{E}-25 \quad 1.48 \mathrm{E}-25$ $1.15 \mathrm{E}-24$------ $2.03 \mathrm{E}-25$ ------- $4.47 \mathrm{E}-25 \quad 1.85 \mathrm{E}-25$ ------- 5.11E-25 $1.48 \mathrm{E}-25$ ------- 5.75E-25 9.25E-26 ------- 3.83E-25 -------------- 3.83E-25 -------------- 4.47E-25 9.25E-26 ------- 3.83E-25 --------

------ 7.02E-25 1.29E-25 ------ $3.19 \mathrm{E}-25 \quad 1.29 \mathrm{E}-25$ -------- -------- $1.48 \mathrm{E}-25$ ------- $1.92 \mathrm{E}-25 \quad 4.62 \mathrm{E}-26$ ------- 3.19E-25 8.32E-26 ------- $1.60 \mathrm{E}-25 \quad 5.55 \mathrm{E}-26$

------- $1.92 \mathrm{E}-25$-------------- $2.87 \mathrm{E}-25$-------------- ------- $4.62 \mathrm{E}-26$ ------- ------- $4.62 \mathrm{E}-26$ $\begin{array}{lll}3.79 \mathrm{E}-23 & 1.01 \mathrm{E}-23 & 2.67 \mathrm{E}-24\end{array}$ $2.79 \mathrm{E}-23 \quad 1.17 \mathrm{E}-23 \quad 2.18 \mathrm{E}-24$ $2.05 \mathrm{E}-23 \quad 9.52 \mathrm{E}-24 \quad 2.08 \mathrm{E}-24$ 2. $11 \mathrm{E}-23 \quad 9.34 \mathrm{E}-24 \quad 1.78 \mathrm{E}-24$ $2.17 \mathrm{E}-23 \quad 8.08 \mathrm{E}-24 \quad 1.58 \mathrm{E}-24$ $1.65 \mathrm{E}-23 \quad 8.08 \mathrm{E}-24 \quad 1.78 \mathrm{E}-24$

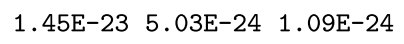
1. 34E-23 4.67E-24 1.39E-24 $1.62 \mathrm{E}-23 \quad 4.31 \mathrm{E}-24 \quad 8.41 \mathrm{E}-25$ 1.51E-23 3.05E-24 6.43E-25 $\begin{array}{lll}1.05 \mathrm{E}-23 & 4.49 \mathrm{E}-24 \quad 5.44 \mathrm{E}-25\end{array}$ 9.97E-24 4.31E-24 6.93E-25 $9.12 \mathrm{E}-24 \quad 3.41 \mathrm{E}-24 \quad 5.94 \mathrm{E}-25$ $9.40 \mathrm{E}-24 \quad 3.05 \mathrm{E}-24 \quad 5.94 \mathrm{E}-25$ $7.12 \mathrm{E}-24$
$3.05 \mathrm{E}-24$ 9. $97 \mathrm{E}-24 \quad 2.87 \mathrm{E}-24 \quad 3.96 \mathrm{E}-25$ $6.55 \mathrm{E}-24 \quad 1.98 \mathrm{E}-24 \quad 8.41 \mathrm{E}-25$ 6.55E-24 1.62E-24 9.89E-25 $4.84 \mathrm{E}-24 \quad 1.44 \mathrm{E}-24 \quad 2.47 \mathrm{E}-25$ 5. 13E-24 2.51E-24 4.95E-25 $5.93 \mathrm{E}-24 \quad 1.51 \mathrm{E}-24 \quad 4.95 \mathrm{E}-25$ $3.76 \mathrm{E}-24 \quad 1.22 \mathrm{E}-24 \quad 4.95 \mathrm{E}-25$ $5.02 \mathrm{E}-24 \quad 1.29 \mathrm{E}-24 \quad 3.36 \mathrm{E}-25$ 4.10E-24 1.80E-24 1.98E-25 3.76E-24 1.08E-24 1.58E-25 $3.53 \mathrm{E}-24 \quad 1.01 \mathrm{E}-24 \quad 2.18 \mathrm{E}-25$ 4.10E-24 8.62E-25 1.78E-25 $2.28 \mathrm{E}-24 \quad 6.46 \mathrm{E}-25 \quad 1.78 \mathrm{E}-25$ 1.14E-24 7.18E-25 1.98E-25 ------- $4.31 \mathrm{E}-25 \quad 1.58 \mathrm{E}-25$ ------- 6.46E-25 2.57E-25 ------- 4.31E-25 9.89E-26 ------- 3.59E-25 2.18E-25 ------- 5.75E-25 1.19E-25 ------ 6.46E-25 1.39E-25 -------- -------- ------------- 5.03E-25 ------------- $7.90 \mathrm{E}-25$------------- 3.59E-25 -------------- 7.18E-25 -------------- 3.59E-25 5.94E-26 ------- $1.80 \mathrm{E}-25 \quad 8.91 \mathrm{E}-26$ ------ 3.59E-25 4.95E-26 ------ 1.80E-25 4.95E-26 -------- -------- --------------- -------- $4.62 \mathrm{E}-26$

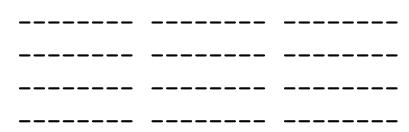


Table 19

The singly differential cross section as a function of the ejected electron energy for double ionization in 5000-25000 $\mathrm{keV} / \mathrm{u} \mathrm{0 \wedge} 3^{+}, 0^{\wedge} 4+$, $0 \wedge 5++$ H_2 collisions.

\begin{tabular}{|c|c|c|c|c|c|c|c|c|c|}
\hline \multirow{2}{*}{$\begin{array}{l}\text { Electron } \\
\text { Energy (eV) }\end{array}$} & \multicolumn{3}{|c|}{ Ion Energy $(\mathrm{keV} / \mathrm{u})$} & \multirow[b]{2}{*}{5000} & \multirow[b]{2}{*}{10000} & \multirow[b]{2}{*}{25000} & \multirow[b]{2}{*}{5000} & \multirow[b]{2}{*}{10000} & \multirow[b]{2}{*}{25000} \\
\hline & 5000 & 10000 & 25000 & & & & & & \\
\hline 0.005 & $\mathrm{E}-19$ & $6.23 \mathrm{E}-20$ & $2.67 \mathrm{E}-20$ & $2.22 \mathrm{E}-19$ & $4 \mathrm{E}-20$ & $2.53 E-20$ & $3.32 \mathrm{E}-19$ & $1.37 \mathrm{E}-19$ & $4.58 \mathrm{E}-20$ \\
\hline 0.030 & $2.53 \mathrm{E}-19$ & $9.31 \mathrm{E}-20$ & $2.97 \mathrm{E}-20$ & $3.50 \mathrm{E}-19$ & $1.29 \mathrm{E}-19$ & $3.79 \mathrm{E}-20$ & $4.92 \mathrm{E}-19$ & $1.79 \mathrm{E}-19$ & $5.12 \mathrm{E}-20$ \\
\hline 0.075 & $3.85 \mathrm{E}-19$ & $1.75 \mathrm{E}-19$ & $4.89 \mathrm{E}-20$ & $5.73 E-19$ & $2.44 \mathrm{E}-19$ & $6.03 \mathrm{E}-20$ & $8.07 \mathrm{E}-19$ & $3.30 \mathrm{E}-19$ & $7.71 \mathrm{E}-20$ \\
\hline 0.150 & $3.73 \mathrm{E}-19$ & $1.72 \mathrm{E}-19$ & $6.14 \mathrm{E}-20$ & $5.30 \mathrm{E}-19$ & $2.47 \mathrm{E}-19$ & $7.65 \mathrm{E}-20$ & $7.97 \mathrm{E}-19$ & 3. $34 \mathrm{E}-19$ & $9.38 \mathrm{E}-20$ \\
\hline 0.350 & $3.61 \mathrm{E}-19$ & $1.70 \mathrm{E}-19$ & $5.63 \mathrm{E}-20$ & $5.21 \mathrm{E}-19$ & $2.29 \mathrm{E}-19$ & $7.03 E-20$ & $7.64 \mathrm{E}-19$ & $3.15 \mathrm{E}-19$ & $8.86 \mathrm{E}-20$ \\
\hline 0.600 & 3.5 & $1.66 \mathrm{E}-19$ & $5.69 \mathrm{E}-20$ & $5.03 \mathrm{E}-19$ & $2.20 \mathrm{E}-19$ & $6.87 \mathrm{E}-20$ & $7.42 \mathrm{E}-19$ & $3.06 \mathrm{E}-19$ & $8.60 \mathrm{E}-20$ \\
\hline 0.850 & $3.40 \mathrm{E}-19$ & $1.58 \mathrm{E}-19$ & $5.62 \mathrm{E}-20$ & $4.92 \mathrm{E}-19$ & $2.14 \mathrm{E}-19$ & $6.58 \mathrm{E}-20$ & $7.17 \mathrm{E}-19$ & $2.91 \mathrm{E}-19$ & $8.34 \mathrm{E}-20$ \\
\hline 1.500 & $3.19 \mathrm{E}-19$ & $1.48 \mathrm{E}-19$ & $4.91 \mathrm{E}-20$ & $4.56 \mathrm{E}-19$ & $1.97 \mathrm{E}-19$ & $6.01 \mathrm{E}-20$ & $6.72 \mathrm{E}-19$ & $2.72 \mathrm{E}-19$ & $7.50 \mathrm{E}-20$ \\
\hline 3.500 & $2.65 \mathrm{E}-19$ & 1. $22 \mathrm{E}-19$ & $4.02 \mathrm{E}-20$ & $3.79 \mathrm{E}-19$ & $1.62 \mathrm{E}-19$ & $4.91 \mathrm{E}-20$ & $5.56 \mathrm{E}-19$ & 2. $23 \mathrm{E}-19$ & $6.14 \mathrm{E}-20$ \\
\hline 6.000 & $2.16 \mathrm{E}-19$ & $9.77 \mathrm{E}-20$ & $3.15 \mathrm{E}-20$ & $3.02 \mathrm{E}-19$ & 1. $28 \mathrm{E}-19$ & $3.86 \mathrm{E}-20$ & $4.45 \mathrm{E}-19$ & $1.74 \mathrm{E}-19$ & $4.68 \mathrm{E}-20$ \\
\hline 8.500 & $1.80 \mathrm{E}-19$ & $8.16 \mathrm{E}-20$ & $2.57 \mathrm{E}-20$ & $.50 \mathrm{E}-19$ & $1.04 \mathrm{E}-19$ & $3.06 \mathrm{E}-20$ & $3.63 \mathrm{E}-19$ & $1.41 \mathrm{E}-19$ & $3.73 \mathrm{E}-20$ \\
\hline 12.500 & 1. $38 \mathrm{E}-19$ & $6.20 \mathrm{E}-20$ & $1.97 \mathrm{E}-20$ & $.91 E-19$ & $7.90 \mathrm{E}-20$ & $2.27 \mathrm{E}-20$ & $2.74 \mathrm{E}$ & $1.05 \mathrm{E}-19$ & $2.73 \mathrm{E}-20$ \\
\hline 17.500 & -19 & $4.59 \mathrm{E}-20$ & $1.46 \mathrm{E}-20$ & $.39 E-19$ & $5.77 \mathrm{E}-20$ & $1.66 \mathrm{E}-20$ & 1.97 & $7.46 \mathrm{E}-20$ & $1.97 \mathrm{E}-20$ \\
\hline 22.500 & 8.1 & $3.55 \mathrm{E}-20$ & $1.09 \mathrm{E}-20$ & $1.09 \mathrm{E}-19$ & $4.37 \mathrm{E}-20$ & $1.27 \mathrm{E}-20$ & $1.48 \mathrm{E}$ & $5.66 \mathrm{E}-20$ & $1.46 \mathrm{E}-20$ \\
\hline 27.500 & 6.5 & $2.86 \mathrm{E}-20$ & $8.88 \mathrm{E}-21$ & $8.57 \mathrm{E}-20$ & $3.49 \mathrm{E}-20$ & $1.00 \mathrm{E}-20$ & $1.16 \mathrm{E}-19$ & $4.34 \mathrm{E}-20$ & $1.15 \mathrm{E}-20$ \\
\hline 32.500 & 5.4 & $2.35 \mathrm{E}-20$ & $6.92 \mathrm{E}-21$ & $6.97 \mathrm{E}-20$ & $2.81 \mathrm{E}-20$ & $8.08 \mathrm{E}-21$ & $9.40 \mathrm{E}-20$ & $3.49 \mathrm{E}-20$ & $9.27 \mathrm{E}-21$ \\
\hline 37.500 & 4.5 & $1.93 \mathrm{E}-20$ & $5.76 \mathrm{E}-21$ & $5.86 \mathrm{E}-20$ & $2.35 \mathrm{E}-20$ & $6.42 \mathrm{E}-21$ & $7.58 \mathrm{E}-20$ & $2.85 \mathrm{E}-20$ & $7.45 \mathrm{E}-21$ \\
\hline 42.500 & $3.85 \mathrm{E}-20$ & $1.63 \mathrm{E}-20$ & $4.93 \mathrm{E}-21$ & $4.94 \mathrm{E}-20$ & $1.97 \mathrm{E}-20$ & $5.55 \mathrm{E}-21$ & $6.45 \mathrm{E}-20$ & $2.41 \mathrm{E}-20$ & $6.21 \mathrm{E}-21$ \\
\hline 47.500 & 3. $35 \mathrm{E}-20$ & $1.43 \mathrm{E}-20$ & $4.16 \mathrm{E}-21$ & $4.27 \mathrm{E}-20$ & $1.69 \mathrm{E}-20$ & $4.65 \mathrm{E}-21$ & $5.52 \mathrm{E}-20$ & $2.05 \mathrm{E}-20$ & $5.37 \mathrm{E}-21$ \\
\hline 52.500 & $2.92 \mathrm{E}-20$ & $1.22 \mathrm{E}-20$ & $3.65 \mathrm{E}-21$ & $3.70 \mathrm{E}-20$ & $1.48 \mathrm{E}-20$ & $4.13 \mathrm{E}-21$ & $4.70 \mathrm{E}-20$ & $1.74 \mathrm{E}-20$ & $4.53 \mathrm{E}-21$ \\
\hline 57.500 & 2.5 & $1.09 \mathrm{E}-20$ & $3.14 \mathrm{E}-21$ & $3.25 \mathrm{E}-20$ & $1.29 \mathrm{E}-20$ & $3.56 \mathrm{E}-21$ & $4.09 \mathrm{E}-20$ & $1.53 \mathrm{E}-20$ & $3.98 \mathrm{E}-21$ \\
\hline 62.500 & 2.2 & $9.50 \mathrm{E}-21$ & $2.79 \mathrm{E}-21$ & $2.90 \mathrm{E}-20$ & $1.13 \mathrm{E}-20$ & $3.13 E-21$ & $3.63 \mathrm{E}-20$ & $1.35 \mathrm{E}-20$ & $3.39 \mathrm{E}-21$ \\
\hline 70.000 & 1.9 & $8.28 \mathrm{E}-21$ & $2.35 \mathrm{E}-21$ & $2.41 \mathrm{E}-20$ & $9.59 \mathrm{E}-21$ & $2.53 \mathrm{E}-21$ & $3.03 \mathrm{E}-20$ & $1.14 \mathrm{E}-20$ & $2.88 \mathrm{E}-21$ \\
\hline 80.000 & -20 & 6.671 & $1.83 \mathrm{E}-21$ & $2.01 \mathrm{E}-20$ & $7.77 \mathrm{E}-21$ & $2.06 \mathrm{E}-21$ & $2.49 \mathrm{E}-20$ & $9.06 \mathrm{E}-21$ & $2.34 \mathrm{E}-21$ \\
\hline 90 & 1.3 & 5.6 & $1.55 \mathrm{E}-21$ & $1.65 \mathrm{E}-20$ & $6.57 \mathrm{E}-21$ & $1.76 \mathrm{E}-21$ & $2.03 \mathrm{E}-20$ & $7.71 \mathrm{E}-21$ & 1. $92 \mathrm{E}-21$ \\
\hline 100 & 1. & 4.7 & $1.36 \mathrm{E}-21$ & $1.40 \mathrm{E}-20$ & $5.58 \mathrm{E}-21$ & $1.44 \mathrm{E}-21$ & $1.70 \mathrm{E}-20$ & $6.35 \mathrm{E}-21$ & $1.56 \mathrm{E}-21$ \\
\hline 11 & 1. & 4.0 & $E-21$ & $1.23 \mathrm{E}-20$ & 4.6 & 1.2 & 1.4 & 5 & 1. $33 \mathrm{E}-21$ \\
\hline 12 & 8.7 & 3.4 & -22 & $1.06 \mathrm{E}-20$ & 4.1 & $1 . C$ & 1.2 & 4. & $1.13 \mathrm{E}-21$ \\
\hline 130 & -21 & 3.1 & -22 & $9.35 \mathrm{E}-21$ & $3.58 \mathrm{E}-21$ & $9.45 \mathrm{E}-22$ & 1.101 & 4.1 & $1.02 \mathrm{E}-21$ \\
\hline 14 & -21 & 2.8 & $E-22$ & $8.37 \mathrm{E}-21$ & $3.20 \mathrm{E}-21$ & $7.90 \mathrm{E}-22$ & $9.73 \mathrm{E}$ & $3.65 \mathrm{E}-21$ & $8.36 \mathrm{E}-22$ \\
\hline 15 & -21 & 2.4 & $E-22$ & $.33 E-21$ & $2.79 \mathrm{E}-21$ & $7.08 \mathrm{E}-22$ & 8.87 & $3.27 \mathrm{E}$ & $7.74 \mathrm{E}-22$ \\
\hline 160.000 & 5.6 & 2.1 & $5.76 \mathrm{E}-22$ & $6.38 \mathrm{E}-21$ & $2.44 \mathrm{E}-21$ & $6.82 \mathrm{E}-22$ & 7.5 & 2.7 & $7.08 \mathrm{E}-22$ \\
\hline 170.000 & 5.0 & 1.95 & $5.68 \mathrm{E}-22$ & $5.93 \mathrm{E}-21$ & $2.20 \mathrm{E}-21$ & $5.72 \mathrm{E}-22$ & 6.96 & $2.50 \mathrm{E}-21$ & $6.43 \mathrm{E}-22$ \\
\hline 187.500 & $4.33 E-21$ & 1.71 & $4.34 \mathrm{E}-22$ & $.11 \mathrm{E}-21$ & $1.88 \mathrm{E}-21$ & $4.85 \mathrm{E}-22$ & 3. $06 \mathrm{E}-21$ & $2.21 \mathrm{E}-21$ & $5.18 \mathrm{E}-22$ \\
\hline 212.500 & 3.47 & 1.36 & $3.59 \mathrm{E}-22$ & $.15 \mathrm{E}-21$ & $1.54 \mathrm{E}-21$ & $3.86 \mathrm{E}-22$ & $.85 \mathrm{E}-21$ & $1.75 \mathrm{E}-21$ & $4.17 \mathrm{E}-22$ \\
\hline 237 & 2.8 & 1.1 & $2.77 \mathrm{E}-22$ & 21 & 1.2 & $E-22$ & 21 & 1.47 & $3.65 \mathrm{E}-22$ \\
\hline 262.500 & $2.48 \mathrm{E}-21$ & $9.59 \mathrm{E}-22$ & $2.46 \mathrm{E}-22$ & $2.86 \mathrm{E}-21$ & $1.04 \mathrm{E}-21$ & $2.56 \mathrm{E}-22$ & $3.36 \mathrm{E}-21$ & 1. $23 \mathrm{E}-21$ & $2.98 \mathrm{E}-22$ \\
\hline 287.500 & $2.12 \mathrm{E}-21$ & $7.94 \mathrm{E}-22$ & $1.96 \mathrm{E}-22$ & $2.49 \mathrm{E}-21$ & $9.14 \mathrm{E}-22$ & $2.29 \mathrm{E}-22$ & $2.86 \mathrm{E}-21$ & $1.02 \mathrm{E}-21$ & $2.46 \mathrm{E}-22$ \\
\hline 312.500 & $1.88 \mathrm{E}-21$ & $6.97 \mathrm{E}-22$ & $1.79 \mathrm{E}-22$ & $2.11 \mathrm{E}-21$ & $7.71 \mathrm{E}-22$ & $1.86 \mathrm{E}-22$ & $2.51 \mathrm{E}-21$ & $8.91 \mathrm{E}-22$ & $1.93 \mathrm{E}-22$ \\
\hline 350.000 & $1.53 \mathrm{E}-21$ & $5.72 \mathrm{E}-22$ & $1.44 \mathrm{E}-22$ & $1.76 \mathrm{E}-21$ & $6.35 \mathrm{E}-22$ & $1.53 \mathrm{E}-22$ & $2.03 \mathrm{E}-21$ & $7.12 \mathrm{E}-22$ & $1.69 \mathrm{E}-22$ \\
\hline 400.000 & 1.2 & $4.44 \mathrm{E}-22$ & $1.08 \mathrm{E}-22$ & 1. $39 \mathrm{E}-21$ & $4.90 \mathrm{E}-22$ & $1.25 \mathrm{E}-22$ & $1.61 \mathrm{E}-21$ & $5.59 \mathrm{E}-22$ & 1. $27 \mathrm{E}-22$ \\
\hline 450.000 & 9.85 & $3.64 \mathrm{E}-22$ & $9.10 \mathrm{E}-23$ & $1.17 \mathrm{E}-21$ & $3.98 \mathrm{E}-22$ & $9.67 \mathrm{E}-23$ & $1.36 \mathrm{E}-21$ & $4.59 \mathrm{E}-22$ & $1.08 \mathrm{E}-22$ \\
\hline 500.000 & 8.0 & 3.02 & $7.76 \mathrm{E}-23$ & $9.25 \mathrm{E}-22$ & $3.16 \mathrm{E}-22$ & $7.66 \mathrm{E}-23$ & $1.10 \mathrm{E}-21$ & $3.76 \mathrm{E}-22$ & $8.59 \mathrm{E}-23$ \\
\hline & -22 & 2.5 & $5.54 \mathrm{E}-23$ & $5-22$ & $2.83 \mathrm{E}-22$ & $6.29 \mathrm{E}-23$ & -22 & $3.12 \mathrm{E}-22$ & $6.93 \mathrm{E}-23$ \\
\hline & 6. & 2.1 & $4.91 \mathrm{E}-23$ & $1 \mathrm{E}-22$ & $2.42 \mathrm{E}-22$ & $5.22 \mathrm{E}-23$ & $86 \mathrm{E}-22$ & $2.71 \mathrm{E}-22$ & $6.01 \mathrm{E}-23$ \\
\hline & & 1.7 & -23 & $E-22$ & $E-22$ & -23 & & & $4.79 \mathrm{E}-23$ \\
\hline & & 1 & -23 & & 1.4 & -23 & & & -23 \\
\hline & & & -23 & -22 & 1.1 & $E-23$ & & -22 & $2.86 \mathrm{E}-23$ \\
\hline & -22 & 8.06 & -23 & $2.87 \mathrm{E}-22$ & $9.90 \mathrm{E}-23$ & $E-23$ & $3.37 \mathrm{E}-22$ & $1 . \mathrm{C}$ & $2.14 \mathrm{E}-23$ \\
\hline 000 & $E-22$ & 7.12 & $1.55 \mathrm{E}-23$ & $2.45 \mathrm{E}-22$ & $7.91 \mathrm{E}-23$ & $1.88 \mathrm{E}-23$ & $2.75 \mathrm{E}-22$ & $9.45 \mathrm{E}-23$ & $1.77 \mathrm{E}-23$ \\
\hline 00 & 1.8 & 5.9 & $1.63 \mathrm{E}-23$ & $2.11 \mathrm{E}-22$ & 6.8 & $E-23$ & $2.32 \mathrm{E}-22$ & 7.4 & $1.73 \mathrm{E}-23$ \\
\hline 1275.000 & $1.51 \mathrm{E}-22$ & $5.43 \mathrm{E}-23$ & $1.08 \mathrm{E}-23$ & $.70 \mathrm{E}-22$ & $5.81 \mathrm{E}-23$ & $1.12 \mathrm{E}-23$ & $1.95 \mathrm{E}-22$ & $6.69 \mathrm{E}-23$ & $1.36 \mathrm{E}-23$ \\
\hline 1375.000 & $1.36 \mathrm{E}-22$ & $4.50 \mathrm{E}-23$ & $1.26 \mathrm{E}-23$ & $1.51 \mathrm{E}-22$ & $4.91 \mathrm{E}-23$ & $1.12 \mathrm{E}-23$ & $1.68 \mathrm{E}-22$ & $5.73 \mathrm{E}-23$ & $1.27 \mathrm{E}-23$ \\
\hline 1475.000 & $1.18 \mathrm{E}-22$ & $4.08 \mathrm{E}-23$ & $9.00 \mathrm{E}-24$ & . $31 \mathrm{E}-22$ & $4.56 \mathrm{E}-23$ & $9.30 \mathrm{E}-24$ & $1.63 \mathrm{E}-22$ & $4.99 \mathrm{E}-23$ & $1.08 \mathrm{E}-23$ \\
\hline 1575.000 & $1.07 \mathrm{E}-22$ & $3.82 \mathrm{E}-23$ & $8.41 \mathrm{E}-24$ & $.23 E-22$ & $4.13 \mathrm{E}-23$ & $8.59 \mathrm{E}-24$ & $1.29 \mathrm{E}-22$ & $4.51 \mathrm{E}-23$ & $9.97 \mathrm{E}-24$ \\
\hline 1675.000 & 9.8 & 3.1 & $5.94 \mathrm{E}-24$ & $.06 \mathrm{E}-22$ & $3.53 \mathrm{E}-23$ & $7.16 \mathrm{E}-24$ & $1.21 \mathrm{E}-22$ & $3.91 \mathrm{E}-23$ & $9.43 \mathrm{E}-24$ \\
\hline 1775.000 & $8.23 E-23$ & 2.7 & $4.85 \mathrm{E}-24$ & $.86 \mathrm{E}-23$ & $2.95 \mathrm{E}-23$ & $8.47 \mathrm{E}-24$ & $.10 \mathrm{E}-22$ & $3.34 \mathrm{H}$ & $8.35 \mathrm{E}-24$ \\
\hline 1875.000 & $7.76 \mathrm{E}-23$ & 2.52 & $6.33 \mathrm{E}-24$ & $8.82 \mathrm{E}-23$ & $2.88 \mathrm{E}-23$ & $6.25 \mathrm{E}-24$ & 9. $91 \mathrm{E}-23$ & $3.11 \mathrm{E}-23$ & $6.84 \mathrm{E}-24$ \\
\hline 1975.000 & $6.35 \mathrm{E}-23$ & $2.20 \mathrm{E}-23$ & $4.95 \mathrm{E}-24$ & $7.70 \mathrm{E}-23$ & $2.89 \mathrm{E}-23$ & $6.37 \mathrm{E}-24$ & $8.86 \mathrm{E}-23$ & $2.83 \mathrm{E}-23$ & $6.20 \mathrm{E}-24$ \\
\hline 2075.000 & $6.63 \mathrm{E}-23$ & $2.17 \mathrm{E}-23$ & $5.84 \mathrm{E}-24$ & $7.36 \mathrm{E}-23$ & $2.32 \mathrm{E}-23$ & $4.79 \mathrm{E}-24$ & $8.02 \mathrm{E}-23$ & $2.44 \mathrm{E}-23$ & $4.53 \mathrm{E}-24$ \\
\hline 2175.000 & $5.52 \mathrm{E}-23$ & $1.80 \mathrm{E}-23$ & $4.45 \mathrm{E}-24$ & $6.37 \mathrm{E}-23$ & $2.07 \mathrm{E}-23$ & $4.67 \mathrm{E}-24$ & $7.37 \mathrm{E}-23$ & $2.50 \mathrm{E}-23$ & $4.63 \mathrm{E}-24$ \\
\hline 2275.000 & $5.19 \mathrm{E}-23$ & $1.63 \mathrm{E}-23$ & $3.36 \mathrm{E}-24$ & $6.21 \mathrm{E}-23$ & $2.08 \mathrm{E}-23$ & $4.59 \mathrm{E}-24$ & $7.38 \mathrm{E}-23$ & $2.28 \mathrm{E}-23$ & $3.93 \mathrm{E}-24$ \\
\hline 2375.000 & $4.67 \mathrm{E}-23$ & $1.56 \mathrm{E}-23$ & $3.86 \mathrm{E}-24$ & $5.28 \mathrm{E}-23$ & $1.75 \mathrm{E}-23$ & $4.63 \mathrm{E}-24$ & $6.13 E-23$ & $1.99 \mathrm{E}-23$ & $3.50 \mathrm{E}-24$ \\
\hline 2475.000 & $4.63 \mathrm{E}-23$ & $1.55 \mathrm{E}-23$ & $2.77 \mathrm{E}-24$ & $4.88 \mathrm{E}-23$ & $1.66 \mathrm{E}-23$ & $4.00 \mathrm{E}-24$ & $5.78 \mathrm{E}-23$ & $1.89 \mathrm{E}-23$ & $3.56 \mathrm{E}-24$ \\
\hline 2575.000 & $4.00 \mathrm{E}-23$ & $1.44 \mathrm{E}-23$ & $2.18 \mathrm{E}-24$ & $5.06 \mathrm{E}-23$ & $1.51 \mathrm{E}-23$ & $3.76 \mathrm{E}-24$ & $5.35 \mathrm{E}-23$ & $1.86 \mathrm{E}-23$ & $3.93 \mathrm{E}-24$ \\
\hline
\end{tabular}


Table 19 (continued)

$2725.000 \quad 3.82 \mathrm{E}-23 \quad 1.22 \mathrm{E}-23 \quad 2.42 \mathrm{E}-24$ $2925.000 \quad 3.14 \mathrm{E}-23 \quad 1.02 \mathrm{E}-23 \quad 2.18 \mathrm{E}-24$ $3125.000 \quad 2.99 \mathrm{E}-23 \quad 8.97 \mathrm{E}-24 \quad 1.83 \mathrm{E}-24$ $\begin{array}{llll}3325.000 & 2.69 \mathrm{E}-23 & 8.18 \mathrm{E}-24 & 1.93 \mathrm{E}-24\end{array}$ $3525.000 \quad 2.38 \mathrm{E}-23 \quad 7.06 \mathrm{E}-24 \quad 1.39 \mathrm{E}-24$ $3725.000 \quad 2.11 \mathrm{E}-23 \quad 6.27 \mathrm{E}-24 \quad 1.43 \mathrm{E}-24$ $3925.000 \quad 1.94 \mathrm{E}-23 \quad 5.78 \mathrm{E}-24 \quad 1.09 \mathrm{E}-24$ $4125.000 \quad 1.72 \mathrm{E}-23 \quad 5.63 \mathrm{E}-24 \quad 1.39 \mathrm{E}-24$ $4325.000 \quad 1.77 \mathrm{E}-23 \quad 4.99 \mathrm{E}-24 \quad 7.42 \mathrm{E}-25$ $4525.000 \quad 1.49 \mathrm{E}-23 \quad 5.18 \mathrm{E}-24 \quad 1.04 \mathrm{E}-24$ $4725.000 \quad 1.25 \mathrm{E}-23 \quad 4.77 \mathrm{E}-24 \quad 7.92 \mathrm{E}-25$ $4925.000 \quad 1.27 \mathrm{E}-23 \quad 4.28 \mathrm{E}-24 \quad 6.93 \mathrm{E}-25$ $5125.000 \quad 1.10 \mathrm{E}-23 \quad 3.53 \mathrm{E}-24 \quad 1.04 \mathrm{E}-24$ $5325.000 \quad 1.13 \mathrm{E}-23 \quad 2.85 \mathrm{E}-24 \quad 7.92 \mathrm{E}-25$ $5525.000 \quad 9.87 \mathrm{E}-24 \quad 3.04 \mathrm{E}-24 \quad 9.40 \mathrm{E}-25$ $5725.000 \quad 9.02 \mathrm{E}-24 \quad 2.89 \mathrm{E}-24 \quad 3.96 \mathrm{E}-25$ $5925.000 \quad 8.22 \mathrm{E}-24 \quad 3.38 \mathrm{E}-24 \quad 3.96 \mathrm{E}-25$ $6125.000 \quad 7.92 \mathrm{E}-24 \quad 2.63 \mathrm{E}-24 \quad 5.44 \mathrm{E}-25$ $6325.000 \quad 7.54 \mathrm{E}-24 \quad 2.59 \mathrm{E}-24 \quad 5.44 \mathrm{E}-25$ $6525.000 \quad 7.54 \mathrm{E}-24 \quad 2.36 \mathrm{E}-24 \quad 4.95 \mathrm{E}-25$ $6875.000 \quad 7.35 \mathrm{E}-24 \quad 1.91 \mathrm{E}-24 \quad 4.55 \mathrm{E}-25$ $\begin{array}{llll}7375.000 & 5.54 \mathrm{E}-24 & 1.59 \mathrm{E}-24 & 2.97 \mathrm{E}-25\end{array}$ $\begin{array}{llll}7875.000 & 4.78 \mathrm{E}-24 & 1.43 \mathrm{E}-24 & 2.97 \mathrm{E}-25\end{array}$ $8375.000 \quad 4.58 \mathrm{E}-24 \quad 1.46 \mathrm{E}-24 \quad 2.57 \mathrm{E}-25$ $8875.000 \quad 4.47 \mathrm{E}-24 \quad 1.14 \mathrm{E}-24 \quad 1.78 \mathrm{E}-25$ $9375.000 \quad 3.54 \mathrm{E}-24 \quad 1.10 \mathrm{E}-24 \quad 1.98 \mathrm{E}-25$ $\begin{array}{llll}9875.000 & 3.46 \mathrm{E}-24 & 9.16 \mathrm{E}-25 & 2.37 \mathrm{E}-25\end{array}$ $\begin{array}{llll}10375.000 & 2.27 \mathrm{E}-24 & 9.46 \mathrm{E}-25 & 2.18 \mathrm{E}-25\end{array}$ $10875.000 \quad 1.36 \mathrm{E}-24 \quad 8.11 \mathrm{E}-25 \quad 1.39 \mathrm{E}-25$ $11375.000 \quad 4.07 \mathrm{E}-25 \quad 7.81 \mathrm{E}-25 \quad 1.78 \mathrm{E}-25$ $11875.000 \quad 1.19 \mathrm{E}-25 \quad 7.96 \mathrm{E}-25 \quad 1.39 \mathrm{E}-25$ 12375.000 ------- $6.75 \mathrm{E}-25$-------12875.000 ------ 6.30E-25 9.89E-26 13375.000 ------ 6.00E-25 $1.58 \mathrm{E}-25$ 13875.000 ------ 6.00E-25 $1.19 \mathrm{E}-25$ 14375.000 ----- $3.75 \mathrm{E}-25 \quad 9.89 \mathrm{E}-26$ 14875.000 15375.000 15875.000 16375.000 17125.000 18125.000 19125.000 20125.000 21125.000 22125.000 23125.000 24125.000 25125.000 26125.000 $\begin{array}{lll}4.41 \mathrm{E}-23 & 1.27 \mathrm{E}-23 & 2.57 \mathrm{E}-24\end{array}$ $\begin{array}{lll}3.47 \mathrm{E}-23 & 1.33 \mathrm{E}-23 & 2.34 \mathrm{E}-24\end{array}$ $\begin{array}{llll}3.49 \mathrm{E}-23 & 9.37 \mathrm{E}-24 & 2.00 \mathrm{E}-24\end{array}$ $2.87 \mathrm{E}-23 \quad 8.76 \mathrm{E}-24 \quad 1.80 \mathrm{E}-24$ $2.68 \mathrm{E}-23 \quad 8.23 \mathrm{E}-24 \quad 1.94 \mathrm{E}-24$ $2.29 \mathrm{E}-23 \quad 6.95 \mathrm{E}-24 \quad 1.54 \mathrm{E}-24$ $2.28 \mathrm{E}-23 \quad 5.95 \mathrm{E}-24 \quad 1.27 \mathrm{E}-24$ $2.01 \mathrm{E}-23 \quad 5.98 \mathrm{E}-24 \quad 1.19 \mathrm{E}-24$ $1.71 \mathrm{E}-23 \quad 4.88 \mathrm{E}-24 \quad 1.37 \mathrm{E}-24$ $1.74 \mathrm{E}-23 \quad 4.77 \mathrm{E}-24 \quad 9.10 \mathrm{E}-25$ $1.55 \mathrm{E}-23 \quad 4.27 \mathrm{E}-24 \quad 7.92 \mathrm{E}-25$ 1.28E-23 $4.31 \mathrm{E}-24 \quad 9.89 \mathrm{E}-25$ 1.30E-23 3.70E-24 8.51E-25 1. 20E-23 3.99E-24 7.52E-25 $1.16 \mathrm{E}-23 \quad 3.35 \mathrm{E}-24 \quad 8.51 \mathrm{E}-25$ 1.16E-23 3.03E-24 6.33E-25 $8.94 \mathrm{E}-24 \quad 2.60 \mathrm{E}-24 \quad 6.93 \mathrm{E}-25$ $9.58 \mathrm{E}-24 \quad 2.71 \mathrm{E}-24 \quad 5.54 \mathrm{E}-25$ $7.51 \mathrm{E}-24 \quad 3.10 \mathrm{E}-24 \quad 4.95 \mathrm{E}-25$ $7.94 \mathrm{E}-24 \quad 2.32 \mathrm{E}-24 \quad 5.74 \mathrm{E}-25$


$6.24 \mathrm{E}-24 \quad 2.22 \mathrm{E}-24 \quad 3.64 \mathrm{E}-25$ 4. 51E-24 1.64E-24 3.48E-25 $5.10 \mathrm{E}-24 \quad 1.70 \mathrm{E}-24 \quad 3.17 \mathrm{E}-25$ 4.51E-24 1.25E-24 2.77E-25 3. $70 \mathrm{E}-24 \quad 1.34 \mathrm{E}-24 \quad 3.25 \mathrm{E}-25$ $3.64 \mathrm{E}-24 \quad 1.08 \mathrm{E}-24 \quad 2.53 \mathrm{E}-25$ $2.58 \mathrm{E}-24 \quad 9.69 \mathrm{E}-25 \quad 1.66 \mathrm{E}-25$

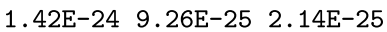
4.02E-25 1.03E-24 $1.98 \mathrm{E}-25$ $1.27 \mathrm{E}-25 \quad 6.98 \mathrm{E}-25 \quad 1.11 \mathrm{E}-25$ ------- 5.84E-25 $1.42 \mathrm{E}-25$ ------ 7.55E-25 7.92E-26 ------ 6.55E-25 $1.19 \mathrm{E}-25$ ------- 7.41E-25 $1.27 \mathrm{E}-25$ ------ 6.41E-25 $1.42 \mathrm{E}-25$ ------ 5.70E-25 1.03E-25

------ 3.13E-25 1.19E-25 ------ $4.84 \mathrm{E}-25 \quad 6.33 \mathrm{E}-26$

------- $4.42 \mathrm{E}-25 \quad 8.71 \mathrm{E}-26$

------- 3.35E-25 9.10E-26

------ 3.13E-25 5.54E-26

------- 2.85E-25 3.96E-26

------- 2.78E-25 4.35E-26

------- $1.64 \mathrm{E}-25 \quad 4.75 \mathrm{E}-26$

------- 7.84E-26 2.77E-26

------- ------- 3.96E-26

------- ------- 5.15E-26

------ ------ $4.75 \mathrm{E}-26$ $\begin{array}{lll}4.85 \mathrm{E}-23 & 1.70 \mathrm{E}-23 & 3.42 \mathrm{E}-24\end{array}$ $\begin{array}{lll}4.13 \mathrm{E}-23 & 1.28 \mathrm{E}-23 & 2.80 \mathrm{E}-24\end{array}$ 3. $52 \mathrm{E}-23 \quad 1.14 \mathrm{E}-23 \quad 2.15 \mathrm{E}-24$ $\begin{array}{lll}3.08 \mathrm{E}-23 & 1.07 \mathrm{E}-23 & 2.37 \mathrm{E}-24\end{array}$ $2.78 \mathrm{E}-23 \quad 7.72 \mathrm{E}-24 \quad 1.97 \mathrm{E}-24$ $2.92 \mathrm{E}-23 \quad 8.32 \mathrm{E}-24 \quad 1.83 \mathrm{E}-24$ $2.51 \mathrm{E}-23 \quad 6.75 \mathrm{E}-24 \quad 1.59 \mathrm{E}-24$ $2.20 \mathrm{E}-23 \quad 6.98 \mathrm{E}-24 \quad 1.08 \mathrm{E}-24$ $2.08 \mathrm{E}-23 \quad 6.51 \mathrm{E}-24 \quad 1.56 \mathrm{E}-24$ $1.96 \mathrm{E}-23 \quad 5.78 \mathrm{E}-24 \quad 9.70 \mathrm{E}-25$ $\begin{array}{ll}1.56 \mathrm{E}-23 & 5.22 \mathrm{E}-24 \\ 1.02 \mathrm{E}-24\end{array}$ 1. $47 \mathrm{E}-23 \quad 5.27 \mathrm{E}-24 \quad 8.08 \mathrm{E}-25$ $\begin{array}{ll}1.47 \mathrm{E}-23 & 5.27 \mathrm{E}-24 \\ 1.29 \mathrm{E}-24\end{array}$ 1.35E-23 3.56E-24 9.16E-25 1. $28 \mathrm{E}-23 \quad 3.70 \mathrm{E}-24 \quad 9.16 \mathrm{E}-25$ 1.12E-23 $3.51 \mathrm{E}-24 \quad 5.12 \mathrm{E}-25$ 1.13E-23 3.05E-24 6.73E-25 9. 38E-24 2.82E-24 4.58E-25 \begin{tabular}{ll}
$8.76 \mathrm{E}-24$ & $2.40 \mathrm{E}-24$ \\
\hline & $8.35 \mathrm{E}-25$
\end{tabular} $1.08 \mathrm{E}-23 \quad 2.73 \mathrm{E}-24 \quad 5.66 \mathrm{E}-25$ $8.87 \mathrm{E}-24 \quad 2.62 \mathrm{E}-24 \quad 4.74 \mathrm{E}-25$ $7.60 \mathrm{E}-24 \quad 2.27 \mathrm{E}-24 \quad 4.96 \mathrm{E}-25$ $\begin{array}{lll}7.54 \mathrm{E}-24 & 2.01 \mathrm{E}-24 & 3.45 \mathrm{E}-25\end{array}$ $5.49 \mathrm{E}-24 \quad 1.52 \mathrm{E}-24 \quad 3.45 \mathrm{E}-25$ $5.21 \mathrm{E}-24 \quad 1.64 \mathrm{E}-24 \quad 3.56 \mathrm{E}-25$ $\begin{array}{lll}4.62 \mathrm{E}-24 & 9.98 \mathrm{E}-25 & 2.37 \mathrm{E}-25\end{array}$ 4. $25 \mathrm{E}-24 \quad 1.29 \mathrm{E}-24 \quad 2.15 \mathrm{E}-25$ $2.95 \mathrm{E}-24 \quad 1.35 \mathrm{E}-24 \quad 2.37 \mathrm{E}-25$ 1. 40E-24 1.09E-24 1.83E-25 $3.41 \mathrm{E}-25 \quad 9.61 \mathrm{E}-25 \quad 1.72 \mathrm{E}-25$ ------- 8.50E-25 1.51E-25 ------- 7.76E-25 1.40E-25 ------- 7.95E-25 1.40E-25 ------- 7.02E-25 2.15E-25 ------- 6.65E-25 1.62E-25 ------ 5.91E-25 $1.08 \mathrm{E}-25$ ------ 6.84E-25 1.19E-25

------ 4.81E-25 9.70E-26 ------- 4.07E-25 7.54E-26 ------ 4.44E-25 6.46E-26 ------- $4.71 \mathrm{E}-25 \quad 9.16 \mathrm{E}-26$ ------ 4.25E-25 7.00E-26 ------ 3.97E-25 5.39E-26 ------ 3.23E-25 2.69E-26 ------ $1.76 \mathrm{E}-25 \quad 4.31 \mathrm{E}-26$ ------- 9.24E-26 3.23E-26 ------- ------- 3.77E-26 ------- ------ 5.39E-26 ------- ------- $5.39 \mathrm{E}-26$ ------ ------ 3.23E-26 
Table 20

The singly differential cross section as a function of the ejected electron energy for double ionization in 5000-25000 keV/u 0^6+, $0^{\wedge} 7+$, $0 \wedge 8++$ H_2 collisions.

\begin{tabular}{|c|c|c|c|c|c|c|c|c|c|}
\hline \multirow{2}{*}{$\begin{array}{l}\text { Electron } \\
\text { Energy (eV) }\end{array}$} & \multicolumn{3}{|c|}{ Ion Energy $(\mathrm{keV} / \mathrm{u})$} & \multirow[b]{2}{*}{5000} & \multirow[b]{2}{*}{10000} & \multirow[b]{2}{*}{25000} & \multirow[b]{2}{*}{5000} & \multirow[b]{2}{*}{10000} & \multirow[b]{2}{*}{25000} \\
\hline & 5000 & 10000 & 25000 & & & & & & \\
\hline 0.005 & $31 \mathrm{E}-19$ & $2.12 \mathrm{E}-19$ & $5.01 \mathrm{E}-20$ & $84 E-19$ & $2.47 \mathrm{E}-19$ & $7.95 \mathrm{E}-20$ & $9.10 \mathrm{E}-19$ & $4.59 \mathrm{E}-19$ & 1. $27 \mathrm{E}-19$ \\
\hline 0.030 & $.99 \mathrm{E}-19$ & $2.71 \mathrm{E}-19$ & $6.52 \mathrm{E}-20$ & $1.08 \mathrm{E}-18$ & 3. $32 \mathrm{E}-19$ & $1.05 \mathrm{E}-19$ & $1.48 \mathrm{E}-18$ & $5.71 \mathrm{E}-19$ & $1.45 \mathrm{E}-19$ \\
\hline 0.075 & 1. $23 \mathrm{E}-18$ & $4.95 \mathrm{E}-19$ & $1.14 \mathrm{E}-19$ & $1.81 \mathrm{E}-18$ & $5.99 \mathrm{E}-19$ & $1.61 \mathrm{E}-19$ & $2.34 \mathrm{E}-18$ & $1.03 \mathrm{E}-18$ & $2.45 \mathrm{E}-19$ \\
\hline 0.150 & $1.21 \mathrm{E}-18$ & $4.92 \mathrm{E}-19$ & 1. $37 \mathrm{E}-19$ & $1.79 \mathrm{E}-18$ & $6.09 \mathrm{E}-19$ & $2.07 \mathrm{E}-19$ & $2.45 \mathrm{E}-18$ & $1.07 \mathrm{E}-18$ & 3. $13 \mathrm{E}-19$ \\
\hline & 1.18 & $4.72 \mathrm{E}-19$ & $1.29 \mathrm{E}-19$ & $1.71 \mathrm{E}-18$ & $5.80 \mathrm{E}-19$ & $1.95 \mathrm{E}-19$ & $2.34 \mathrm{E}-18$ & $1.02 \mathrm{E}-18$ & $2.92 \mathrm{E}-19$ \\
\hline & & $4.51 \mathrm{E}-19$ & 1. $22 \mathrm{E}-19$ & $1.67 \mathrm{E}-18$ & $5.52 \mathrm{E}-19$ & $1.88 \mathrm{E}-19$ & $2.27 \mathrm{E}-18$ & $9.87 \mathrm{E}-19$ & $2.91 \mathrm{E}-19$ \\
\hline & & $4.44 \mathrm{E}-19$ & $1.18 \mathrm{E}-19$ & $1.62 \mathrm{E}-18$ & $5.39 \mathrm{E}-19$ & $1.84 \mathrm{E}-19$ & $2.22 \mathrm{E}-18$ & $9.68 \mathrm{E}-19$ & $2.71 \mathrm{E}-19$ \\
\hline 1.500 & 1.04 & $4.14 \mathrm{E}-19$ & $1.09 \mathrm{E}-19$ & $1.52 \mathrm{E}-18$ & $5.05 \mathrm{E}-19$ & $1.69 \mathrm{E}-19$ & $2.09 \mathrm{E}-18$ & $9.05 \mathrm{E}-19$ & $2.53 E-19$ \\
\hline 3.500 & $8.62 \mathrm{E}-19$ & $3.37 \mathrm{E}-19$ & $8.61 \mathrm{E}-20$ & 1. $29 \mathrm{E}-18$ & $4.18 \mathrm{E}-19$ & 1. $34 \mathrm{E}-19$ & $.79 E-18$ & $7.55 \mathrm{E}-19$ & $2.03 \mathrm{E}-19$ \\
\hline 6.000 & $7.02 \mathrm{E}-19$ & $2.63 \mathrm{E}-19$ & $6.55 \mathrm{E}-20$ & 1. $05 \mathrm{E}-18$ & 3. $33 \mathrm{E}-19$ & $1.02 \mathrm{E}-19$ & $.47 \mathrm{E}-18$ & $6.03 \mathrm{E}-19$ & $1.59 \mathrm{E}-19$ \\
\hline 8.500 & $.75 \mathrm{E}-19$ & $2.11 \mathrm{E}-19$ & $5.16 \mathrm{E}-20$ & $.75 E-19$ & $2.70 \mathrm{E}-19$ & $8.13 E-20$ & $23 \mathrm{E}$ & $4.96 \mathrm{E}-19$ & $1.26 \mathrm{E}-19$ \\
\hline 12.500 & 4.31 & $1.56 \mathrm{E}-19$ & $3.69 \mathrm{E}-20$ & $.65 E-19$ & $1.99 \mathrm{E}-19$ & $5.82 \mathrm{E}-20$ & 19 & $3.70 \mathrm{E}-19$ & \\
\hline 17.500 & 3. $13 \mathrm{E}-19$ & $1.10 \mathrm{E}-19$ & $2.58 \mathrm{E}-20$ & 7. $85 \mathrm{E}-19$ & $1.42 \mathrm{E}-19$ & $4.05 \mathrm{E}-20$ & 19 & 2.6 & \\
\hline 22.500 & 2.3 & $8.10 \mathrm{E}-20$ & $1.86 \mathrm{E}-20$ & $3.68 \mathrm{E}-19$ & $1.05 \mathrm{E}-19$ & $2.93 \mathrm{E}-20$ & & $1.99 \mathrm{E}-19$ & $4.65 \mathrm{E}-20$ \\
\hline 27.500 & 1.82 & $6.24 \mathrm{E}-20$ & $1.44 \mathrm{E}-20$ & $.88 \mathrm{E}-19$ & $8.09 \mathrm{E}-20$ & $2.23 \mathrm{E}-20$ & 19 & $1.53 \mathrm{E}-19$ & $3.50 \mathrm{E}-20$ \\
\hline 32.500 & 1.42 & $4.92 \mathrm{E}-20$ & $1.14 \mathrm{E}-20$ & $2.30 \mathrm{E}-19$ & $6.35 \mathrm{E}-20$ & $1.75 \mathrm{E}-20$ & 3.391 & $1.21 \mathrm{E}$ & $2.74 \mathrm{E}-20$ \\
\hline 37. & 1.1 & $3.99 \mathrm{E}-20$ & $9.15 \mathrm{E}-21$ & $1.88 \mathrm{E}-19$ & $5.04 \mathrm{E}-20$ & $1.40 \mathrm{E}-20$ & $2.75 \mathrm{E}$ & $9.81 \mathrm{E}-20$ & $2.24 \mathrm{E}-20$ \\
\hline 42.500 & 9.57 & $3.29 \mathrm{E}-20$ & $7.56 \mathrm{E}-21$ & $1.54 \mathrm{E}-19$ & $4.20 \mathrm{E}-20$ & $1.15 \mathrm{E}-20$ & $2.30 \mathrm{E}$ & $8.04 \mathrm{E}-20$ & $1.76 \mathrm{E}-20$ \\
\hline 47.500 & $8.09 \mathrm{E}-20$ & $2.70 \mathrm{E}-20$ & $6.27 \mathrm{E}-21$ & 1. $30 \mathrm{E}-19$ & $3.48 \mathrm{E}-20$ & $9.50 \mathrm{E}-21$ & $1.93 \mathrm{E}$ & $6.76 \mathrm{E}-20$ & $1.49 \mathrm{E}-20$ \\
\hline 52.500 & $6.85 \mathrm{E}-20$ & $2.33 \mathrm{E}-20$ & $5.38 \mathrm{E}-21$ & $1.10 \mathrm{E}-19$ & $2.98 \mathrm{E}-20$ & $8.18 \mathrm{E}-21$ & $1.66 \mathrm{I}$ & $5.70 \mathrm{E}-20$ & $1.25 \mathrm{E}-20$ \\
\hline 57.500 & 5.83 & $2.00 \mathrm{E}-20$ & $4.74 \mathrm{E}-21$ & $9.54 \mathrm{E}-20$ & $2.49 \mathrm{E}-20$ & $6.76 \mathrm{E}-21$ & 1.43 & $4.97 \mathrm{E}-20$ & $1.06 \mathrm{E}-20$ \\
\hline 62.500 & 5.12 & $1.76 \mathrm{E}-20$ & $4.11 \mathrm{E}-21$ & $8.21 \mathrm{E}-20$ & $2.17 \mathrm{E}-20$ & $5.78 \mathrm{E}-21$ & 1.23 & $4.27 \mathrm{E}-20$ & $9.11 \mathrm{E}-21$ \\
\hline 70. & 4.22 & $1.43 \mathrm{E}-20$ & $3.37 \mathrm{E}-21$ & $6.76 \mathrm{E}-20$ & $1.79 \mathrm{E}-20$ & $4.79 \mathrm{E}-21$ & 1.031 & $3.46 \mathrm{E}-20$ & $7.49 \mathrm{E}-21$ \\
\hline 80. & 3.35 & $1.16 \mathrm{E}-20$ & $2.72 \mathrm{E}-21$ & $5.34 \mathrm{E}-20$ & $1.41 \mathrm{E}-20$ & $3.73 \mathrm{E}-21$ & 8.131 & $2.76 \mathrm{E}-20$ & $5.74 \mathrm{E}-21$ \\
\hline 90 & 2.7 & 9.51 & $2.17 \mathrm{E}-21$ & 4.28 & $1.15 \mathrm{E}-20$ & $3.03 E-21$ & 6.58 & $2.21 \mathrm{E}-20$ & $4.75 \mathrm{E}-21$ \\
\hline 100 & 2.2 & 8.04 & $1.87 \mathrm{E}-21$ & $3.57 \mathrm{E}-20$ & $9.47 \mathrm{E}-21$ & $2.49 \mathrm{E}-21$ & 5.41 & $1.83 \mathrm{E}$ & $3.94 \mathrm{E}-21$ \\
\hline & 1.9 & 6.6 & $1.51 \mathrm{E}-21$ & 2.9 & $7.77 \mathrm{E}-21$ & 2.1 & 20 & 1.5 & $E-21$ \\
\hline 12 & 1.6 & 5.8 & $E-21$ & 2.53 & $6.57 \mathrm{E}-21$ & -21 & 20 & 1.2 & $2.73 E-21$ \\
\hline 13 & 1.43 & 4.99 & $1.14 \mathrm{E}-21$ & 2.18 & $5.69 \mathrm{E}-21$ & 1.54 & 20 & $1.10 \mathrm{~F}$ & $2.32 \mathrm{E}-21$ \\
\hline 14 & 1.25 & 4. $33 \mathrm{E}-21$ & $1.02 \mathrm{E}-21$ & -20 & $4.98 \mathrm{E}-21$ & $1.36 \mathrm{E}-21$ & 87 & 9.71 & $2.01 \mathrm{E}-21$ \\
\hline 15 & $1.1 \mathrm{C}$ & $3.82 \mathrm{E}-21$ & $8.75 E-22$ & -20 & $4.41 \mathrm{E}-21$ & 1. $21 \mathrm{E}-21$ & 20 & 8.5 & $1.76 \mathrm{E}-21$ \\
\hline 160.000 & -21 & $3.41 \mathrm{E}-21$ & $7.88 \mathrm{E}-22$ & $42 \mathrm{E}-20$ & $3.83 \mathrm{E}-21$ & $1.02 \mathrm{E}-21$ & 20 & 7.28 & $1.64 \mathrm{E}-21$ \\
\hline 170.000 & $8.81 \mathrm{E}-21$ & $2.98 \mathrm{E}-21$ & $7.14 \mathrm{E}-22$ & $30 \mathrm{E}-20$ & $3.40 \mathrm{E}-21$ & $9.97 \mathrm{E}-22$ & 20 & 6.46 & $1.36 \mathrm{E}-21$ \\
\hline 187.500 & $7.62 \mathrm{E}-21$ & $2.64 \mathrm{E}-21$ & $5.97 \mathrm{E}-22$ & $.08 E-20$ & $2.89 \mathrm{E}-21$ & $7.90 \mathrm{E}-22$ & 65 & $5.50 \mathrm{E}-21$ & $1.13 \mathrm{E}-21$ \\
\hline 212.500 & $5.94 \mathrm{E}-21$ & $2.09 \mathrm{E}-21$ & $5.04 \mathrm{E}-22$ & $55 \mathrm{E}-21$ & $2.27 \mathrm{E}-21$ & $5.95 \mathrm{E}-22$ & 301 & $4.29 \mathrm{E}-21$ & $8.85 \mathrm{E}-22$ \\
\hline 237 & 4.9 & 1.71 & $3.97 \mathrm{E}-22$ & 21 & $1.80 \mathrm{E}-21$ & 5.06 & 20 & 3. & 7. $32 \mathrm{E}-22$ \\
\hline 262.500 & 4.16 & $1.45 \mathrm{E}-21$ & $3.27 \mathrm{E}-22$ & $5.66 \mathrm{E}-21$ & $1.54 \mathrm{E}-21$ & $4.16 \mathrm{E}-22$ & $8.60 \mathrm{E}$ & $2.82 \mathrm{E}-21$ & $5.70 \mathrm{E}-22$ \\
\hline 287.500 & $3.48 \mathrm{E}-21$ & 1. $23 \mathrm{E}-21$ & $2.72 \mathrm{E}-22$ & $4.80 \mathrm{E}-21$ & $1.28 \mathrm{E}-21$ & $3.56 \mathrm{E}-22$ & $7.20 \mathrm{E}$ & $2.37 \mathrm{E}-21$ & $5.23 \mathrm{E}-22$ \\
\hline 312.500 & $3.05 \mathrm{E}-21$ & $1.09 \mathrm{E}-21$ & $2.60 \mathrm{E}-22$ & $4.06 \mathrm{E}-21$ & $1.10 \mathrm{E}-21$ & $2.92 \mathrm{E}-22$ & 6.16 & $1.96 \mathrm{E}-21$ & $4.01 \mathrm{E}-22$ \\
\hline 350.000 & $2.48 \mathrm{E}-21$ & $8.53 \mathrm{E}-22$ & $1.91 \mathrm{E}-22$ & 3. $34 \mathrm{E}-21$ & $8.89 \mathrm{E}-22$ & $2.34 \mathrm{E}-22$ & 4.80 & $1.61 \mathrm{E}-21$ & $3.29 \mathrm{E}-22$ \\
\hline 400.000 & 1.94 & $6.86 \mathrm{E}-22$ & $1.54 \mathrm{E}-22$ & $2.52 \mathrm{E}-21$ & $6.83 \mathrm{E}-22$ & $1.79 \mathrm{E}-22$ & 3.74 & 1. $24 \mathrm{E}-21$ & $2.65 \mathrm{E}-22$ \\
\hline 450.000 & 1.57 & 5.50 & $1.19 \mathrm{E}-22$ & $2.01 \mathrm{E}-21$ & $5.49 \mathrm{E}-22$ & $1.45 \mathrm{E}-22$ & 3.01 & $1.00 \mathrm{E}-21$ & $2.07 \mathrm{E}-22$ \\
\hline 500.000 & 1.3 & 4.75 & $9.19 \mathrm{E}-23$ & $1.71 \mathrm{E}-21$ & $4.60 \mathrm{E}-22$ & 1. $19 \mathrm{E}-22$ & 21 & $7.76 \mathrm{E}-22$ & $1.63 \mathrm{E}-22$ \\
\hline & 1.1 & 3.7 & $8.25 E-23$ & 1.4 & $3.82 \mathrm{E}-22$ & 9.48 & & $6.59 \mathrm{E}-22$ & $1.31 \mathrm{E}-22$ \\
\hline & & 3.1 & $E-23$ & $E-21$ & $3.23 \mathrm{E}-22$ & 8.42 & & -22 & $1.13 \mathrm{E}-22$ \\
\hline & & 2.6 & -23 & & 2.51 & 6.66 & & & E-23 \\
\hline & & $1 . s$ & -23 & & 2.0 & 5.2 & & & -23 \\
\hline & & & -23 & & 1.58 & 4.2 & & 2.4 & -23 \\
\hline 0 & 3.8 & 1.2 & -23 & 22 & 1.23E-22 & $3.43^{3}$ & & 2.1 & $4.42 \mathrm{E}-23$ \\
\hline 1075.000 & 3.35 & $1.10 \mathrm{E}-22$ & -23 & $3.91 \mathrm{E}-22$ & $1.01 \mathrm{E}-22$ & $2.65 \mathrm{E}-23$ & 22 & 1.6 & $3.67 \mathrm{E}-23$ \\
\hline 117 & 2.68 & $8.93 \mathrm{E}-23$ & $E-23$ & -22 & $8.73 E-23$ & $2.20 \mathrm{E}-23$ & & 1.4 & $2.86 \mathrm{E}-23$ \\
\hline 1275.000 & $2.41 \mathrm{E}$ & $8.22 \mathrm{E}-23$ & $1.65 \mathrm{E}-23$ & $.81 E-22$ & $7.57 \mathrm{E}-23$ & $1.89 \mathrm{E}-23$ & 22 & 1.1 & $2.75 \mathrm{E}-23$ \\
\hline 1375.000 & $2.14 \mathrm{E}-22$ & $6.59 \mathrm{E}-23$ & $1.34 \mathrm{E}-23$ & $45 \mathrm{E}-22$ & $6.84 \mathrm{E}-23$ & $1.60 \mathrm{E}-23$ & . $31 E-22$ & $1.11 \mathrm{E}-22$ & $1.93 \mathrm{E}-23$ \\
\hline 1475.000 & 1. $87 \mathrm{E}-22$ & $5.72 \mathrm{E}-23$ & $1.20 \mathrm{E}-23$ & $17 E-22$ & $5.70 \mathrm{E}-23$ & 1. $39 \mathrm{E}-23$ & $84 E-22$ & $8.70 \mathrm{E}-23$ & $1.68 \mathrm{E}-23$ \\
\hline 1575.000 & $1.71 \mathrm{E}-22$ & $5.09 \mathrm{E}-23$ & $1.03 \mathrm{E}-23$ & $91 \mathrm{E}-22$ & $4.91 \mathrm{E}-23$ & 1. $33 \mathrm{E}-23$ & $.46 \mathrm{E}$ & $8.29 \mathrm{E}-23$ & $1.62 \mathrm{E}-23$ \\
\hline 1675 & 1.43 & 4.71 & -24 & 62 & $4.26 \mathrm{E}-23$ & $9.92 \mathrm{E}-24$ & 08 & $7.15 \mathrm{E}$ & $1.68 \mathrm{E}-23$ \\
\hline 1775.000 & 1.34 & 3.83 & -24 & 58 & $3.62 \mathrm{E}-23$ & $1.01 \mathrm{I}$ & .95 & 6.26 & $1.27 \mathrm{E}-23$ \\
\hline 1875.000 & $1.19 \mathrm{E}-22$ & $3.83 \mathrm{E}-23$ & $7.54 \mathrm{E}-24$ & $1.31 \mathrm{E}-22$ & $3.47 \mathrm{E}-23$ & $8.02 \mathrm{E}-24$ & $1.68 \mathrm{E}$ & $5.56 \mathrm{E}-23$ & $1.22 \mathrm{E}-23$ \\
\hline 1975.000 & $1.05 \mathrm{E}-22$ & $3.50 \mathrm{E}-23$ & $8.46 \mathrm{E}-24$ & $1.16 \mathrm{E}-22$ & $3.30 \mathrm{E}-23$ & $8.23 E-24$ & $1.61 \mathrm{E}-22$ & $5.37 \mathrm{E}-23$ & $1.01 \mathrm{E}-23$ \\
\hline 2075.000 & $9.96 \mathrm{E}-23$ & $2.85 \mathrm{E}-23$ & $5.82 \mathrm{E}-24$ & $1.12 \mathrm{E}-22$ & $2.92 \mathrm{E}-23$ & $7.88 \mathrm{E}-24$ & $1.41 \mathrm{E}-22$ & $4.73 \mathrm{E}-23$ & $9.29 \mathrm{E}-24$ \\
\hline 2175.000 & $9.15 \mathrm{E}-23$ & $2.73 \mathrm{E}-23$ & $5.93 \mathrm{E}-24$ & $1.02 \mathrm{E}-22$ & $2.57 \mathrm{E}-23$ & $6.83 \mathrm{E}-24$ & 1. $37 \mathrm{E}-22$ & $4.71 \mathrm{E}-23$ & $8.47 \mathrm{E}-24$ \\
\hline 2275.000 & $7.53 \mathrm{E}-23$ & $2.72 \mathrm{E}-23$ & $4.63 \mathrm{E}-24$ & $9.10 \mathrm{E}-23$ & $2.27 \mathrm{E}-23$ & $5.42 \mathrm{E}-24$ & $1.09 \mathrm{E}-22$ & $3.97 \mathrm{E}-23$ & $9.56 \mathrm{E}-24$ \\
\hline 2375.000 & $7.95 \mathrm{E}-23$ & $2.24 \mathrm{E}-23$ & $5.17 \mathrm{E}-24$ & $8.87 E-23$ & $2.48 \mathrm{E}-23$ & $4.71 \mathrm{E}-24$ & $1.07 \mathrm{E}-22$ & $3.37 \mathrm{E}-23$ & $8.01 \mathrm{E}-24$ \\
\hline 2475.000 & $6.05 \mathrm{E}-23$ & $2.31 \mathrm{E}-23$ & $4.58 \mathrm{E}-24$ & $7.62 \mathrm{E}-23$ & $1.79 \mathrm{E}-23$ & $4.15 \mathrm{E}-24$ & $1.01 \mathrm{E}-22$ & $3.81 \mathrm{E}-23$ & $6.28 \mathrm{E}-24$ \\
\hline 2575.000 & $6.81 \mathrm{E}-23$ & $2.04 \mathrm{E}-23$ & $4.09 \mathrm{E}-24$ & $7.23 \mathrm{E}-23$ & $1.86 \mathrm{E}-23$ & $4.64 \mathrm{E}-24$ & $9.52 \mathrm{E}-23$ & 3. $33 \mathrm{E}-23$ & $7.56 \mathrm{E}-24$ \\
\hline
\end{tabular}


Table 20 (continued)

$2725.000 \quad 5.83 \mathrm{E}-23 \quad 1.65 \mathrm{E}-23 \quad 3.66 \mathrm{E}-24$ $2925.000 \quad 4.87 \mathrm{E}-23 \quad 1.64 \mathrm{E}-23 \quad 3.12 \mathrm{E}-24$ $3125.000 \quad 4.57 \mathrm{E}-23 \quad 1.34 \mathrm{E}-23 \quad 2.75 \mathrm{E}-24$ $\begin{array}{llll}3325.000 & 4.00 \mathrm{E}-23 & 1.25 \mathrm{E}-23 & 2.18 \mathrm{E}-24\end{array}$


$3725.000 \quad 3.25 \mathrm{E}-23 \quad 1.09 \mathrm{E}-23 \quad 2.07 \mathrm{E}-24$ $3925.000 \quad 2.95 \mathrm{E}-23 \quad 8.18 \mathrm{E}-24 \quad 1.75 \mathrm{E}-24$ $4125.000 \quad 2.67 \mathrm{E}-23 \quad 7.47 \mathrm{E}-24 \quad 1.29 \mathrm{E}-24$ $4325.000 \quad 2.36 \mathrm{E}-23 \quad 7.42 \mathrm{E}-24 \quad 1.51 \mathrm{E}-24$ $4525.000 \quad 2.14 \mathrm{E}-23 \quad 6.71 \mathrm{E}-24 \quad 1.32 \mathrm{E}-24$

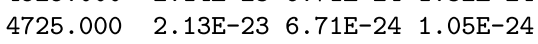
$4925.000 \quad 1.91 \mathrm{E}-23 \quad 5.18 \mathrm{E}-24 \quad 1.21 \mathrm{E}-24$ $5125.000 \quad 1.66 \mathrm{E}-23 \quad 4.83 \mathrm{E}-24 \quad 1.13 \mathrm{E}-24$ $5325.000 \quad 1.50 \mathrm{E}-23 \quad 4.78 \mathrm{E}-24 \quad 1.10 \mathrm{E}-24$ $5525.000 \quad 1.55 \mathrm{E}-23 \quad 4.98 \mathrm{E}-24 \quad 6.20 \mathrm{E}-25$ $5725.000 \quad 1.53 \mathrm{E}-23 \quad 4.62 \mathrm{E}-24 \quad 7.27 \mathrm{E}-25$ $5925.000 \quad 1.29 \mathrm{E}-23 \quad 3.10 \mathrm{E}-24 \quad 9.16 \mathrm{E}-25$ $6125.000 \quad 1.37 \mathrm{E}-23 \quad 3.66 \mathrm{E}-24 \quad 8.08 \mathrm{E}-25$ $6325.000 \quad 1.17 \mathrm{E}-23 \quad 3.76 \mathrm{E}-24 \quad 5.66 \mathrm{E}-25$ $6525.000 \quad 1.14 \mathrm{E}-23 \quad 3.46 \mathrm{E}-24 \quad 6.46 \mathrm{E}-25$ $6875.000 \quad 9.08 \mathrm{E}-24 \quad 3.29 \mathrm{E}-24 \quad 6.25 \mathrm{E}-25$ $\begin{array}{llll}7375.000 & 8.02 \mathrm{E}-24 & 2.05 \mathrm{E}-24 & 5.49 \mathrm{E}-25\end{array}$ $\begin{array}{llll}7875.000 & 7.57 \mathrm{E}-24 & 2.44 \mathrm{E}-24 & 4.85 \mathrm{E}-25\end{array}$ $8375.000 \quad 6.26 \mathrm{E}-24 \quad 1.69 \mathrm{E}-24 \quad 3.66 \mathrm{E}-25$ $8875.000 \quad 5.77 \mathrm{E}-24 \quad 1.61 \mathrm{E}-24 \quad 3.56 \mathrm{E}-25$ $9375.000 \quad 5.63 \mathrm{E}-24 \quad 1.48 \mathrm{E}-24 \quad 3.34 \mathrm{E}-25$ $9875.000 \quad 3.98 \mathrm{E}-24 \quad 1.57 \mathrm{E}-24 \quad 3.02 \mathrm{E}-25$ $10375.000 \quad 2.67 \mathrm{E}-24 \quad 1.44 \mathrm{E}-24 \quad 3.56 \mathrm{E}-25$ $10875.000 \quad 1.76 \mathrm{E}-24 \quad 1.30 \mathrm{E}-24 \quad 2.15 \mathrm{E}-25$ $11375.000 \quad 4.93 \mathrm{E}-25 \quad 9.76 \mathrm{E}-25 \quad 1.51 \mathrm{E}-25$ 11875.000 ------- 9.15E-25 $1.62 \mathrm{E}-25$ 12375.000 ------ 9.96E-25 $1.72 \mathrm{E}-25$ 12875.000 ------ 9.15E-25 2.15E-25 13375.000 ------ 9.15E-25 $1.29 \mathrm{E}-25$ 13875.000 ------- 7.11E-25 $1.40 \mathrm{E}-25$ 14375.000 ------ 6.30E-25 $1.62 \mathrm{E}-25$ 14875.000 ------- 8.74E-25 1.62E-25 15375.000 ------- 5.29E-25 $1.40 \mathrm{E}-25$ 15875.000 ----- 6.50E-25 $1.08 \mathrm{E}-25$ 16375.000 ------ $4.88 \mathrm{E}-25 \quad 6.46 \mathrm{E}-26$ 17125.000 ------- $6.00 \mathrm{E}-25 \quad 1.19 \mathrm{E}-25$ 18125.000 ------ 2.74E-25 8.08E-26 19125.000 ------ $4.68 \mathrm{E}-25 \quad 7.54 \mathrm{E}-26$ 20125.000 ------ 4.98E-25 8.08E-26 21125.000 ------ 2.74E-25 $6.46 \mathrm{E}-26$ 22125.000 ------- $1.22 \mathrm{E}-25 \quad 6.46 \mathrm{E}-26$ 23125.000 -------- ------- 4.85E-26 24125.000 -------- -------- 7.54E-26 25125.000 -------- ------- $4.85 \mathrm{E}-26$ 26125.000
$6.88 \mathrm{E}-23 \quad 1.70 \mathrm{E}-23 \quad 4.40 \mathrm{E}-24$ $5.77 \mathrm{E}-23 \quad 1.51 \mathrm{E}-23 \quad 3.41 \mathrm{E}-24$ $\begin{array}{llll}5.51 \mathrm{E}-23 & 1.32 \mathrm{E}-23 & 3.24 \mathrm{E}-24\end{array}$ 4. 32E-23 1.19E-23 3.13E-24 $\begin{array}{lll}4.20 \mathrm{E}-23 & 1.01 \mathrm{E}-23 & 2.74 \mathrm{E}-24\end{array}$ 3. $62 \mathrm{E}-23 \quad 7.57 \mathrm{E}-24 \quad 2.36 \mathrm{E}-24$ $\begin{array}{ll}3.80 \mathrm{E}-23 & 8.84 \mathrm{E}-24 \\ 2.39 \mathrm{E}-24\end{array}$ 3. $10 \mathrm{E}-23 \quad 8.08 \mathrm{E}-24 \quad 2.01 \mathrm{E}-24$ $2.70 \mathrm{E}-23 \quad 6.35 \mathrm{E}-24 \quad 1.69 \mathrm{E}-24$ $2.70 \mathrm{E}-23 \quad 6.81 \mathrm{E}-24 \quad 1.79 \mathrm{E}-24$ $2.41 \mathrm{E}-23 \quad 5.79 \mathrm{E}-24 \quad 1.30 \mathrm{E}-24$ $2.16 \mathrm{E}-23 \quad 5.08 \mathrm{E}-24 \quad 1.16 \mathrm{E}-24$ $2.01 \mathrm{E}-23 \quad 5.13 \mathrm{E}-24 \quad 1.09 \mathrm{E}-24$ $1.89 \mathrm{E}-23 \quad 4.42 \mathrm{E}-24 \quad 8.09 \mathrm{E}-25$ $\begin{array}{lll}1.72 \mathrm{E}-23 & 4.47 \mathrm{E}-24 & 1.20 \mathrm{E}-24\end{array}$ $1.60 \mathrm{E}-23 \quad 4.17 \mathrm{E}-24 \quad 9.15 \mathrm{E}-25$ $1.73 \mathrm{E}-23 \quad 3.51 \mathrm{E}-24 \quad 1.16 \mathrm{E}-24$ $1.43 \mathrm{E}-23 \quad 3.91 \mathrm{E}-24 \quad 6.68 \mathrm{E}-25$ $1.33 \mathrm{E}-23 \quad 3.46 \mathrm{E}-24 \quad 4.57 \mathrm{E}-25$ $1.33 \mathrm{E}-23 \quad 3.20 \mathrm{E}-24 \quad 6.68 \mathrm{E}-25$ $1.08 \mathrm{E}-23 \quad 2.48 \mathrm{E}-24 \quad 6.19 \mathrm{E}-25$ $\begin{array}{lll}8.98 \mathrm{E}-24 & 2.40 \mathrm{E}-24 & 4.22 \mathrm{E}-25\end{array}$ 7.54E-24 1.93E-24 6.47E-25 8. 24E-24 1.63E-24 6.05E-25 $6.71 \mathrm{E}-24 \quad 1.77 \mathrm{E}-24 \quad 5.35 \mathrm{E}-25$ $6.51 \mathrm{E}-24 \quad 1.42 \mathrm{E}-24 \quad 3.66 \mathrm{E}-25$ 4. 36E-24 1.48E-24 3.38E-25 $3.58 \mathrm{E}-24 \quad 1.38 \mathrm{E}-24 \quad 3.10 \mathrm{E}-25$ $2.64 \mathrm{E}-24 \quad 1.34 \mathrm{E}-24 \quad 2.67 \mathrm{E}-25$ $4.53 \mathrm{E}-25 \quad 8.33 \mathrm{E}-25 \quad 1.27 \mathrm{E}-25$ ------- 8.74E-25 $1.83 \mathrm{E}-25$ ------- 7.52E-25 2.11E-25 ------- 9.55E-25 1.55E-25 ------- 7.52E-25 2.25E-25 ------- 7.11E-25 1.69E-25 ------ 5.69E-25 1.83E-25 ------ 6.50E-25 9.85E-26 ------ 7.11E-25 9.85E-26 ------- 6.10E-25 1.41E-25 ------- 6.10E-25 9.85E-26 ------- $3.76 \mathrm{E}-25 \quad 1.55 \mathrm{E}-25$ ------ 4.57E-25 5.63E-26 ------ 3.56E-25 9.15E-26 ------- 2.64E-25 9.15E-26 ------- 2.13E-25 5.63E-26 ------- $7.11 \mathrm{E}-26 \quad 4.22 \mathrm{E}-26$ -------- ------ $4.22 \mathrm{E}-26$ ------- ------- 6.33E-26 ------- ------ $4.22 \mathrm{E}-26$ -------- ------- $4.93 \mathrm{E}-26$ $\begin{array}{lll}7.67 \mathrm{E}-23 & 2.88 \mathrm{E}-23 & 6.24 \mathrm{E}-24\end{array}$ $\begin{array}{lll}7.15 \mathrm{E}-23 & 2.32 \mathrm{E}-23 & 4.69 \mathrm{E}-24\end{array}$ $\begin{array}{llll}6.13 \mathrm{E}-23 & 2.00 \mathrm{E}-23 & 3.87 \mathrm{E}-24\end{array}$ $5.77 \mathrm{E}-23 \quad 1.68 \mathrm{E}-23 \quad 3.91 \mathrm{E}-24$ $5.17 \mathrm{E}-23 \quad 1.50 \mathrm{E}-23 \quad 2.96 \mathrm{E}-24$ $\begin{array}{ll}4.60 \mathrm{E}-23 & 1.45 \mathrm{E}-23 \\ 2.32 \mathrm{E}-24\end{array}$ $\begin{array}{lll}4.15 \mathrm{E}-23 & 1.23 \mathrm{E}-23 & 2.41 \mathrm{E}-24\end{array}$ 3. $82 \mathrm{E}-23 \quad 1.14 \mathrm{E}-23 \quad 1.96 \mathrm{E}-24$ 3. $12 \mathrm{E}-23 \quad 1.09 \mathrm{E}-23 \quad 1.96 \mathrm{E}-24$ 3. $41 \mathrm{E}-23 \quad 1.14 \mathrm{E}-23 \quad 2.50 \mathrm{E}-24$ $\begin{array}{lll}2.74 \mathrm{E}-23 & 9.70 \mathrm{E}-24 & 1.41 \mathrm{E}-24\end{array}$ $2.65 \mathrm{E}-23 \quad 9.63 \mathrm{E}-24 \quad 2.28 \mathrm{E}-24$ $2.69 \mathrm{E}-23 \quad 7.39 \mathrm{E}-24 \quad 1.73 \mathrm{E}-24$ 2. $31 \mathrm{E}-23 \quad 6.27 \mathrm{E}-24 \quad 1.14 \mathrm{E}-24$ $\begin{array}{lll}1.95 \mathrm{E}-23 & 7.46 \mathrm{E}-24 & 1.32 \mathrm{E}-24\end{array}$ $1.68 \mathrm{E}-23 \quad 5.15 \mathrm{E}-24 \quad 8.65 \mathrm{E}-25$ $1.70 \mathrm{E}-23 \quad 5.94 \mathrm{E}-24 \quad 1.18 \mathrm{E}-24$ $1.54 \mathrm{E}-23 \quad 5.08 \mathrm{E}-24 \quad 8.65 \mathrm{E}-25$ $1.89 \mathrm{E}-23 \quad 6.14 \mathrm{E}-24 \quad 1.14 \mathrm{E}-24$ $1.43 \mathrm{E}-23 \quad 4.22 \mathrm{E}-24 \quad 1.14 \mathrm{E}-24$ 1. $26 \mathrm{E}-23 \quad 4.51 \mathrm{E}-24 \quad 1.04 \mathrm{E}-24$ $1.04 \mathrm{E}-23 \quad 4.17 \mathrm{E}-24 \quad 7.65 \mathrm{E}-25$ 9.52E-24 2.93E-24 5.46E-25 8.68E-24 2.85E-24 5.46E-25 $8.68 \mathrm{E}-24 \quad 2.64 \mathrm{E}-24 \quad 3.82 \mathrm{E}-25$ $6.90 \mathrm{E}-24 \quad 2.11 \mathrm{E}-24 \quad 5.83 \mathrm{E}-25$ $6.66 \mathrm{E}-24 \quad 1.93 \mathrm{E}-24 \quad 6.01 \mathrm{E}-25$ $5.28 \mathrm{E}-24 \quad 1.90 \mathrm{E}-24 \quad 4.19 \mathrm{E}-25$ $2.17 \mathrm{E}-24 \quad 2.35 \mathrm{E}-24 \quad 4.19 \mathrm{E}-25$ $1.04 \mathrm{E}-24 \quad 1.43 \mathrm{E}-24 \quad 2.37 \mathrm{E}-25$ ------- 1.37E-24 2.55E-25

------- $1.21 \mathrm{E}-24 \quad 2.91 \mathrm{E}-25$ ------- 1.40E-24 2.00E-25 ------- 1.32E-24 2.00E-25 ------ 1.03E-24 2.18E-25 ------ 9.77E-25 2.00E-25 ------- 7.13E-25 1.46E-25 ------ 8.45E-25 2.91E-25 ------- 6.86E-25 1.82E-25 ------- $7.66 \mathrm{E}-25 \quad 1.27 \mathrm{E}-25$ ------- 6.07E-25 1.82E-25 ------- 7.52E-25 1.37E-25 ------ 5.54E-25 1.00E-25 ----- 6.60E-25 1.00E-25 ------ 3.43E-25 6.37E-26 ------- 1.32E-25 1.37E-25 ------- ------- 5.46E-26 ------- ------- -------

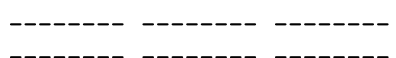


Table 21

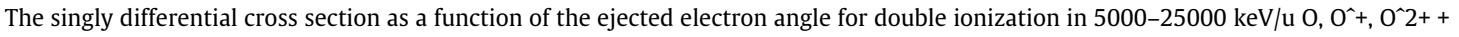
H_2 collisions.

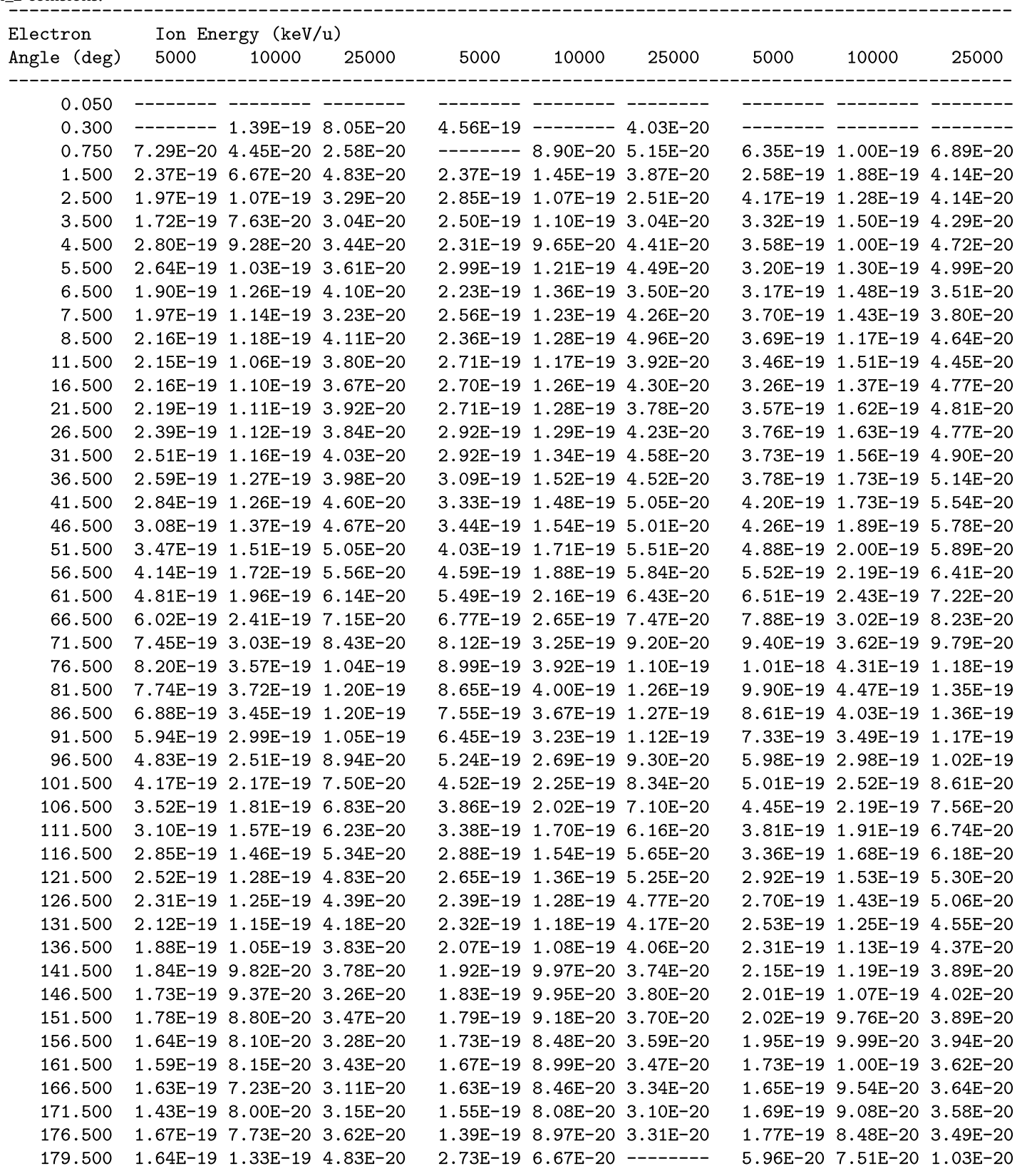


Table 22

The singly differential cross section as a function of the ejected electron angle for double ionization in $5000-25000 \mathrm{keV} / \mathrm{u} \mathrm{O}^{\wedge} 3+, 0^{\wedge} 4^{+}$, $0 \wedge 5++H_{-}$collisions.

\begin{tabular}{|c|c|c|c|c|c|c|c|c|c|}
\hline & & & & & & & & & \\
\hline 0.300 & $4.06 \mathrm{E}-19$ & $1.96 \mathrm{E}-19$ & $8.62 \mathrm{E}-20$ & $9.68 \mathrm{E}-19$ & $3.72 \mathrm{E}-19$ & 1. $21 \mathrm{E}-19$ & 1. $42 \mathrm{E}-18$ & 3. $22 \mathrm{E}-19$ & 1. $17 \mathrm{E}-19$ \\
\hline 0.750 & $6.02 E-19$ & $1.67 \mathrm{E}-19$ & 1. $10 \mathrm{E}-19$ & $6.78 \mathrm{E}-19$ & $2.98 \mathrm{E}-19$ & $4.96 \mathrm{E}-20$ & $1.08 \mathrm{E}-18$ & $3.09 E-19$ & $9.76 \mathrm{E}-20$ \\
\hline 1.500 & $4.69 \mathrm{E}-19$ & $1.80 \mathrm{E}-19$ & $3.79 \mathrm{E}-20$ & $6.78 \mathrm{E}-19$ & $3.05 \mathrm{E}-19$ & $6.20 \mathrm{E}-20$ & $1.07 \mathrm{E}-18$ & $3.44 \mathrm{E}-19$ & $8.63 \mathrm{E}-20$ \\
\hline 3.500 & $4.37 E-19$ & $1.72 \mathrm{E}-19$ & $5.76 \mathrm{E}-20$ & $6.88 \mathrm{E}-19$ & $2.71 \mathrm{E}-19$ & $6.33 E-20$ & $1.04 \mathrm{E}-18$ & 3. $16 \mathrm{E}-19$ & $7.89 \mathrm{E}-20$ \\
\hline 4.500 & $4.57 E-19$ & $1.75 \mathrm{E}-19$ & $4.60 \mathrm{E}-20$ & $6.79 E-19$ & $2.36 \mathrm{E}-19$ & $5.61 \mathrm{E}-20$ & 1. $20 \mathrm{E}-18$ & $3.37 \mathrm{E}-19$ & $7.58 \mathrm{E}-20$ \\
\hline 5.500 & $4.35 E-19$ & $1.87 \mathrm{E}-19$ & $5.74 \mathrm{E}-20$ & $7.05 \mathrm{E}-19$ & $2.58 \mathrm{E}-19$ & $6.67 \mathrm{E}-20$ & $1.07 \mathrm{E}-18$ & $3.55 \mathrm{E}-19$ & $8.82 \mathrm{E}-20$ \\
\hline 6.500 & $4.73 E-19$ & $1.86 \mathrm{E}-19$ & $6.70 \mathrm{E}-20$ & $7.03 E-19$ & $2.59 \mathrm{E}-19$ & $7.05 \mathrm{E}-20$ & $1.11 \mathrm{E}-18$ & $3.51 \mathrm{E}-19$ & $8.16 \mathrm{E}-20$ \\
\hline 7.500 & $4.57 E-19$ & $1.84 \mathrm{E}-19$ & $5.32 E-20$ & 7. $23 E-19$ & $2.47 \mathrm{E}-19$ & $6.91 \mathrm{E}-20$ & $1.09 \mathrm{E}-18$ & $3.73 E-19$ & $8.47 \mathrm{E}-20$ \\
\hline 8.500 & $4.91 \mathrm{E}-19$ & 1. $80 \mathrm{E}-19$ & $5.86 \mathrm{E}-20$ & $7.05 \mathrm{E}-19$ & $2.40 \mathrm{E}-19$ & $6.74 \mathrm{E}-20$ & $1.06 \mathrm{E}-18$ & $3.59 \mathrm{E}-19$ & $8.08 \mathrm{E}-20$ \\
\hline 26.500 & $4.94 \mathrm{E}-19$ & $1.97 \mathrm{E}-19$ & $5.35 \mathrm{E}-20$ & 7. $33 \mathrm{E}-19$ & $2.61 \mathrm{E}-19$ & $6.97 \mathrm{E}-20$ & 1. $11 \mathrm{E}-18$ & $3.75 E-19$ & $8.80 \mathrm{E}-20$ \\
\hline 31.500 & $5.08 E-19$ & $2.03 E-19$ & $5.68 \mathrm{E}-20$ & $7.48 \mathrm{E}-19$ & $2.67 \mathrm{E}-19$ & $6.97 \mathrm{E}-20$ & 1. $10 \mathrm{E}-18$ & $3.73 E-19$ & $8.85 E-20$ \\
\hline 36.500 & $5.31 E-19$ & $2.10 \mathrm{E}-19$ & $5.96 \mathrm{E}-20$ & $7.62 \mathrm{E}-19$ & $2.74 \mathrm{E}-19$ & $7.34 \mathrm{E}-20$ & 1. $14 \mathrm{E}-18$ & $3.78 \mathrm{E}-19$ & $8.92 \mathrm{E}-20$ \\
\hline 41.500 & $5.59 \mathrm{E}-19$ & $2.16 \mathrm{E}-19$ & $6.14 \mathrm{E}-20$ & $7.98 \mathrm{E}-19$ & $2.87 \mathrm{E}-19$ & $7.60 \mathrm{E}-20$ & $1.17 \mathrm{E}-18$ & $3.91 \mathrm{E}-19$ & $9.52 \mathrm{E}-20$ \\
\hline 46.500 & $5.86 E-19$ & $2.30 \mathrm{E}-19$ & $6.62 \mathrm{E}-20$ & 8. $30 \mathrm{E}-19$ & $2.99 \mathrm{E}-19$ & $7.81 \mathrm{E}-20$ & 1. $23 \mathrm{E}-18$ & $4.07 \mathrm{E}-19$ & $9.91 \mathrm{E}-20$ \\
\hline 51.500 & $6.38 \mathrm{E}-19$ & $2.44 \mathrm{E}-19$ & $6.91 \mathrm{E}-20$ & $8.91 \mathrm{E}-19$ & $3.18 \mathrm{E}-19$ & $8.38 \mathrm{E}-20$ & 1. $29 \mathrm{E}-18$ & $4.25 \mathrm{E}-19$ & $1.02 \mathrm{E}-19$ \\
\hline 56.500 & $7.09 E-19$ & $2.67 \mathrm{E}-19$ & $7.56 \mathrm{E}-20$ & $9.68 \mathrm{E}-19$ & $3.45 \mathrm{E}-19$ & $8.80 \mathrm{E}-20$ & 1. $37 \mathrm{E}-18$ & $4.53 E-19$ & $1.08 \mathrm{E}-19$ \\
\hline 61.500 & $8.20 E-19$ & $3.00 \mathrm{E}-19$ & $8.05 E-20$ & 1. $10 \mathrm{E}-18$ & $3.77 \mathrm{E}-19$ & $9.40 \mathrm{E}-20$ & $1.51 \mathrm{E}-18$ & $5.05 E-19$ & 1. $14 \mathrm{E}-19$ \\
\hline 66.500 & $9.79 \mathrm{E}-19$ & $3.48 \mathrm{E}-19$ & $9.21 \mathrm{E}-20$ & 1. $28 \mathrm{E}-18$ & $4.37 \mathrm{E}-19$ & $1.08 \mathrm{E}-19$ & 1. $73 \mathrm{E}-18$ & $5.61 \mathrm{E}-19$ & 1. $29 \mathrm{E}-19$ \\
\hline 71.500 & 1. $14 \mathrm{E}-18$ & $4.26 \mathrm{E}-19$ & 1. $10 \mathrm{E}-19$ & $1.49 \mathrm{E}-18$ & $5.27 \mathrm{E}-19$ & 1. $24 \mathrm{E}-19$ & 1. $95 \mathrm{E}-18$ & $6.59 \mathrm{E}-19$ & $1.45 \mathrm{E}-19$ \\
\hline 76.500 & 1. $23 \mathrm{E}-18$ & $4.95 \mathrm{E}-19$ & 1. $32 \mathrm{E}-19$ & $1.57 \mathrm{E}-18$ & $6.02 \mathrm{E}-19$ & $1.52 \mathrm{E}-19$ & $2.07 \mathrm{E}-18$ & $7.54 \mathrm{E}-19$ & $1.76 \mathrm{E}-19$ \\
\hline 116.500 & $3.89 E-19$ & $1.94 \mathrm{E}-19$ & $6.43 \mathrm{E}-20$ & $4.94 \mathrm{E}-19$ & $2.35 \mathrm{E}-19$ & $7.47 \mathrm{E}-20$ & $6.60 \mathrm{E}-19$ & $2.93 E-19$ & $8.76 \mathrm{E}-20$ \\
\hline 121.500 & $3.45 \mathrm{E}-19$ & 1. $75 \mathrm{E}-19$ & $6.01 \mathrm{E}-20$ & $4.46 \mathrm{E}-19$ & $2.12 \mathrm{E}-19$ & $6.87 \mathrm{E}-20$ & $5.79 \mathrm{E}-19$ & $2.65 \mathrm{E}-19$ & $8.01 \mathrm{E}-20$ \\
\hline 126.500 & 3. $22 \mathrm{E}-19$ & $1.60 \mathrm{E}-19$ & $5.40 E-20$ & $4.01 \mathrm{E}-19$ & $1.94 \mathrm{E}-19$ & $6.28 \mathrm{E}-20$ & $5.16 \mathrm{E}-19$ & $2.36 \mathrm{E}-19$ & $7.35 \mathrm{E}-20$ \\
\hline 131.500 & $2.87 E-19$ & $1.48 \mathrm{E}-19$ & $5.23 E-20$ & $3.65 \mathrm{E}-19$ & $1.77 \mathrm{E}-19$ & $5.87 \mathrm{E}-20$ & $4.74 \mathrm{E}-19$ & $2.21 \mathrm{E}-19$ & $6.83 E-20$ \\
\hline 136.500 & $2.75 E-19$ & 1. $36 \mathrm{E}-19$ & $4.80 E-20$ & $3.40 \mathrm{E}-19$ & 1. $66 \mathrm{E}-19$ & $5.49 \mathrm{E}-20$ & $4.39 \mathrm{E}-19$ & $2.01 \mathrm{E}-19$ & $6.38 \mathrm{E}-20$ \\
\hline 141.500 & $2.47 \mathrm{E}-19$ & 1. $25 \mathrm{E}-19$ & $4.55 \mathrm{E}-20$ & $3.16 \mathrm{E}-19$ & $1.55 \mathrm{E}-19$ & $5.32 \mathrm{E}-20$ & $4.04 \mathrm{E}-19$ & 1. $93 \mathrm{E}-19$ & $5.88 \mathrm{E}-20$ \\
\hline 146.500 & $2.38 E-19$ & 1. $23 \mathrm{E}-19$ & $4.37 \mathrm{E}-20$ & $3.00 \mathrm{E}-19$ & 1. $48 \mathrm{E}-19$ & $4.90 \mathrm{E}-20$ & $3.89 E-19$ & $1.77 \mathrm{E}-19$ & $5.89 \mathrm{E}-20$ \\
\hline 151.500 & $2.28 E-19$ & 1. $14 \mathrm{E}-19$ & $4.27 \mathrm{E}-20$ & $2.78 \mathrm{E}-19$ & $1.40 \mathrm{E}-19$ & $4.73 E-20$ & $3.68 \mathrm{E}-19$ & 1. $72 \mathrm{E}-19$ & $5.58 \mathrm{E}-20$ \\
\hline 156.500 & $2.17 \mathrm{E}-19$ & $1.09 \mathrm{E}-19$ & $4.26 \mathrm{E}-20$ & $2.72 \mathrm{E}-19$ & $1.33 \mathrm{E}-19$ & $4.40 \mathrm{E}-20$ & $3.56 \mathrm{E}-19$ & $1.68 \mathrm{E}-19$ & $5.44 \mathrm{E}-20$ \\
\hline 161.500 & $2.13 E-19$ & 1. $11 \mathrm{E}-19$ & $3.85 \mathrm{E}-20$ & $2.67 \mathrm{E}-19$ & 1. $30 \mathrm{E}-19$ & $4.51 \mathrm{E}-20$ & $3.48 \mathrm{E}-19$ & 1. $63 \mathrm{E}-19$ & $5.12 \mathrm{E}-20$ \\
\hline 166.500 & $2.07 E-19$ & $1.07 \mathrm{E}-19$ & $3.80 E-20$ & $2.54 \mathrm{E}-19$ & 1. $30 \mathrm{E}-19$ & $4.42 \mathrm{E}-20$ & $3.16 \mathrm{E}-19$ & $1.55 \mathrm{E}-19$ & $4.84 \mathrm{E}-20$ \\
\hline 171.500 & $2.12 \mathrm{E}-19$ & $1.04 \mathrm{E}-19$ & $3.94 \mathrm{E}-20$ & $2.44 \mathrm{E}-19$ & $1.31 \mathrm{E}-19$ & $4.30 \mathrm{E}-20$ & $3.08 \mathrm{E}-19$ & $1.55 \mathrm{E}-19$ & $4.92 \mathrm{E}-20$ \\
\hline 176.500 & $2.03 E-19$ & $1.06 \mathrm{E}-19$ & $3.58 \mathrm{E}-20$ & $2.43 E-19$ & 1. $25 \mathrm{E}-19$ & $4.09 E-20$ & 3. $22 \mathrm{E}-19$ & $1.63 \mathrm{E}-19$ & $5.29 \mathrm{E}-20$ \\
\hline 179.500 & $1.77 \mathrm{E}-19$ & $1.10 \mathrm{E}-19$ & $3.10 \mathrm{E}-20$ & $3.65 \mathrm{E}-19$ & 1. $27 \mathrm{E}-19$ & $3.72 \mathrm{E}-20$ & $2.92 \mathrm{E}-19$ & 1. $35 \mathrm{E}-19$ & $3.38 \mathrm{E}-20$ \\
\hline
\end{tabular}


Table 23

The singly differential cross section as a function of the ejected electron angle for double ionization in $5000-25000 \mathrm{keV} / \mathrm{u} \mathrm{O}^{\wedge} 6+$, $0^{\wedge} 7+$, $\mathrm{O}^{\wedge} 8++\mathrm{H}_{-}$collisions.

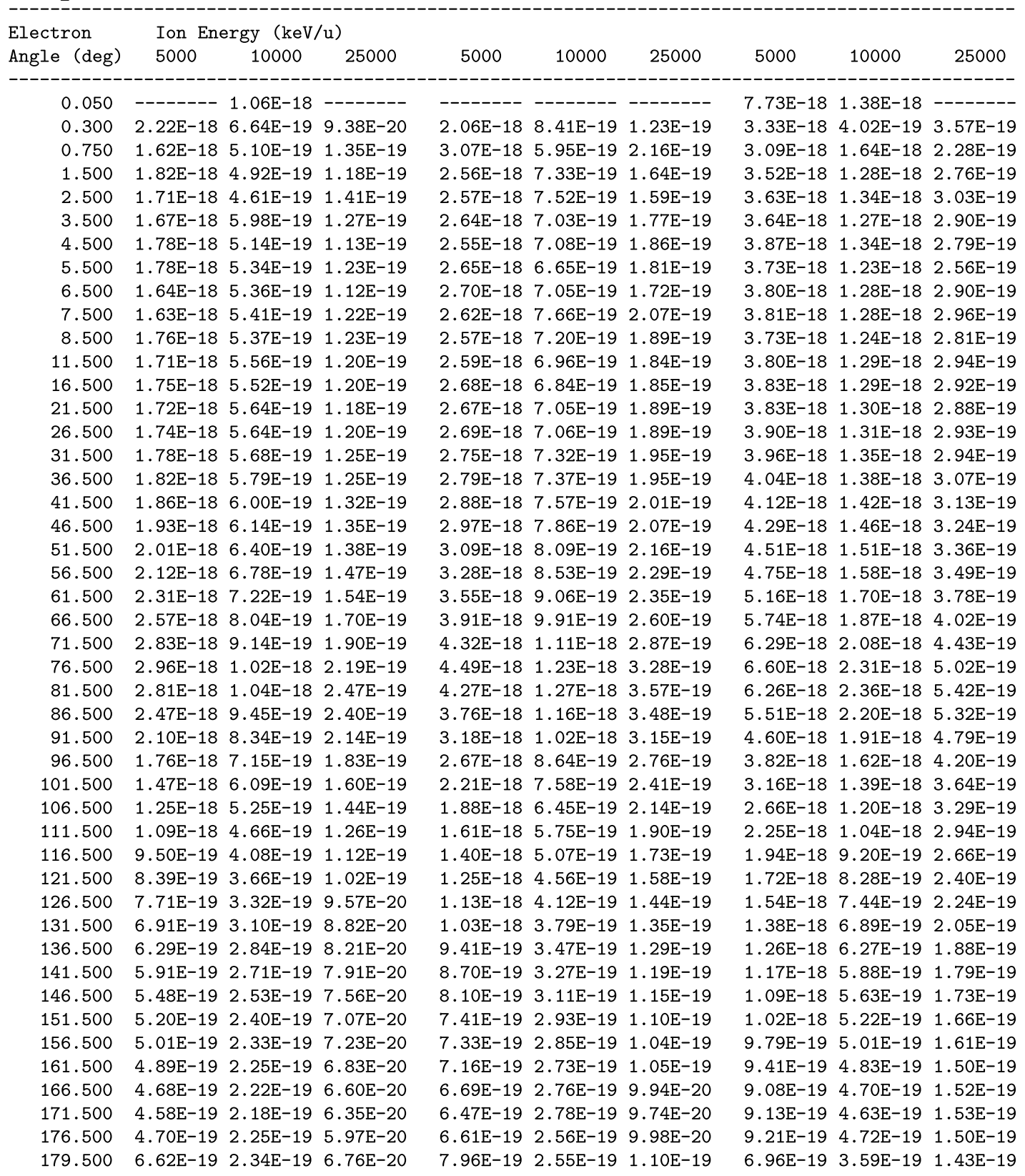


Table 24

The singly differential cross section as a function of the ejected electron energy for transfer ionization in $5000-25000 \mathrm{keV} / \mathrm{u} \mathrm{O}, 0^{\wedge}+, 0^{\wedge} 2+$ $+\mathrm{H} \_2$ collisions.

\begin{tabular}{|c|c|c|c|c|c|c|c|c|c|}
\hline \multirow{3}{*}{$\begin{array}{l}\text { Electron } \\
\text { Energy (eV) }\end{array}$} & \multicolumn{9}{|c|}{ Ion Energy $(\mathrm{keV} / \mathrm{u})$} \\
\hline & 5000 & 10000 & 25000 & 5000 & 10000 & 25000 & 5000 & 10000 & 25000 \\
\hline & & & & & & & & & \\
\hline 0.500 & -------- & -------- & -------- & -------- & -------- & -------- & $1.10 \mathrm{E}-24$ & -------- & -------- \\
\hline 5.500 & ------- & ------- & -------- & $2.35 E-24$ & ------- & ------- & $2.57 \mathrm{E}-24$ & $1.95 \mathrm{E}-25$ & ------- \\
\hline 30.000 & ------- & ------- & ------- & $8.44 \mathrm{E}-25$ & $8.25 E-26$ & ------- & $9.35 E-25$ & $2.20 \mathrm{E}-26$ & ------ \\
\hline 75.000 & -------- & -------- & -------- & $1.69 \mathrm{E}-25$ & $2.20 \mathrm{E}-26$ & -------- & $2.86 \mathrm{E}-25$ & $3.52 \mathrm{E}-26$ & $1.47 \mathrm{E}-26$ \\
\hline 300.000 & ------- & ------- & ------- & $2.11 \mathrm{E}-26$ & ------- & ------- & $4.40 E-26$ & $4.40 \mathrm{E}-27$ & ------ \\
\hline 750.000 & ------- & ------- & -------- & -------- & ------- & -------- & $6.60 \mathrm{E}-27$ & ------- & ------- \\
\hline 3000.000 & -------- & -------- & -------- & $1.06 \mathrm{E}-27$ & -------- & -------- & -------- & -------- & -- \\
\hline
\end{tabular}

Table 25

The singly differential cross section as a function of the ejected electron energy for transfer ionization in $5000-25000 \mathrm{keV} / \mathrm{u} \mathrm{O`3+,} 0^{\wedge} 4^{+}$, 0^5+ + H_2 collisions.

\begin{tabular}{|c|c|c|c|c|c|c|c|c|c|}
\hline \multirow{3}{*}{$\begin{array}{l}\text { Electron } \\
\text { Energy (eV) }\end{array}$} & \multicolumn{9}{|c|}{ Ion Energy $(\mathrm{keV} / \mathrm{u})$} \\
\hline & 5000 & 10000 & 25000 & 5000 & 10000 & 25000 & 5000 & 10000 & 25000 \\
\hline & & & & & & & & & \\
\hline 0.500 & $1.94 \mathrm{E}-24$ & ------- & ------ & $4.50 E-24$ & $4.40 E-25$ & ------- & $1.76 \mathrm{E}-23$ & $5.86 \mathrm{E}-25$ & ------- \\
\hline 5.500 & $3.02 \mathrm{E}-24$ & $3.45 E-25$ & ------ & $2.17 \mathrm{E}-24$ & $2.93 E-25$ & ------- & $1.02 E-23$ & $3.91 \mathrm{E}-25$ & ------ \\
\hline 30.000 & $1.31 \mathrm{E}-24$ & -------- & ------- & $7.88 \mathrm{E}-25$ & $8.80 E-26$ & $4.40 \mathrm{E}-28$ & $2.37 \mathrm{E}-24$ & $7.33 E-26$ & ------- \\
\hline 75.000 & $1.94 \mathrm{E}-25$ & -------- & ------- & $3.00 E-25$ & $3.52 E-26$ & -------- & $7.04 \mathrm{E}-25$ & $3.52 \mathrm{E}-26$ & ------- \\
\hline 300.000 & $8.73 E-26$ & ------- & ------- & $4.13 E-26$ & 1. $21 \mathrm{E}-26$ & -------- & $9.67 \mathrm{E}-26$ & $5.86 \mathrm{E}-27$ & --- \\
\hline 750.000 & ------- & -------- & ------- & 1. $20 \mathrm{E}-26$ & ------- & -------- & $7.04 \mathrm{E}-27$ & ------- & ------ \\
\hline 3000.000 & $1.94 \mathrm{E}-27$ & $3.88 E-28$ & ------- & $3.75 E-28$ & $1.10 \mathrm{E}-28$ & ------- & $2.64 \mathrm{E}-27$ & ------- & ------- \\
\hline
\end{tabular}

Table 26

The singly differential cross section as a function of the ejected electron energy for transfer ionization in 5000-25000 keV/u 0^6+, 0^7+, $\mathrm{O}^{\wedge} 8++\mathrm{H} \_2$ collisions.

\begin{tabular}{|c|c|c|c|c|c|c|c|c|c|}
\hline \multirow{2}{*}{$\begin{array}{l}\text { Electron } \\
\text { Energy (eV) }\end{array}$} & \multicolumn{9}{|c|}{ Ion Energy $(\mathrm{keV} / \mathrm{u})$} \\
\hline & 5000 & 10000 & 25000 & 5000 & 10000 & 25000 & 5000 & 10000 & 25000 \\
\hline--- & & & & & & & & & \\
\hline 0.500 & $2.40 \mathrm{E}-23$ & $4.40 E-25$ & ------- & $1.84 \mathrm{E}-23$ & $3.88 \mathrm{E}-24$ & ------- & $2.70 E-23$ & $8.80 \mathrm{E}-25$ & ------- \\
\hline 5.500 & $1.53 \mathrm{E}-23$ & $3.42 E-25$ & ------- & $2.25 E-23$ & $1.51 \mathrm{E}-24$ & ------- & $3.49 E-23$ & 1. $27 E-24$ & \\
\hline 30.000 & $4.65 \mathrm{E}-24$ & $1.54 \mathrm{E}-25$ & $1.08 \mathrm{E}-26$ & $8.50 E-24$ & $1.45 \mathrm{E}-25$ & ------- & $9.29 E-24$ & $4.51 \mathrm{E}-25$ & ------- \\
\hline 75.000 & $9.61 \mathrm{E}-25$ & $2.64 \mathrm{E}-26$ & -------- & $1.65 \mathrm{E}-24$ & $7.76 \mathrm{E}-26$ & -------- & $2.50 \mathrm{E}-24$ & $1.67 \mathrm{E}-25$ & --- \\
\hline 300.000 & $9.01 \mathrm{E}-26$ & $5.50 \mathrm{E}-27$ & -------- & $2.41 \mathrm{E}-25$ & ------- & -------- & $3.38 \mathrm{E}-25$ & $8.80 E-27$ & ------- \\
\hline 750.000 & 1. $20 \mathrm{E}-26$ & $3.52 \mathrm{E}-27$ & ------- & $2.76 \mathrm{E}-26$ & ------- & -------- & $4.73 E-26$ & -------- & --- \\
\hline 3000.000 & ------ & ------- & ------- & $4.60 \mathrm{E}-27$ & ------ & ------- & 4. $22 \mathrm{E}-27$ & ------- & ------ \\
\hline
\end{tabular}


Table 27

The singly differential cross section as a function of the ejected electron angle for transfer ionization in $5000-25000 \mathrm{keV} / \mathrm{u} \mathrm{O}^{\circ} \mathrm{O}^{\wedge}+, \mathrm{O}^{\wedge} 2+$ $+\mathrm{H} \_2$ collisions.

\begin{tabular}{|c|c|c|c|c|c|c|c|c|c|}
\hline \multirow{3}{*}{$\begin{array}{l}\text { Electron } \\
\text { Angle (deg) }\end{array}$} & \multicolumn{9}{|c|}{ Ion Energy $(\mathrm{keV} / \mathrm{u})$} \\
\hline & 5000 & 10000 & 25000 & 5000 & 10000 & 25000 & 5000 & 10000 & 25000 \\
\hline & & & & & & & & & \\
\hline 10.000 & -------- & -------- & -------- & -------- & ------- & -------- & -------- & -------- & \\
\hline 30.000 & -------- & -------- & ------- & -------- & ------ & -------- & $9.02 E-24$ & -------- & ----- \\
\hline 50.000 & -------- & -------- & -------- & $8.79 E-24$ & ------- & -------- & $6.54 \mathrm{E}-24$ & -------- & \\
\hline 70.000 & -------- & -------- & -------- & $9.22 \mathrm{E}-24$ & ------- & -------- & $1.07 \mathrm{E}-23$ & -------- & \\
\hline 90.000 & ------- & ------- & ------- & $4.81 E-24$ & ----- & ------- & $9.52 E-24$ & ------- & ------ \\
\hline 110.000 & ------- & ------- & ------- & $7.17 E-24$ & ------ & ------- & $5.33 E-24$ & ------- & ---- \\
\hline 130.000 & -------- & ------- & ------- & ------- & ------- & -------- & $5.23 E-24$ & -------- & ----- \\
\hline 150.000 & -------- & -------- & -------- & -------- & ------- & -------- & $9.02 E-24$ & -------- & ---- \\
\hline 170.000 & -------- & ---- & -------- & -------- & ------ & -------- & -------- & ---- & \\
\hline
\end{tabular}

\section{Table 28}

The singly differential cross section as a function of the ejected electron angle for transfer ionization in $5000-25000 \mathrm{keV} / \mathrm{u} \mathrm{O}^{\wedge} 3+, \mathrm{O}^{\wedge} 4+$, O^5++ H_2 collisions.

\begin{tabular}{|c|c|c|c|c|c|c|c|c|c|}
\hline \multirow{2}{*}{$\begin{array}{l}\text { Electron } \\
\text { Angle (deg) }\end{array}$} & \multicolumn{9}{|c|}{ Ion Energy $(\mathrm{keV} / \mathrm{u})$} \\
\hline & 5000 & 10000 & 25000 & 5000 & 10000 & 25000 & 5000 & 10000 & 25000 \\
\hline & & & & & & & & & \\
\hline 10.000 & ---- & --- & -------- & -------- & --- & -------- & -------- & -------- & \\
\hline 30.000 & -------- & -------- & -------- & $9.58 \mathrm{E}-24$ & ------- & -------- & 1. $92 \mathrm{E}-23$ & $5.35 \mathrm{E}-25$ & \\
\hline 50.000 & 1. $15 \mathrm{E}-23$ & $9.23 E-25$ & ------- & $7.15 \mathrm{E}-24$ & ------- & -------- & $3.77 \mathrm{E}-23$ & $1.40 \mathrm{E}-24$ & \\
\hline 70.000 & 1. $69 \mathrm{E}-23$ & $7.53 E-25$ & ------- & 1. $38 \mathrm{E}-23$ & $1.71 \mathrm{E}-24$ & -------- & 3. $24 \mathrm{E}-23$ & $8.54 \mathrm{E}-25$ & \\
\hline 90.000 & $1.41 \mathrm{E}-23$ & $7.07 \mathrm{E}-25$ & -------- & $7.53 \mathrm{E}-24$ & $2.01 \mathrm{E}-24$ & -------- & $3.05 E-23$ & $1.07 \mathrm{E}-24$ & \\
\hline 110.000 & 1. $32 \mathrm{E}-23$ & ------ & ------ & $4.37 \mathrm{E}-24$ & ------- & $8.54 \mathrm{E}-27$ & $1.54 \mathrm{E}-23$ & $5.69 \mathrm{E}-25$ & \\
\hline 130.000 & ------- & ------ & ------ & $6.25 E-24$ & ------ & ------- & $1.05 E-23$ & $1.05 \mathrm{E}-24$ & -- \\
\hline 150.000 & -------- & ------- & ------- & -------- & ------- & -------- & -------- & $5.35 \mathrm{E}-25$ & -- \\
\hline 170.000 & -------- & -------- & -------- & -------- & -------- & -------- & -------- & $1.54 \mathrm{E}-24$ & \\
\hline
\end{tabular}

Table 29

The singly differential cross section as a function of the ejected electron angle for transfer ionization in $5000-25000 \mathrm{keV} / \mathrm{u} \mathrm{O}^{\wedge} 6+, \mathrm{O}^{\wedge} 7+$, $0 \wedge 8++$ H_2 collisions.

\begin{tabular}{|c|c|c|c|c|c|c|c|c|c|}
\hline \multirow{3}{*}{$\begin{array}{l}\text { Electron } \\
\text { Angle (deg) }\end{array}$} & \multicolumn{9}{|c|}{ Ion Energy $(\mathrm{keV} / \mathrm{u})$} \\
\hline & 5000 & 10000 & 25000 & 5000 & 10000 & 25000 & 5000 & 10000 & 25000 \\
\hline & & & & & & & & & - \\
\hline 10.000 & -------- & ------- & ------ & 1. $21 \mathrm{E}-22$ & ------ & ------- & $1.51 \mathrm{E}-22$ & $1.04 \mathrm{E}-23$ & -- \\
\hline 30.000 & $4.38 \mathrm{E}-23$ & $2.41 \mathrm{E}-24$ & ------ & $3.35 E-23$ & ------ & -------- & $8.62 E-23$ & $5.61 \mathrm{E}-24$ & \\
\hline 50.000 & $6.43 E-23$ & -------- & ------- & $6.84 \mathrm{E}-23$ & $2.31 \mathrm{E}-24$ & -------- & $9.05 E-23$ & $2.88 \mathrm{E}-24$ & \\
\hline 70.000 & $5.54 \mathrm{E}-23$ & ------- & $1.05 \mathrm{E}-25$ & $1.00 E-22$ & $1.88 \mathrm{E}-24$ & -------- & $1.31 \mathrm{E}-22$ & $3.84 \mathrm{E}-24$ & \\
\hline 90.000 & $5.20 \mathrm{E}-23$ & $2.41 \mathrm{E}-24$ & $9.82 \mathrm{E}-26$ & $9.85 \mathrm{E}-23$ & $5.31 \mathrm{E}-24$ & -------- & $8.62 \mathrm{E}-23$ & $3.61 \mathrm{E}-24$ & \\
\hline 110.000 & -------- & -------- & ------- & $3.35 E-23$ & $9.41 \mathrm{E}-25$ & -------- & $6.23 E-23$ & $2.99 \mathrm{E}-24$ & \\
\hline 130.000 & ------- & ------ & ------ & $2.74 \mathrm{E}-23$ & 1. $15 \mathrm{E}-24$ & ------- & $5.23 E-23$ & $2.62 \mathrm{E}-24$ & -- \\
\hline 150.000 & -------- & -------- & ------ & $2.93 E-23$ & $1.77 \mathrm{E}-24$ & -------- & $1.85 \mathrm{E}-23$ & -------- & \\
\hline 170.000 & -------- & -------- & ------- & -------- & $5.09 \mathrm{E}-24$ & -------- & -------- & -------- & \\
\hline
\end{tabular}


Table 30

The singly differential cross section as a function of the ejected electron energy for single stripping in 5000-25000 keV/u 0, $0^{\wedge}+, 0^{\wedge} 2++$ H_2 collisions.

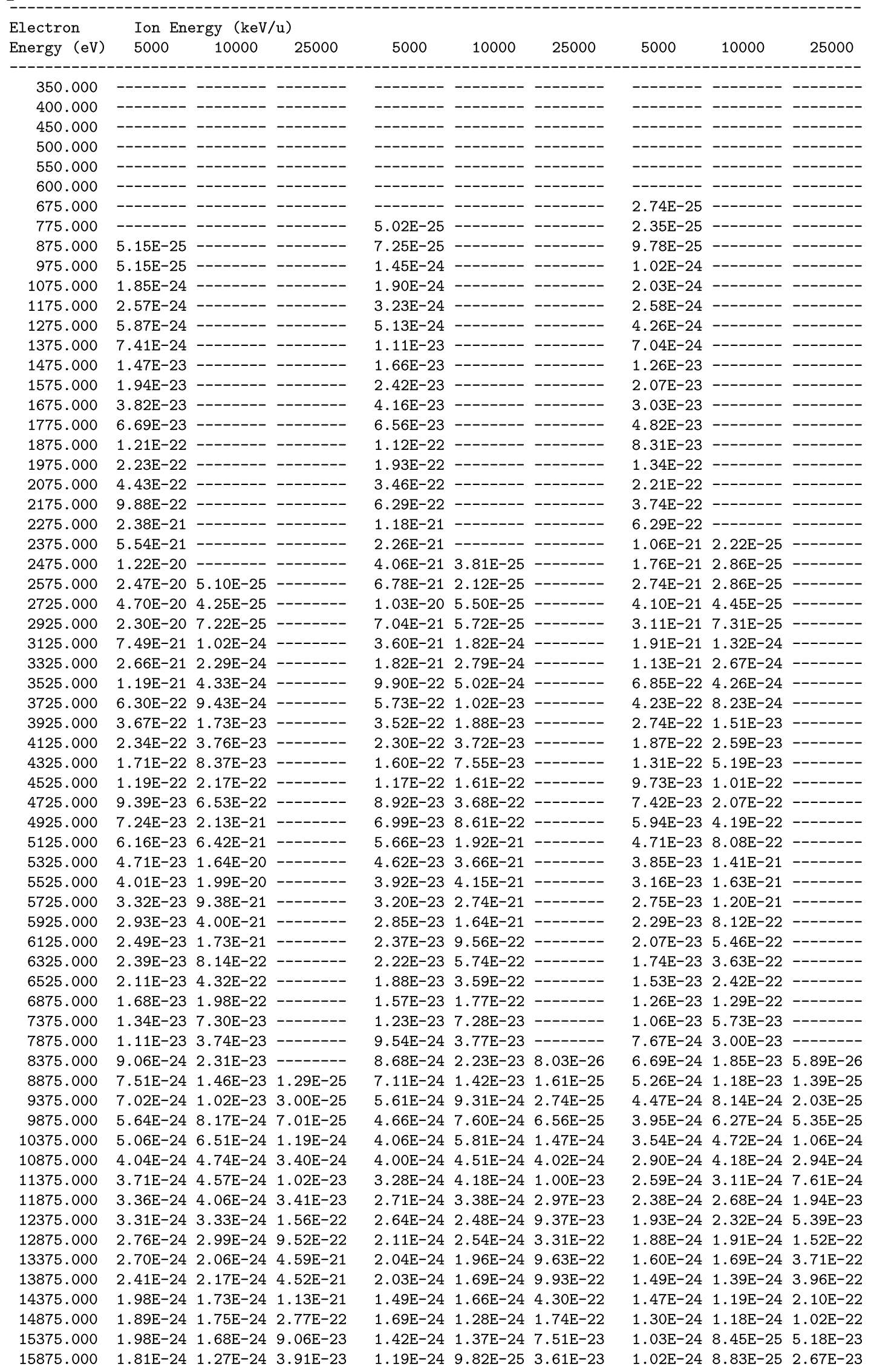


Table 30 (continued)

$16375.000 \quad 1.48 \mathrm{E}-24 \quad 1.31 \mathrm{E}-24 \quad 1.95 \mathrm{E}-23$ $17125.000 \quad 1.12 \mathrm{E}-24 \quad 1.10 \mathrm{E}-24 \quad 9.24 \mathrm{E}-24$ $18125.000 \quad 9.99 \mathrm{E}-25 \quad 9.51 \mathrm{E}-25 \quad 4.20 \mathrm{E}-24$ $19125.000 \quad 9.26 \mathrm{E}-25 \quad 8.15 \mathrm{E}-25 \quad 2.49 \mathrm{E}-24$ $20125.000 \quad 8.54 \mathrm{E}-25 \quad 5.78 \mathrm{E}-25 \quad 1.76 \mathrm{E}-24$ $21125.000 \quad 8.34 \mathrm{E}-25 \quad 6.63 \mathrm{E}-25 \quad 1.05 \mathrm{E}-24$ $22125.000 \quad 7.41 \mathrm{E}-25 \quad 4.50 \mathrm{E}-25 \quad 9.44 \mathrm{E}-25$ $23125.000 \quad 6.28 \mathrm{E}-25 \quad 5.10 \mathrm{E}-25 \quad 7.72 \mathrm{E}-25$ $24125.000 \quad 2.99 \mathrm{E}-25 \quad 3.65 \mathrm{E}-25 \quad 6.22 \mathrm{E}-25$ $25125.000 \quad 1.03 \mathrm{E}-25 \quad 3.65 \mathrm{E}-25 \quad 5.36 \mathrm{E}-25$ $26125.000-----2.97 \mathrm{E}-25 \quad 3.29 \mathrm{E}-25$ 27125.000 ------ $2.38 \mathrm{E}-25$ 3.00E-25 28125.000 ------ 2.46E-25 2.57E-25 29125.000 ------- 2.63E-25 2.22E-25 30125.000 ------ 2.29E-25 $1.36 \mathrm{E}-25$ 31125.000 ----- 2.72E-25 $1.86 \mathrm{E}-25$ 32125.000 ------ $1.87 \mathrm{E}-251.72 \mathrm{E}-25$ 33125.000 ------ $1.19 \mathrm{E}-251.79 \mathrm{E}-25$ 34125.000 ------ $1.87 \mathrm{E}-25 \quad 1.29 \mathrm{E}-25$ 35125.000 ------ $1.44 \mathrm{E}-25 \quad 1.50 \mathrm{E}-25$ 36125.000 ------- $1.53 \mathrm{E}-25 \quad 1.29 \mathrm{E}-25$ 37875.000 ------ $1.29 \mathrm{E}-251.17 \mathrm{E}-25$ 40375.000 ----- $1.05 \mathrm{E}-25 \quad 5.72 \mathrm{E}-26$ 42875.000 ------ $1.09 \mathrm{E}-25 \quad 6.86 \mathrm{E}-26$ 45375.000 ------ $1.02 \mathrm{E}-25 \quad 4.29 \mathrm{E}-26$ $47875.000 \quad-----3.74 \mathrm{E}-26 \quad 4.58 \mathrm{E}-26$ 50375.000 ------ ------ 3.72E-26 52875.000 ------ ------ 3.72E-26 55375.000 - - - - - - - 3.72E-26 57875.000 ------ ------ 4.00E-26 60375.000 ------- ------- 2.29E-26 62875.000 --65375.000 ------- ------- 2.00E-26 67875.000 ------- ------- 2.29E-26

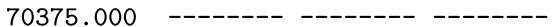
72875.000
1.32E-24 1.10E-24 1.93E-23 1.19E-24 8.55E-25 9.22E-24 $9.98 \mathrm{E}-25 \quad 7.70 \mathrm{E}-25 \quad 4.24 \mathrm{E}-24$ 8.19E-25 6.69E-25 2.34E-24 8.14E-25 4.83E-25 $1.41 \mathrm{E}-24$ $6.35 \mathrm{E}-25 \quad 4.45 \mathrm{E}-25 \quad 1.03 \mathrm{E}-24$ 4.79E-25 3.43E-25 7.59E-25 $4.96 \mathrm{E}-25 \quad 3.68 \mathrm{E}-25 \quad 6.15 \mathrm{E}-25$ $2.68 \mathrm{E}-25 \quad 3.81 \mathrm{E}-25 \quad 5.02 \mathrm{E}-25$ $6.69 \mathrm{E}-26 \quad 4.11 \mathrm{E}-25 \quad 4.48 \mathrm{E}-25$ $2.79 \mathrm{E}-26 \quad 2.58 \mathrm{E}-25 \quad 3.21 \mathrm{E}-25$ ------- 2.41E-25 2.64E-25 ------ 2.71E-25 2.11E-25 ------- 2.46E-25 2.31E-25 ------ 2.16E-25 $1.67 \mathrm{E}-25$ ------ 1.52E-25 1.54E-25 ------ 1.40E-25 1.24E-25 ------ 1.69E-25 1.10E-25 ------ 1.23E-25 1.34E-25 ------ 1.02E-25 1.30E-25 ------ $1.95 \mathrm{E}-25 \quad 1.04 \mathrm{E}-25$ ------ 1.19E-25 8.29E-26 ------ 9.82E-26 5.62E-26 ------- 9.14E-26 6.02E-26 ----- 5.08E-26 5.49E-26 ------ 3.05E-26 5.49E-26 ------ 1.19E-26 4.15E-26 ------- ------- 2.54E-26 ------ ------ $2.81 \mathrm{E}-26$ ------ ------ 2.68E-26 ------ ------ $1.61 \mathrm{E}-26$ ------- ------- 2.14E-26 ------- ------- $1.87 \mathrm{E}-26$ ------ ------ $2.68 \mathrm{E}-26$ ------ ------ $1.87 \mathrm{E}-26$ ------- ------- $1.61 \mathrm{E}-26$
1.03E-24 8.13E-25 1.50E-23 9.89E-25 $7.40 \mathrm{E}-25 \quad 7.41 \mathrm{E}-24$ $7.90 \mathrm{E}-25 \quad 5.37 \mathrm{E}-25 \quad 3.30 \mathrm{E}-24$ $6.14 \mathrm{E}-25 \quad 5.02 \mathrm{E}-25 \quad 1.91 \mathrm{E}-24$ $6.69 \mathrm{E}-25 \quad 4.89 \mathrm{E}-25 \quad 1.30 \mathrm{E}-24$ 6. 34E-25 3.91E-25 9.23E-25 $3.79 \mathrm{E}-25 \quad 3.02 \mathrm{E}-25 \quad 6.15 \mathrm{E}-25$ $4.65 \mathrm{E}-25 \quad 2.92 \mathrm{E}-25 \quad 4.87 \mathrm{E}-25$ $2.27 \mathrm{E}-25 \quad 2.54 \mathrm{E}-25 \quad 4.17 \mathrm{E}-25$ $\begin{array}{lll}7.82 \mathrm{E}-26 & 2.38 \mathrm{E}-25 & 3.02 \mathrm{E}-25\end{array}$ $2.74 \mathrm{E}-26 \quad 2.16 \mathrm{E}-25 \quad 2.84 \mathrm{E}-25$ ------ 2.41E-25 2.22E-25 ------ 2.48E-25 $1.85 \mathrm{E}-25$ ------- $1.62 \mathrm{E}-25 \quad 1.93 \mathrm{E}-25$ ----- 2.00E-25 $1.18 \mathrm{E}-25$ ------ 1.43E-25 $1.31 \mathrm{E}-25$ ----- $1.59 \mathrm{E}-25$ 1.10E-25 ------ 9.53E-26 9.63E-26 ------ $1.27 \mathrm{E}-25$ 8.56E-26 ------ 9.85E-26 8.29E-26 ----- 9.85E-26 8.03E-26 $------9.02 \mathrm{E}-26 \quad 7.81 \mathrm{E}-26$ ------ 6.86E-26 5.67E-26 ------ 5.34E-26 5.67E-26 ------ 5.21E-26 4.07E-26 ------ $4.19 \mathrm{E}-26 \quad 3.32 \mathrm{E}-26$ ------ 7.63E-27 2.35E-26 ------ ------ $1.71 \mathrm{E}-26$ ------- ------ 2.35E-26 ------ ------ $1.61 \mathrm{E}-26$ ------- ------- $1.28 \mathrm{E}-26$ ------ ------ $1.28 \mathrm{E}-26$ ------ ------ 2.03E-26 ------- ------- $1.50 \mathrm{E}-26$ ------- ------- 1.61E-26 ----- ----- $1.07 \mathrm{E}-26$ 
Table 31

The singly differential cross section as a function of the ejected electron energy for single stripping in $5000-25000 \mathrm{keV} / \mathrm{u} \mathrm{O`3+}, 0^{\wedge} 4+, 0^{\wedge} 5^{+}$ $+\mathrm{H} \_2$ collisions.

\begin{tabular}{|c|c|c|c|c|c|c|c|c|c|}
\hline $\begin{array}{l}\text { Electron } \\
\text { Energy (eV) }\end{array}$ & & & & & & & & & \\
\hline 400.000 & ------- & --- & --- & ------- & & & ------- & & \\
\hline 450.000 & ------- & & ------- & ------- & & --- & -------- & & \\
\hline 500.000 & ------- & --- & ------- & ------- & --- & --- & ------- & --- & -- \\
\hline 600.000 & -------- & & -------- & -------- & & ------- & ------- & ------- & \\
\hline 675.000 & $1.73 E-25$ & -------- & -------- & -------- & --- & -------- & -------- & --- & -- \\
\hline 775.000 & $4.50 \mathrm{E}-25$ & ---- & -------- & $1.53 \mathrm{E}-25$ & ----- & ----- & $1.78 \mathrm{E}-25$ & & \\
\hline 875.000 & $6.24 \mathrm{E}-25$ & & -------- & $3.50 \mathrm{E}-25$ & & -------- & $2.31 \mathrm{E}-25$ & & \\
\hline 975.000 & $9.35 E-25$ & -------- & -------- & $5.47 E-25$ & ------- & -------- & $2.66 \mathrm{E}-25$ & ------ & ------- \\
\hline 1075.000 & 1. $35 \mathrm{E}-24$ & --- & -------- & $7.88 \mathrm{E}-25$ & ----- & ------ & $5.86 \mathrm{E}-25$ & & \\
\hline 1475.000 & $7.69 \mathrm{E}-24$ & & ----- & $5.43 E-24$ & & & 2. $22 \mathrm{E}-24$ & & \\
\hline 1575.000 & 1. $34 \mathrm{E}-23$ & -- & ------- & $7.99 E-24$ & ------- & ------- & 3. $21 \mathrm{E}-24$ & & \\
\hline 1675.000 & $2.06 \mathrm{E}-23$ & -- & -------- & 1. $20 \mathrm{E}-23$ & -- & --- & $5.04 \mathrm{E}-24$ & & \\
\hline 1775.000 & 3. $29 \mathrm{E}-23$ & -- & ----- & $1.87 \mathrm{E}-23$ & -- & & $8.12 E-24$ & & \\
\hline 1875.000 & $5.17 \mathrm{E}-23$ & --- & ------- & $2.91 \mathrm{E}-23$ & ------- & ------- & $1.16 \mathrm{E}-23$ & & \\
\hline 1975.000 & $8.27 \mathrm{E}-23$ & -- & ------ & 4. $33 \mathrm{E}-23$ & ------- & & $1.70 \mathrm{E}-23$ & & \\
\hline 2075.000 & 1. $28 \mathrm{E}-22$ & -------- & -------- & $6.59 \mathrm{E}-23$ & $8.74 E-26$ & -------- & $2.61 \mathrm{E}-23$ & ------- & \\
\hline 2175.000 & $2.11 \mathrm{E}-22$ & -------- & -------- & $9.62 \mathrm{E}-23$ & ------- & -------- & $3.81 \mathrm{E}-23$ & -------- & \\
\hline 2275.000 & $3.33 E-22$ & $1.58 \mathrm{E}-25$ & ------- & $1.46 \mathrm{E}-22$ & $1.05 \mathrm{E}-25$ & & $5.72 \mathrm{E}-23$ & & \\
\hline 2375.000 & $5.15 \mathrm{E}-22$ & $2.38 E-25$ & -------- & $2.08 E-22$ & ------- & -------- & $7.89 E-23$ & ------- & \\
\hline 2475.000 & $8.08 \mathrm{E}-22$ & -------- & -------- & $3.02 \mathrm{E}-22$ & 1. $92 \mathrm{E}-25$ & ------- & 1. $13 \mathrm{E}-22$ & $1.06 \mathrm{E}-25$ & \\
\hline 3925.000 & 1. $93 \mathrm{E}-22$ & $8.45 E-24$ & ------- & 1. $13 \mathrm{E}-22$ & $6.21 \mathrm{E}-24$ & & $5.23 E-23$ & $2.31 \mathrm{E}-24$ & \\
\hline 4125.000 & 1. $36 \mathrm{E}-22$ & $1.40 \mathrm{E}-23$ & ------ & $8.29 \mathrm{E}-23$ & $1.00 \mathrm{E}-23$ & -------- & $3.82 \mathrm{E}-23$ & $4.36 \mathrm{E}-24$ & \\
\hline 4325.000 & $9.86 \mathrm{E}-23$ & $2.59 \mathrm{E}-23$ & ------- & $6.19 E-23$ & $1.75 \mathrm{E}-23$ & & $2.89 \mathrm{E}-23$ & $E-24$ & \\
\hline 4525.000 & $7.17 \mathrm{E}-23$ & $4.79 E-23$ & -------- & $4.63 E-23$ & $3.06 \mathrm{E}-23$ & & $2.31 \mathrm{E}-23$ & 1. $25 \mathrm{E}-23$ & \\
\hline 4725.000 & $5.67 \mathrm{E}-23$ & 8. $95 \mathrm{E}-23$ & ------- & $3.72 \mathrm{E}-23$ & $5.22 \mathrm{E}-23$ & ------- & $1.76 \mathrm{E}-23$ & $2.04 \mathrm{E}-23$ & \\
\hline 4925.000 & $4.37 E-23$ & 1. $62 \mathrm{E}-22$ & ------- & $2.98 \mathrm{E}-23$ & $8.57 \mathrm{E}-23$ & -- & $1.49 \mathrm{E}-23$ & $E-23$ & \\
\hline 5125.000 & $3.57 \mathrm{E}-23$ & $2.87 \mathrm{E}-22$ & ------- & $2.38 \mathrm{E}-23$ & 1. $36 \mathrm{E}-22$ & & $1.17 \mathrm{E}-23$ & $5.05 E-23$ & \\
\hline 5325.000 & $2.91 \mathrm{E}-23$ & $4.71 \mathrm{E}-22$ & ------- & $2.00 \mathrm{E}-23$ & $2.05 E-22$ & ------ & $1.01 \mathrm{E}-23$ & $7.23 E-23$ & - \\
\hline 5525.000 & $2.29 \mathrm{E}-23$ & $5.41 \mathrm{E}-22$ & ------- & $1.73 \mathrm{E}-23$ & $2.44 \mathrm{E}-22$ & ----- & $8.30 E-24$ & $8.67 \mathrm{E}-23$ & \\
\hline 5725.000 & $2.02 \mathrm{E}-23$ & 4. $30 \mathrm{E}-22$ & ------- & 1. $40 \mathrm{E}-23$ & $2.09 \mathrm{E}-22$ & & $6.99 \mathrm{E}-24$ & $7.72 \mathrm{E}-23$ & \\
\hline 5925.000 & $1.81 \mathrm{E}-23$ & 3. $24 \mathrm{E}-22$ & ------- & 1. $23 \mathrm{E}-23$ & 1. $72 \mathrm{E}-22$ & ------- & $5.94 \mathrm{E}-24$ & $6.66 \mathrm{E}-23$ & - \\
\hline 6125.000 & $1.66 \mathrm{E}-23$ & $2.41 \mathrm{E}-22$ & ------- & $1.00 \mathrm{E}-23$ & 1. $37 \mathrm{E}-22$ & ----- & $5.39 \mathrm{E}-24$ & $5.43 E-23$ & \\
\hline 6325.000 & $1.41 \mathrm{E}-23$ & $1.71 \mathrm{E}-22$ & -------- & $9.36 \mathrm{E}-24$ & $1.05 \mathrm{E}-22$ & -------- & $4.71 \mathrm{E}-24$ & $4.34 \mathrm{E}-23$ & \\
\hline 6525.000 & 1. $24 \mathrm{E}-23$ & 1. $20 \mathrm{E}-22$ & -------- & $8.75 E-24$ & $7.91 \mathrm{E}-23$ & ------- & $4.02 E-24$ & $3.46 \mathrm{E}-23$ & ------- \\
\hline 6875.000 & $9.46 \mathrm{E}-24$ & $6.89 E-23$ & -------- & $6.63 \mathrm{E}-24$ & $5.05 E-23$ & -------- & $3.57 \mathrm{E}-24$ & $2.19 \mathrm{E}-23$ & -- \\
\hline 7375.000 & $7.88 \mathrm{E}-24$ & $3.36 \mathrm{E}-23$ & -------- & $5.02 E-24$ & $2.67 \mathrm{E}-23$ & -------- & $2.52 \mathrm{E}-24$ & $1.21 \mathrm{E}-23$ & \\
\hline 7875.000 & $6.26 \mathrm{E}-24$ & $1.86 \mathrm{E}-23$ & $2.67 \mathrm{E}-26$ & $4.02 \mathrm{E}-24$ & $1.49 \mathrm{E}-23$ & $3.86 \mathrm{E}-26$ & $2.07 \mathrm{E}-24$ & $7.25 \mathrm{E}-24$ & ------- \\
\hline 8375.000 & $5.07 \mathrm{E}-24$ & $1.09 \mathrm{E}-23$ & $3.56 \mathrm{E}-26$ & $3.29 \mathrm{E}-24$ & $9.51 \mathrm{E}-24$ & $1.78 \mathrm{E}-26$ & $1.73 \mathrm{E}-24$ & $4.42 \mathrm{E}-24$ & ------- \\
\hline 8875.000 & $4.14 \mathrm{E}-24$ & $7.30 \mathrm{E}-24$ & $8.45 E-26$ & $2.91 \mathrm{E}-24$ & $6.28 \mathrm{E}-24$ & $6.54 \mathrm{E}-26$ & 1. $34 \mathrm{E}-24$ & $2.99 \mathrm{E}-24$ & $3.62 \mathrm{E}-26$ \\
\hline 9375.000 & 3. $33 E-24$ & $4.92 \mathrm{E}-24$ & $1.51 \mathrm{E}-25$ & $2.46 \mathrm{E}-24$ & $4.39 \mathrm{E}-24$ & $1.40 \mathrm{E}-25$ & 1. $22 \mathrm{E}-24$ & $2.24 \mathrm{E}-24$ & $6.46 \mathrm{E}-26$ \\
\hline 9875.000 & $2.89 E-24$ & $3.45 \mathrm{E}-24$ & $3.38 \mathrm{E}-25$ & $2.06 \mathrm{E}-24$ & $3.46 \mathrm{E}-24$ & $3.06 \mathrm{E}-25$ & $1.09 \mathrm{E}-24$ & 1. $63 \mathrm{E}-24$ & 1. $22 \mathrm{E}-25$ \\
\hline
\end{tabular}


Table 31 (continued)

$\begin{array}{llll}16375.000 & 9.35 \mathrm{E}-25 & 5.07 \mathrm{E}-25 & 9.71 \mathrm{E}-24\end{array}$ $17125.000 \quad 7.72 \mathrm{E}-25 \quad 4.57 \mathrm{E}-25 \quad 4.94 \mathrm{E}-24$ $18125.000 \quad 5.40 \mathrm{E}-25 \quad 3.83 \mathrm{E}-25 \quad 2.28 \mathrm{E}-24$ $\begin{array}{llll}19125.000 & 5.89 \mathrm{E}-25 & 2.48 \mathrm{E}-25 & 1.39 \mathrm{E}-24\end{array}$ $20125.000 \quad 4.64 \mathrm{E}-25 \quad 2.06 \mathrm{E}-25 \quad 8.99 \mathrm{E}-25$ $21125.000 \quad 3.71 \mathrm{E}-25 \quad 2.30 \mathrm{E}-25 \quad 6.07 \mathrm{E}-25$ $22125.000 \quad 3.36 \mathrm{E}-25 \quad 2.14 \mathrm{E}-25 \quad 3.38 \mathrm{E}-25$ $23125.000 \quad 2.84 \mathrm{E}-25 \quad 1.43 \mathrm{E}-25 \quad 3.05 \mathrm{E}-25$ $24125.000 \quad 1.45 \mathrm{E}-25 \quad 1.53 \mathrm{E}-25 \quad 2.31 \mathrm{E}-25$ $25125.000 \quad 6.24 \mathrm{E}-26 \quad 1.45 \mathrm{E}-25 \quad 2.60 \mathrm{E}-25$ 26125.000 ------- $1.14 \mathrm{E}-25 \quad 1.76 \mathrm{E}-25$ 27125.000 ------- $1.14 \mathrm{E}-25 \quad 1.85 \mathrm{E}-25$ 28125.000 ------- $1.32 \mathrm{E}-25 \quad 1.31 \mathrm{E}-25$ 29125.000 ------- $1.24 \mathrm{E}-25 \quad 1.16 \mathrm{E}-25$ 30125.000 ------- $1.14 \mathrm{E}-25 \quad 1.07 \mathrm{E}-25$ 31125.000 ------ 8.19E-26 1.07E-25 32125.000 ------ 8.45E-26 $1.09 \mathrm{E}-25$ 33125.000 ------ 5.55E-26 5.56E-26 34125.000 ------ 7.40E-26 $6.45 \mathrm{E}-26$ 35125.000 ------- 6.60E-26 $4.45 \mathrm{E}-26$ 36125.000 ------ 7.40E-26 6.45E-26 37875.000 ------- 5.39E-26 $4.45 \mathrm{E}-26$ $40375.000 \quad------4.65 E-26 \quad 3.83 E-26$ 42875.000 ------- 3.59E-26 $3.47 \mathrm{E}-26$ 45375.000 ------- 4.01E-26 2.76E-26 47875.000 ------ $1.90 \mathrm{E}-26 \quad 2.05 \mathrm{E}-26$ 50375.000 ------ $8.45 \mathrm{E}-27 \quad 1.16 \mathrm{E}-26$ 52875.000 -------- -------- $1.87 \mathrm{E}-26$ 55375.000 -57875.000 ------- ------ 8.90E-27 60375.000 -------- -------- $1.16 \mathrm{E}-26$ 62875.000 -------- ------- 1.60E-26 65375.000 ------- ------- 7.12E-27 67875.000 -------- ------- 8.90E-27 70375.000 -------- ------- 7.12E-27 72875.000 ------- ------ 1.16E-26
$6.09 \mathrm{E}-25 \quad 4.69 \mathrm{E}-25 \quad 6.88 \mathrm{E}-24$ $4.36 \mathrm{E}-25 \quad 4.00 \mathrm{E}-25 \quad 3.56 \mathrm{E}-24$ 3.79E-25 2.87E-25 1.92E-24 $3.42 \mathrm{E}-25 \quad 2.43 \mathrm{E}-25 \quad 1.09 \mathrm{E}-24$ 3. $07 \mathrm{E}-25 \quad 2.50 \mathrm{E}-25 \quad 7.37 \mathrm{E}-25$ $2.47 \mathrm{E}-25 \quad 1.94 \mathrm{E}-25 \quad 4.47 \mathrm{E}-25$ $1.95 \mathrm{E}-25 \quad 1.85 \mathrm{E}-25 \quad 3.45 \mathrm{E}-25$ $\begin{array}{lll}1.40 \mathrm{E}-25 & 1.47 \mathrm{E}-25 & 2.24 \mathrm{E}-25\end{array}$ $8.98 \mathrm{E}-26 \quad 1.63 \mathrm{E}-25 \quad 1.92 \mathrm{E}-25$ $4.16 \mathrm{E}-26 \quad 1.22 \mathrm{E}-25 \quad 1.63 \mathrm{E}-25$ $2.63 \mathrm{E}-26 \quad 1.21 \mathrm{E}-25 \quad 1.14 \mathrm{E}-25$ 1.09E-26 9.79E-26 $9.81 \mathrm{E}-26$ ------ 7.69E-26 1.04E-25 ------- 8.92E-26 8.92E-26 ------- 8.22E-26 7.58E-26 ------- 7.52E-26 6.99E-26 ------ 5.95E-26 6.39E-26 ------- 6.65E-26 6.39E-26 ------ 6.47E-26 4.16E-26 ------- 8.22E-26 4.91E-26 ------- 4.20E-26 4.91E-26 ------ 4.27E-26 4.04E-26 ------- 3.99E-26 2.26E-26 ------- 2.73E-26 2.26E-26 ------- 3.29E-26 2.08E-26 ------- $1.96 \mathrm{E}-26 \quad 2.38 \mathrm{E}-26$ ------- 3.50E-27 1.25E-26

-------- -------- 1.61E-26 ------- ------- 9.51E-27 ------- ------- 9.51E-27 ------- ------- 8.32E-27 ------ ------ 7.13E-27 ------- ------- 8.32E-27 ------- ------- 1.07E-26 ------- ------ 5.95E-27 ------- ------ $2.97 \mathrm{E}-27$
$2.56 \mathrm{E}-25 \quad 2.57 \mathrm{E}-25 \quad 3.11 \mathrm{E}-24$ $2.65 \mathrm{E}-25 \quad 1.86 \mathrm{E}-25 \quad 1.75 \mathrm{E}-24$ 1.63E-25 $1.72 \mathrm{E}-25 \quad 8.63 \mathrm{E}-25$ $1.76 \mathrm{E}-25 \quad 1.34 \mathrm{E}-25 \quad 4.96 \mathrm{E}-25$ $\begin{array}{lll}1.39 \mathrm{E}-25 & 9.81 \mathrm{E}-26 & 3.22 \mathrm{E}-25\end{array}$ $\begin{array}{lll}1.46 \mathrm{E}-25 & 1.06 \mathrm{E}-25 & 2.47 \mathrm{E}-25\end{array}$ $\begin{array}{llll}1.24 \mathrm{E}-25 & 6.64 \mathrm{E}-26 & 2.22 \mathrm{E}-25\end{array}$ $4.44 \mathrm{E}-26 \quad 8.45 \mathrm{E}-26 \quad 1.27 \mathrm{E}-25$ $\begin{array}{llll}4.08 \mathrm{E}-26 & 6.04 \mathrm{E}-26 & 9.18 \mathrm{E}-26\end{array}$ $1.78 \mathrm{E}-26 \quad 6.49 \mathrm{E}-26 \quad 8.66 \mathrm{E}-26$ ------ 5.89E-26 5.43E-26 ------ 5.89E-26 5.04E-26 ------- 6.19E-26 5.43E-26 ------- $4.38 \mathrm{E}-26 \quad 3.75 \mathrm{E}-26$ ------- 3.92E-26 4.14E-26 ------ 3.92E-26 2.07E-26 ------ 3.92E-26 3.36E-26 ------- 3.32E-26 2.46E-26 ------- 2.42E-26 $1.94 \mathrm{E}-26$ ------ 2.11E-26 1.29E-26 ------- 2.57E-26 $1.16 \mathrm{E}-26$ ------ 2.17E-26 $1.71 \mathrm{E}-26$ ------ 1.15E-26 1.71E-26 ------- 1.09E-26 1.14E-26 ------- $1.09 \mathrm{E}-26 \quad 8.27 \mathrm{E}-27$ ------- 8.45E-27 7.24E-27 ------ ------ 6.20E-27 ------- ------- 5.17E-27 ------- ------- 5.17E-27 ------- ------ 5.69E-27 ------- ------- 4.14E-27 ------- ------ $4.65 \mathrm{E}-27$ -------- -------- -------------- ------ 3.10E-27 -------- -------- ------- 
Table 32

The singly differential cross section as a function of the ejected electron energy for single stripping in 5000-25000 keV/u 0^6+, $0^{\wedge} 7^{++}$ H_2 collisions.

\begin{tabular}{|c|c|c|c|c|c|c|}
\hline \multirow{2}{*}{$\begin{array}{l}\text { Electron } \\
\text { Energy }(\mathrm{eV})\end{array}$} & \multicolumn{6}{|c|}{ Ion Energy $(\mathrm{keV} / \mathrm{u})$} \\
\hline & 5000 & 10000 & 25000 & 5000 & 10000 & 25000 \\
\hline 350.000 & $1.01 \mathrm{E}-25$ & -------- & -------- & -------- & -------- & ------- \\
\hline 400.000 & 1. $24 \mathrm{E}-25$ & ------- & ------- & $5.28 \mathrm{E}-26$ & ------- & ------- \\
\hline 450.000 & $6.76 \mathrm{E}-26$ & ------- & ------- & $5.28 \mathrm{E}-26$ & ------- & ------- \\
\hline 500.000 & $2.59 \mathrm{E}-25$ & ------- & ------- & $5.28 \mathrm{E}-26$ & ------- & ------- \\
\hline 550.000 & $2.14 \mathrm{E}-25$ & ------- & ------- & $8.44 \mathrm{E}-26$ & ------- & ------- \\
\hline 600.000 & $1.58 \mathrm{E}-25$ & -------- & -------- & $7.39 \mathrm{E}-26$ & -------- & -------- \\
\hline 675.000 & $2.87 \mathrm{E}-25$ & -------- & -------- & $1.16 \mathrm{E}-25$ & -------- & -------- \\
\hline 775.000 & $3.44 \mathrm{E}-25$ & -------- & -------- & $1.53 \mathrm{E}-25$ & -------- & -------- \\
\hline 875.000 & $5.35 \mathrm{E}-25$ & $1.69 \mathrm{E}-26$ & ------ & $1.85 \mathrm{E}-25$ & ------- & ------- \\
\hline 975.000 & $6.76 \mathrm{E}-25$ & ------- & ------- & $2.11 \mathrm{E}-25$ & ------- & ------- \\
\hline 1075.000 & $7.72 \mathrm{E}-25$ & $2.37 \mathrm{E}-26$ & ------- & $2.53 \mathrm{E}-25$ & ------- & ------- \\
\hline 1175.000 & $1.09 \mathrm{E}-24$ & $2.03 \mathrm{E}-26$ & ------ & $4.22 \mathrm{E}-25$ & $2.22 \mathrm{E}-26$ & ------ \\
\hline 1275.000 & 1. $27 \mathrm{E}-24$ & $2.37 \mathrm{E}-26$ & ------ & $5.49 \mathrm{E}-25$ & $1.58 \mathrm{E}-26$ & ---- \\
\hline 1375.000 & $1.62 \mathrm{E}-24$ & $3.72 \mathrm{E}-26$ & ----- & $6.07 \mathrm{E}-25$ & ----- & --- \\
\hline 1475.000 & $2.09 \mathrm{E}-24$ & $5.07 \mathrm{E}-26$ & ------ & $7.86 \mathrm{E}-25$ & ------ & ---- \\
\hline 1575.000 & $2.36 \mathrm{E}-24$ & $5.41 \mathrm{E}-26$ & ------ & $8.87 \mathrm{E}-25$ & 2. $22 \mathrm{E}-26$ & ------- \\
\hline 1675.000 & $2.94 \mathrm{E}-24$ & $8.11 \mathrm{E}-26$ & ------- & $1.06 \mathrm{E}-24$ & $2.53 \mathrm{E}-26$ & ------- \\
\hline 1775.000 & $3.54 \mathrm{E}-24$ & $3.72 \mathrm{E}-26$ & ------ & $1.35 \mathrm{E}-24$ & $3.17 \mathrm{E}-26$ & ------- \\
\hline 1875.000 & $4.24 \mathrm{E}-24$ & $9.47 \mathrm{E}-26$ & ------ & $1.56 \mathrm{E}-24$ & $2.22 \mathrm{E}-26$ & ------ \\
\hline 1975.000 & $5.12 \mathrm{E}-24$ & $1.15 \mathrm{E}-25$ & ------ & $1.84 \mathrm{E}-24$ & $4.12 \mathrm{E}-26$ & ------- \\
\hline 2075.000 & $6.07 \mathrm{E}-24$ & $1.15 \mathrm{E}-25$ & ------- & $2.31 \mathrm{E}-24$ & $4.43 \mathrm{E}-26$ & ------- \\
\hline 2175.000 & $7.38 \mathrm{E}-24$ & $1.62 \mathrm{E}-25$ & ------ & $2.59 \mathrm{E}-24$ & $5.07 \mathrm{E}-26$ & ----- \\
\hline 2275.000 & $8.39 \mathrm{E}-24$ & $1.42 \mathrm{E}-25$ & ----- & $2.88 \mathrm{E}-24$ & 1. $23 \mathrm{E}-25$ & --- \\
\hline 2375.000 & $9.36 \mathrm{E}-24$ & $2.23 \mathrm{E}-25$ & ------ & $3.46 \mathrm{E}-24$ & $5.70 \mathrm{E}-26$ & ------ \\
\hline 2475.000 & $1.14 \mathrm{E}-23$ & $2.23 \mathrm{E}-25$ & ------ & $4.05 \mathrm{E}-24$ & $8.23 \mathrm{E}-26$ & ------- \\
\hline 2575.000 & 1. $37 \mathrm{E}-23$ & $2.70 \mathrm{E}-25$ & ----- & $4.99 \mathrm{E}-24$ & $9.50 \mathrm{E}-26$ & ---- \\
\hline 2725.000 & $1.98 \mathrm{E}-23$ & $3.31 \mathrm{E}-25$ & ------- & $6.70 \mathrm{E}-24$ & $1.22 \mathrm{E}-25$ & ---- \\
\hline 2925.000 & $2.44 \mathrm{E}-23$ & $3.92 \mathrm{E}-25$ & -------- & $8.90 \mathrm{E}-24$ & $1.44 \mathrm{E}-25$ & -------- \\
\hline 3125.000 & $2.56 \mathrm{E}-23$ & $5.95 \mathrm{E}-25$ & ------- & $9.05 \mathrm{E}-24$ & $2.44 \mathrm{E}-25$ & ------- \\
\hline 3325.000 & $2.58 \mathrm{E}-23$ & $7.81 \mathrm{E}-25$ & ------- & $9.76 \mathrm{E}-24$ & $3.40 \mathrm{E}-25$ & ------- \\
\hline 3525.000 & $2.50 \mathrm{E}-23$ & $1.02 \mathrm{E}-24$ & ------ & $9.88 \mathrm{E}-24$ & $4.05 \mathrm{E}-25$ & ------ \\
\hline 3725.000 & $2.41 \mathrm{E}-23$ & $1.36 \mathrm{E}-24$ & ------ & $9.54 \mathrm{E}-24$ & $5.08 \mathrm{E}-25$ & ------- \\
\hline 3925.000 & $2.27 \mathrm{E}-23$ & $1.70 \mathrm{E}-24$ & -- & $9.09 \mathrm{E}-24$ & $6.02 \mathrm{E}-25$ & --- \\
\hline 4125.000 & $2.11 \mathrm{E}-23$ & $2.14 \mathrm{E}-24$ & - & $8.27 \mathrm{E}-24$ & $8.85 \mathrm{E}-25$ & ---- \\
\hline 4325.000 & $1.99 \mathrm{E}-23$ & $2.66 \mathrm{E}-24$ & -- & $8.21 \mathrm{E}-24$ & $1.10 \mathrm{E}-24$ & --- \\
\hline 4525.000 & $1.82 \mathrm{E}-23$ & $3.30 \mathrm{E}-24$ & -- & $7.57 \mathrm{E}-24$ & 1. $34 \mathrm{E}-24$ & --.-- \\
\hline 4725.000 & $1.66 \mathrm{E}-23$ & $4.25 \mathrm{E}-24$ & --- & $6.92 \mathrm{E}-24$ & $1.64 \mathrm{E}-24$ & ---- \\
\hline 4925.000 & $1.53 \mathrm{E}-23$ & $5.13 \mathrm{E}-24$ & ------- & $6.68 \mathrm{E}-24$ & $1.88 \mathrm{E}-24$ & ------- \\
\hline 5125.000 & $1.43 \mathrm{E}-23$ & $6.56 \mathrm{E}-24$ & $6.34 \mathrm{E}-27$ & $6.00 \mathrm{E}-24$ & $2.22 \mathrm{E}-24$ & ------- \\
\hline 5325.000 & $1.31 \mathrm{E}-23$ & $8.03 \mathrm{E}-24$ & ------ & $5.49 \mathrm{E}-24$ & $2.88 \mathrm{E}-24$ & ------- \\
\hline 5525.000 & $1.20 \mathrm{E}-23$ & $9.89 \mathrm{E}-24$ & $7.40 \mathrm{E}-27$ & $4.98 \mathrm{E}-24$ & $3.55 \mathrm{E}-24$ & ------- \\
\hline 5725.000 & $1.05 \mathrm{E}-23$ & $1.09 \mathrm{E}-23$ & $7.40 \mathrm{E}-27$ & $4.86 \mathrm{E}-24$ & $3.95 \mathrm{E}-24$ & ---- \\
\hline 5925.000 & $9.84 \mathrm{E}-24$ & $1.10 \mathrm{E}-23$ & $5.28 \mathrm{E}-27$ & $4.41 \mathrm{E}-24$ & $4.16 \mathrm{E}-24$ & ------- \\
\hline 6125.000 & $9.15 \mathrm{E}-24$ & $1.09 \mathrm{E}-23$ & $1.06 \mathrm{E}-26$ & $3.99 \mathrm{E}-24$ & $4.15 \mathrm{E}-24$ & $8.45 \mathrm{E}-27$ \\
\hline 6325.000 & $8.21 \mathrm{E}-24$ & $1.07 \mathrm{E}-23$ & $1.80 \mathrm{E}-26$ & $3.76 \mathrm{E}-24$ & $3.99 \mathrm{E}-24$ & --.-- \\
\hline 6525.000 & $7.61 \mathrm{E}-24$ & $1.02 \mathrm{E}-23$ & $1.59 \mathrm{E}-26$ & $3.48 \mathrm{E}-24$ & $3.86 \mathrm{E}-24$ & ------- \\
\hline 6875.000 & $6.54 \mathrm{E}-24$ & $9.48 \mathrm{E}-24$ & $2.32 \mathrm{E}-26$ & $3.08 \mathrm{E}-24$ & $3.77 \mathrm{E}-24$ & $9.72 \mathrm{E}-27$ \\
\hline 7375.000 & $5.42 \mathrm{E}-24$ & $8.17 \mathrm{E}-24$ & $2.75 \mathrm{E}-26$ & $2.58 \mathrm{E}-24$ & $3.27 \mathrm{E}-24$ & $1.52 \mathrm{E}-26$ \\
\hline 7875.000 & $4.51 \mathrm{E}-24$ & $6.86 \mathrm{E}-24$ & $4.18 \mathrm{E}-26$ & $2.12 \mathrm{E}-24$ & $2.80 \mathrm{E}-24$ & $2.03 \mathrm{E}-26$ \\
\hline 8375.000 & $3.80 \mathrm{E}-24$ & $5.58 \mathrm{E}-24$ & $7.73 \mathrm{E}-26$ & $1.81 \mathrm{E}-24$ & $2.41 \mathrm{E}-24$ & $3.55 \mathrm{E}-26$ \\
\hline 8875.000 & $3.18 \mathrm{E}-24$ & $4.63 \mathrm{E}-24$ & $1.15 \mathrm{E}-25$ & $1.55 \mathrm{E}-24$ & $1.98 \mathrm{E}-24$ & $5.33 \mathrm{E}-26$ \\
\hline 9375.000 & $2.76 \mathrm{E}-24$ & $3.80 \mathrm{E}-24$ & $1.64 \mathrm{E}-25$ & 1. $32 \mathrm{E}-24$ & $1.72 \mathrm{E}-24$ & $7.44 \mathrm{E}-26$ \\
\hline 9875.000 & $2.35 \mathrm{E}-24$ & $3.12 \mathrm{E}-24$ & $2.51 \mathrm{E}-25$ & $1.16 \mathrm{E}-24$ & $1.46 \mathrm{E}-24$ & $1.09 \mathrm{E}-25$ \\
\hline 10375.000 & 1. $92 \mathrm{E}-24$ & $2.66 \mathrm{E}-24$ & $3.83 \mathrm{E}-25$ & $9.21 \mathrm{E}-25$ & 1. $22 \mathrm{E}-24$ & $1.70 \mathrm{E}-25$ \\
\hline 10875.000 & $1.58 \mathrm{E}-24$ & $2.21 \mathrm{E}-24$ & $5.29 \mathrm{E}-25$ & $7.67 \mathrm{E}-25$ & $1.01 \mathrm{E}-24$ & $2.30 \mathrm{E}-25$ \\
\hline 11375.000 & 1. $23 \mathrm{E}-24$ & $1.90 \mathrm{E}-24$ & $7.79 \mathrm{E}-25$ & $6.48 \mathrm{E}-25$ & $9.02 \mathrm{E}-25$ & $3.31 \mathrm{E}-25$ \\
\hline 11875.000 & $1.11 \mathrm{E}-24$ & $1.59 \mathrm{E}-24$ & $1.05 \mathrm{E}-24$ & $5.60 \mathrm{E}-25$ & $7.60 \mathrm{E}-25$ & $4.49 \mathrm{E}-25$ \\
\hline 12375.000 & $9.73 \mathrm{E}-25$ & $1.39 \mathrm{E}-24$ & $1.47 \mathrm{E}-24$ & $4.41 \mathrm{E}-25$ & $6.42 \mathrm{E}-25$ & $5.95 \mathrm{E}-25$ \\
\hline 12875.000 & $8.36 \mathrm{E}-25$ & $1.22 \mathrm{E}-24$ & $1.93 \mathrm{E}-24$ & $4.01 \mathrm{E}-25$ & $5.59 \mathrm{E}-25$ & $7.41 \mathrm{E}-25$ \\
\hline 13375.000 & $7.64 \mathrm{E}-25$ & $1.01 \mathrm{E}-24$ & $2.54 \mathrm{E}-24$ & $4.21 \mathrm{E}-25$ & $4.95 \mathrm{E}-25$ & $1.01 \mathrm{E}-24$ \\
\hline 13875.000 & $7.45 \mathrm{E}-25$ & $8.76 \mathrm{E}-25$ & $3.00 \mathrm{E}-24$ & $3.36 \mathrm{E}-25$ & $4.41 \mathrm{E}-25$ & $1.22 \mathrm{E}-24$ \\
\hline 14375.000 & $6.34 \mathrm{E}-25$ & $8.04 \mathrm{E}-25$ & $2.99 \mathrm{E}-24$ & $2.79 \mathrm{E}-25$ & $4.02 \mathrm{E}-25$ & 1. $20 \mathrm{E}-24$ \\
\hline 14875.000 & $5.78 \mathrm{E}-25$ & $7.32 \mathrm{E}-25$ & $2.84 \mathrm{E}-24$ & $2.51 \mathrm{E}-25$ & $3.32 \mathrm{E}-25$ & $1.20 \mathrm{E}-24$ \\
\hline 15375.000 & $4.78 \mathrm{E}-25$ & $6.39 \mathrm{E}-25$ & $2.62 \mathrm{E}-24$ & $2.54 \mathrm{E}-25$ & $3.24 \mathrm{E}-25$ & $1.09 \mathrm{E}-24$ \\
\hline 15875.000 & $4.49 \mathrm{E}-25$ & $5.55 \mathrm{E}-25$ & $2.28 \mathrm{E}-24$ & $2.02 \mathrm{E}-25$ & $2.88 \mathrm{E}-25$ & $1.00 \mathrm{E}-24$ \\
\hline
\end{tabular}


Table 32 (continued)

$16375.000 \quad 4.15 \mathrm{E}-25 \quad 5.50 \mathrm{E}-25 \quad 2.05 \mathrm{E}-24$ $17125.000 \quad 3.26 \mathrm{E}-25 \quad 4.44 \mathrm{E}-25 \quad 1.67 \mathrm{E}-24$ $18125.000 \quad 2.85 \mathrm{E}-25 \quad 3.72 \mathrm{E}-25 \quad 1.24 \mathrm{E}-24$ $19125.000 \quad 2.27 \mathrm{E}-25 \quad 3.06 \mathrm{E}-25 \quad 9.34 \mathrm{E}-25$ $20125.000 \quad 1.76 \mathrm{E}-25 \quad 2.75 \mathrm{E}-25 \quad 6.68 \mathrm{E}-25$ $21125.000 \quad 1.23 \mathrm{E}-25 \quad 2.42 \mathrm{E}-25 \quad 5.28 \mathrm{E}-25$ $22125.000 \quad 1.07 \mathrm{E}-25 \quad 1.82 \mathrm{E}-25 \quad 3.99 \mathrm{E}-25$ $23125.000 \quad 6.65 \mathrm{E}-26 \quad 1.67 \mathrm{E}-25 \quad 3.02 \mathrm{E}-25$ $24125.000 \quad 5.02 \mathrm{E}-26 \quad 1.55 \mathrm{E}-25 \quad 2.61 \mathrm{E}-25$ $25125.000 \quad 3.32 \mathrm{E}-26 \quad 1.36 \mathrm{E}-25 \quad 2.04 \mathrm{E}-25$ $26125.000 \quad 2.70 \mathrm{E}-26 \quad 1.17 \mathrm{E}-25 \quad 1.59 \mathrm{E}-25$ $27125.000 \quad 9.58 \mathrm{E}-27 \quad 1.00 \mathrm{E}-25 \quad 1.49 \mathrm{E}-25$ $28125.000 \quad 9.58 \mathrm{E}-27 \quad 9.23 \mathrm{E}-26 \quad 1.16 \mathrm{E}-25$ $29125.000 \quad 5.07 \mathrm{E}-27 \quad 8.79 \mathrm{E}-26 \quad 9.68 \mathrm{E}-26$ $30125.000 \quad----8.38 \mathrm{E}-26 \quad 8.43 \mathrm{E}-26$ 31125.000 ----- 7.78E-26 $7.71 \mathrm{E}-26$ 32125.000 ------ 5.88E-26 6.72E-26 33125.000 ------ 5.98E-26 5.18E-26 $34125.000-----5.17 \mathrm{E}-26 \quad 5.18 \mathrm{E}-26$ 35125.000 ------ $4.19 \mathrm{E}-26 \quad 4.80 \mathrm{E}-26$ 36125.000 ------- $4.16 \mathrm{E}-26 \quad 4.14 \mathrm{E}-26$ 37875.000 ------ 4.04E-26 3.49E-26 40375.000 ----- $3.21 \mathrm{E}-26 \quad 2.90 \mathrm{E}-26$ 42875.000 ------- 2.22E-26 2.39E-26 45375.000 ------ $1.20 \mathrm{E}-26 \quad 1.92 \mathrm{E}-26$ 47875.000 ------ 9.33E-27 $1.57 \mathrm{E}-26$ 50375.000 ----- $4.06 \mathrm{E}-27 \quad 1.36 \mathrm{E}-26$ $52875.000-------1.22 \mathrm{E}-27$ 1.23E-26 55375.000 ------- ------ 1.04E-26 57875.000 ------ ----- 8.20E-27 60375.000 ------- ------- 9.38E-27 62875.000 ------ ------ 8.88E-27 65375.000 ------- ------ 7.10E-27 67875.000 ------------ 5.07E-27 70375.000 ------- ------ 4.90E-27 72875.000 ------------- 5.24E-27
1.84E-25 2.51E-25 8.80E-25

$\begin{array}{ll}1.60 \mathrm{E}-25 & 2.33 \mathrm{E}-25 \quad 7.65 \mathrm{E}-25\end{array}$

1.36E-25 $2.06 \mathrm{E}-25 \quad 5.88 \mathrm{E}-25$

1.00E-25 $1.60 \mathrm{E}-25 \quad 4.40 \mathrm{E}-25$

$7.07 \mathrm{E}-26 \quad 1.31 \mathrm{E}-25 \quad 3.26 \mathrm{E}-25$

$5.70 \mathrm{E}-26 \quad 1.05 \mathrm{E}-25 \quad 2.57 \mathrm{E}-25$

4.96E-26 8.93E-26 2.05E-25

$3.17 \mathrm{E}-26 \quad 7.88 \mathrm{E}-26 \quad 1.53 \mathrm{E}-25$

$2.11 \mathrm{E}-26 \quad 7.25 \mathrm{E}-26 \quad 1.13 \mathrm{E}-25$

$1.48 \mathrm{E}-26 \quad 5.38 \mathrm{E}-26 \quad 1.04 \mathrm{E}-25$

$1.11 \mathrm{E}-26 \quad 5.19 \mathrm{E}-26 \quad 8.52 \mathrm{E}-26$

4.22E-27 5.07E-26 7.44E-26

$3.17 \mathrm{E}-27 \quad 4.69 \mathrm{E}-26 \quad 6.64 \mathrm{E}-26$

$3.17 \mathrm{E}-27 \quad 4.15 \mathrm{E}-26 \quad 5.22 \mathrm{E}-26$

------ 3.89E-26 3.89E-26

----- 4.05E-26 4.42E-26

----- 3.17E-26 3.02E-26

------ 2.44E-26 2.94E-26

------ 2.79E-26 2.92E-26

------ 2.41E-26 2.20E-26

------- 2.25E-26 2.30E-26

------ $1.94 \mathrm{E}-26 \quad 1.89 \mathrm{E}-26$

------ $1.38 \mathrm{E}-26 \quad 1.45 \mathrm{E}-26$

------ 1.15E-26 1.13E-26

----- 6.84E-27 $1.05 \mathrm{E}-26$

------ 4.05E-27 8.71E-27

----- 2.03E-27 7.95E-27

------- ------- 6.00E-27

----------- 6.09E-27

------ ----- 6.26E-27

------ ------ 4.06E-27

------- ------- 3.89E-27

------- ------- 2.87E-27

------- ------ $2.03 \mathrm{E}-27$

------ ------ 3.64E-27

-- $--------38 \mathrm{E}-27$ 
Table 33

The singly differential cross section as a function of the ejected electron angle for single stripping in 5000-25000 keV/u 0, $0^{\wedge}+, 0^{\wedge} 2++$ H_2 collisions.



Table 34

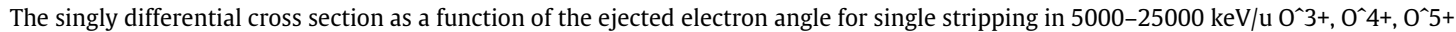
$+\mathrm{H} \_2$ collisions.

\begin{tabular}{|c|c|c|c|c|c|c|c|c|c|}
\hline \multirow{3}{*}{$\begin{array}{l}\text { Electron } \\
\text { Angle (deg) }\end{array}$} & \multicolumn{9}{|c|}{ Ion Energy $(\mathrm{keV} / \mathrm{u})$} \\
\hline & 5000 & 10000 & 25000 & 5000 & 10000 & 25000 & 5000 & 10000 & 25000 \\
\hline & & & & & & & & & \\
\hline 0.050 & $5.18 \mathrm{E}-17$ & $4.17 \mathrm{E}-17$ & $4.72 \mathrm{E}-17$ & $1.56 \mathrm{E}-17$ & $1.94 \mathrm{E}-17$ & $1.97 \mathrm{E}-17$ & $6.12 \mathrm{E}-18$ & $6.78 \mathrm{E}-18$ & $8.51 \mathrm{E}-18$ \\
\hline 0.300 & $6.81 \mathrm{E}-17$ & $5.40 \mathrm{E}-17$ & $5.65 \mathrm{E}-17$ & $2.23 \mathrm{E}-17$ & $2.25 \mathrm{E}-17$ & $2.04 \mathrm{E}-17$ & $7.67 \mathrm{E}-18$ & $7.41 \mathrm{E}-18$ & $6.71 \mathrm{E}-18$ \\
\hline 0.750 & $5.90 \mathrm{E}-17$ & $4.26 \mathrm{E}-17$ & 3. $92 \mathrm{E}-17$ & $1.97 \mathrm{E}-17$ & $1.77 \mathrm{E}-17$ & $1.42 \mathrm{E}-17$ & $7.06 \mathrm{E}-18$ & $5.95 \mathrm{E}-18$ & $4.89 \mathrm{E}-18$ \\
\hline 1.500 & $4.30 \mathrm{E}-17$ & $2.86 \mathrm{E}-17$ & $2.30 \mathrm{E}-17$ & $1.46 \mathrm{E}-17$ & 1. $21 \mathrm{E}-17$ & $8.70 \mathrm{E}-18$ & $5.12 \mathrm{E}-18$ & $4.17 \mathrm{E}-18$ & $3.03 E-18$ \\
\hline 2.500 & $3.14 \mathrm{E}-17$ & $1.85 \mathrm{E}-17$ & $1.18 \mathrm{E}-17$ & $1.09 \mathrm{E}-17$ & $8.26 \mathrm{E}-18$ & $5.03 E-18$ & $3.80 \mathrm{E}-18$ & $2.89 \mathrm{E}-18$ & $1.82 \mathrm{E}-18$ \\
\hline 3.500 & $2.31 \mathrm{E}-17$ & 1. $24 \mathrm{E}-17$ & $6.14 \mathrm{E}-18$ & 8. $31 \mathrm{E}-18$ & $5.88 \mathrm{E}-18$ & $2.95 \mathrm{E}-18$ & $3.00 \mathrm{E}-18$ & $2.09 E-18$ & 1. $11 \mathrm{E}-18$ \\
\hline 4.500 & $1.71 \mathrm{E}-17$ & $8.05 \mathrm{E}-18$ & $3.34 \mathrm{E}-18$ & $6.49 \mathrm{E}-18$ & $4.15 \mathrm{E}-18$ & $1.70 \mathrm{E}-18$ & $2.37 \mathrm{E}-18$ & $1.56 \mathrm{E}-18$ & $6.87 \mathrm{E}-19$ \\
\hline 5.500 & 1. $29 \mathrm{E}-17$ & $5.37 \mathrm{E}-18$ & $1.83 \mathrm{E}-18$ & $5.08 \mathrm{E}-18$ & $2.92 \mathrm{E}-18$ & $1.07 \mathrm{E}-18$ & $1.88 \mathrm{E}-18$ & 1. $11 \mathrm{E}-18$ & $4.48 \mathrm{E}-19$ \\
\hline 6.500 & $9.61 \mathrm{E}-18$ & $3.56 \mathrm{E}-18$ & 1. $12 \mathrm{E}-18$ & $3.96 \mathrm{E}-18$ & $2.09 \mathrm{E}-18$ & $6.64 \mathrm{E}-19$ & $1.49 \mathrm{E}-18$ & 8. $25 \mathrm{E}-19$ & $2.92 \mathrm{E}-19$ \\
\hline 7.500 & $7.20 \mathrm{E}-18$ & $2.51 \mathrm{E}-18$ & $6.82 \mathrm{E}-19$ & $3.12 \mathrm{E}-18$ & $1.53 \mathrm{E}-18$ & $4.43 E-19$ & 1. $20 \mathrm{E}-18$ & $6.10 \mathrm{E}-19$ & $1.87 \mathrm{E}-19$ \\
\hline 8.500 & $5.35 \mathrm{E}-18$ & 1. $73 \mathrm{E}-18$ & $4.63 \mathrm{E}-19$ & $2.47 \mathrm{E}-18$ & 1. $11 \mathrm{E}-18$ & $3.00 \mathrm{E}-19$ & $9.72 \mathrm{E}-19$ & $4.65 \mathrm{E}-19$ & 1. $29 \mathrm{E}-19$ \\
\hline 11.500 & $2.55 \mathrm{E}-18$ & $7.16 \mathrm{E}-19$ & $1.68 \mathrm{E}-19$ & 1. $27 \mathrm{E}-18$ & $4.96 \mathrm{E}-19$ & 1. $14 \mathrm{E}-19$ & 5. $20 \mathrm{E}-19$ & $2.16 \mathrm{E}-19$ & $5.38 \mathrm{E}-20$ \\
\hline 16.500 & $8.63 E-19$ & $2.09 E-19$ & $4.30 \mathrm{E}-20$ & $4.76 \mathrm{E}-19$ & 1. $54 \mathrm{E}-19$ & $3.05 \mathrm{E}-20$ & $2.15 \mathrm{E}-19$ & $7.26 \mathrm{E}-20$ & $1.44 \mathrm{E}-20$ \\
\hline 21.500 & $3.75 \mathrm{E}-19$ & 8. $23 \mathrm{E}-20$ & $1.63 \mathrm{E}-20$ & $2.21 \mathrm{E}-19$ & $6.64 \mathrm{E}-20$ & 1. $27 \mathrm{E}-20$ & $1.04 \mathrm{E}-19$ & 3. $20 \mathrm{E}-20$ & $5.95 E-21$ \\
\hline 26.500 & $2.04 \mathrm{E}-19$ & $4.45 \mathrm{E}-20$ & $8.94 \mathrm{E}-21$ & 1. $22 \mathrm{E}-19$ & $3.68 \mathrm{E}-20$ & $6.51 \mathrm{E}-21$ & $5.88 \mathrm{E}-20$ & $1.81 \mathrm{E}-20$ & $3.01 \mathrm{E}-21$ \\
\hline 31.500 & $6.93 \mathrm{E}-20$ & $1.42 \mathrm{E}-20$ & $2.50 \mathrm{E}-21$ & $4.36 \mathrm{E}-20$ & $1.16 \mathrm{E}-20$ & $1.99 \mathrm{E}-21$ & $2.15 \mathrm{E}-20$ & $6.15 \mathrm{E}-21$ & $1.01 \mathrm{E}-21$ \\
\hline 36.500 & $7.92 \mathrm{E}-21$ & $8.02 \mathrm{E}-22$ & $4.09 \mathrm{E}-23$ & $6.47 \mathrm{E}-21$ & $8.95 E-22$ & $6.38 \mathrm{E}-23$ & 3. $22 \mathrm{E}-21$ & $4.91 \mathrm{E}-22$ & ------- \\
\hline 41.500 & 1. $28 \mathrm{E}-21$ & $7.27 \mathrm{E}-23$ & ------- & $1.06 \mathrm{E}-21$ & $7.22 \mathrm{E}-23$ & -------- & $5.47 \mathrm{E}-22$ & 3. $32 E-23$ & ------- \\
\hline 46.500 & $3.05 E-22$ & -------- & ------- & $2.81 \mathrm{E}-22$ & $2.64 \mathrm{E}-23$ & -------- & 1. $16 \mathrm{E}-22$ & -------- & ------- \\
\hline 51.500 & 1. $37 \mathrm{E}-22$ & -------- & ------- & $5.61 \mathrm{E}-23$ & -------- & -------- & $5.79 E-23$ & -------- & -------- \\
\hline 56.500 & $5.30 \mathrm{E}-23$ & -------- & ------- & $2.87 E-23$ & -------- & -------- & $2.72 E-23$ & -------- & ------- \\
\hline 61.500 & ------- & ------- & ------- & $2.27 \mathrm{E}-23$ & -------- & -------- & -------- & -------- & ------- \\
\hline 66.500 & ------ & ------- & ------- & -------- & -------- & -------- & -------- & ------- & ------ \\
\hline 71.500 & ------ & -------- & ------- & -------- & -------- & -------- & -------- & -------- & ------- \\
\hline 76.500 & ------ & ------- & ------ & ------- & ------- & ------- & ------- & ------- & ------ \\
\hline 81.500 & ------- & -------- & -------- & -------- & -------- & -------- & -------- & -------- & 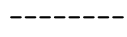 \\
\hline 86.500 & ------- & 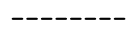 & ------- & -------- & -------- & -------- & -------- & -------- & - \\
\hline
\end{tabular}


Table 35

The singly differential cross section as a function of the ejected electron angle for single stripping in $5000-25000 \mathrm{keV} / \mathrm{u} \mathrm{O}^{\wedge} 6+, 0^{\wedge} 7++$ H_2 collisions.

\begin{tabular}{|c|c|c|c|c|c|c|}
\hline \multirow{2}{*}{$\begin{array}{l}\text { Electron } \\
\text { Angle (deg) }\end{array}$} & \multicolumn{6}{|c|}{ Ion Energy $(\mathrm{keV} / \mathrm{u})$} \\
\hline & 5000 & 10000 & 25000 & 5000 & 10000 & 25000 \\
\hline 0.050 & $6.48 \mathrm{E}-19$ & $6.36 \mathrm{E}-19$ & $7.29 \mathrm{E}-19$ & $4.96 \mathrm{E}-19$ & $4.30 \mathrm{E}-19$ & $2.87 \mathrm{E}-19$ \\
\hline 0.300 & 1. $10 \mathrm{E}-18$ & $8.30 \mathrm{E}-19$ & $6.50 \mathrm{E}-19$ & $4.76 \mathrm{E}-19$ & $3.05 \mathrm{E}-19$ & $2.64 \mathrm{E}-19$ \\
\hline 0.750 & $1.04 \mathrm{E}-18$ & $6.99 \mathrm{E}-19$ & $5.10 \mathrm{E}-19$ & $3.99 \mathrm{E}-19$ & $2.66 \mathrm{E}-19$ & $1.98 \mathrm{E}-19$ \\
\hline 1.500 & $8.74 \mathrm{E}-19$ & $5.97 \mathrm{E}-19$ & $3.88 \mathrm{E}-19$ & 3. $34 \mathrm{E}-19$ & $2.31 \mathrm{E}-19$ & $1.49 \mathrm{E}-19$ \\
\hline 2.500 & $7.10 \mathrm{E}-19$ & $4.77 \mathrm{E}-19$ & $2.84 \mathrm{E}-19$ & $2.93 \mathrm{E}-19$ & $1.81 \mathrm{E}-19$ & 1. $13 \mathrm{E}-19$ \\
\hline 3.500 & $6.06 \mathrm{E}-19$ & $3.82 \mathrm{E}-19$ & $2.20 \mathrm{E}-19$ & $2.46 \mathrm{E}-19$ & $1.48 \mathrm{E}-19$ & $8.67 E-20$ \\
\hline 4.500 & $5.33 E-19$ & $3.21 \mathrm{E}-19$ & $1.70 \mathrm{E}-19$ & $2.19 \mathrm{E}-19$ & 1. $28 \mathrm{E}-19$ & $7.09 \mathrm{E}-20$ \\
\hline 5.500 & $4.55 \mathrm{E}-19$ & $2.72 \mathrm{E}-19$ & 1. $33 \mathrm{E}-19$ & $1.83 \mathrm{E}-19$ & $1.04 \mathrm{E}-19$ & $5.40 \mathrm{E}-20$ \\
\hline 6.500 & $3.84 \mathrm{E}-19$ & $2.23 E-19$ & $1.05 \mathrm{E}-19$ & 1. $62 \mathrm{E}-19$ & $8.98 \mathrm{E}-20$ & $4.59 \mathrm{E}-20$ \\
\hline 7.500 & 3. $36 \mathrm{E}-19$ & 1. $89 \mathrm{E}-19$ & $8.30 \mathrm{E}-20$ & 1. $39 \mathrm{E}-19$ & $7.53 \mathrm{E}-20$ & $3.60 \mathrm{E}-20$ \\
\hline 8.500 & $2.85 E-19$ & $1.64 \mathrm{E}-19$ & $6.80 \mathrm{E}-20$ & 1. $18 \mathrm{E}-19$ & $6.34 \mathrm{E}-20$ & $2.87 \mathrm{E}-20$ \\
\hline 11.500 & $1.89 \mathrm{E}-19$ & $1.01 \mathrm{E}-19$ & $3.68 \mathrm{E}-20$ & $7.76 \mathrm{E}-20$ & $4.24 \mathrm{E}-20$ & $1.68 \mathrm{E}-20$ \\
\hline 16.500 & $1.01 \mathrm{E}-19$ & $5.12 \mathrm{E}-20$ & $1.52 \mathrm{E}-20$ & $4.15 \mathrm{E}-20$ & $2.16 \mathrm{E}-20$ & $7.31 E-21$ \\
\hline 21.500 & $5.83 E-20$ & $2.90 \mathrm{E}-20$ & $7.42 \mathrm{E}-21$ & $2.49 \mathrm{E}-20$ & 1. $28 \mathrm{E}-20$ & $3.77 \mathrm{E}-21$ \\
\hline 26.500 & $3.27 \mathrm{E}-20$ & $1.64 \mathrm{E}-20$ & $4.21 \mathrm{E}-21$ & 1. $39 \mathrm{E}-20$ & $7.11 \mathrm{E}-21$ & $2.09 \mathrm{E}-21$ \\
\hline 31.500 & $1.55 \mathrm{E}-20$ & $6.98 \mathrm{E}-21$ & $1.54 \mathrm{E}-21$ & $6.61 \mathrm{E}-21$ & $3.13 \mathrm{E}-21$ & $7.73 E-22$ \\
\hline 36.500 & $6.12 \mathrm{E}-21$ & $2.07 \mathrm{E}-21$ & $2.49 \mathrm{E}-22$ & $2.65 E-21$ & $9.57 \mathrm{E}-22$ & $1.49 \mathrm{E}-22$ \\
\hline 41.500 & $2.13 \mathrm{E}-21$ & $5.49 \mathrm{E}-22$ & $3.49 \mathrm{E}-23$ & $9.82 \mathrm{E}-22$ & $2.56 \mathrm{E}-22$ & $2.44 \mathrm{E}-23$ \\
\hline 46.500 & $8.68 \mathrm{E}-22$ & $1.74 \mathrm{E}-22$ & $7.97 \mathrm{E}-24$ & $3.48 \mathrm{E}-22$ & $8.12 \mathrm{E}-23$ & $2.66 \mathrm{E}-24$ \\
\hline 51.500 & 3. $32 \mathrm{E}-22$ & $5.12 \mathrm{E}-23$ & -------- & $1.87 \mathrm{E}-22$ & $3.03 E-23$ & -------- \\
\hline 56.500 & $1.91 \mathrm{E}-22$ & $2.00 \mathrm{E}-23$ & ------- & $7.04 \mathrm{E}-23$ & $1.04 \mathrm{E}-23$ & -------- \\
\hline 61.500 & $9.94 \mathrm{E}-23$ & $7.72 \mathrm{E}-24$ & -------- & $3.29 \mathrm{E}-23$ & $4.60 \mathrm{E}-24$ & -------- \\
\hline 66.500 & $5.72 \mathrm{E}-23$ & $6.72 \mathrm{E}-24$ & -------- & $9.45 \mathrm{E}-24$ & $4.41 \mathrm{E}-24$ & ------- \\
\hline 71.500 & $1.84 \mathrm{E}-23$ & $3.90 \mathrm{E}-24$ & ------- & $1.12 \mathrm{E}-23$ & ------ & ------ \\
\hline 76.500 & $2.33 E-23$ & ------ & ------- & $4.95 \mathrm{E}-24$ & ------- & ------- \\
\hline 81.500 & $1.45 E-23$ & ------ & ------- & ------- & ------ & ------ \\
\hline 86.500 & $6.18 E-24$ & ------ & ------ & $4.82 \mathrm{E}-24$ & ------ & ------ \\
\hline
\end{tabular}


Table 36

The singly differential cross section as a function of the ejected electron energy for double stripping in $5000-25000 \mathrm{keV} / \mathrm{u} \mathrm{O}, 0^{\wedge}+, 0^{\wedge} 2+$ $+\mathrm{H} \_2$ collisions.

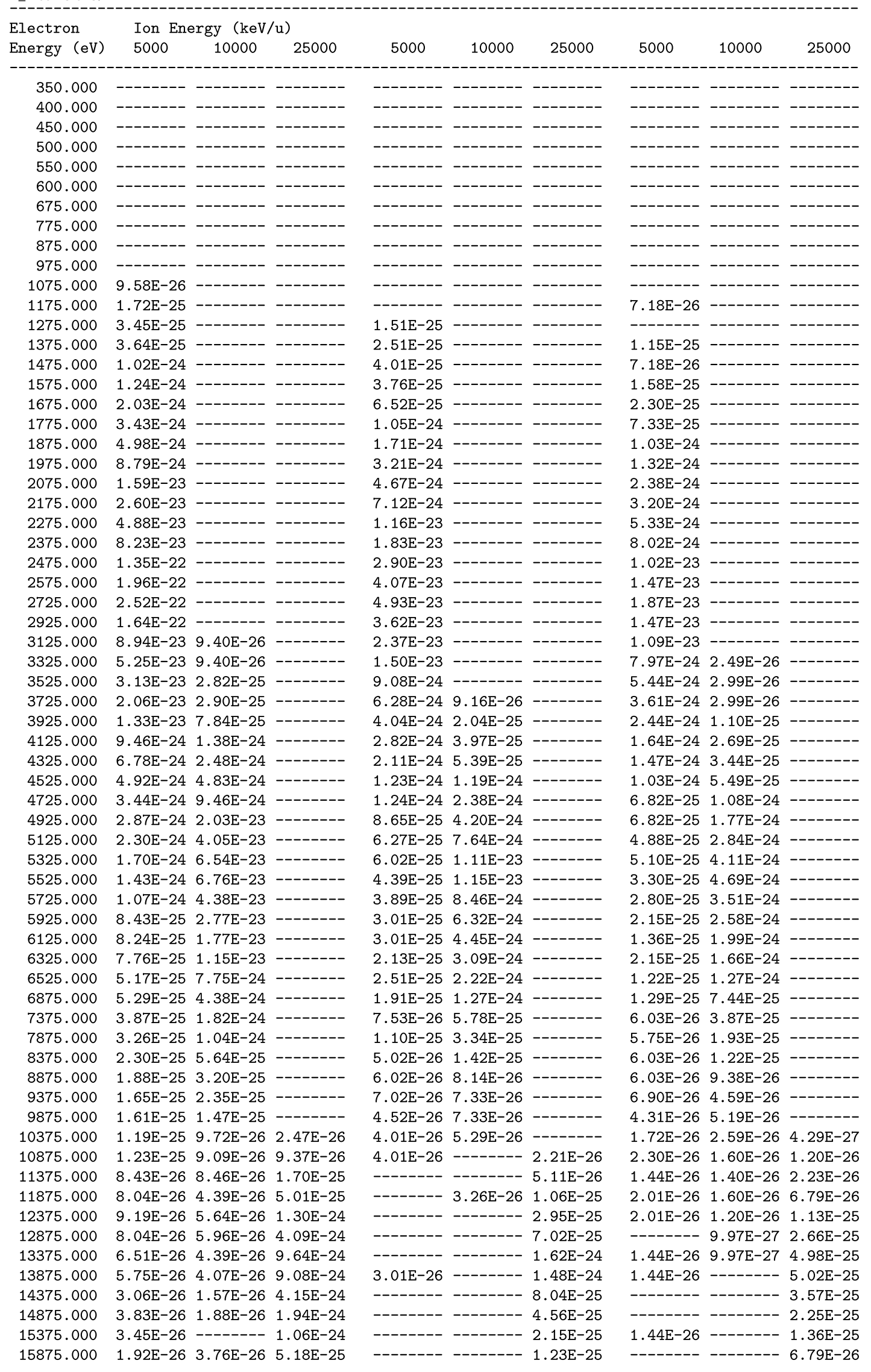


Table 36 (continued)

$16375.000 \quad 2.30 \mathrm{E}-26------2.66 \mathrm{E}-25$

$17125.000 \quad 3.06 \mathrm{E}-26 \quad 1.10 \mathrm{E}-26 \quad 1.46 \mathrm{E}-25$

$18125.000 \quad 2.30 \mathrm{E}-26 \quad 2.35 \mathrm{E}-26 \quad 8.01 \mathrm{E}-26$

$19125.000 \quad 2.68 \mathrm{E}-26 \quad 9.40 \mathrm{E}-27 \quad 3.82 \mathrm{E}-26$

$20125.000 \quad 2.68 \mathrm{E}-26 \quad 7.84 \mathrm{E}-27 \quad 2.22 \mathrm{E}-26$

$21125.000 \quad 1.53 \mathrm{E}-26$------- $1.48 \mathrm{E}-26$

$22125.000 \quad 9.58 \mathrm{E}-27-------8.63 \mathrm{E}-27$

23125.000 ------ ----- 6.17E-27

24125.000 ------ ----- 6.17E-27

25125.000

26125.000

27125.000

28125.000

29125.000

30125.000

31125.000

32125.000

33125.000

34125.000

35125.000

36125.000

37875.000

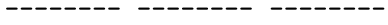

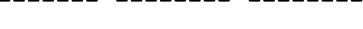

$-----------9.37 \mathrm{E}-26$

$2.01 \mathrm{E}-26-----4.51 \mathrm{E}-26$

------- ------- $1.62 \mathrm{E}-26$

------ ------ $1.28 \mathrm{E}-26$

------- -------- -------

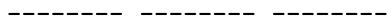

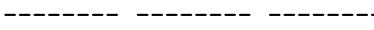

------- ------- -------

------- -------- -------

-------- -------- -------

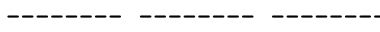

------- -------- -------

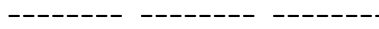

------- -------- -------

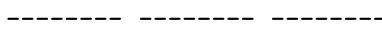

------- ------- -------

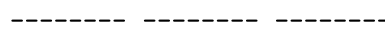

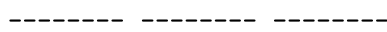



-------- -------- -------

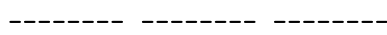

------- ------- ------

40375.000 ---

42875.000 ------- ------ - - -

45375.000

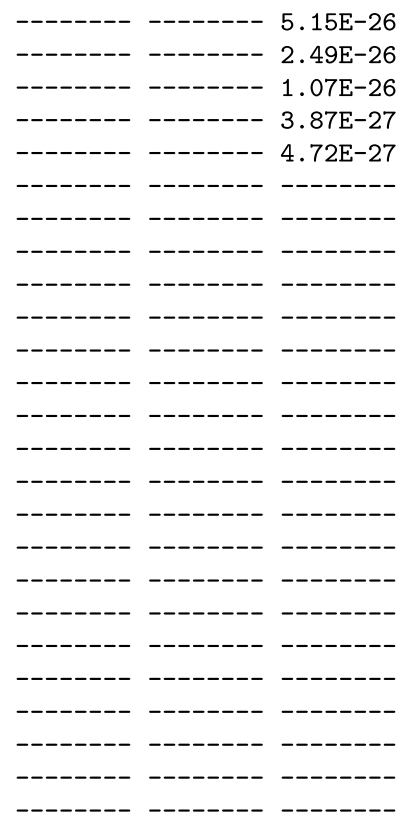


Table 37

The singly differential cross section as a function of the ejected electron energy for double stripping in 5000-25000 keV/u 0^3+, $0^{\wedge} 4+$, $0 ` 5++\mathrm{H} \_2$ collisions.

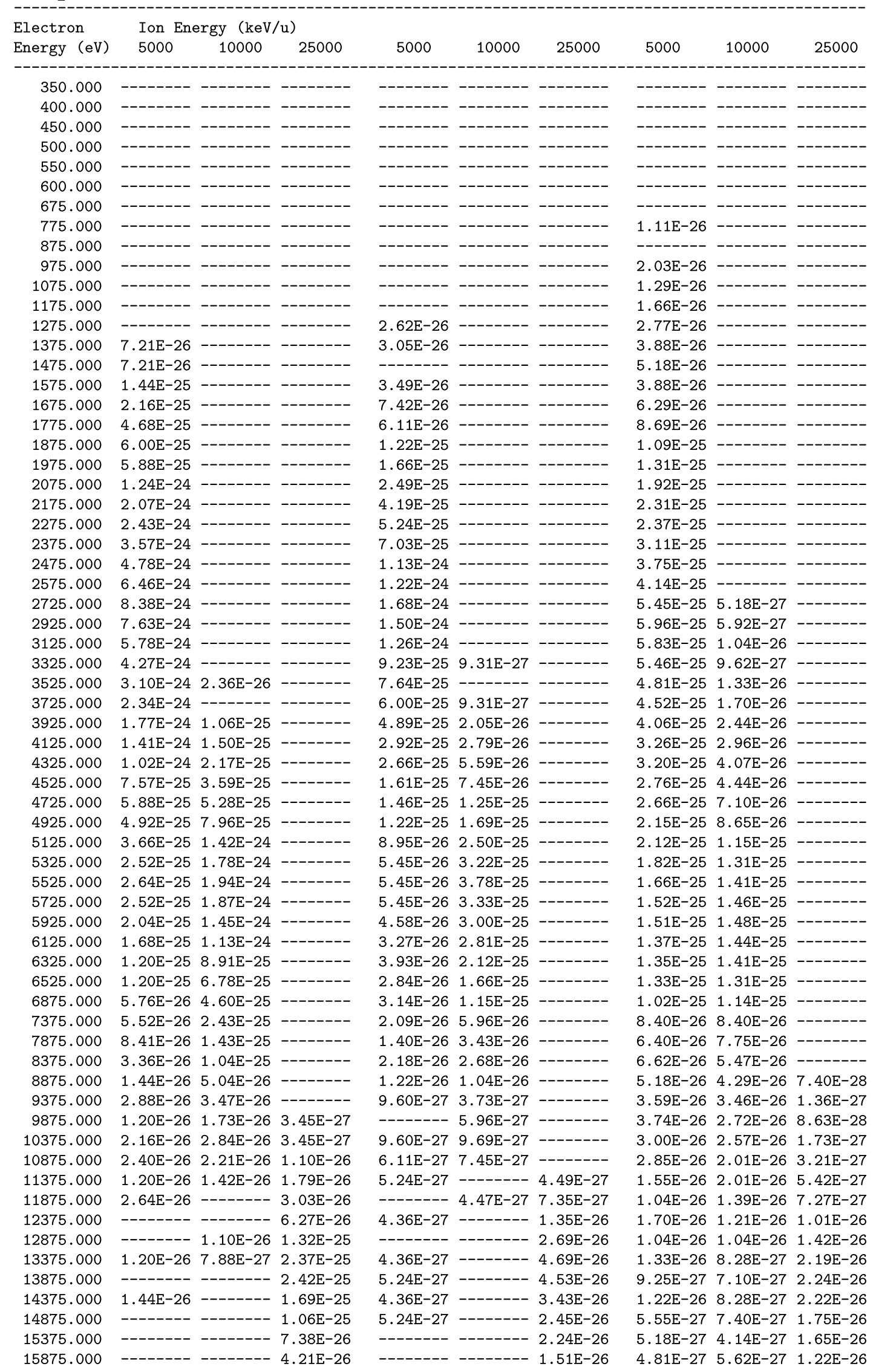



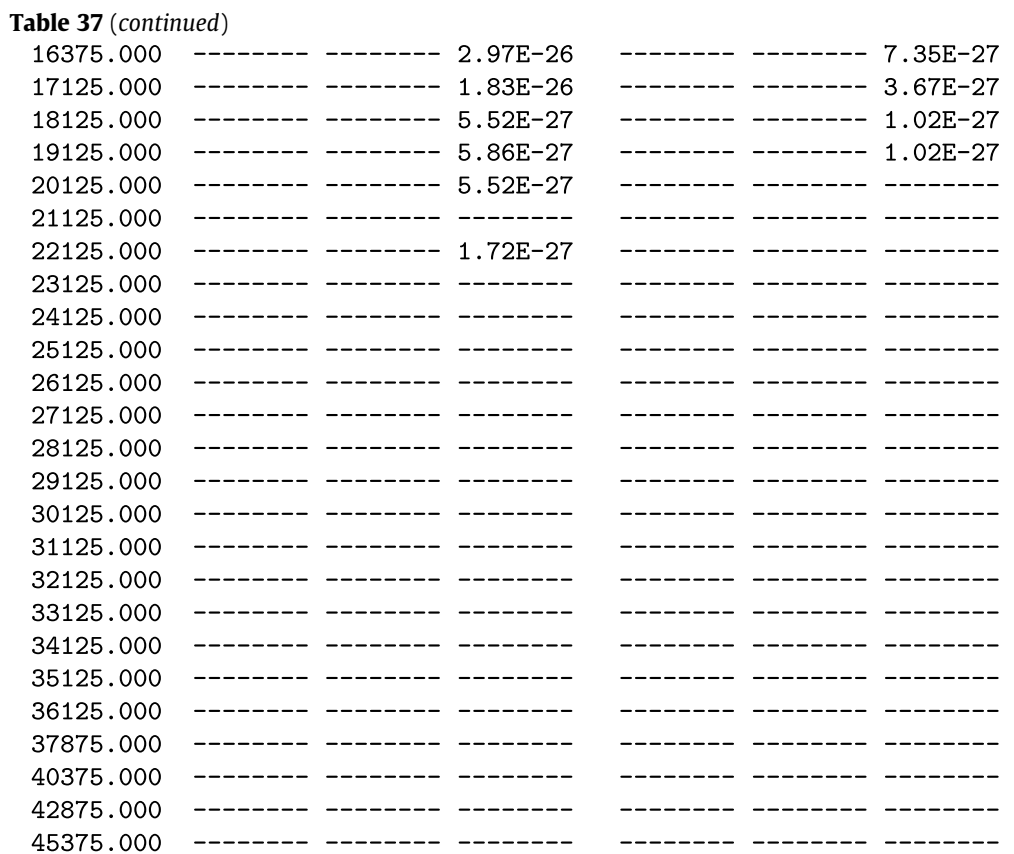
4.07E-27 4.73E-27 $1.20 \mathrm{E}-26$
$\begin{array}{llll}4.07 \mathrm{E}-27 & 4.73 \mathrm{E}-27 & 8.32 \mathrm{E}-27\end{array}$
$\begin{array}{llll}3.33 \mathrm{E}-27 & 4.29 \mathrm{E}-27 & 6.41 \mathrm{E}-27\end{array}$
$2.40 \mathrm{E}-27 \quad 2.66 \mathrm{E}-27 \quad 4.13 \mathrm{E}-27$
$2.77 \mathrm{E}-27 \quad 3.55 \mathrm{E}-27 \quad 4.07 \mathrm{E}-27$
$\begin{array}{lll}1.11 \mathrm{E}-27 & 1.04 \mathrm{E}-27 \quad 2.40 \mathrm{E}-27\end{array}$
$\begin{array}{lll}9.25 \mathrm{E}-28 & 1.33 \mathrm{E}-27 & 2.59 \mathrm{E}-27\end{array}$
------ 2.07E-27 1.42E-27
$\begin{array}{lll}1.11 \mathrm{E}-27 & 1.63 \mathrm{E}-27 & 1.42 \mathrm{E}-27\end{array}$
------ 7.40E-28 1.05E-27
------- $1.18 \mathrm{E}-27 \quad 8.01 \mathrm{E}-28$
------ 7.40E-28 6.78E-28
------ $1.04 \mathrm{E}-27 \quad 6.78 \mathrm{E}-28$
$\begin{array}{lll}------ & 1.04 \mathrm{E}-27 & 3.08 \mathrm{E}-28\end{array}$
----- - -
------- ------- 4.93E-28
------ $8.88 \mathrm{E}-28$ 3.70E-28
------- ------ 5.55E-28
------- ------- 4.93E-28
------- ------- $3.08 \mathrm{E}-28$
-------- -------- --------
-------- -------- --------
------- ------- $1.23 \mathrm{E}-28$
-------- -------- $1.97 \mathrm{E}-28$
------- 2.96E-28

45375.000 
Table 38

The singly differential cross section as a function of the ejected electron energy for double stripping in 5000-25000 keV/u 0^6++ H_2 collisions.

Electron Ion Energy $(\mathrm{keV} / \mathrm{u})$
Energy $(\mathrm{eV}) \quad 5000 \quad 10000 \quad 25000$

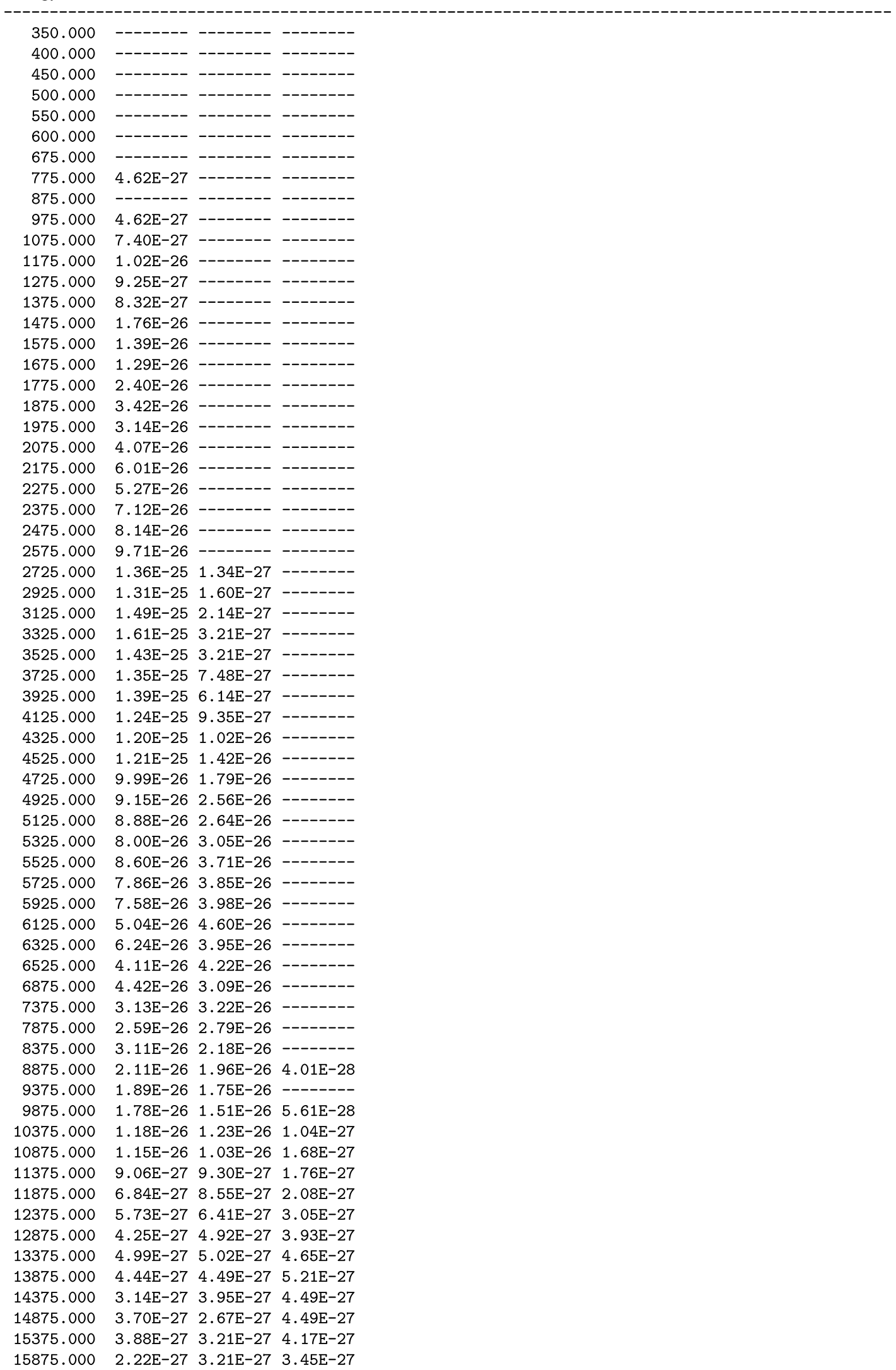


Table 38 (continued)

$16375.000 \quad 3.14 \mathrm{E}-27 \quad 3.10 \mathrm{E}-27 \quad 4.81 \mathrm{E}-27$

$17125.000 \quad 2.59 \mathrm{E}-27 \quad 2.03 \mathrm{E}-27 \quad 3.69 \mathrm{E}-27$

$18125.000 \quad 1.39 \mathrm{E}-27 \quad 1.34 \mathrm{E}-27 \quad 3.21 \mathrm{E}-27$

$19125.000 \quad 1.20 \mathrm{E}-27 \quad 1.34 \mathrm{E}-27 \quad 1.92 \mathrm{E}-27$

$20125.000 \quad 7.40 \mathrm{E}-28 \quad 1.60 \mathrm{E}-27 \quad 2.04 \mathrm{E}-27$

$21125.000 \quad 6.47 \mathrm{E}-28 \quad 9.62 \mathrm{E}-28 \quad 1.40 \mathrm{E}-27$

$22125.000 \quad 6.47 \mathrm{E}-28 \quad 6.95 \mathrm{E}-28 \quad 1.04 \mathrm{E}-27$

23125.000 --.-- 9.08E-28 $8.42 \mathrm{E}-28$

$24125.000 \quad 4.62 \mathrm{E}-28 \quad 6.95 \mathrm{E}-28 \quad 4.81 \mathrm{E}-28$

$25125.000 \quad------5.88 \mathrm{E}-28 \quad 4.01 \mathrm{E}-28$

$26125.000 \quad-----2.67 \mathrm{E}-28 \quad 6.81 \mathrm{E}-28$

27125.000 ------- 3.21E-28 2.81E-28

28125.000 ------ $4.27 \mathrm{E}-28 \quad 3.21 \mathrm{E}-28$

29125.000 ------ $3.74 \mathrm{E}-28 \quad 3.21 \mathrm{E}-28$

30125.000 ------ 2.67E-28 ------

31125.000 -

32125.000 ------ ------ 3.21E-28

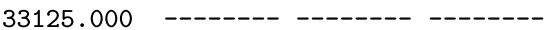

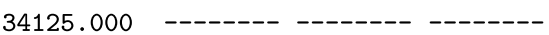

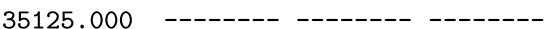

36125.000 -------- -------- --------

37875.000 ----- $1.92 \mathrm{E}-28 \quad 9.62 \mathrm{E}-29$

40375.000 ------ $1.92 \mathrm{E}-28$------

42875.000 ------ ------ ---

45375.000 
Table 39

The singly differential cross section as a function of the ejected electron angle for double stripping in $5000-25000 \mathrm{keV} / \mathrm{u} 0,0^{\wedge}+, 0^{\wedge} 2++$ H_2 collisions.

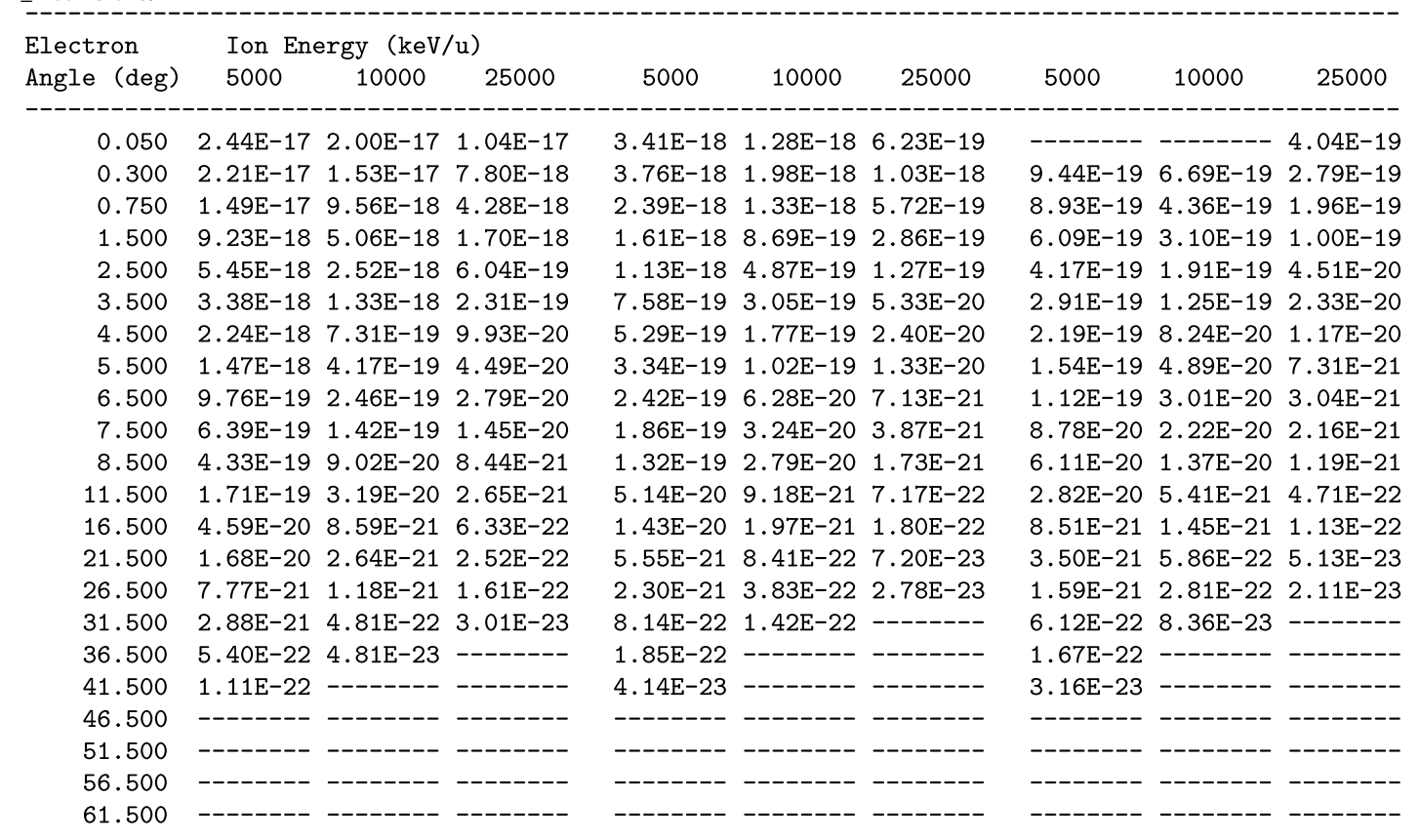

Table 40

The singly differential cross section as a function of the ejected electron angle for double stripping in $5000-25000 \mathrm{keV} / \mathrm{u} 0^{\wedge} 3^{+}, 0^{\wedge} 4^{+}, 0^{\wedge} 5^{+}$ + H_2 collisions.




Table 41

The singly differential cross section as a function of the ejected electron angle for double stripping in 5000-25000 keV/u $0^{\wedge} 6++$ H_2 collisions.

\begin{tabular}{|c|c|c|c|}
\hline \multirow{2}{*}{$\begin{array}{l}\text { Electron } \\
\text { Angle (deg) }\end{array}$} & \multicolumn{3}{|c|}{ Ion Energy $(\mathrm{keV} / \mathrm{u})$} \\
\hline & 5000 & 10000 & 25000 \\
\hline 0.050 & -------- & ------- & -------- \\
\hline 0.300 & $8.05 E-21$ & $3.02 \mathrm{E}-21$ & $1.05 \mathrm{E}-21$ \\
\hline 0.750 & $7.60 \mathrm{E}-21$ & $3.50 E-21$ & 1. $28 \mathrm{E}-21$ \\
\hline 1.500 & $5.80 E-21$ & $2.49 \mathrm{E}-21$ & $8.79 E-22$ \\
\hline 2.500 & 4. $19 \mathrm{E}-21$ & $2.04 \mathrm{E}-21$ & $5.95 E-22$ \\
\hline 3.500 & $4.09 E-21$ & $1.84 \mathrm{E}-21$ & $4.97 \mathrm{E}-22$ \\
\hline 4.500 & $3.54 \mathrm{E}-21$ & 1. $39 \mathrm{E}-21$ & $3.77 \mathrm{E}-22$ \\
\hline 5.500 & $2.96 \mathrm{E}-21$ & $1.18 \mathrm{E}-21$ & $2.94 \mathrm{E}-22$ \\
\hline 6.500 & $2.69 \mathrm{E}-21$ & $1.05 E-21$ & $2.20 \mathrm{E}-22$ \\
\hline 7.500 & $2.31 \mathrm{E}-21$ & $8.06 \mathrm{E}-22$ & $2.04 \mathrm{E}-22$ \\
\hline 8.500 & $1.91 \mathrm{E}-21$ & $7.15 \mathrm{E}-22$ & $1.19 \mathrm{E}-22$ \\
\hline 11.500 & 1. $25 \mathrm{E}-21$ & $4.31 \mathrm{E}-22$ & $7.62 \mathrm{E}-23$ \\
\hline 16.500 & $6.14 \mathrm{E}-22$ & 1.91E-22 & $2.83 E-23$ \\
\hline 21.500 & 3. $20 \mathrm{E}-22$ & $9.86 \mathrm{E}-23$ & $1.36 \mathrm{E}-23$ \\
\hline 26.500 & 1. $92 \mathrm{E}-22$ & $6.01 \mathrm{E}-23$ & $7.04 \mathrm{E}-24$ \\
\hline 31.500 & $8.91 \mathrm{E}-23$ & $2.44 \mathrm{E}-23$ & $3.36 \mathrm{E}-24$ \\
\hline 36.500 & $4.03 E-23$ & $1.05 E-23$ & $7.37 \mathrm{E}-25$ \\
\hline 41.500 & $1.76 \mathrm{E}-23$ & $2.21 \mathrm{E}-24$ & -------- \\
\hline 46.500 & $6.51 \mathrm{E}-24$ & 1. $21 \mathrm{E}-24$ & ------- \\
\hline 51.500 & $2.15 E-24$ & $6.23 E-25$ & ------- \\
\hline 56.500 & $1.82 \mathrm{E}-24$ & -------- & -------- \\
\hline 61.500 & -------- & -------- & -------- \\
\hline
\end{tabular}

